NASA TECHNICAL

TRANSLATION

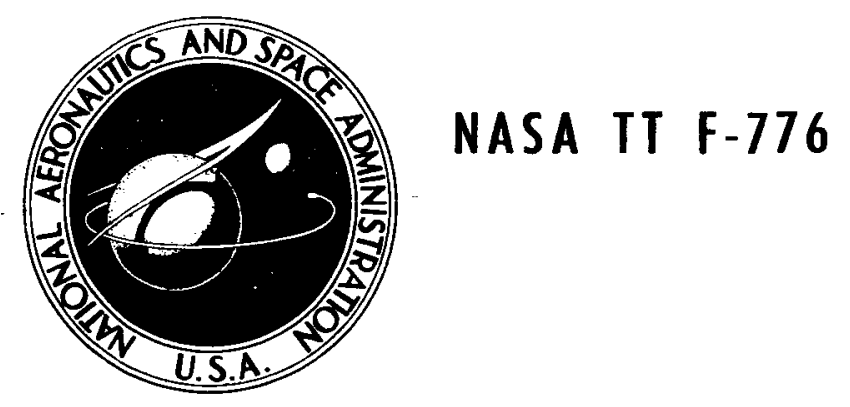

$\stackrel{0}{N}$
$亡$
$上$
$\Sigma$
$\Sigma$

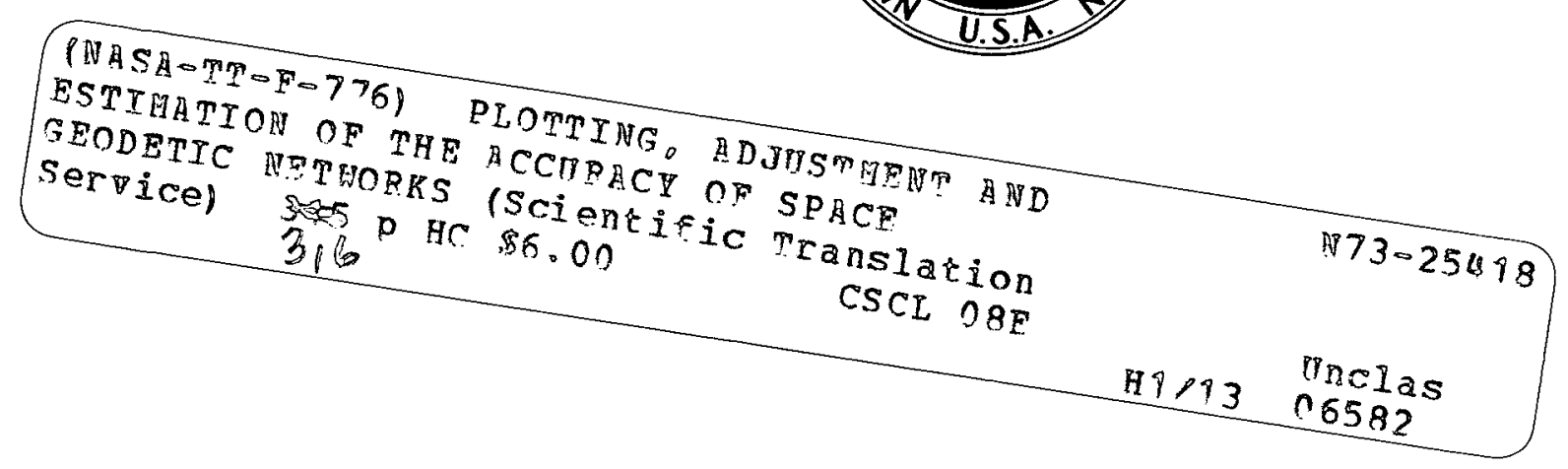

\title{
PLOTTING, ADJUSTMENT AND
}

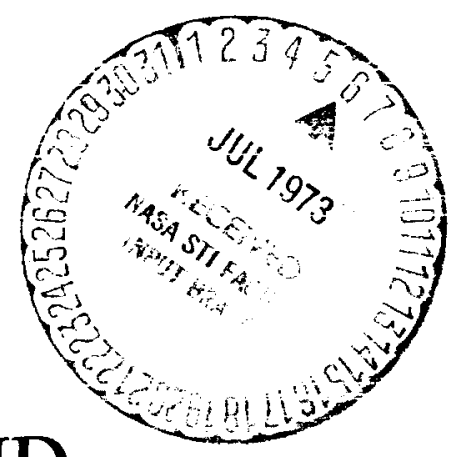

ESTIMATION OF THE ACCURACY

OF SPACE GEODETIC NETWORKS

by Ye. G. Boyko, B. M. Klenitskiy, I. M. Landis,

and G. A. Ustinov

"Nedra" Press, Moscow, 1972

NATIONAL AERONAUTICS AND SPACE ADMINISTRATION • WASHINGTON, D. C. • MAY 1973 


\begin{tabular}{|c|c|c|c|}
\hline 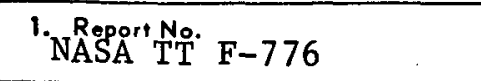 & 2. Government Accession No. & \multicolumn{2}{|c|}{ 3. Recipient's Catalog No. } \\
\hline \multirow{2}{*}{\multicolumn{2}{|c|}{$\begin{array}{l}\text { 4. Title and Subtitle } \\
\text { PLOTTING, ADJUSTMENT AND ESTIMATION OF THE } \\
\text { ACCURACY OF SPACE GEODETIC NETWORKS }\end{array}$}} & \multicolumn{2}{|c|}{ 5. Report Dote May 1973} \\
\hline & & \multicolumn{2}{|c|}{ 6. Performing Organization Code } \\
\hline \multicolumn{2}{|l|}{ 7. Author(s) } & \multicolumn{2}{|c|}{ 8. Parforming Organization Report No. } \\
\hline \multicolumn{2}{|c|}{$\begin{array}{l}\text { Ye. G. Boyko, B. M. Klenitiskiy, I. M. Landis } \\
\text { and G. A. Ustinov }\end{array}$} & \multicolumn{2}{|l|}{ 10. Work Unit No. } \\
\hline \multirow{2}{*}{\multicolumn{2}{|c|}{$\begin{array}{l}\text { 9. Performing Organi zotion Name and Address } \\
\text { SCITRAN, P.O. BOX } 5456 \\
\text { Santa Barbara, California } 93108\end{array}$}} & \multicolumn{2}{|c|}{$\begin{array}{l}\text { 11. Contract or Grant No. } \\
\text { NASw-2483 }\end{array}$} \\
\hline & & \multicolumn{2}{|c|}{$\begin{array}{l}\text { 13. Type of Report and Poriod Covered } \\
\text { Translation }\end{array}$} \\
\hline \multicolumn{2}{|c|}{$\begin{array}{l}\text { 12. Sponsoring Agoncy Nome and Addross } \\
\text { NATIONAL AERONAUTICS AND SPACE ADMINISTRATION } \\
\text { WASHINGTON, D.C. } 20546\end{array}$} & \multicolumn{2}{|c|}{ 14. Sponsoring Agency Code } \\
\hline \multicolumn{4}{|c|}{$\begin{array}{l}\text { 15. Supplementary Notes } \\
\text { Translation of: "Postroyeniye, uravnivaniye } i \text { otsenka tochnosti kosmi- } \\
\text { cheskikh geodezicheskikh setey," Moscow, "Nedra" Press, 1972, pp 1208 }\end{array}$} \\
\hline \multicolumn{4}{|c|}{$\begin{array}{l}\text { 16. Abstroct } \\
\text { The problems of designing, mathematically processing, and determining } \\
\text { the accuracy of three-dimensional geodetic nets compiled from synchronous } \\
\text { observations of artificial earth satellites are examined. The first } \\
\text { part gives brief historical information, and the second part describes } \\
\text { the main methods of space triangulation from photographic, laser, and } \\
\text { doppler measurements. The third part discusses the apriori determination } \\
\text { of the accuracy of elementary figures, series, and continuous nets of } \\
\text { space triangulation. }\end{array}$} \\
\hline 17. Key Words (Selected by Author(s)) & & \multicolumn{2}{|c|}{$\begin{array}{l}\text { 18. Distribution Statement } \\
\text { Unclassified - Unlimited }\end{array}$} \\
\hline $\begin{array}{l}\text { 19. Security Clossif. (of this report) } \\
\text { Unclassified }\end{array}$ & $\begin{array}{l}\text { 20. Security Clossif. (of thi s page) } \\
\text { Unclassified }\end{array}$ & $\begin{array}{l}\text { 21. No. of Pages } \\
315\end{array}$ & $\begin{array}{l}\text { 22. Price }{ }^{*} \\
\$ 6.00\end{array}$ \\
\hline
\end{tabular}

"For sale by the National Technical Information Service, Springfield, Virginia 22151 


\section{ANNOTATION}

The problems are examined of plotting, mathematically processing, and determining the accuracy of three-dimensional geodetic nets compiled from synchronous observations of artificial earth satellites. The first part (Chapters I - III) gives brief historical information, and describes the types of geodetic satellites and equipment used for observations. An examination is made of the coordinate systems and sequence of coordinate transformations for reducing all measured values to one system, the basic moments and the order of preliminary (astrometric) processing of artificial earth satellite photographs, as well as laser and doppler measurements.

The second part (Chapters IV - V) describes the principal methods of space triangulation from photographic, laser, and doppler measurements, and from different combinations. This section is primarily devoted to problems of adjusting space triangulation. The adjusting methods are divided into two groups: the first group includes those in which coordinates of points on the earth and on the satellites are determined concurrently, and the other - only the coordinates of points on the Earth. Since many elements of space triangulation are functions of the measurement results, the generalized principle of least squares is used for their adjustment.

A comparative summary of different methods of adjusting space triangulation is given in conclusion.

The third part (Chapters VI - VII) examines the problems of an apriori determination of the accuracy of elementary figures, 
series, and continuous nets of space triangulation. The optimal figure forms are studied, and some considerations on plotting space triangulation are presented.

The majority of the classifications in the second and third parts have been developed by the authors, and are first presented in systematic form.

The book is designed for scientists and engineers studying space geodesy, and also students in advanced courses and candidates for degrees in geodesy.

There are 6 tables, 80 figures, and 75 references. 
CONTENTS

Page

Annotation

iii

Introduction

CHAPTER 1. DEVEIOPMENT OF METHODS OF GEODETIC UTILIZATION OF ARTIFICIAL EARTH SATELLITES

1. Historical Statement

2. Principal Fundamentals of Methods of Using AES Observations for Geodetic Purposes

3. Satellites Used for Geodetic Observations

4. Equipment for Observation of AES for Geodetic Purposes

CHAPTER 2. COORDINATE SYSTEMS AND THEIR TRANSFORMATION 28

5. Stellar Coordinate Systems 28

6. Transformation of Stellar Coordinate Systems 30

7. Geodetic Coordinate Systems

8. A Common Ground Coordinate System

9. Transformation of Coordinates from One Geodetic System to Another and Conversion to a Common Ground System

10. Time Measurement Systèms

CHAPTER 3. PRELIMINARY PROCESSING OF THE RESULTS OF OBSERVATIONS

11. Problems of Preliminary Processing

12. Preliminary Processing of the Materials of Photographic Observations

13. Preliminary Processing of the Results of

14. Processing of Time Recording Data

CHAPTER 4. SPACE TRIANGULATION PROCEDURE 69

15. Measured and Unknown Values of Space Triangulation 69

16. The Principal Elements of' Space Triangulation

17. Elementary Figures of Space Triangulation, Constructed from Photographic Observations of AES 
Page

18. Elementary Figures of Space Triangulation, Constructed from Combinations of Photographic, Doppler and Laser Observations

19. General Principles and Characteristics of Constructing Space Triangulation

CHAPTER 5. ADJUSTMENT OF SPACE TRIANGULATION 100

20. Types of Conditions Arising in Space Triangulation Networks

21. Adjustment of Space Triangulation by the Parametric Method (the Method of Direct Measurement)

22. Adjustment of Space Triangulation by the Conditional Method with Additional Unknowns

23. Adjustment of Space Triangulation, Formed by Planes by the Parametric Method

24. Adjustment of Space Triangulation Constructed from Chords

25. Information about Adjustment of Space Geodetic Networks Created by the Orbital Method

26. Comparative Survey of Methods of Space Triangulation Adjustment

CHAPTER 6. ERRORS IN THE POSITION OF POINTS IN SPACE TRIANGULATION

27. The Importance and the Problems of A Priori Analysis of the Accuracy of Space Triangulation

28. Methods of A Priori Accuracy Analysis

29. Errors in Space Triangulation Elements

173

30. Estimation of the Accuracy of Elementary Figures

182

198

31. The Effect of Errors in the Position of Starting Points

32. Combining the Formulas for A Priori Estimation of the Accuracy of the Elements and Elementary Figures of Space Triangulation

33. The Effect of the Number of Measurements on the Results of A Priori Estimation of the Accuracy of Space Triangulation Figures

34. A Priori Estimation of the Accuracy of Coordinate Transfer in a Space Triangulation Series

224

35. Errors in the Position of Points in Continuous Space Triangulation Networks 
CHAPTER 7. DATA ON DESIGN OF SPACE TRIANGULATION

36. Fundamentals of Space Triangulation Design

37. General Analysis of the Formulas for A Priori Estimation of the Accuracy of the Elements and Elementary Figures of Space Triangulation

38. Optimum Conditions for the Transfer of Coordinates in a Space Triangulation Series 282

39. Optimum Combinations of Measurements in Continuous Space Triangulation Networks

40. Calculation of the Optimum Characteristics 
PLOTTING, ADJUSTMENT AND ESTIMATION OF THE ACCURACY OF SPACE GEODETIC NETWORKS

Ye. G. Boyko, B. M. Klenitskiy,

I. M. Landis and G. A. Ustinov

\section{Introduction}

The main problems of modern geodesy are determination of the position of points of the Earth's surface in a single coordinate system and the characteristics of the Earth's gravitational field. Solution of these problems within the framework of classical geodesy is characterized by the use of two three-dimensional coordinate systems to detefmine the position of the same point.

The position of a point is calculated on an ellipsoid, and the helght of this same point - with respect to a quasi-geoid. This duality is aggravated even more by the fact that every country (or group of countries) has its own reference ellipsold, 1.e., It establishes a special coordinate system even to calculate a systematic system of points. This is how the large number of national coordinate systems developed and, as a result, the problem of establishing relationships between them.

Up to the second half of the 1950's, the main source of information to solve geodetic problems was the results of observations carried out on the Earth's surface, and of the sighting targets located on it. This naturally limited the possibilities of studying the Earth's shape by geometric methods primarily due

* Numbers in the margin indicate pagination in the original foreign text. 
to the relatively small lengths of the sides, providing visibility of the sighting target. Because of this, it was impossible to relate the continuous triangulation net of a territory, divided by large water expanses, to obtain sufficiently large arcs of degree measurements and to create a single coordinate system for the entire Earth.

Solution of this problem is possible in principle on the basis of using gravimetric data. As a result, local systems will be reduced to a single system with the origin at the center of the Earth's mass. However, a detailed study of the Earth's gravitational field entalls a number of difficulties and, primarily, the necessity of measuring gravity at sea. In connection with this, the problem obtains only an approximate solution.

A complex solution of basic geodetic problems would be possible by observations of space objects, moving in the Earth's gravitational field. Such objects, being high-altitude sighting targets, would expand the possibilities of the geometric method considerably. Study of the motion of these objects in the Earth's gravitational field would lead to a determination of its characteristics.

Attempts to use the natural satellite of the Earth - the Moon - as such an object led to the development of a new branch of science - space (satellite) geodesy. However, observations of the Moon as an object for space geodesy had no important practical significance.

The appearance of artificial Earth satellites (AES) contributed to the development of space geodesy and led to important results within short periods both in determination of the location of points in a unified global system and in a study of the Earth's gravitational field. 
Two main trends of space geodesy have now quite clearly formed. The first trend includes a group of methods for the joint determination of the Earth's geophysical parameters and the coordinates of points. This section is often called the dynamic method. A vast literature is devoted to a description of this method. It is more completely and thoroughly outlined in the investigation of W. Kaula "Satellite Geodesy," written in 1966 and published in 1970 in the Russian language by the "Mir" Publishing House.

Construction of three-dimensional geodetic nets with the aid of synchronous observations of AES comprises the second trend, which has been called the geometric method, or space triangulation. This method cannot be considered as a simple improvement of known methods of classical geodesy, leading only to an increase in the length, of the sides of geodetic nets. A number of characteristics of measuring information and its mathematical treatment generated a large number of new problems, not characteristic of the methods of ordinary. geodesy.

In connection with this, numerous investigations have appeared both in domestic and in the foreign literature, devoted to problems of applying different systems of coordinates, to a consideration of optimum plotting of space triangulation and its preliminary processing and adjustment.

An attempt has been made in the present investigation to systematically outline the problems of a priori evaluation of the accuracy, projection and mathematical treatment of measurements in space geodetic nets, created by synchronous observations of AES. Taking into account the great importance of these problems for solution of scientific and technical problems, the authors attempted, along with the necessary theoretical justification, to reduce the final results to a form convenient for practical use. 
In conclusion, we note that space geodesy, like any new branch of science, is developing very rapidly, and its individual disclplines and the relationships between them have not yet been settled and have not yet passed the necessary test of time. The authors well understand that there are many sections and problems in the book, exposition of which could be improved; therefore, they will gratefully accept all critical comments of readers. 


\section{CHAPTER I}

DEVELOPMENT OF METHODS OF GEODETIC UTILIZATION

OF ARTIFICIAL EARTH SATELIITES

1. Historical Statement

Use of the Moon

Long before the appearance of artificlal Earth satellites (AES), the possibilities of geodetic utilization of the observations of space objects, having a discernible diurnal parallax, were evaluated for their merit. Prior to the launch of the first AES, the only such method was the Earth's natural satellite - the Moon. Scientists attempted to use observations of it for geodetic purposes for about 200 years.

The diurnal parallax of the Moon does not exceed 6I'32". Consequently, the maximum parallactic shift of the Moon with respect to the stars, even when it is observed on the horizon at points opposite the Earth's surface, will comprise a little more than $2^{\circ}$. As a result, when purely geometric methods of transmission of coordinates between points of the Earth's surface using observations of the Moon are utilized, satisfactory geometric plots cannot be obtained.

It is easy to calculate that, even if the geocentric motion of the Moon is known with high accuracy, and the bearings toward the center of the Moon are measured with an error of $0.02 "$, the position of a point on the Earth cannot be calculated more precisely than $100 \mathrm{~m}$. 
Therefore, it is quite natural that, in turning to the problem of geodetic utilization of the Moon, scientists were faced primarily with the problem of developing those methods and equipment for observation which would provide the highest accuracy. The problem of geodetic treatment of observations has moved aside to a secondary position.

Phenomena of solar eclipses and osculations of stars by the Moon are employed in visual observations of the Moon. The essence of observations in this case reduced to fixation of the moments of contact of the edges of the visible disks of the Moon and sun or moments of osculation of the stars by the Moon. The measurement accuracy is affected by a large number of factors, primarily by the unevenness of the visible edge of the Moon.

Special maps of lunar profiles have been created to take into account corrections for "lunar topography." Such maps, for example, were created by Hain in 1914 and by the Tartu Observatory in 1952. The accuracy with which the position of an individual point of the lunar limit is calculated by these maps is apparently no greater than \pm 0.1 " [5], which naturally does not satisfy the requirements of geodetic measurement. Lunar profile maps, created at the Observatory imeni Engel'gardt in Kazan' in 1958 [39] and at the Naval Observatory in Washington in 1963, are possibly somewhat more accurate. However, a considerable increase in accuracy may be expected only from a direct study of the Moon by photography of its surface from an artificial lunar satellite and by making direct geodetic measurements both between points of the Earth, Moon and spacecraft, and on the lunar surface. 
Besides the creation of lunar profile maps, special methods and equipment for recording the moments of observation have been proposed which raise the accuracy of results. These are, for example, the method of observation at equal position angles [63] and photoelectric recording of osculations [75].

Methods of obtaining topocentric directions toward the Moon by direct photography of it on a background of the stellar sky are well known. Attempts to obtain photographic images of celestial objects were undertaken soon after discovery of the photographic process.

It is interesting to note that the first celestial object recorded on photographs was the Moon [6]. The first good photograph of the Moon was obtained by Draper in 1840. During the period 1911 - 1917, King obtained a bearing toward the Moon with an error of $\mathrm{m}_{\mathrm{a}}= \pm 1 "$. During the period 1920 - 1928, Boehm, using Hain's photographs, raised the accuracy of determining the bearing toward the Moon to $\pm 0.5-0.7 "$. The main difficulty in obtaining good images of the stars and Moon on a single negative occurred due to the comparatively high speed of motion of the Moon with respect to the stars and its great brightness. These difficulties were overcome as a result of creating special lunar cameras $[5,38]$, in which the motion of the Moon and attenuation of its brightness (a dark plane-parallel Markowitz plate) were compensated for, and exposures of different length (Mikhaylov's "shutter") were made for the Moon and stars. Observations made by Markowitz during the International Geophysical Year (IGY) determined the central position of the visible disk of the Moon with respect to reference stars on a photographic plate with an accuracy of $\pm 0.15^{\prime \prime}$. Markowitz concludes from this that multiple photographic observations of the Moon determine the geocentric 
position of the observation point with an accuracy of $\pm 30-40 \mathrm{~m}$ [61]. Apparently, this is the limit of accuracy, the approximation of which is theoretically possible by photographic observations of the Moon.

Despite the great success in Improving methods of geodetic use of the Moon, creation of special equipment and methods of observation, these investigations did not go beyond the limits of individual experiments. A good lllustration of this is two predictions. The first was made in 1768 by Johann Albert Euler, the son of the famous Leonard Euler. Considering the possibility 17 of deriving a meridian ellipse from observations of the Moon from a number of points located on a single meridian, he wrote: ". . If the Moon were closer to the Earth or there were other bodies close to the Earth which could be observed from different points of a single meridian, this method of determining the shape of the Earth would be simpler and more convenient than a method based on degree measurements in triangles" (Snellius's triangulation method). The second proposition belongs to the renowned Soviet scientist, academician A. A. Mikhaylov, and was made in 1957 literally on the eve of the launch of the first artificial satellite in the USSR: "The distance of the Moon from the Earth and the smaliness of the lunar parallax caused by this, make geodetic use of the Moon a difficult if not a thankless task." And further: "Perhaps, in the future the Moon will be replaced by an artificial satellite, moving near the Earth, the observation of which will solve geodetic problems more accurately" [38].

Väisäla's Method

In 1945, the Finnish sclentist Väisäla [70] proposed calculating the direction of a chord, connecting two ground points, by simultaneous observation of flares fired from alrcraft or balloons. In this case, unlike the previous triangulation with 
high-altitude moving sighting targets, it was proposed to photograph the flare on the background of the stellar sky rather than to use goniometric instruments to measure the bearings toward the flare. Then the direction toward the flare could be calculated by the camera method after photographic measurement of the position of the flare with respect to stars with known coordinates.

Experimental calculations of the direction of the chord between two points (at Helsinki and near Turku) were carried out in 1946. Magnesium flares fired from a balloon were photographed. This first experiment may be considered the beginning of threedimensional astral triangulation, but this method has not found extensive use, because the altitudes to which the sighting targets were raised were not adequate for increasing the length of the triangulation sides.

\section{Space Geodesy}

Only after the launch of the first artificial Earth satellite in the USSR on 4 October 1957 did the real possibility of constructing three-dimensional triangulation with large sides appear. The first practical experiments carried out both in the USSR and abroad [53, 72] indicated the great promise and possibility of high accuracy of the new method.

Construction of the first experimental network of AFS observation points for geodetic purposes was begun by the Smithsonian Astrophysical Observatory (United States) in 1959. It included 12 points equipped with equipment to photograph AES on the background of the stellar sky. Investigations on the observation of AES for geodetic purposes have been conducted in the USSR and the socialist countries since 1961 upon the 
Initiative of scientists of Pulkova Observatory and of the

Astronomical Council of the Academy of Sclences of the USSR. Photographic observations of different AES have already yielded extensive material which leads to the construction of a geodetic net, encompassing a considerable area [53]. These investigations are being continued successfully even now in a program of international cooperation.

W1th the development of space geodetic nets, photographic observations have begun to be supplemented by laser and radiotechnical measurements, independently of weather conditions, which yield the distances, radial components of AES speed and differences of the distances from the observation point to the AES positions with high accuracy.

\section{Principal Fundamentals of Methods of Using AES} Observations for Geodetic Purposes

Three vectors (Figure I) are connected by the relation

$$
\bar{r}_{K}=\bar{r}_{C}+\bar{\rho}_{C K}
$$

Essentially, Equation (2.1) is the basic equation of space geodesy [15].

If point $\mathrm{C}$ corresponds to the AES observation point and point $K$ corresponds to the position of the satelite, radius vector $\bar{r}_{C}$ determines the position of the observation point, radius vector $\bar{r}_{K}$ determines the position of the satellite, and vector $\bar{\rho}_{\mathrm{CK}}$ determines the position of the satellite with respect to the observation point (the topocentric position of the satellite). 


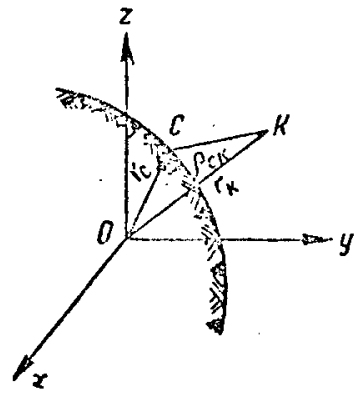

Figure 1.

As we know, every fixed vector in space is determined by the point of application, by the direction and by the modulus. Depending on which, values determining the three vectors of Equation (2.1) are known, the following problems may be distinguished.

$K$. In this case, the position of the observation point is assumed to be known, 1.e., vector $\bar{r}_{C}$ has been calculated. Vector $\bar{\rho}_{C K}$ must be measured. It is possible in this case that if all three of its components have been measured, the problem is solved directly by a single Equation (2.1). If only the angles characterizing the direction of vector $\bar{\rho}_{C K}$ have been measured, yet another Equation (2.1), compiled for another observation point, is required for the solution. If only the distances to the satellite (the modulus of vectors $\bar{\rho}_{\mathrm{CK}}$ ) are measured, it becomes necessary to make observations from three points to obtain three equations of (2.1). When measuring the difference of distances according to two posi- 19 tions of the satellite, observation from six points is necessary.

\section{The inverse problem is calculation of vector $\bar{r}_{C}$, i.e.,} the position of observation point $C$. We shall assume that vector $\bar{r}_{\mathrm{K}}$ has been calculated - the position of the satellite is known. The vector $\bar{p}_{\mathrm{CK}}$ is measured. When all three of its components have been measured, the problem is solved by a single Equation (2.1). When measuring only the angles characterizing the direction of vector $\bar{p}_{\mathrm{CK}}$, it is necessary to observe two positions of the satellite to compile two Equations (2.I). When measuring only 
distances to the satellite, three observations are required to compile three equations of observation. When calculating the position of a point by the measured differences of distances, three pairs of AES positions are required.

In connection with the fact that the direction and value of vector $\bar{r}_{K}$ varies in time - the satellite moves - the necessary element of all constructions is time $S$. In individual cases, time may emerge as the measured value, and sometimes it must be calculated along with other unknowns.

To construct geodetic nets from AES observations, joint solution of many direct and inverse problems is required. Essentially, two methods are possible.

1. Combination of the direct and inverse problems by calculation of the variation of vector $\bar{r}_{K}$ in time, i.e., the orbital method. In this case the direct problem is used to calculate the satellite orbits $-\bar{r}_{K}(S)$, and the inverse problem

- to calculate the position of observation points (although mathematical treatment of all measurements is carried out jointly). It is natural that time displacement of observations, made for solution of direct and inverse problems (Figure 2a) is not required in this case.

2. Calculation of individual (instantaneous) values of vector $\bar{r}_{K}$. In this case observations should be made simultaneously (synchronously) from the calculated and initial points, i.e., from a point which is taken either as the initial or may be calculated by other positions of the satellite. Such observations, in particular, may be photographic, Doppler and laser (Figure 2b). 


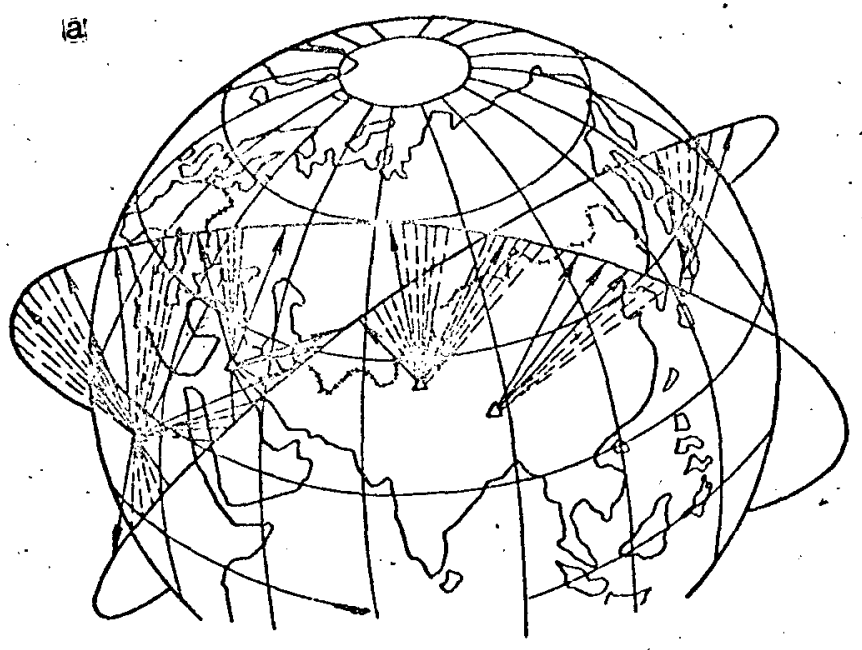

b

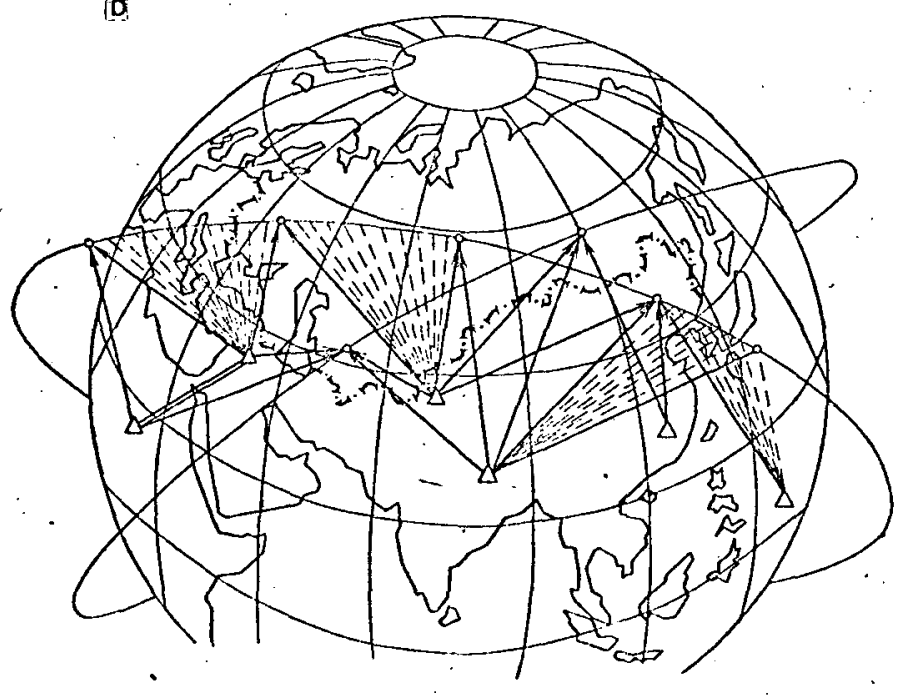

The first method, in which the intermediate elements for transmission of observation point coordinates are the orbital parameters, are called the orbital elements. The second method, based on synchronous observations, has been named space triangulation.*

Both methods (the orbital and triangulation) solve the same problem calculation of the point coordinates. However, the methods of solution differ considerably.

In space triangulation all constructions are based on geometric functions; therefore, space triangulation is a purely geometric method.

Figure 2.

"Sometimes the term "astral triangulation," meaning that the directions to the AES are calculated with respect to the stars, or the term "satellite triangulation" are used in the literature. In our view, these terms are just as justified (or unfustified) as the term "space triangulation." Moreover, it should be noted that the angular values may be measured not only in a stellar coordinate system, but linear measurements in general are invariant with respect to coordinate transformations. 
In the orbital method laws of satellite motion are used which are based on an accurate knowledge of all effective forces. The dynamic method of processing the results of AES observations is used to obtain these data. The ultimate purpose of this method is the problem of calculating the Earth's gravitational field and shape. The essence of the method is that the precalculated motion of the satellite is compared to the observed motion, and the characteristics of the Earth's gravitational field and shape are refined with respect to the perturbations. Circumterrestrial artificial satellites are much more convenient for these purposes (in any case more convenient than the Moon), since their motion is almost completely determined by the Earth's gravity field. In connection with the fact that calculation of the density of the upper layers of the atmosphere and the effect of a number of space factors have not yet been adequately studied, very extensive observational material must be included in the processing to reduce their effect.

Until adequately extensive, accurate and well distributed measurements are obtained and carefully processed, the basic problem of the dynamic method cannot be solved and, consequently, precise laws of AES motion cannot be obtained.

Therefore, at present the use of the orbital method to calculate the point coordinates with a high degree of accuracy is limited. Space triangulation does not depend on the theory of AES motion and, therefore, its accuracy is determined only by the merits of geometric construction and by measurement errors. This also explains the overwhelming use of the space triangulation method to calculate point coordinates. 
It should be noted that there is no absolute boundary between the methods of processing the results, since, for example, both the dynamic and orbital methods are connected by a common theory of satelite motion.

Observation methods may be identified with those of processing the results, since the results of optical or radiotechnical observations may be used to solve both dynamic and geometric problems. In turn, synchronous observations may be used for both orbital and triangulation methods.

\section{Satellites Used for Geodetic Observations}

The process of AES observation reduces to fixation of certain signals coming from it. If on-board devices, emitting or relaying signals sent from the Earth, are used for geodetic purposes in observation of $\mathrm{AES}$, such $\mathrm{AES}$ are called active. If the satellite is observed only in reflected solar light, it is called passive.

Usually, passive AES are specially not designated for geodetic measurements. Therefore, if they are equipped with on-board equipment, it is not used for geodetic purposes. In the opposite case, the satellite should be related to active satellites.

American communication satelites - balloons - should be primarily included among the satelites of no special geodetic designation. The first of these, Echo-1, was launched in August of 1960. It was an inflated sphere, made of a special film, which was filled after insertion of the satellite into orbit. A second similar satellite, Echo-2, was launched in January 1964. 
Satellites of the Echo class, having a rather large diameter (up to $40 \mathrm{~m}$ ), are easily visible on the background of the: stellar sky and are accessible for observations by simple optical means.

Observations of these satellites laid the basis for creation of space triangulation nets. Observations of Echo-1 and Echo-2 for geodetic purposes permitted a primary space triangulation net to be constructed, which encompasses a number of points on the territory of the USSR and the socialist countries [53]. The Echo-1 and Echo-2 satellites have now ceased to exist. The successes achieved with geodetic use of these satellites have led to creation of a special geodetic passive AES - Pageos. This balloon satellite, launched in June of 1966, has a diameter of about $30 \mathrm{~m}$, and its outer surface is covered with a thin layer of aluminum, which ensures a high reflectivity. The orbital altitude of the Pageos satellite (at the moment of launch $4600 \mathrm{~km}$ above the Earth's surface) permits simultaneous photography of it from points located at distances up to $5000-6000 \mathrm{~km}$ from each other.

Successful use of passive Echo AES for geodetic purposes brought to light the problem of creating special active geodetic satellites, corresponding to the characteristics for solution of geodetic problems. The requirements for geodetic satellites were formulated in May of 1964 after the Sixth Session of the International Committee on Space Research.: These requirements reduce to provision of minimum effects of atmospheric drag: and their variation, best optical visibility and to selection of optimum orbital parameters. The general requirements for satellite orbits, intended to solve geometric problems, are the following: orbital altitude should be, on the one hand, sufficiently low to increase the accuracy of calculating point coordinates, and, on 
the other hand, should be sufficiently high to ensure visibility from points remote from each other. The orbital inclination should be sufficiently. high that the satellite may be observed at high latitudes, and eccentricity selected such that the required variation of altitudes is provided for observation from points located at different distances.

For the successful solution of geodetic problems, it is desirable to have the following on-board equipment on the satellite:

- an optical beacon emitting momentary powerful light flashes. Generation of light flashes should be accomplished by a previously designated program, providing the best geometric construction of the net. The program may be executed by single commands from Earth or by signals of special on-board devices;

- radio transmitters of highly stable frequency to produce Doppler variations, operating in no less than two bands;

- relays for radio signals transmitted from Earth for measurement of distances;

- angle reflectors to make laser measurements;

- highly accurate clocks and data storage to perform programmed activation of the on-board equipment. Moreover, time checks may be accomplished by the signals of on-board time transmitted to the observation points;

- a radio altimeter. 
Among the active satellites, the American Geos (Geodetic Earth Orbiting Satellite) should be noted. The satellites are equipped with an optical beacon, which emits a series of seven flashes each with intervals of four seconds between flashes. Along with the optical beacon, there are Doppler transmitters, angle laser reflectors and a system for simultaneous determination of distance and radial velocity on the satellite [62]. Atomic clocks, which emit signals for measurements at the given programmed moments with a high degree of accuracy (up to:50 microns/second), are installed on the satellite.

The French geodetic satelites the "Diadem;" launched in February 1967, play an important role in the creation of a worldwide geodetic net. These are two similar satellites, equipped with a system of Doppler transmitters and laser reflectors.

The number of active and passive AES, used for geodetic purposes, is increasing from year to year. Interesting designs of special geodetic satellites and systems have been proposed. One of such design'envisions the creation of a satellite whose motion is not affected by the atmosphere. Elimination of the effect of the atmosphere opens up extensive possibilities in a study of the Earth's gravitational field from observations of low-orbiting AES. This is achieved by inclusion of a heavy satellite into a lightweight correcting shell.

The design of the Geos-C program, which envisions joint use of several AES in different orbits, is of great interest. 
Among the requirements placed on equipment designed for observation of AES are operativeness, reliability, and accuracy of measurements. Depending on the problem being solved, one of these requirements becomes decisive. High accuracy of measurements with comparatively small dimensions and weight of the equipment are primarily required for geodetic purposes. Therefore, numerous optical (aerological theodolites, phototheodolites, AT, TZK*, etc.) and radiotechnical goniometric equipment is not used for geodetic purposes because of its low accuracy (bearings are measured with an accuracy up to several minutes). Among goniometric equipment, the highest accuracy is provided by photographic units, which permit a satelilte to be photographed against the background of stars.

Both radiotechnical and laser systems are employed to measure distances to AES. However, laser systems provide higher accuracy of measurements. Moreover, Doppler systems, which measure variation of AES distance at a fixed time interval (or radial velocity component), are widely used for observations of AES.

Photographic devices, intended for observations of AES for geodetic purposes, despite their variety, may be divided into three large groups: fixed (azimuthal and ballistic), star trackers (star and equatorial) and satellite trackers.

The simplest in working principle are photographic devices of the first group. In these devices the camera is rigidly secured with respect to the horizontal coordinate system at the moment of observation. Images of stars and AES are obtained in

*[Translator's Note: Expansion unknown.] 


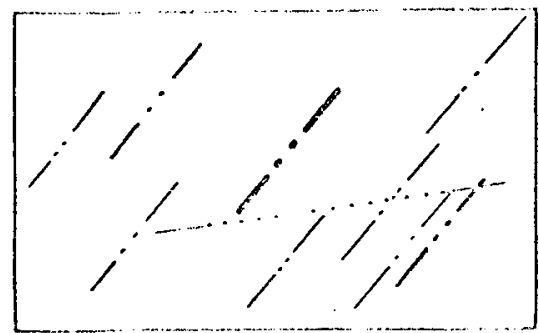

Figure 3.

the form of hatchings and dots on the photographic plate (Figure 3). Only point images of stars and AES are used for astrometric processing. However, due to the different velocity and brightness of stars and AES, it is impossible to receive their point images simultaneously. The required length of satellite exposures is from several hundredths to tenths of a fraction of a second, and that of star exposures - several seconds. Therefore, a corresponding number of satellite exposures is made at an interval of $10-20^{\mathrm{s}}$, and before and after - 2 - 3 star exposures. The moments of opening and closing of the shutter during each exposure are recorded to calculate the time of receipt of the point images of the stars and AES.

The first mass photographic observations of AES for geodetic /15 purposes were carried out with fixed devices. They soon became extensively used due to the simplicity of design, small size, and convenience in operation. Usually, the first cameras of this group were produced on the basis of available aerial photographic equipment.

The first unit of this group in the Soviet Union was the UFISZ-25, created in 1959 on the basis of the aerial photography apparatus the NAFA-25/3S [36]. The focal distance of this camera is $25 \mathrm{~cm}$, aperture ratio is $1: 2.5$, the objective is an Uran-9, the shutter is a louver type and the weight is about $30 \mathrm{~kg}$. The opening and closing time of the shutter is recorded by a digital chronograph, operating from a quartz generator. The camera permits passive satelites with a brighness up to $4^{\mathrm{m}}$ to be photographed at an angular velocity up to $1 \% /$ second. The bearing to the AES is calculated with an error of $\pm 5-7 "$. 
The fixed photographic units Wild-WC-4 and the RC-1000 have been widely used abroad.

The Wild-WC-4 unit has been produced since 1962. It is designed on the basis of the aerial photography camera $\mathrm{RC}-5$ and is mounted on a modified support of the Wild-T-4 astronomical universal theodolite. The focal distance of the camera is $305 \mathrm{~mm}$; the objective is an "Astrotar" and the aperture ratio is $1: 2.6$. The Wild-WC- 4 unit permits both active and passive satelites with a brightness up to $7^{\mathrm{m}}$ to be photographed. The accuracy of determining direction to the AES is about $\pm 2 "$.

The RC-1000 photographic unit was developed in 1961 with the main purpose of photographing the flashes of the optical beacon of an active geodesic AES. The focal distance of the camera is $1000 \mathrm{~mm}$. The objective is a telescopic "Telephoto" type, and the aperture ratio is $1: 5.0$. The camera provides images of flashes no weaker than $8^{\mathrm{m}}$. The direction to the AES is determined with an error of $\pm 1-2$ ".

Photography is accomplished in the Wild-WC-4 and RC-1000 units on glass plates, unlike the UFISZ-25 unit, in which film is used.

The photographic unit of the second group maintains a fixed bearing of the camera's optical axis in the stellar coordinate system during observations. Because of this, the star images on the plate (film) do not shift during photographing and all stars are received in the form of points. The type of photograph obtained during observation of a passive AES by a startracking camera is shown in Figure 4. The process of photographing passive AES on these cameras differs from that of photography by fixed cameras in that it is unnecessary to 


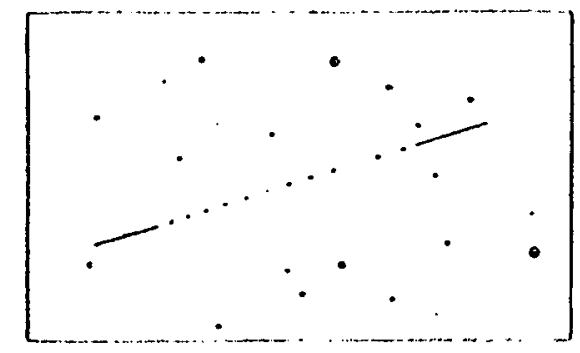

Figure 4.

produce special star exposures. The process of photographing the flashes of active AES is reduced to opening of the shutter until the moment of transmission of the first flash in the series and closing it after transmission of the last flash.

One of the most accurate devices of this group is the FAS-3A, created in the USSR in 1969 [36]. This unit is similar to the above RC-1000 in accuracy. The camera of this unit has a mirror- 116 lens objective a focal distance of $480 \mathrm{~mm}$ and an aperture ratio of $1: 1.9$. Photography is accomplished on glass plates. Unlike the forelgn cameras of this group, tracking of the stellar sky in the FAS-3A is accomplished during photography with the aid of an original device, developed by K. Lapushnaya and M. Abele [36] in 1965 for the AFU-75 unit. This device has been named an equatorial platform.

Cameras of the third group are more universal and photograph satellites over a wide range of brightnesses and speeds. The distinctive feature of these cameras is the possibility of tracking AES. Tracking is accomplished either by shifting the plate (film) to compensate for the shift of the AES image or by the camera tracking the satellite. As a result, the satellite image is maintained for a long period on one point of the plate ( $f 1 \mathrm{~lm})$, and at the same time an increase in the length of satellite exposure is achieved. The most typical representatives of this group are the domestic AFU-75 (Figure 5) and the VAU, the American Baker-Nunn (Figure 6 ) and the camera built by the Zeiss Company - the SBG (East Germany). 


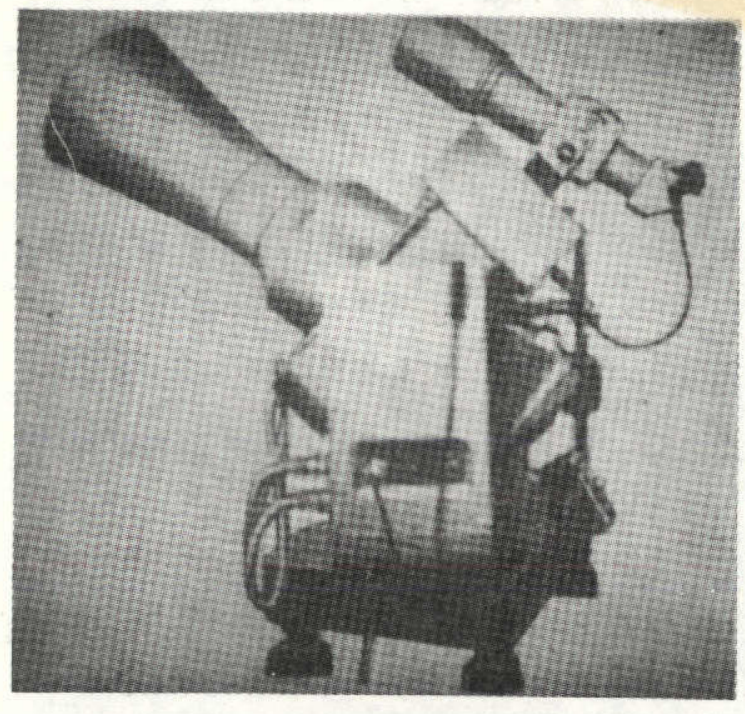

Figure 5.

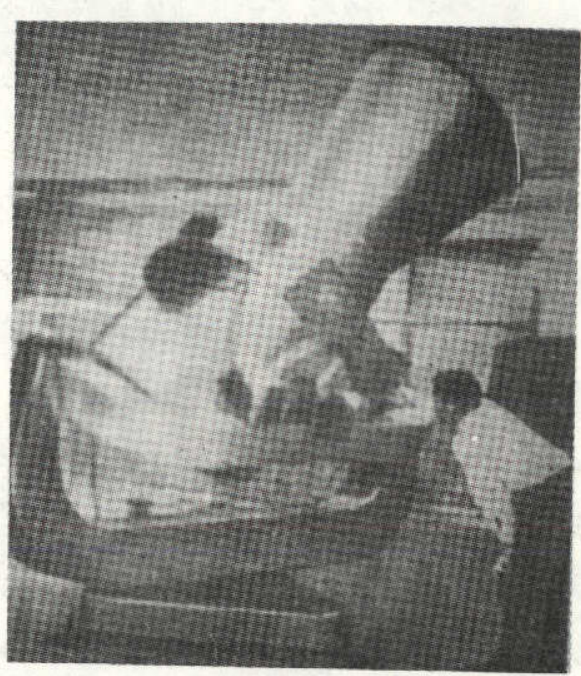

Figure 6.

The AFU-75 camera was designed in 1965. This camera has an equatorial platform for tracking stars. Tracking of AES is accomplished by shifting a clamped plate with a film in a cassette. The focal distance of the camera is $735 \mathrm{~mm}$ and the aperture ratio is $1: 3 \cdot 5$. The camera permits satellites with a brightness up to the ninth stellar magnitude to be photographed in the tracking mode. The accuracy of determining the direction to the AES is $\pm 2-3^{\prime \prime}$. The camera weighs about $350 \mathrm{~kg}$.

The VAU camera (Figure 7) began operation at the Zvenigorod Station of the Astronomical Council of the USSR Academy of Sciences in 1969. This is a universal camera which permits not only AES, but distant space objects to be photographed. Operational control of the camera is automated to the maximum extent. The camera has a highly accurate timing device. A more detailed description of this camera is presented in [36]. 


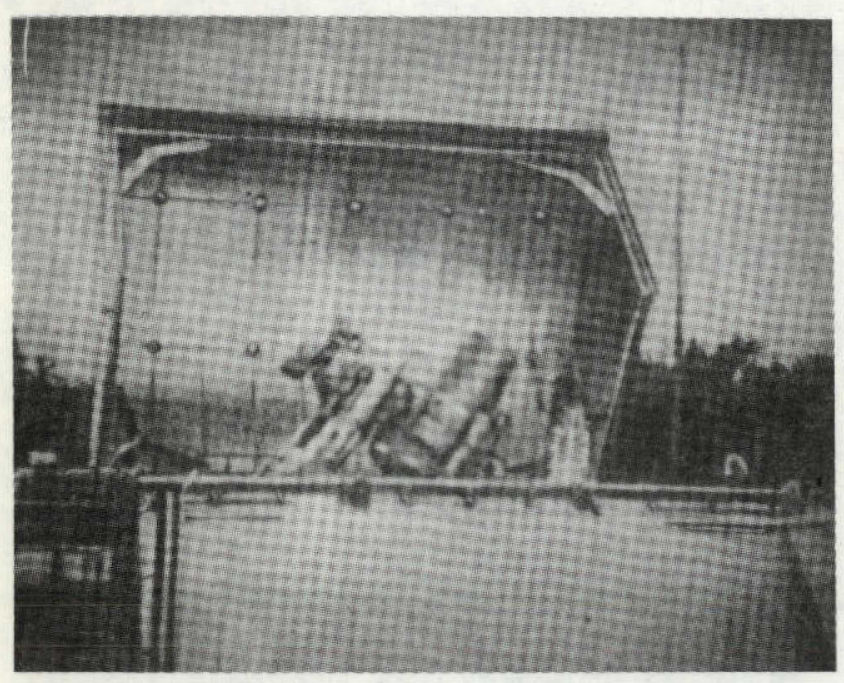

Figure 7.

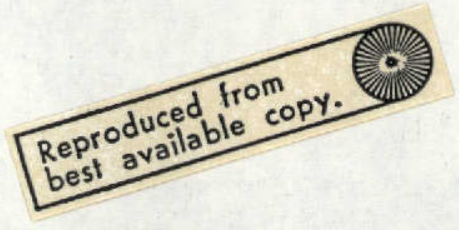

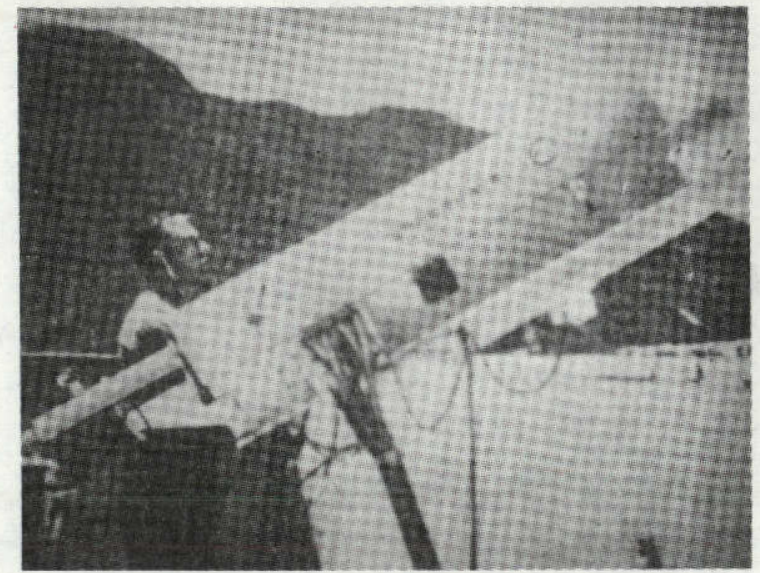

Figure 8.

Laser devices measure distances to AES and, moreover, they may be used to illuminate a satellite when it is being photographed against a star background. However, in the latter case a considerable increase of emissivity is required. Therefore, the laser devices used by the United States (Figure 8), France and Japan (a total of nine units) up to 1971 for geodesic purposes were used mainly for ranging measurements. When designing laser units, the fact is taken into account that the effective range of the system is proportional to the fourth power of emitted energy, inversely proportional to the square root of the beam width, and directly proportional to the square root of the diameter of the receiver aperture.

The divergence (width) of the laser beam of the transmitter is established as a function of the accuracy of predicting AES motion and the accuracy of laser guidance. Laser units now have a beam divergence from $0.5^{\prime}$ to $20^{\prime}$. This angle may be changed during the operation of some units, for example, on the Japanese 
device. The power of the laser varies from 10 to $50 \mathrm{~mW}$, pulse length - from 10 to 60 nanoseconds, and pulse energy - from 0.5 to $7.5 \mathrm{~J}$.

A laser unit includes a laser transmitter (ruby lasers with $\lambda=0.694$ microns are used), a recelver, a platform and a system for measuring and recording the results. The measuring process reduces to determining the time interval of passage of a light pulse from the device to the AES return. Laser guidance on the AES may be accomplished visually with the aid of a sighting device or by a program using previously established ephemerides. At the moment the laser pulse leaves the transmitter, a frequency cycle counter of $100 \mathrm{MHz}$ or $1 \mathrm{GHz}$ is triggered. The counter is closed at the moment the reflected pulse enters the receiver. The time interval $\tau$, measured in this manner, makes it possible, by knowing the speed of light $c$, to calculate the range

$$
\rho=\frac{1}{2} c \tau
$$

Range measurement accuracy is mainly determined by three factors:

- by the steepness of the front and the length of the signal returned from the AES;

- by the resolving power of the time interval counter;

- by the correct calculation of the variation of the speed of light in the atmosphere.

Because of the low power of the signal returned from the AES, only 1ts presence rather than its shape is often established. Therefore, the pulse length is of great importance to increase measurement accuracy; the less it is, the less is the error of 
recording the returned pulse. It is assumed that the transmitted and returned signal, which provides a range measurement with an accuracy of $\pm 0.6 \mathrm{~m}[59,60]$, may be made to agree by increasing the power and decreasing the pulse length.

The resolving power of the counter depends on the frequency of Its generator, by which the number of cycles from transmission to reception of the pulse is calculated. The counters in modern laser devices operate from generators of $100 \mathrm{MHz}$ or $1 \mathrm{GHz}$, and accordingly, their resolving power (scale division) comprises 10 or 1 nanoseconds ( 1.5 or $0.15 \mathrm{~m}$ ). It is assumed that the resolving power of the counters may be increased up to 0.1 nanoseconds and, consequently, the range measurement error may be reduced from $0.015 \mathrm{~m}$.

Range measurement accuracy is affected by the atmosphere, but the effect of this factor may be reduced to $0.15 \mathrm{~m}$ at the moment of observation if adequate consideration is given to temperature and pressure.

Thus, taking into account the main error sources, we may assume that an accuracy of measuring distance to AES of \pm 0.6 $0.7 \mathrm{~m}$ may be achieved. However, laser devices are fixed and, obviously, unlike photographic cameras, may be used for long periods at a few space triangulation points for scaling.

The simplest radio engineering apparatus for observation of AES are Doppler devices. They measure the Doppler frequency shift, caused by motion of the AES with respect to the observation point. The devices consist of a receiver for the frequency emitted by the sateliite, a highly stable ground-based generator and recording devices. The principle of operation of the devices is based on comparing the frequency received from the AES with 
that of the ground-based generator and in deriving the Doppler shift from their difference in the form of numerical characteristics $(n)$ at small time intervals $(\tau)$. These characteristics are proportional to the variation of distance $(\Delta \rho)$ to the AES during time $\tau$

$$
\Delta \rho=\frac{c}{f} n
$$

where $c$ is the propagation velocity of radio waves; and $f$ is the frequency with respect to which the numerical characteristic is measured.

If the measurement of the characteristic is related to the average moment, we may obtain the value of the radial component of the topocentric velocity of the AES $(\dot{\rho})$

$$
\dot{\rho}=\frac{c}{f \tau} \dot{n} \text {. }
$$

Due to the simplicity and small dimensions of the device, Doppler units of different designs are widely used in observation of AES for different purposes, including those for solution of geodesic problems.

As in laser units, measurement accuracy depends primarily on the correct allowances for radiowave propagation conditions, the stability of generators being used, and the resolving power of the counters. At present, an accuracy of receiving $\dot{\rho}$ up to $0.02 \mathrm{~m} / \mathrm{sec}$ has been reached [71]. 
CHAPTER 2

COORDINATE SYSTEMS AND THEIR TRANSFORMATION

Among the many coordinate systems used in space geodesy, we 121 shall consider only those which are required for the following discussion.

\section{Stellar Coordinate Systems}

Stellar coordinate systems are spherical. In connection with the fact that the diurnal parallax of all stars is essentially equal to zero, the origin of these systems may be placed at any point both inside (including the center of mass) and on the surface of the Earth, the spherical coordinates which characterize the bearing toward the star being varied negligibly.

The coordinate surfaces of these systems are: a sphere of unit radius $(R=1)$; conical surfaces ( $\delta=$ const) with an apex at the origin of the coordinates and with an axis parallel to some position of the Earth's rotational axis; and half-planes limited by the axis of the conical surfaces.

If the initial half-plane passes through the point of the vernal equinox, the system does not take part in the diurnal rotation of the Earth and is fixed in this sense. Such a coordinate system is usually employed in practical astronomy and is called a secondary equatorial system. The bearings to stars in this system are given by right ascension $\alpha$ and declination $\delta$ (Figure 9). 


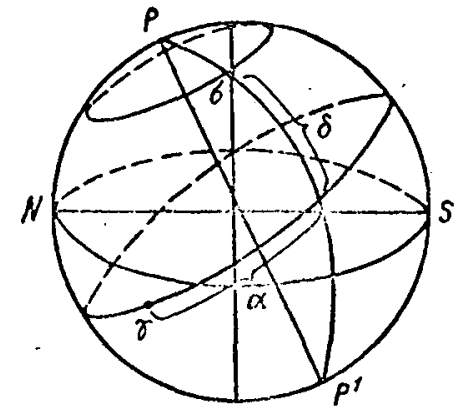

Figure 9.

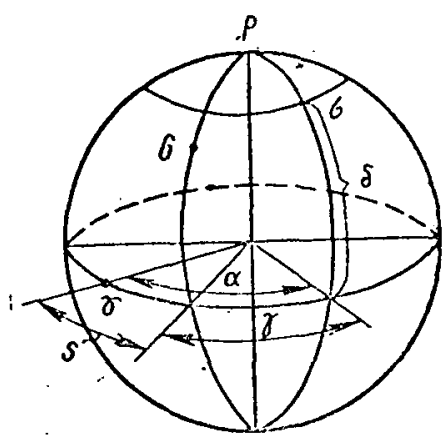

Figure 10.

Based on to which position of the equator and which equinox the coordinate system is related, the following are distinguished:

- the instantaneous system determined by the instantaneous equator and the true point of the vernal equinox;

- the average system for the epoch $T$, in which the mean equator and the point of the vernal equinox to this epoch are used.

A system for a specific epoch $T_{0}$ is fixed by the stellar coordinates in the catalog. Variation of them in time is caused only by the natural motions of the stars.

An instantaneous stellar system is not an inertial system. Because of the fact that it is fixed in space at every moment by the direction of the Earth's rotational axis, which varies under $/ 22$ the effect of precession and nutation, the stellar coordinates in this system vary continuously (the stars shift their position by 20 " per year).

When considering problems of space triangulation, a stellar coordinate system must be used which differs from an instantaneous system in the fact that its initial half-plane does not pass through the point of the vernal equinox, but is located parallel 
to the instantaneous plane of the Greenwich meridian. This system participates in the diurnal rotation of the Earth and in this sense is a rotational system. This system corresponds to the primary equatorial Greenwich coordinate system used in astronomy. The bearings toward stars in this coordinate system are given by the Greenwich horary angle $t$, or by angle $\gamma$ opposite in sign to it, and by declination $\delta$ (Figure 10).

Along with equatorial coordinate systems, a coordinate system is used in which the coordinate planes are the planes of the horizon and the meridian of the point. This coordinate system is called a horizontal system. Direction in this system is determined by the zenith angle $z$ and azimuth $A$.

\section{Transformation of Stellar Coordinate Systems}

Satellite coordinates are calculated in the stellar system which includes reference stars from the results of photographic observations. For geodesic treatment of such measurements, it is necessary that the mutual position of the coordinate axes of the stellar and ground coordinate systems be correlated.

The coordinates of reference stars are selected from star catalogs, compiled in the coordinate system given for a specific epoch $\left(1900.0+T_{c}\right)$ - the catalog epoch. Because of the fact that the Earth's rotational axis does not maintain a constant direction in space with respect to stars, but varies under the effect of precession, secular rotation, and nutation - the number of periodic oscillations - the problem arises of recalculating (reduction) the stellar coordinates from the catalog system to an instantaneous system for the observation date $\left(1900.0+\mathrm{T}_{\mathrm{H}}\right)$. 
The procedure for such reduction in a matrix notation of formulas more convenient for use by computers, is presented in the article of N. I. Idel'son [23] and in a number of more recent investigations $[11,46]$, etc.

Let us select from the coordinate catalog any star $\left(\alpha_{c}^{(i)}\right.$, $\left.\delta_{[\mathrm{c}}^{(1)}\right)$. Let us correct them for natural motions from the catalog epoch to the moment of observations, and let us write the direction cosines of the bearing toward the star

$$
\begin{aligned}
& l_{\mathrm{c}}^{(i)}=\cos \alpha_{l \mathrm{c}}^{(i)} \cos \oint_{\mathrm{c}}^{(i)} \text {; }
\end{aligned}
$$

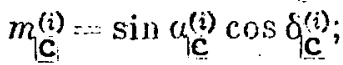

$$
\begin{aligned}
& \text { - } n_{(\mathrm{c})}^{(i)}=\sin \delta_{i c}^{(i)} \text {. }
\end{aligned}
$$

The effect of precession leads to a variation of the direction cosines, which is given by the transformation

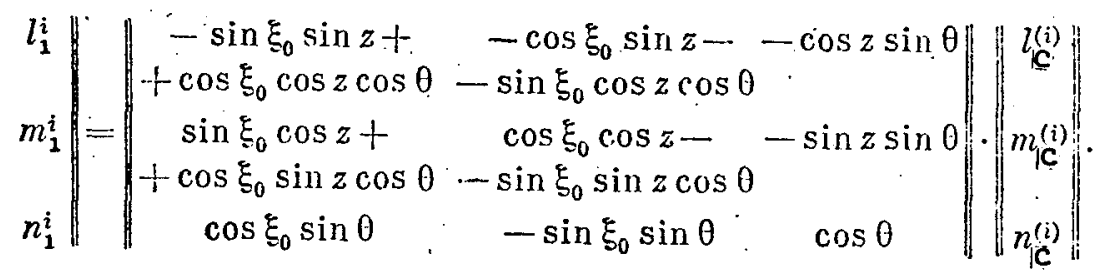

The Eulerian angles in transformation (6.2) are calculated with an accuracy to small numbers of the third order by the Newcomb-Andway expressions:*

$$
\begin{aligned}
& \xi_{0}=\left(2304^{\prime \prime}, 253+1^{\prime \prime}, 397 T_{\mid c}\right) \tau+0^{\prime \prime}, 302 \tau^{2}+0^{\prime \prime}, 018 \tau^{3} ; \\
& z=\left(2304^{\prime \prime}, 253+1^{\prime \prime}, 397 T_{\mid \vec{c}}\right) \tau+1^{\prime \prime}, 095 \tau^{2}+0^{\prime \prime}, 018 \tau^{3} ; \\
& \theta=\left(2004^{\prime \prime}, 685+0^{\prime \prime}, 853 \tilde{T}_{|c|}\right) \tau-0^{\prime \prime}, 427 \tau^{2}-0^{\prime \prime}, 042 \tau^{3},
\end{aligned}
$$

where $\tau=T_{\mathrm{H}}-T_{\mathrm{C} \mathrm{j}} T_{\mathrm{H}}, T_{\mathrm{ic}}$ and $\tau$ are given in tropic centuries, counting from the epoch of 1900.0 .

*These expressions are sometimes transformed by substitution of the epoch of a specific catalog, for example, 1950.0 . 
The effect of nutation must be taken into account to convert from the mean coordinates with consideration of precession to true coordinates. With an accuracy up to the square of small values of $\delta \psi$ and $\delta \varepsilon$, transformation of the direction cosines for nutation is calculated by the expression

$$
\left\|\begin{array}{l}
l_{l} \\
m_{l} \\
n_{l}
\end{array}\right\|=\left\|\begin{array}{ccc}
1 & -\delta \psi \cos \varepsilon & -\delta \psi \sin \varepsilon \\
\delta \psi \cos \varepsilon & 1 & -\delta \varepsilon \\
\delta \psi \sin \varepsilon & -\delta \varepsilon & 1
\end{array}\right\| \cdot\left\|\begin{array}{c}
l_{1}^{i} \\
m_{1}^{i} \\
n_{1}^{i}
\end{array}\right\|
$$

The expansions presently used for nutation in terms of longltude $\delta \psi$ and inclination $\delta \varepsilon$ are presented in Astronomical Papers, Vol. 25, p. 1, 1953, have been published since 1960 in the Astronomical Yearbook of the USSR. If we discard the terms of a series whose total effect on the accuracy of transformation $/ 24$ does not exceed $\pm 0.05^{\prime \prime}$, the formulas for calculating the nutational elements assume the form:

- the long-period portion of nutation with respect to longitude

$$
\begin{gathered}
\Delta y=-17^{\prime \prime}, 2327 \sin \Omega-1^{\prime \prime}, 2729 \sin 2(\Omega+F-D)+ \\
\div 0,20 \sin 2 \Omega+0,1261 \sin l^{\prime}-0,0497 \sin \left(2 \Omega+l^{\prime}+2 F-2 D\right)+ \\
\div 0,0214 \sin \left(2 \Omega-l^{\prime}+2 F-2 D\right)+0,0124 \sin (\Omega+2 F-2 D)+ \\
+0,0016 \sin 2 l^{\prime}-0,0015 \sin 2\left(\Omega+l^{\prime}+F-D\right)+ \\
+0,0045 \sin (\Omega-2 l+2 F)
\end{gathered}
$$

- the short-period portion of nutation with respect to longitude

$$
\begin{gathered}
d \psi=-0^{\prime \prime}, 2037 \sin 2(\Omega+F)+0,0675 \sin l-0,0342 \sin (\Omega+2 F)- \\
-0,0261 \sin (2 \Omega+l+2 F)-0,0149 \sin (l-2 D)
\end{gathered}
$$


- the long-period portion of nutation with respect to inclination

$$
\begin{gathered}
\Delta \varepsilon=\div 9^{\prime \prime}, 2100 \cos \Omega+0,5522 \cos 2(\Omega+F-D)-0,0904 \cos 2 \Omega+ \\
+0,0216 \cos \left(2 \Omega+l^{\prime}+2 F-2 D\right)-0,0093 \cos \left(2 \Omega-l^{\prime}+2 F-2 D\right)- \\
-0,0066 \cos (\Omega+2 F-2 D)+0,0007 \cos 2\left(\Omega+l^{\prime}+F-D\right)- \\
-0,0024 \cos (\Omega-2 l+2 F)
\end{gathered}
$$

- the short-period portion of nutation with respect to inclination

$$
\begin{gathered}
d \varepsilon=+0^{\prime \prime}, 0884 \cos 2(\Omega+F)+0,0183 \cos (\Omega+2 F)+ \\
+0,0113 \cos (2 \Omega+l+2 F) \\
\\
\\
\delta \psi=\Delta \psi+d \psi \\
\delta \varepsilon=\Delta \varepsilon+d \varepsilon .
\end{gathered}
$$

The arguments in the expansion of the nutation, which are fundamental Brown constants, are calculated by the formulas

$$
\begin{aligned}
& \Omega=259^{\circ} 10^{\prime} 59^{\prime \prime}, 79-1934^{\circ} 08^{\prime} 31^{\prime \prime}, 23 T+7^{\prime \prime}, 48^{T^{2}}+0^{\prime \prime}, 00807^{\prime 3} \text {, } \\
& l=296^{\circ} 06^{\prime} 16^{\prime \prime}, 59+477198^{\circ} 50^{\prime} 56^{\prime \prime}, 79 T^{\prime}+33^{\prime \prime}, 097^{\prime 2}+0^{\prime \prime}, 05187^{\prime 3} \text {, } \\
& l^{\prime}=358^{\circ} 28^{\prime} 33^{\prime \prime}, 00+35999^{\circ} 02^{\prime} 59^{\prime \prime}, 10 T^{\prime}-0^{\prime \prime}, 51^{\prime} T^{2} \ldots-0^{\prime \prime}, 01207^{\prime 3} \text {, } \\
& F=11^{\circ} 15^{\prime} 03^{\prime \prime}, 20+183202^{\circ} 01^{\prime} 30^{\prime \prime}, 51^{\prime} T^{\prime}-11^{\prime \prime}, 56 T^{\prime}-0^{\prime \prime}, 00127^{\prime}{ }^{3} \text {, } \\
& D=350^{\circ} 4^{\prime} 1^{\prime} 14^{\prime \prime}, 95+445267^{\circ} 06^{\prime} 51^{\prime \prime}, 18 T^{\prime}-5^{\prime \prime}, 17 T^{2}+0^{\prime \prime}, 0068^{\prime 3} \text {. }
\end{aligned}
$$

As a result of the transformations carried out, we calculate $/ 25$ the true stellar coordinates $\alpha^{i}$ and $\delta^{i}$ 


$$
\begin{aligned}
& a_{i}=\operatorname{arctg} \frac{m_{l}}{l_{i}}, . \\
& \delta_{l}=-\operatorname{arctg} \frac{n_{t}}{V=\left(m_{i}\right)^{2}-1 \cdot\left(l_{l}\right)^{2}},
\end{aligned}
$$

1.e., the angles characterizing the direction toward the star with respect to the Earth's instantaneous rotational axis' (angle $\delta$ ) and in the plane of the instantaneous equator with respect to the true point of the vernal equinox (angle $\alpha$ ).

Conversion from a secondary: equatorial to a horizontal coordinate system is accomplished by rotation around the Earth's rotational axis at the angle $s$, equal to the local sidereal time of the point, and angle $\varphi$, equal to the latitude of the point, by formulas

$$
\operatorname{tg} A=\frac{\cos \delta \sin s}{\sin \delta \cos \phi+\cos \delta \sin \varphi \cos s},
$$

When converting from a primary equatorial system to a horizontal system, rotation by angle $s$ is replaced by rotation by angle $\lambda$, equal to the longitude of the point.

\section{Geodetic Coordinate Systems}

The position of the point in a geodetic coordinate system is determined by the altitude $H$ above the accepted reference ellipsold, latitude $B$ and longitude $L$. Geodetic latitude is calculated as the angle formed by the normal to the ellipsoid surface with the plane of its equator, and geodetic longitude - the dihedral angle between the planes of the initial meridian and the meridian of the given point. 
Such an ellipsoidal coordinate system is used in processing ground geodetic measurements. A system of three-dimensional rectiInear coordinates $X, Y, Z$ is more convenient in space triangulation, which in its essence is three-dimensional and is not physically connected to any reference surface. Transformation of ellipsoidal geodetic coordinates to rectilinear coordinates is accomplished by the formulas

$$
\begin{aligned}
& X=(N+I I) \cos ^{r} B \cos L, \\
& Y=(N+I I) \cos B \sin L, \\
& Z=\left(\frac{b^{2}}{a^{2}} N+I I\right) \sin B,
\end{aligned}
$$

where

$$
N=\frac{a^{2}}{\sqrt{a^{2} \cos ^{2} B+b^{2} \sin ^{2} B}}
$$

$a$ and $b$ are the semi-major and semi-minor axes of the reference ellipsoid, respectively.

Conversion from $X, Y$ and $Z$ to $B, L$ and $H$ is inevitably related to iterations when c|alculating latitude $B$ and altitude H. Formulas convenient for calculations on electronic computers may be derived from (7.1) after raising the first two expressions to the second power and adding them, and after dividing the third expression by the result obtained

$$
\operatorname{tg} L==\frac{Y}{X} ; \quad \operatorname{tg} B=\frac{Z}{\left(X^{2}+Y^{2}\right)^{T_{1}}}+\frac{N e^{2}}{N+H} \operatorname{tg} B=c+d \operatorname{tg} B .
$$

Formula (7.2) permits the cycle of approximations to be organized. For the first approximation, it is assumed

$$
\tan B=c .
$$


For the second and successive approximation of the value of $B$, from the preceding approximation we calculate the values

$$
\begin{gathered}
H=\left(X^{2}+Y^{2}\right)^{1 / \bar{s}} \sec B-N, \\
N=\frac{a}{\left(1-e^{2} \sin ^{2} B\right)^{1 / 8}},
\end{gathered}
$$

after which the derivations from Formula (7.2) are repeated. The process of approximations is completed with divergence of $\Delta B$ from the next two approximations which is less than the given tolerance $\delta B_{\text {tol. }}$. Essentially three approximations are adequate in all cases to obtain tolerance $\delta B_{\text {tol }}=0.03^{\prime \prime}$.

Ellipsoidal and triangular systems are different in form, but both are geodetic by definition.

Different geodetic systems are used in the treatment of geodetic nets of different continents and even countries. Each of them has its own point of origin, in which is accomplished the "external" orientation of "its own" reference ellipsold. The vertical line at the point of origin in such an orientation is correlated with the normal to the reference ellipsoid, and the plane of the meridian of the point of origin is established parallel to the Earth's rotational axis in terms of the astronomical azimuth. In this case the vertical line and azimuth at the point of origin are calculated from astronomical observations and may be corrected for deflection of the vertical. Thus, geodetic systems are oriented for a specific epoch in a stellar coordinate system. Failure to take into account deflection of the vertical line at the point of origin, as well as to distinguish the shape and dimensions of the accented reference ellipsoid from the general Earth ellipsoid, leads . . shift of the origin of the coordinate system (the center of the reference ellipsold) 
with respect to the Earth's center of mass, without violating the parallelism of the minor axis of the reference ellipsoid with the Earth's rotational axis [29].

\section{A Common Ground Coordinate System}

Until recently the problem of establishing a common ground coordinate system was considered more in theoretical than in practical formulations. This is explained by the difficulties of making the necessary sufficiently accurate and extensive gravimetric and geodetic measurements on the Earth's surface (especially the surface of the ocean). Geodetic use of satellites (the orbital method) in combination with gravimetric methods now permits the calculation of a ground coordinate system.

Compared to geodetic systems, a ground coordinate system includes a reference surface, which may be the surface of an ellipsoid of rotation with parameters $a=6,378,165 \mathrm{~m}$ and $\alpha=$ $1: 298.25$, recommended by the $13^{\text {th }}$ Assembly of the International Astronomical Union (1967). The origin of the coordinate system is located at the Earth's center of mass. The position of the point defined as the Earth's center of mass is sufficiently stable. As Professor I. D. Zhongolovich [19] points out, a mass with an area of $10 \times 10$ equatorial degrees and altitude of $10 \mathrm{~km}$ on the Earth's surface would have to be shifted by a distance on the order of $1 \mathrm{~km}$ in order to shift it on the Earth by only 1 centimeter.

The minor axis of the common Earth ellipsoid coincides with the mean rotational axis of the Earth. The three-dimensional direction of the mean rotational axis of the Earth is fixed with respect to the stars by the coordinates of the mean pole for the mean epoch of 1900 - 1905, and its displacement with respect to 
the instantaneous pole is calculated by the International Polar Service from observations at five points, located approximately along the parallel at $39^{\circ}$ North Latitude. The invariability of the latitude of these points is naturally assumed in this case, since in the opposite case the concept of the mean pole would be very indefinite.

The plane of the initial meridian is established by determining the beginning of the calculation in a common time system.

In shape the common ground coordinate system may be elliptical $\left(B_{*}, L_{*}, H_{*}\right)$ and spatial-rectangular $\left(X_{*}, Y_{*}, Z_{*}\right)$. Conversion between them is accomplished by Formulas (7.1). It is more convenient in space geodesy to use the spatial-rectangular system.

9. Transformation of Coordinates from One Geodetic System to Another and Conversion to a Common Ground System

Establishment of a common ground coordinate system may be accomplished by a geodetic system (or several geodetic systems) by transfer of its origin to the Earth's center of mass, 1.e., by calculating the vector $\Delta r_{1}\left(\Delta X_{1}, \Delta Y_{1}, \Delta Z_{1}\right)$ of the origin of the coordinates of a geodetic system in a common ground system. If, moreover, the geodetic system has misalignment of the axes, it is first necessary to rotate it by three Euler angles $(\varepsilon, \omega, \psi)$ to achieve parallelism of the axes of the geodetic and common ground systems. In this case the radius vector of each point $r_{i}$ of the geodetic system is transformed to the radius vector $r_{1}^{\prime}, \underline{128}$ where $r_{1}^{\prime}=M r_{1}$ 


$$
M=\left\|\begin{array}{rcr}
1 & -\omega & \psi \\
\omega & 1 & -\varepsilon \\
-\psi & \varepsilon & 1
\end{array}\right\|
$$

The general transformation of coordinates of the points from a geodetic to a common ground system may now be represented by the expression

$$
r_{i}^{*}=M r_{i}+\Delta r_{i \circ}
$$

The orbital method of space geodesy permits the calculation of all six elements of transformation of (9.2), i.e., complete calculation of the orientation of the common ground coordinate system. The space triangulation method is used only to calculate the angles which characterize the inclinations of geodetic systems with respect to a common ground system, 1.e., three elements of the transformation (9.2).

The impossibility of relating the origin of coordinates of a geodetic system to the Earth's center of mass by the space triangulation method is obvious. This is determined by the fact that the angular measurements in this triangulation are essentially insensitive to transfer of the origin of coordinates, and Inear measurements are generally invariant to coordinate transformations.

If transformation of (9.2) is established for each geodetic system, the mutual shift of their origins may still be calculated from the differences $\Delta r^{\prime}{ }_{i j}$, and at the same time the problem of the relationship of geodetic coordinate systems may be solved. 
In the future, taking into account the smaliness of the possible inclinations of geodetic systems, we shall disregard them, with the exception of individual cases which will be indicated.

\section{Time Measurement Systems}

A knowledge of the precise time of observations is required for transition from a stellar coordinate system to one, rigidly coupled to the Earth. In connection with this, we shall present the brief characteristics of time measurement systems, used both in astronomy and in space geodesy.

Periodic processes, whose period is constant with a high accuracy, are used as the standards for time measurement. The Earth's rotation was the standard for many centuries, with a corresponding unit of measurement, days, and 1/86,400 part of a day - the second. However, in recent decades a whole series of seasonal, secular and irregular nonuniformities in the motion of our planet was determined. Therefore, a unit of time measurement, based on the resonance frequency of quantum transitions of cesium atoms, now is used in the International SI System. This unit is equal to 9,192,631,770 emission periods, corresponding to the transition between two super-thin levels of the basic state of an atom - a cesium isotope with a mass number of 133 in a zero magnetic field. The second calculated in this manner is very close to $1 / 86,400$ part of a day and is called an "atomic second," and the time scale determined by this unit is called "atomic time" (AT). The clocks for AT time are atomic clocks, which consist of a continuously operating quartz generator and a cesium frequency standard, which is switched on periodically to check the nominal frequency of the quartz generator. 
The high stability of an atomic time scale led to the fact that it replaced the astronomical time scale during a study of most physical phenomena, and now astronomical time is only a characteristic of the Earth's rotation. However, when investigating the position of objects in coordinate systems, rigidly coupled to the Earth, astronomical time, which determines the Earth's rotations with respect to the inertial coordinate system, should be used.

Therefore, let us dwell on astronomical time systems. As we know, the length of days is different and depends on which point of the firmament, reflecting the Earth's rotation, describes a total revolution (for which points the interval between two successive culminations is taken): the point of the vernal equinox (stellar time) and the center of the visible Sun (true solar time) or "average" Sun - a fictitious point whose motion is assumed to be uniform during the course of a year (mean solar time).

Mean solar time, counted from midnight at the Greenwich meridian, is called Universal Time. Three systems of Universal Time are distinguished.

1. TUo - Universal Time, obtained on the basis of direct astronomical calculations. If time is considered as a phase angle of the Earth's rotation in space, $\mathrm{TU}_{0}$ is the angle between the instantaneous position of the Greenwich meridian and the declination circle of the mean Sun.

2. TU1 - this is $\mathrm{TU}_{0}$ time, into which corrections are introduced for shifting of the terrestrial pole, caused by oscillation of the Earth with respect to its rotational axis. Thus, TUl may be regarded as the angle counted from the mean Greenwich meridian. 
3. TU2 - this is TUI time, into which are introduced corrections for the seasonal irregularity of the Earth's rotation. Unlike TUo and TUl time, which are not uniform due to seasonal variations in the Earth's motion, TU2 time may be regarded as uniform for a rather large time interval (up to several years, because the secular irregularities of the Earth's rotation are essentially discernible only at large time intervals). However, to solve various types of problems of celestial mechanics over prolonged time segments, strictly uniform "ephemerides" time TE with a constant unit of measurement - the second, equal to $1 / 31,556,925.9747$ part of a tropical year, beginning on 31 December 1899, was introduced. Practical calculation of TE time is carried out from observations of the orbital motion of the Moon around the Earth.

As already indicated above, the $\mathrm{TU}_{0}$ time scale is obtained from astronomical calculations. These calculations are made at a number of time bureaus and then equalized by comparing readings of time bureau clocks. Corrections to TUo time during transition to TUl time are calculated from the coordinates of the instantaneous pole, determined by the International Pole Service. Transition to TU2 and TE time is accomplished by extrapolation of corrections, derived on the basis of investigating the irregularities of the Earth's motion during preceding years. In recent years, extrapolated TU2 and TE time systems have been replaced by highly stable atomic time, which may be measured with a high degree of accuracy ( $10^{-10}$ and even more accurately) in several minutes, whereas observations over the course of a number of years are required to obtain TU2 and TE times with such accuracy.

The precise value of the difference of $\Delta T=T E$ - TUl between ephemerides and Universal Time is calculated from an analysis of observations of the Moon, and the approximate value of $\Delta T_{\text {extrap }}$ 
Is published for the current year in the astronomical yearbooks. This value was $+38.0^{\mathrm{s}}$ for 1970.5 .

When calculating transition (TAI - TU2) from approximately uniform TU2 time to atomic time in the TAl scale of the state Standard of Time and Frequency of the USSR, it was assumed that these times coincided on 1 January 1964 at $12^{h^{\mathrm{T}}} \mathrm{TU}$. This difference was about $5^{\mathrm{S}}$ by the beginning of 1970 .

It may be discerned from the above that when solving problems of space triangulation, in which one of the coordinate planes is the plane of the mean Greenwich meridian, TUl time should be used.

For problems solved by orbital methods, a system of uniform time, atomic time, should be used for integration of equations of motion. To bring atomic time into agreement with TUl time, the origin of counting in atomic time may vary periodically so that the difference in these systems does not exceed $1^{\mathrm{S}}$. Thus, the scale of such atomic time will be stepwise uniform. 


\section{CHAPTER 3}

\section{PRELIMINARY PROCESSING OF THE RESULTS OF OBSERVATIONS \\ 11. Problems of Preliminary Processing}

The results of AES observations obtained at points usually may not be directly used to adjust space triangulation. This is explained by the fact that these results sometimes do not contain numerical characteristics, as is true, for example, in photographic observations: in this case the result of observations is photographs of AES against the background of the stellar sky, subject to further measurements. Sometimes, for example, in radiotechnical measurements, the results are obtained in the form of numerical characteristics related to the elements of space triangulation by such complex mathematical functions that direct use of these measurements in space triangulation is essentially impossible.

In connection with this, the problem arises of obtaining from observational materials those numerical characteristics which are related by simple functions to the unknowns of space triangulation, which at the same time may be obtained with a sufficient degree of accuracy from the materials of direct observations. Thus, the main problem of preliminary processing is to obtain "measured values" of space triangulation with consideration of their real dependence on the results of direct measurements. Problems of preliminary processing also include analysis of the accuracy of the measured values, * determination and checking of observations

*Usually, such an analysis may be accomplished only in terms of internal conformity of measurements. 
which contain gross errors, introduction of corrections which take into account the effect of external factors and equipment corrections, and reduction of measured values to the centers of observation points.

During preliminary processing of measurement results, an attempt is made to consider all systematic equipment corrections and all corrections for external factors. However, there are a number of reasons which limit measurement accuracy. For radiotechnical measurements, this is an inaccurate knowledge of the propagation speed of electromagnetic waves in the atmosphere and their refractive index in the ionosphere and troposphere, which means the corrections for refraction are inaccurate. There are several causes which limit accuracy for photographic observations. Let us dwell briefly on some of them.

The accuracy of calculating the direction toward an object from a photograph of the stellar sky depends on the accuracy of relating the stars on the photograph to an inertial coordinate system. In order to do this, it is necessary to have a sufficient number of stars on the photograph, the positions and natural motions of which are known with a high degree of accuracy.

Fundamental star catalogs, which may provide the necessary accuracy, contain only an insignificant number of stars. Thus, for example, catalog FK-4 (Fourth Fundamental Catalog, 1963), which is the most accurate at present, contains only 1,535 stars. But, as has been determined recently, this catalog contains a systematic error of about $0.01^{\mathrm{S}}$ for right ascensions between $\alpha=$ $15^{\mathrm{h}}$ and $\alpha=18^{\mathrm{h}}$. The most complete of the fundamental catalogs, the General Catalog Boss (published in 1936) contains about 30,000 stars. It is insufficient to process the photographs of this catalog, because no more than $3-5$ stars, available in this 
catalog, impinge on the photograph of a satellite camera, usually having dimensions of $18 \times 18 \mathrm{~cm}$. It should be noted that systematic errors in the differences of coordinates of the Boss and FK-4 catalogs in right ascension ( $\alpha \cos \delta$ ) reach $0.038^{\mathrm{S}}$ and declination $(\delta) 0.37^{\prime \prime}$, and in natural motions (for 100 years) for right ascension, $0.067^{\mathrm{S}}$ and for declination, $0.48 "$ [55].

Along with fundamental catalogs, different photographic catalogs are used whose accuracy is considerably lower; this is especially true of the natural motions of stars. Moreover, there are significant systematic inconsistencies between different photographic catalogs (and even their individual parts).

In 1966 the Smithsonian Astrophysical Observatory published a star catalog which included the coordinates and natural motions of 258,997 stars from different catalogs. The positions of these stars are presented for epoch 1950.0 in the FK-4 system. All errors of the primary source catalogs entered the newly compiled catalog. Therefore, the errors in star positions for epoch 1963.0 comprise an average of $\pm 0.4 "$. However, if approximately 100,000 stars have a mean square error up to $0.3^{\prime \prime}$, more than 20,000 stars have a mean square error exceeding 0.8". And since the greater part of the error occurs due to the imprecise natural motions of stars, by 1970 the errors of star positions had increased.

Random errors of star positions may be partially eliminated by increasing the number of stars used in each photograph, but the problem of increasing accuracy may be solved only after the compilation of star catalog AGK-3. This is an important work which is being carried out by astronomers of many countries and will be completed in the future. 
Along with errors in the coordinates of reference stars, taken as.fixed reference points, the accuracy of determining the bearing toward AES is affected by a number of other factors, which may only be partially eliminated by processing. For example, errors due to the non-rigorous perpendicularity of the photographic plane to the optical axis of the camera, inaccurate knowledge of the center of projection, and radial distortion are eliminated in calculations. The effect of random errors, caused by irregular deformations of the photoemulsion layer, the negligible portion of differential refraction, etc., may be appreciably attenuated by selection of a large number of reference stars, located as closely and as symmetrically as possible to the AES image. The effect of random guidance errors on star and AES images during measurements on coordinate-measuring machines is attenuated by repeated guidance with rotation by $180^{\circ}$ of the object being measured.

However, guidance errors in cases of very blurred and extended images usually systematically distort the calculated equatorial coordinates of the satellite. Such images are obtained if the camera objective has different discernible aberrations (for example, a large coma), with coarse graininess of the photoemulsion layer, due to different photoeffects. One of the main reasons for the fact that AES images are not received as points during observations in the tracking mode, is the poor compensation for the satellite's motion. There is only one way to reduce the effect of these errors - perfection of the observation equipment and improvement of the quality of photographic materials.

The error most difficult to consider and correct is caused by the scintillation effect, which occurs due to the various turbulent motions of the atmosphere. This effect is reflected in different ways on star images, photographed under long exposures, and AES images, photographed with very short exposures. 


\section{Preliminary Processing of the Materials}

of Photographic Observations

As a result of photographic observations, AES motions with respect to reference stars at specific moments of time are recorded on the photograph. In order to obtain angles which characterize the topocentric direction toward the AES and which are "measured values" of space triangulation, the coordinates of the star and AES images on the photograph must be measured, the coordinates of reference stars must be selected from star catalogs, the time recording data must be processed and the measured plane coordinates of AES must be recalculated to equatorial coordinates for the epoch of observation.*

For this purpose, stars which will be reference points on the photograph are first selected and their coordinates are determined from the star catalog, i.e., the one-two-one congruence of the stars represented on the photograph to stars having coordinates in the catalog is established. This process is called "star identification."

Star identification is usually carried out visually with the aid of different star atlases by comparison of the star configuration on the photograph with that on the pages of the atlas. However, identification may also be accomplished with the aid of electronic computers, by comparing the difference of star coordinates in the catalog to the corresponding image of scaled differences of the coordinates measured on the photograph. In this case it is first necessary, naturally, to have the star catalog recorded in the computer memory and secondly, to know rather precisely (no more roughly than $0.5^{\circ}$ ) the equatorial coordinates

* By analogy with calculatio: of star coordinates in photographic astrometry, the complex of these operations is sometimes called satellite astrometry. 

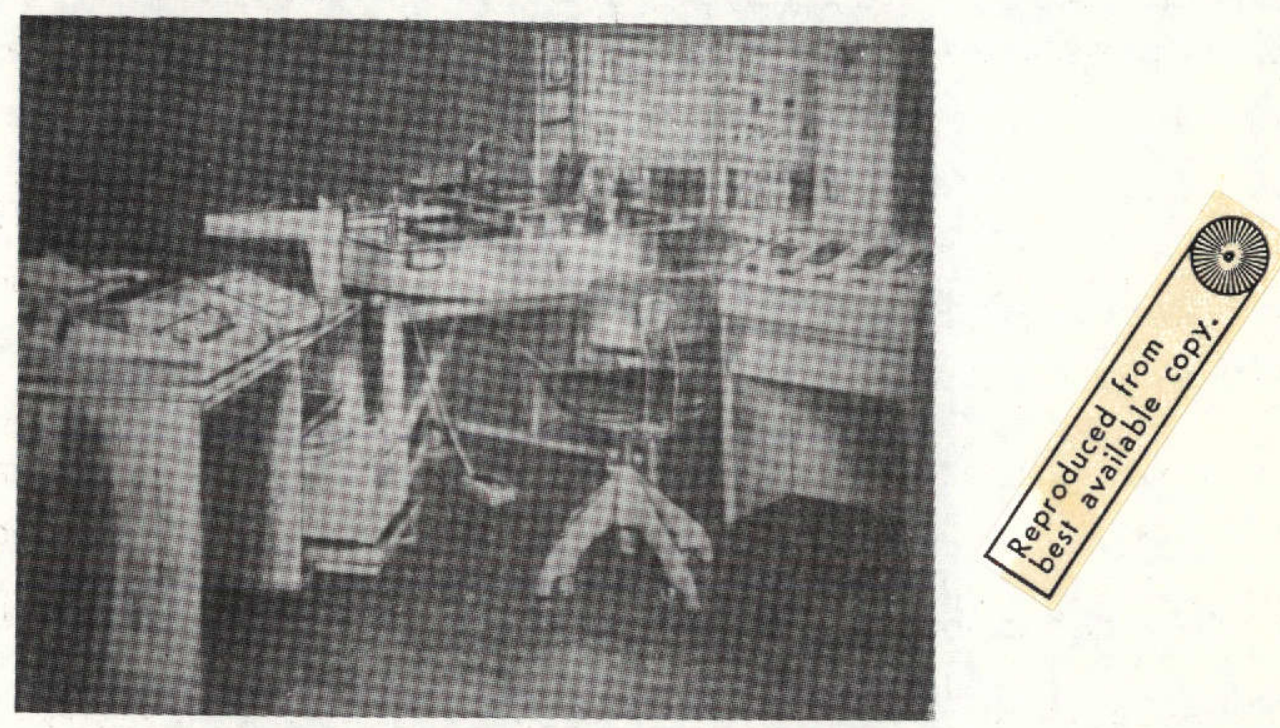

Figure 11.

of the center of the photograph. Star identification with the aid of electronic computers is accomplished by the method of sequential sorting of star coordinates and does not differ essentially from visual identification.

After the reference stars have been selected, the mutual dispositions of AES images and reference stars must be measured. The measurements are carried out on highly accurate coordinatemeasuring machines, which provide a measurement accuracy of $1-3$ microns. The most widely used machine for this purpose is the coordinate-measuring machine of the Karl Zeiss Company (GDR, Jena) the "Askorekord" (Figure 11), equipped with electronic apparatus for automatic recording of the readings and delivery of them for 135 printout and perforation.

The congruence between the equatorial coordinates of stars and the measured plane coordinates of their images on the photograph must now be established. For this purpose, errors for refraction and annual aberration should first be introduced into 
the equatorial coordinates of stars, at the moment of observations (Article 9) in order to obtain the visible coordinates of stars corresponding to their mutual disposition on the celestial sphere at the moment of observation.

Correction for refraction at the zenith distance is calculated by the formula [21]

$$
\Delta z=-58^{\prime \prime}, 20 \operatorname{tg} z+0^{\prime \prime}, 07 \operatorname{tg}^{3} z
$$

By decomposing this correction into components of right ascension and declination, we have

$$
\begin{aligned}
& \Delta \alpha=\Delta z \sec \delta \sin q, \\
& \Delta \delta=\Delta z \cos q,
\end{aligned}
$$

where $\mathrm{q}$ is the parallactic angle.

Correction for annual aberration is calculated by formulas [21]

$$
\begin{gathered}
\Delta a=C c+D d, \\
\Delta \delta=C c^{\prime}+D d^{\prime}, \\
c=\frac{1}{15} \cos \alpha \sec \delta ; \quad c^{\prime}=\operatorname{tg} \varepsilon \cos \delta--\sin \alpha \sin \delta ; \\
d=\frac{1}{15} \sin \alpha \sec \delta ; \quad d^{\prime}=\cos \alpha \sin \delta ; \\
C=-k \cos L_{\supset} \cos \varepsilon ; \quad D=-k \sin L_{\odot},
\end{gathered}
$$

where $k$ is the constant of annual aberration and $L_{\odot}$ is the longitude of the Sun at the moment of observation.

Correction of the star coordinates for diurnal aberration may be ignored due to its smallness. 
Let us now turn to consideration of the relationship between the equatorial coordinates of stars $(\alpha, \delta)$ and their plane rectangular coordinates on the plate. The image on the plate is the central projection from the second center point of the objective. Let us introduce on the plate a coordinate system with origin $\overline{0}$ at the opt1cal center of the photograph.*

Let us take the projection of the hour circle of the point on the celestial sphere with coordinates $\alpha=A$ and $\delta=D$, which is projected into the optical center, as the $\eta$ axis, and the perpendicular to the $n$ axis at the origin of the coordinates as the $\xi$ axis. Let us determine the positive direction of the axes by the increment of declination and right ascension, respectively. The coordinates on a photograph with such a direction of the axes are called ideal or standard in astrometry. Let us consider the projection $\bar{s}$ of any star $S$ with equatorial coordinates $\alpha, \delta$. The distance $\overline{\mathrm{S}} \overline{0}$ on the photograph will be

$$
\bar{S} \bar{O}=F \operatorname{tg} \omega,
$$

where $\omega$ is the angle at the center point of the objective, equal to the arc on the celestial sphere between the star and the point corresponding to the optical center.

Coordinates $\xi$ and $n$ of the star image will be

$$
\begin{aligned}
& \xi=F \operatorname{tg} \omega \sin p \\
& \eta=F \operatorname{tg} \omega \cos p
\end{aligned}
$$

where $p$ is the position angle (Figure 12 ) and $F$ is the focal distance of the objective.

*The base of a perpendicular, drawn from the second point of the objective to the plane of the photograph, is called the optlcal center of the photograph. 


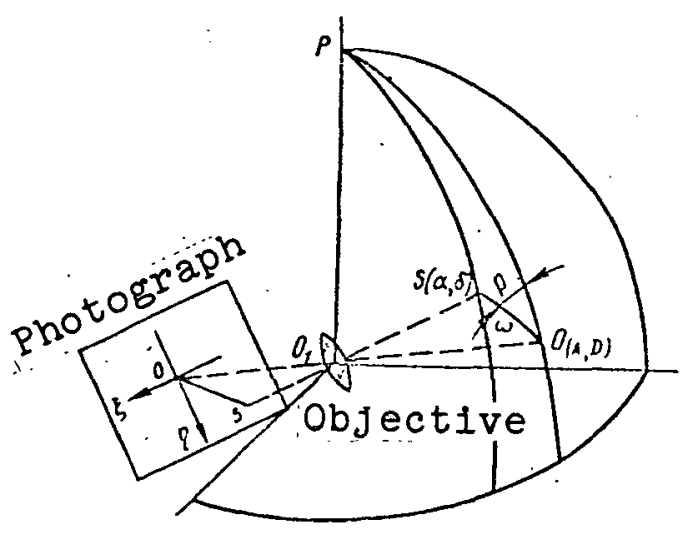

Figure 12.

Let us consider the images of the hour circles of points $S$ and $O$ on the celestial sphere. From spherical triangle sPo, taking the fact into account that $\widetilde{S O}=\omega$, $\widetilde{S P}=90^{\circ}-\delta$, and $\widetilde{O P}=90^{\circ}-\mathrm{D}$, we have

$$
\sin p \sin \omega=\sin (\alpha-A) \cos D
$$
$\cos p \cos \omega=\sin \delta \cos D-\cos \delta \sin D \cos (\alpha-A)$.

Angle $\omega$, in terms of the property of central projection, is equal to the angle between the direction toward the star and that toward point 0 .

The cosine of angle $\omega$ is found as the sum of the products of the direction cosines for the directions $O_{1} S$ and $O_{2} O_{\text {. }}$

$$
\begin{gathered}
\cos \omega=\cos \delta \cos D \sin a \sin A+\cos \delta \cos D \cos \alpha \cos A+ \\
\quad+\sin \delta \sin D=\cos (\alpha-A) \cos \delta \cos D+\sin \alpha \sin D
\end{gathered}
$$

Dividing the right and left sides of Expressions (12.6) by (12.7), after simple transofrmations, we obtain

$$
\begin{aligned}
& \xi=F \frac{\operatorname{ctg} \delta \sin (\alpha-A)}{\operatorname{ctg} \delta \cos (\alpha-A) \cos D+\sin D} \\
& \eta=F \frac{\cos D-\operatorname{ctg} \delta \cos (\alpha-A) \sin D}{\operatorname{ctg} \delta \cos (\alpha-A) \cos D+\sin D}
\end{aligned}
$$


The inverse relation is defined by expressions

$$
\begin{gathered}
a=\operatorname{arctg} \frac{\xi}{r^{\prime} \cos D-\eta \sin D}+A, \\
\delta=\operatorname{arctg} \frac{\eta \cos D+F \sin D}{F \cos D-\eta \sin D} \cos (\alpha-A) .
\end{gathered}
$$

However, the coordinates of stars and AES, measured on the photograph, will not be ideal coordinates - the origin in the coordinate-measuring machine does not coincide with the optical center of the photograph, and the coordinate axes do not colncide with the directions of the axes of idea coordinates. Therefore, in order to convert from measured $(x, y)$ to idea $(\xi, \eta)$ coordinates, it is necessary to rotate and shift the system

$$
\begin{aligned}
& \xi=a_{0}+x \cos \theta-y \sin \theta, \\
& \eta=b_{0}+x \sin \theta+y \cos \theta .
\end{aligned}
$$

Essentially, the relationship between ideal and measured coordinate systems is more complex, because central projection is Idstorted considerably due to the different aberrations of the objective, inadequate clamping and non-equalizing of the film during photographing, irregular deformation of the photoemulsion layer, etc. This requires consideration of higher-order terms in the relationship between ideal and measured coordinates. Therefore, we usually use polynomials of the type

$$
\begin{aligned}
& \xi=a_{0}+a_{1} x+a_{2} x y+a_{3} y+a_{4} x^{2}+a_{5} y^{2}+a_{6}\left(x^{2}+y^{2}\right) x, \\
& \eta=b_{0}+b_{1} x+b_{2} x y+b_{3} y+b_{1} x^{2}+b_{5} y^{2}+b_{6}\left(x^{2}+y^{2}\right) y .
\end{aligned}
$$

Each reference star, for which coordinates $x, y$ are measured and ideal coordinates $\xi$ and $n$ are calculated, yields a pair of equations (12.11), which may be considered as correcting equations for calculation of coefficients $a_{1}$ and $b_{1}$. 
It is natural that no less than $10-12$ reference stars are required for a reliable calculation of the coefficients of polynomial (12.11) by the least squares method. Having calculated coefficients $a_{i}$ and $b_{i}$, the ideal coordinates of the measured positions of AES may be calculated by Formulas (12.11) and then their equatorial coordinates - by Formulas (12.9). To analyze the accuracy, $\mu$ - the error of unit weight in terms of residual deviations $v_{\xi}$ and $v_{\eta}$, the weight of each unknown, obtained simultaneously with solution of normal equations for coefficient $a_{i}$ and $b_{i}$, and the mean square errors of AES coordinates are calculated.

When the dependence of ideal and measured coordinates is linear, the described method is called Turner's method in astrometry, and when higher-order terms are used, it is called Turner's generalized method, or Turner's higher-order method.

The formulas are valid in those cases when the random errors of the positions of individual images on the photographs are greater than those of the coordinates of reference stars in the catalogs.

We may assume at present that the accuracy of star coordinates in catalogs is $11 / 2-2$ orders higher than that of the measured coordinates on the photographs. In the future, as equipment and photographic materials improve, the errors of star images on the photographs may be commensurable with the errors of star catalogs, and it will then be feasible to take into account the errors of initial reference stars. 
Along with Turner's relations between ldeal and measured coordinates, described above, other methods of calculating the ideal coordinates of AES are also used. For example, three reference stars, located at the apexes of a triangle, in approximately whose center of gravity the object is to be identified, are used in Schlesinger's method [68]. The coefficients $D_{1}$ are calculated from the measured coordinates of the images of three reference stars $\left(x_{1}, y_{2} ; x_{2}, y_{2} ; x_{3}, y_{3}\right)$ and the object to be identified $\left(x_{0}, y_{0}\right)$. Then, by using these coefficients and the ideal coordinates of reference stars $\xi_{1}, n_{1}(1=1,2,3)$, we calculate the ideal coordinates of the position of the AES to be determined from the formulas

$$
\begin{gathered}
D_{1}=\left(x_{2}-x_{0}\right)\left(y_{3}-y_{0}\right)-\left(y_{2}-y_{0}\right)\left(x_{3}-x_{0}\right), \\
D_{2}=\left(x_{3}-x_{0}\right)\left(y_{1}-y_{0}\right)-\left(y_{3}-y_{0}\right)\left(x_{1}-x_{0}\right), \\
D_{3}=\left(x_{1}-x_{0}\right)\left(y_{2}-y_{0}\right)-\left(y_{1}-y_{0}\right)\left(x_{2}-x_{0}\right), \\
D=\left(x_{2}-x_{1}\right)\left(y_{3}-y_{1}\right)-\left(x_{3}-x_{1}\right)\left(\dot{y}_{2}-y_{1}\right), \\
\xi_{0}=\frac{D_{1} \xi_{1}+D_{2} \xi_{2}+D_{3} \xi_{3}}{D}, \\
\eta_{0}=\frac{D_{1} \eta_{1}+D_{2} \eta_{2}+D_{3} \eta_{3}}{D} .
\end{gathered}
$$

Deych's method [13] is based on the linear-fractional relation between ideal and measured coordinates

$$
\begin{aligned}
& \xi=\frac{a_{11} x+a_{12} y+a_{13}}{a_{31} x+a_{32} y+1} \\
& \eta=\frac{a_{21} x+a_{22} y+a_{23}}{a_{31} x+a_{32} y+1}
\end{aligned}
$$

and uses the correcting equations

$$
\begin{gathered}
v_{\xi}=a_{11} x+a_{12} y+a_{13}-a_{31} x \xi-a_{32} y \xi-\xi, \\
v_{\eta}=a_{21} x+a_{22} y+a_{23}-a_{31} x \eta-a_{32} y \eta-\eta
\end{gathered}
$$




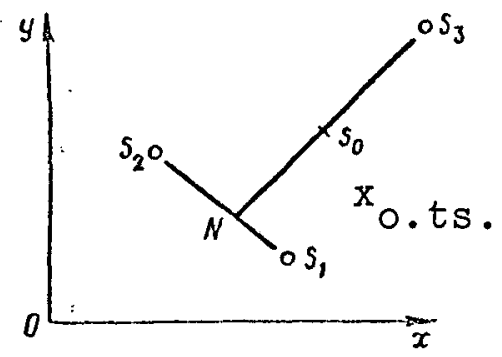

Figure 13.

There are a number of graphical and graphoanalytical methods in addition to the analytical methods of processing photographs. As an example, let us cite one method, proposed by Pulkovo astronomer A. A. Kiselev [27].

In Figure 13, $S_{1}, S_{2}$ and $S_{3}$ are the reference stars, $S_{0}$ is the position of the AES and $N$ is the auxiliary point located at the intersection of the straight line connecting both reference stars and the straight line connected the third star and the position of the AES. We initially calculate the equatorial coor- 139 dinates $\alpha_{N}$ and $\delta_{N}$ of auxiliary point $N$

$$
\begin{gathered}
\operatorname{tg}\left(\alpha_{N}-\alpha\right)=p \operatorname{tg} \frac{\Delta \alpha}{2}, \\
\operatorname{tg} \delta_{N}=\left[\operatorname{tg} \delta_{1} \sin \left(\alpha_{2}-\alpha_{N}\right)+\operatorname{tg} \delta_{2} \sin \left(\alpha_{N}-\alpha_{1}\right)\right] \operatorname{cosec} \Delta \alpha
\end{gathered}
$$

where $\alpha_{1}, \delta_{1}, \alpha_{2}, \delta_{2}$ are the equatorial coordinates of stars $S_{1}$ and $S_{2} ; \alpha_{N}, \delta_{N}$ are the equatorial coordinates of auxiliary point $\mathrm{N}$;

$$
\begin{gathered}
\Delta \alpha=\alpha_{2}-\alpha_{1} ; \\
\alpha=\frac{1}{2}\left(\alpha_{1}+\alpha_{2}\right) ; \\
p=\frac{1-\frac{m}{n}\left(1+\frac{\Delta r \bar{r}}{F^{2}}\right) \frac{\cos \delta_{2}}{\cos \delta_{1}}}{1+\frac{m}{n}\left(1+\frac{\Delta r \bar{r}}{F^{2}}\right) \frac{\cos \delta_{2}}{\cos \delta_{1}}}
\end{gathered}
$$

$F$ is the focal distance of the camera;

$$
\begin{gathered}
\bar{r}=\frac{1}{2}\left(r_{1}+r_{2}\right) ; \\
\Delta r=r_{2}-r_{1} ; \\
r_{1}=\sqrt{\left(x_{1}-x_{0} \cdot t s\right)^{2}+\left(y_{1}-y_{0} \cdot t s\right)^{2}} ; \\
r_{2}=\sqrt{\left(x_{2}-x_{0} \cdot t s_{1}\right)^{2}+\left(y_{2}-y_{0} \cdot t s\right)^{2}} ; \\
p=\frac{y_{1}\left(x_{0}-x_{3}\right)+y_{0}\left(x_{3}-x_{1}\right)+y_{3}\left(x_{1}-x_{0}\right)}{y_{3}\left(x_{0}-x_{2}\right)+y_{0}\left(x_{2}-x_{3}\right)+y_{2}\left(x_{3}-x_{0}\right)} .
\end{gathered}
$$


After the coordinates of point $N$ have been found, the coordinates of the AES position $\left(S_{0}\right)$ are found from points $S_{3}$ and $N$ in a like manner.

Having obtained the ideal coordinates of the AES by one of the above-described methods, its equatorial coordinates may be calculated from Formulas (12.9). The equatorial coordinates of the AES w1ll be in the same system as those of the reference stars. Because of the mutual motion of the AES and the Earth, the bearings toward the AES do not undergo annual aberrational displacement; therefore, the coordinates of the AES, obtained from the visible coordinates of stars, will be true, distorted only by the effect of refraction. The aberration caused by motion of the AES with respect to the observer is usually calculated by introduction of correction at the moment of observation, similarly to planetary aberration (Section 14). In order to eliminate the effect of refraction, we should introduce correction for refraction with a sign opposite to that of correction, introduced into the star coordinates. Moreover, we should take the fact into account that refraction has a different effect on stars, which we may assume to be infinitely distant, and for the satellite, which is located at a finite distance. The correction of the zentth angle of the satelilte $\Delta_{z}$ will be

$$
\Delta z=58^{\prime \prime}, 20 \operatorname{tg} z-0^{\prime \prime}, 07 \operatorname{tg}^{3} z-\frac{481^{\prime \prime}, 6}{d} \operatorname{tg} z \sec z
$$

where $d$ is the distance to the AES in kilometers. The last term of this formula is called the "refraction parallax."*

*In Weiss's investigation of 1960 [11], the coefficient is assumed equal to $435.0^{\prime \prime}$ rather than $481.6^{\prime \prime}$, and the last term of formula has the form $\left(435^{\prime \prime} / d\right)$ tg $z \sec z\left(i-e^{0.1385 d} \cos z\right)$. This divergence is negligible within the accuracies of the AES direction obtained at present. 
In some cases [46], visible rather than true coordinates of reference stars, and their average values for the catalog epoch are used. Only the natural motions of stars are taken into account and only equatorial coordinates of the AES for the same epoch are obtained. In these cases, the coordinates of the AES are reduced to their true position. Computational operations are reduced somewhat with this method, but residual errors may occur due to the differential effect of refraction and aberration.

Besides the motion of the Earth as a solid, which is taken into account by reduction to true positions, the effect of motions of the poles in the Earth's mass should be taken into account, and corrections are introduced into the measured equatorial coordinates of the AES due to variation of the pole by formulas similar to the formulas for correcting latitude and longltude [21].

$$
\begin{aligned}
& \Delta a=\Delta \gamma=-[y \cos \gamma-x \sin \gamma] \operatorname{tg} \delta, \\
& \Delta \delta=\quad-x \cos \gamma-y \sin \gamma,
\end{aligned}
$$

where $x, y$ are the coordinates of the instantaneous pole.

At observation points, cases may be encountered when they use different equipment for observations of AES, spaced at some distance from each other. In this case, the problem arises of reducing the measurements to a single point, used as the center of the observation point. For such reduction, the elements of reduction, similar to the centerings in ordinary geodetic or astronomical measurements, must be known with only the difference that, besides measurements of horizontal distances, excesses should also be measured without fall. We shall assume that all the required measurements have been completed and the differences 
of the rectangular geocentric coordinates ( $u, v, w$ ) of equipment for observation of AES and the center of the observation point have been calculated.*

Let us find the extent to which the "measured values" of space triangulation - the equatorial coordinates of AES change upon conversion from an observation point with coordinates $X, Y, Z$ to a point with coordinates $X+U, Y+v, Z+W$. After differentiating (15.2), we obtain, after elementary transformations of correction for centering,

$$
\begin{gathered}
\dot{c_{\gamma}}=\frac{u \sin \gamma}{\rho \cos \delta}-\frac{v \cos \gamma}{\rho \cos \delta}, \\
c_{0}=\frac{u \cos \gamma \sin \delta}{\rho}+\frac{v \sin \gamma \sin \delta}{\rho}-\frac{w \cos \delta}{\rho} .
\end{gathered}
$$

As can be seen from Formulas (12.18), distance $\rho$ must be known to calculate corrections to equivalent coordinates $\alpha$ and $\delta$. Since the distance's between different installations are usual|y small, it is sufficient to know the value of $p$ approximately.**

\section{Preliminary Processing of the Results of} Doppler and Laser Measurements

As in photographic observations, those numerical character1stics which are further called the "measured values" of space triangulation, may not be immediately obtained from direct measurements in radio-technical observations. Only certain parameters of the propagating radio signal (a light signal in the

*When calculating $u, v$, w, it is unnecessary to know the coordinates of the center or of the equipment, but it is important only to obtain the correct increments of the coordinates.

** In some cases, measurements may be included in equalization which are not reduced to the centers of observation points, but the coordinates obtained are corrected for values of $u, v$, w after completion of equalization. 
case of laser observations) are recorded during observation. Such parameters may be: variation of signal frequency received from the AES, compared to some reference frequency of the ground observation point; phase delay of the signal relayed by the AES; and phase difference of the signal recelved by two spaced antennas. The following may be obtained from these measured parameters: in the first case - the topocentric radial velocity of the AES and the difference of distances to two positions of the AES; secondly - the range to the AES; and third - the angle between the bearings from the AES to the antennas.

In the process of measurements, the signal coming from the AES, after a number of transformations, is usually fed into the recording device, which glves the corresponding numerical characteristic. Further processing of observations consists in decoding the readings of the recording device, calculation of the preliminary values of the measured parameter from the numerical characteristics, and their elimination by equipment corrections and corrections for refraction in the atmosphere, Ionosphere and troposphere. The nature of numerical characteristics and the method of preliminary processing depend on the method of signal transmission and the recelving apparatus used.

The distance $\rho$ to the AES is obtained directly as a result of laser observations. However, this distance is related to specific values of atmospheric temperature $T_{0}$ and pressure $p_{0}$. Therefore, during preliminary processing, it is corrected by corrections for $\Delta \mathrm{T}=\mathrm{T}-\mathrm{T}_{0}$ and $\Delta \mathrm{p}=\mathrm{p}-\mathrm{p}_{0}$

$$
\Delta \rho=-\frac{a+b \Delta p \Delta T^{-1}+c H}{\sin h+10^{-3} \operatorname{ctg} h}
$$

where $a, b, c$ are constant coefficients; $T$ and $p$ are the temperature and pressure measured at an observation point at the moment 
of observation with an accuracy up to $0.1^{\circ}$ and $0.2 \mathrm{~mm} \mathrm{Hg}$, respectively, and $T_{0}$ and $p_{0}$ are the initial values of the same parameters; $h$ is the angular elevation of the AES above the level of the observation point; and $\mathrm{H}$ is the helght of the observation point above sea level.

Moreover, correction for equipment delays is introduced into the measured range. If necessary, the measured range is reduced to the center of the obervation point by formula

$$
c_{p}=u \cos \gamma+v \sin \gamma+w \sin \delta
$$

The recording devices of Doppler equipment usually yield the numerical characteristics, equal to the Doppler frequency shift within a specific calculated time interval.* As already Indicated above, this shift is proportional to the difference of the distances between the positions of the AES at the beginning and end of the computational interval. Therefore, preliminary processing is reduced.to calculating the difference of distances $\Delta \rho$ within the calculated time interval from the numerical characteristic $N$, or to obtaining $\dot{p}$ - radial velocity, which may be accomplished by dividing the difference of the distances by the calculated time interval. Moreover, in view of the non-linearity of variation of radial velocity, the value of $\dot{p}$ obtained will not be related to the average of the calculated interval. The values of $\Delta \rho$ and $\dot{\rho}$ obtained should be corrected for refraction of the radio beam in the ionosphere and troposphere and for the relativistic effect [25]. The latter two corrections are sometimes disregarded [47], and two coherent frequencies are used to

*For example, this interval is equal to 10 seconds for the French Diadem satelilte. 
eliminate corrections for the lonospheric refraction from the satellite. Thus, for example, the Diadem satellite emits two frequencies: $F_{150}=150 \mathrm{MHz}$ and $\mathrm{F}_{400}=400 \mathrm{MHz}$. The frequencies recelved at the observation points are equal to

$$
\begin{aligned}
& f_{400}=\dot{F}_{400}+\Delta F_{400}+\frac{k}{F_{400}}+\varepsilon_{1}, \\
& f_{150}=F_{150}+\Delta F_{150}+\frac{k}{F_{150}}=\varepsilon_{2},
\end{aligned}
$$

where $\mathrm{k} / \mathrm{F}$ is correction for ionosopheric refraction of first order; $F_{400}$ and $F_{150}$ are the emitted frequencies; $f_{400}$ and $f_{150}$ are the frequencies used; $\Delta F_{400}$ and $\Delta F_{12.0}$ is the Doppler frequency shift; and $\varepsilon_{1}$ and $\varepsilon_{2}$ are corrections in frequency for different equipment and external factors.

The frequencies in the receiver are equalized and a mixed frequency is fed to the recording device, equal to $f_{400}-$ $(3 / 8) f_{150}$, free of the effects of lonosopheric refraction

$$
\begin{gathered}
f_{400}-\frac{3}{8} F_{150}=F_{400}+\Delta F_{400}-\frac{9}{64}\left(F_{400}+\Delta F_{400}\right)+\frac{k}{F_{400}}- \\
-\frac{\frac{3}{8} k}{\frac{3}{8} F_{400}}+\varepsilon_{1}+\varepsilon_{2}=\frac{55}{6}\left(F_{400}+\Delta F_{403}\right)+\varepsilon_{1}+\varepsilon_{2} .
\end{gathered}
$$

One of the important stages of preliminary processing of Doppler measurements is calculation of the so-called frequency substitution error. The fact is that numerical characteristics $\mathrm{N}$ are recorded at ground observation points, which are equal to

$$
N=C\left(f \Delta t-f^{\prime} \Delta t^{\prime}\right) \text {, }
$$

where $C$ is a coefficient dependent upon frequent multiplication in the ground equipment; $f$ is the frequency emltted by the satellites; $f$ is the frequency of the standard generator at the 
observation point; $\Delta t^{\prime}=10^{\mathrm{S}}$. Is the measured interval used to obtain a single numerical characteristic; $t^{\prime}{ }_{f}$ and $t^{\prime}{ }_{D}$ is the time of the beginning and end of the measured interval at the observation point; $t_{f}$ and $t_{D}$ are the corresponding $t_{f} f_{f}$ and $t_{D} t_{i m e}$ of the beginning and end of frequency emission from the AES.

Frequencles $f$ and $f^{\prime}$ are not known precisely due to instability of the generators, but only their nominal values $f_{\mathrm{H}_{\mathrm{H}}}$ and $\mathrm{f}^{\prime} \mathrm{l}_{\mathrm{H}}$ are known. Therefore, the following corrections should be introduced into the measured value of $\mathrm{N}$

$$
\Delta f_{f}=\left(f-f^{\prime}\right) \Delta t^{\prime}-\left(f_{n}-f_{n}^{\prime}\right) \Delta t^{\prime}
$$

The difference of $\left(f_{n}-f^{\prime}\right)$ is called frequency substitution or the frequency pedestal. Because of the slight decrease in frequencies of the ground and on-board generators during small time intervals, it is assumed that correction of frequency substitution $\Delta f_{f}$ is constant during the measurement session. This value is calculated if it cannot be determined by the apparatus method, simultaneously with preliminary refinement of the orbital parameters of the AES.

If necessary, the derived values of $\Delta \rho$ should be corrected for reduction to the observation point center by the formula

$$
c_{\Delta 0_{13}}=u\left(\cos \gamma_{1}-\cos \gamma_{2}\right)+v\left(\sin \gamma_{1}-\sin \gamma_{2}\right)+w\left(\sin \delta_{1}-\sin \delta_{2}\right)
$$

14. Processing of Time Recording Data

An independent process of preliminary processing is calculation of the time of observation of the AES. 
Only simultaneous (synchronous) observations of the AES from two or more observation points are used for construction of space triangulation. Synchronization of observations is provided by transmission of a brief light flash or, in the case of observation $/ 44$ of passive AES, by reduction of the time-overlapping observations to a single moment, called the synchronous moment.

The time of photographic observation of AES at the observation point is understood as that moment to which the calculated equatorial topocentric coordinates of the AES are related. This time is:

- the average moment assumed for processing the star exposure when observing the light flash of an active AES by fixed cameras; and the selected synchronous moment when observing passive AES;

- the moment of transmission of the light flash from an active AES during observation with star-tracking cameras, and the selected synchronous moment during observation of passive AES.

The time of radiotechnical observations at a given observation point is understood as the moment to which the measured parameter is related. For example, this time for Doppler measurements is the middle of the calculated interval of frequency reception.

When processing any time recording data at the observation point, the scale of the instrument recording time must be compared to the radio signals of precise time. 
Since the precise time signals are transmitted in a unfform atomic time system, additional corrections for conversion to the TUl system must be introduced. These corrections are calculated from astronomical observations and are published in bulletins entitled "Standard Time at Mean Moments of Radio Signal Transmissions," published monthly by the All-Union Scientific Research Institute of Physicotechnical and Radiotechnical Measurements (VNIIFTRI). Corrections $t$ for reduction of the moments, of radio slgnal transmissions to the TUl system are given in these bulletins for all Soviet and a number of foreign radio stations. In order to convert from a TUI to a TU2 system and from a TAI system, if necessary, additional corrections of $\Delta t_{s}$ (for seasonal variation of the Earth's rotation) and (TAl ${ }_{c}$ - TU2) are given.

Due to the fact that radio waves propagate with a finite velocity, correction for standard time should be added to the correction of $\tau_{p}$ for the propagation velocity of radio waves

$$
\tau_{p} \approx \frac{d}{c}
$$

where $d$ is the distance from the transmitting station to the obsenvation point, and $c$ is the propagation velocity of radio waves.

Let us consider in more detail the problem of recording and calculating the time of photographic observations.

As indicated above, when observing flashes by star-tracking cameras, the time of production of the light flash on board the AES must be known. A time bureau at the observation points is optional in this case. For this, highly stable clocks, which control the operation of the on-board equipment and at the same time the program for transmission of the flashes [72], are 
installed on board geodetic satellites of the ANNA and GEOS type, in addition to the equipment to produce the light flashes. The radio signals from the on-board clocks of the AES are recelved by special ground time bureaus* and these signals are compared to the radio signals of precise time. Thus, calculation of the flash time reduces to comparison of three time scales: (1) the radio station transmitting the precise time signal ( $T)$; (2) the ground time bureau $\left(t_{H}\right)$; and $(3)$ the on-board clocks of the satellite $\left(t_{b}\right)$.

Corrections for the readings $t_{H}$ of the time bureau clocks to reduce them to the TUl system will be

$$
\Delta t_{H}=T+\tau+\tau_{p}+\tau_{d}-t_{H},
$$

where $\tau_{d}$ is the signal lag during passage through the time bureau receiver of the observation point.

Correction for the readings $t *{ }_{b}$, of the on-board clocks at the moment of the flash will be

$$
\Delta t{ }_{b}=t{ }_{H}^{*}+\Delta t{ }_{H}+\tau{ }_{p}^{*}+\tau{ }_{d}^{*}-\tau{ }_{b}^{*},
$$

where the superscript (*) denotes that the readout (correction) refers to the moment of the flash, and $\tau^{*}{ }_{p}$ and $\tau^{*}{ }_{d}$ are the signal passage time from the AES to the ground time bureau and the delay of the time signal in the transmitting apparatus of the AES, respectively. The time of the flash will be

$$
T^{*}=\tau_{b}^{*}+\Delta \tau{ }_{b}^{*}
$$

*This time bureau may be regarded as the time bureau of the observation point, if it is equipped with highly accurate quartz or atomic clocks, regulated systematically against the state time and frequency standard. 
If the flash is photographed by a fixed camera, the bearing from the observation point to the satellite will be rigidly coupled to the Earth and will not participate in rotation of the celestial sphere; therefore, angle $\gamma$ will not vary during the time of observations, whereas angle $\alpha$ varies by a value equal to the difference in the time of receipt of the star image and the flash. Consequently, recording the moment of the flash image receipt is not required in this case for space triangulation, but only the time of receipt of the working images of reference stars must be known, with an accuracy providing only calculation of the diurnal rotation of the Earth.

When observing a passive AES, the use of star-tracking cameras does not free one from the necessity of having high-speed shutters and time recording equipment. The time of each exposure of the satellite is determined in this case by the clocks at the observation point. The clocks are compared to the radio signals of precise time in order to reduce them to the TUl system.

As a result of simultaneous photography of passive AES, pictures containing 10 or more point images of the satelitte are recelved at a number of observation points within an overlapping time interval. The time $T$ recorded by the clocks corresponds to each such image.

In the case of observing passive AES, this method permits the coordinates of the AES, uncorrected for satellite aberration, to be calculated. Introduction of corrections for satellite aberration into the AES coordinates, obtained by the photograph, is equivalent to introduction of corrections at moment $T_{0}$. Therefore, it is possible to introduce its own correction for To for each observation point, equal to 


$$
\Delta T_{0}=-\frac{D}{c}
$$

where $D$ is the distance to the AES at the moment of observation; and $c$ is the average speed of light propagation along the path.

The coordinates $x_{0}, y_{0}$ of a fictitious flash are calculated for each photograph from moments $T$ and the observation point images of the AES measured on the coordinate-measuring machine. It is sufficient to use an approximatly by the least squares method with a third-power polynomial

$$
\begin{aligned}
& x=a_{0}+a_{1} T+a_{2} T^{2}+a_{3} T^{3}, \\
& y=b_{0}+b_{1} T^{\prime}+b_{2} T^{2}+b_{3} T^{3} .
\end{aligned}
$$

Finding coefficients $a_{1}$ and $i_{1}(1=0,1,2,3)$ and substituting synchronous moment $T_{0}$ for $T$, we calculate $x_{0}$, $y_{0}$ - the rectangular coordinates of the fictitious flash at the synchronous moment of time.

When processing AES photographs by this method, the exposure time of the AES must be known with a high degree of accuracy in order to take into account not only the effect of the Earth's rotation, but the natural motion of the satellite as well.

We note in conclusion that all the assumptions of this section apply to the space triangulation method. 
CHAPTER 4

SPACE TRIANGULATION PROCEDURE

15. Measured and Unknown Values of Space Triangulation

The main volume of measuring information for space triangulation are synchronous photographic observations of AES against the background of the stellar sky. Angles $\gamma$ and $\delta$, obtained from preliminary processing of these observations, are taken as measured values in equalizing space triangulation.

Moreover, the distances to the satellite $\rho$ or the difference of distances $\Delta \rho$ to two of its adjoining positions during one passage, which are also included in equalization of space triangulation, may be measured from separate points. These measurements are usually made simultaneously (synchronously) with the photographic measurements.

The unknowns of space triangulation are the coordinates of the observation points and positions of the AES. The former are required unknowns, because their derivation is the main purpose of space triangulation, and the latter are auxiliary unknowns. In individual cases, the AES coordinates may generally not be calculated.

The functional dependence of measured values and unknowns of space triangulation is established by Expression (2.1), which in coordinate form in the spatial rectangular coordinate system has the form 


$$
\begin{gathered}
x_{k}-X_{l}=\Delta x_{i k}=\rho_{i k} \cos \delta_{i k} \cos \gamma_{i k}, \\
y_{k}-Y_{l}=\Delta y_{i k}=\rho_{i k} \cos \delta_{i k} \sin \gamma_{i k}, \\
z_{k}-Z_{l}=\Delta z_{i k}=\rho_{i k} \sin \delta_{i k} .
\end{gathered}
$$

From (15.1), we obtain an expression for the measured values directly in terms of the unknowns

$$
\begin{gathered}
\gamma_{i k}=\operatorname{arctg} \frac{\Delta y_{l k}}{\Delta z_{i k}}, \\
\delta_{i k}==\operatorname{arctg} \frac{\Delta z_{i k}}{\sqrt{\Delta x_{i k}^{2}+\Delta y_{i k}^{2}}}, \\
\rho_{i k}=\sqrt{\frac{\Delta x_{i k}^{2}+\Delta y_{i k}^{\mathrm{e}}+\Delta z_{i k}^{3}}{3} .}
\end{gathered}
$$

The expression for the measured difference of distance is obtained for the unknowns from the two Equations of (15.3), compiled for a single observation point 1 and two AES positions $(k=1.2)$,

$$
\Delta p_{i k_{1},}=-=\sqrt{\Delta x_{i h_{1}}^{2}+\Delta y_{i h_{1}}^{2}+\Delta z_{i k_{1}}^{2}}-\sqrt{\Delta x_{i k_{2}}^{2}+\Delta y_{i k_{2}}^{2}+\Delta-\Delta z_{i k_{2}}^{2}} .
$$

\section{The Principal Elements of Space Triangulation}

Each space triangulation process may be regarded as a combination of a number of geometric elements, the main ones of which are: the vector connecting the observation point and the AES position, the plane of synchronization and the vector connecting the two observation points.

The orientation of each of these elements may be determined from the results of only photographic observations without introducing any other additional data. 
The bearing from the observation point 1 to the satellite position $k$ is calculated directly from the results of observations - angles $\gamma_{1 k}$ and $\delta_{i k}$. Moreover, it may be characterized by coordinates $\tau, m$ and $n$ of the unit vector of this bearing (its direction cosines)

$$
\begin{gathered}
l_{i k}=\cos \delta_{i k} \cos \gamma_{i k}, \\
m_{i k}=\cos \delta_{i k} \sin \gamma_{i k}, \\
n_{i k}=\sin \delta_{i k} .
\end{gathered}
$$

Each two bearings from observation points $1_{1}$ and $1_{2}$ toward the satelite position $k$ determine the plane whose vector equation has the form

$$
\left(\bar{R}_{i_{1}}-\bar{R}_{i_{8}}\right) \bar{d}_{i_{1} k}^{0} \bar{d}_{i_{2} k}^{0}=0
$$

where $\bar{R}_{i_{1}}$ and $\bar{R}_{i_{2}}$ are the radius vectors of observation points $i_{1}$ and $i_{2} ; \bar{d}_{i_{1} k}^{0}$ and $d_{i_{2} k}^{0}$ are the unit vectors of the bearings to the AES. Equation (16.2) is the result of the simultaneity or the synchronism of observations; therefore, the plane which it defines is called the synchronization plane.

In coordinate form, Equation (16.2) assumes the form

$$
A_{k} \Delta X_{i_{3} i_{2}}+B_{k} \Delta Y_{i_{1} i_{2}}+C_{k} \Delta Z_{i_{1} i_{2}}=0
$$

Coefficients $A_{k}, B_{k}$ and $C_{k}$ are calculated from the measured values using (16.1) by formulas

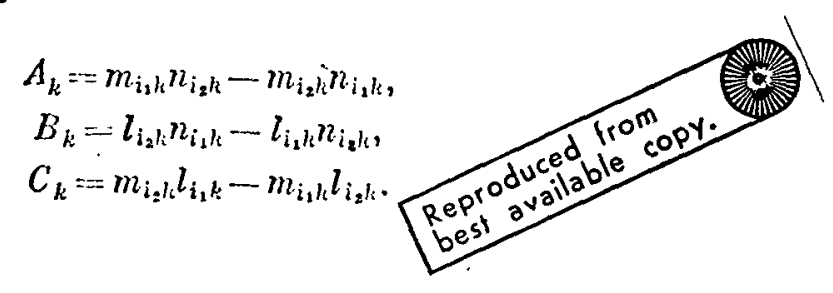


The normalizing factor of the equation of the synchronization plane is equal to the sine of the angle between unit vectors $\bar{d}_{1_{1} k}^{0}$ and $\bar{d}_{1_{2} k}^{0}$, 1.e., to the sine of the angle of intersection of bearings toward the satellite $\left(\beta_{k}\right)$. Therefore, the direction cosines of unit vector $\bar{r}_{k}^{0}$, normal to the synchronization plane, may be calculated from the formulas

$$
\begin{aligned}
& l_{k}==\frac{A_{k}}{\sin \beta_{k}}, \\
& m_{k}=\frac{B_{k}}{\sin \beta_{k}}, \\
& n_{k}=\frac{C_{k}}{\sin \overline{\beta_{i k}}}, \\
& \sin \beta_{k}=\sqrt{1-\left(l_{i_{1} l} l_{i_{2} k}+m_{i_{1} k} n_{i_{2} k} \uparrow-n_{i_{1} k} n_{i_{z} k}\right)^{2}} \text {. }
\end{aligned}
$$

If two AES positions ( $k=1,2)$ were observed from points $1_{1}$ and $1_{2}$, consequently, two synchronization planes and two unit vectors $\vec{r}_{k}^{0}$, normal to them (Figure 14 ), were obtained. The vectors $\vec{r}_{k_{1}}^{0}$ and $\vec{r}_{k_{2}}^{0}$ define the plane, the normal of which is the direction of the chord which connects the two observation points $\left(1_{1}\right.$ and $\left.1_{2}\right), 1_{1}$., the direction vector $\bar{r}_{1_{1} 1_{2}}$ of this chord is

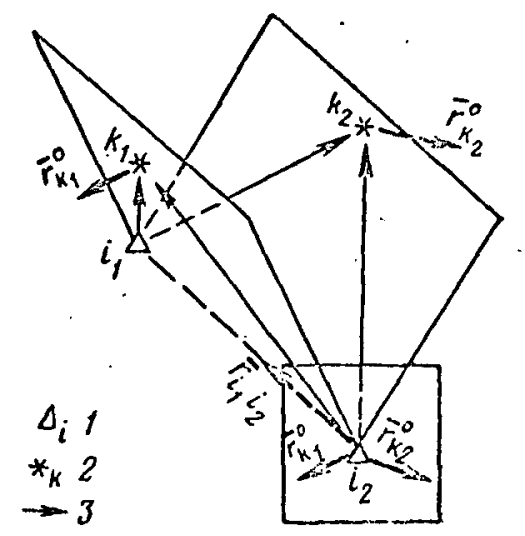

Figure 14.

1 - observation point,

2 - AES position; 3 measured direction.

$$
\bar{r}_{i_{1} i_{2}}=\bar{r}_{k_{1}}^{0} \bar{r}_{h_{2}}^{0}
$$

The direction cosines of the chord in accordance with Formula (16.5) are calculated by expressions

$$
\begin{aligned}
L_{i_{1} i_{2}} & =\frac{1}{\sin \lambda \sin \beta_{1} \sin \beta_{2}}\left(B_{1} C_{2}-C_{1} B_{2}\right), \\
M_{i_{1} i_{2}} & =\frac{1}{\sin \lambda_{1} \sin \beta_{1} \sin \beta_{2}}\left(C_{1} A_{2}-C_{2} A_{1}\right),(16.6) \\
N_{i_{1} i_{2}} & =\frac{1}{\sin \lambda \sin \beta_{1} \sin \beta_{2}}\left(A_{1} B_{2}-A_{2} B_{1}\right) .
\end{aligned}
$$


where $\lambda$ is the angle between the synchronization planes.

By analogy with $\gamma$ and $\delta$, angles $\Lambda$ and $\Phi$ are introduced which characterize the slope of the chord to the plane of the equator (angle $\Phi$ ) and in projection on the plane of the equator to the OX axis (angle $\Lambda$ ). Henceforth, we shall call them orienting angles. In accordance with Expressions (16.1), they are calculated by the formulas

$$
\begin{gathered}
\Lambda_{i_{1} i_{2}}=\operatorname{arctg}\left(\frac{M}{L}\right)_{i_{1} i_{2}}, \\
\Phi_{i_{1} i_{2}}=\ldots \operatorname{arctg}\left(\frac{N}{l^{\prime} L^{2}-1-M I^{2}}\right)_{i_{1} i_{2}} .
\end{gathered}
$$

The equation of the synchronization plane (16.2), after dividing it by $D_{i_{1} i_{2}}$ (the length of the chord), reduces to the form

$$
A_{k} L_{i_{1} i_{3}}+S_{k} M_{i_{1} i_{3}}+C_{k} N_{i_{1} i_{3}}=0
$$

Transforming (16.8) with consideration of (16.7), we obtain $/ 50$

$$
\begin{aligned}
& \operatorname{tg} \delta_{i_{1} k} \sin \left(\Lambda_{i_{1} i_{2}}-\gamma_{i_{2} k}\right)+\operatorname{tg} \delta_{i_{2} k} \sin \left(\gamma_{i_{1} k}-\Lambda_{i_{1} i_{z}}\right)+ \\
& +\operatorname{tg}\left(\Phi_{i_{1} i_{2}} \sin \left(\gamma_{i_{2}{ }^{\prime}}-\gamma_{i_{1} k}\right)=0\right. \text {. }
\end{aligned}
$$

Expression (16.9) contains the spherical coordinates of three observation points at which the bearings $1_{1} \mathrm{k}, 1_{2} \mathrm{k}$ and $1_{1} 1_{2}$ intersect the celestial sphere. It then follows from Formula (16.9) that all three points are located on a single arc of a large circle, which is the trace of intersection of the sphere with the synchronization plane. In the literature [41] this circle has been named the circule of simultaneity. A number of problems of space triangulation, for example, calculation of chord directions, may be solved both in spherical and in threedimensional rectangular coordinates. In the first case, the 
equation of the circle of simultaneity is used and in the second, the synchronization plane equation is used. Taking the fact into account that there are linear measurements in addition to angular measurements in space triangulation, as well as the simplicity of writing the formulas and calculations, a three-dimensional rectangular coordinate system is usually employed in space triangulation. Therefore, use of the circle of spontaneity will no longer be considered.

These elements, due to their orientational nature, facilitate space triangulation, if its scale is defined by even one basis - the length of one of the vectors of the observation pointsatellite or observation point-observation point.

The combination of linear measurements with synchronous photographic observations permits a direct calculation of the length of the observation point-satellite vector $\rho_{1 k}$, as well as the length of the vector connecting two observation points - the length of chord $D_{i j}$.

Thus, if the bearings toward the AES position $k$ are calculated from two observation points - the ends of chord 1 and $j-$ and, moreover, the distance from observation point $i$ to this position $\rho_{1 k}$ (Figure 15) and since

$$
\frac{D_{i j}}{\rho_{i k}}=\frac{\sin \beta_{k}}{\sin \beta_{j}},
$$

then

$$
D_{i j}=\rho_{l k} \sqrt{\frac{1-\left(l_{i k} l_{j k}+m_{i k} m_{j k}+n_{i k} n_{j k}\right)^{2}}{1-\left(l_{j k} L_{j i}+m_{j k} M_{j i}+n_{j k} N_{j i}\right)^{2}}} .
$$




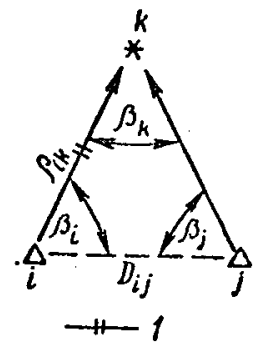

Figure 15.

1 - measured distance.

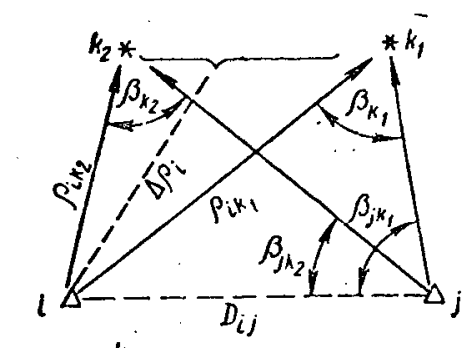

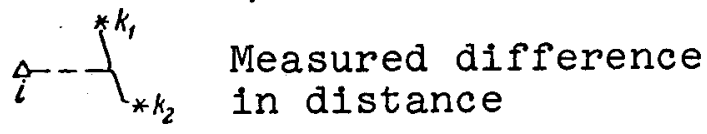

Figure 16.

If the distances to the AES position are measured from two observation points - the ends of the chord, then

$$
D_{i j}=\sqrt{\rho_{i k}^{2}+\rho_{j k}^{2}-2 \rho_{i k} \rho_{j k}\left(l_{l k} l_{j k}+m_{i k} m_{j k}+n_{i k} n_{j k}\right)} .
$$

If the bearings from the observation points - the ends of chord 1 and $j$ - toward two AES positions $k_{1}$ and $k_{2}$ and, moreover, the difference of distances to these AES positions (Figure 16) from one of the observation points (1) are calculated, then on the basis of

$$
\Delta \rho=\rho_{i k_{1}}-\rho_{i k_{2}}=D_{l j}\left(\frac{\sin \beta_{j k_{1}}}{\sin \beta_{k_{1}}}-\frac{\sin \beta_{j k_{2}}}{\sin \beta_{k_{2}}}\right)
$$

we obtain

$$
D_{l j}=\Delta \rho_{l} \frac{\sqrt{\left(1-\cos ^{2} \beta_{k_{1}}\right)\left(1-\cos ^{2} \beta_{h_{z}}\right)}}{\sqrt{\left(1-\cos ^{2} \beta_{k_{2}}\right)\left(1-\cos ^{2} \beta_{j k_{1}}\right)-\sqrt{\left(1-\cos ^{2} \beta_{k_{1}}\right)\left(1-\cos ^{2} \beta_{j k_{2}}\right)}}},
$$

where $\quad ; \beta_{k_{1}}=l_{i k_{1}} l_{j k_{1}}+m_{i k_{1}} m_{j k_{1}}+n_{i k_{1}} n_{j k_{1}}$ etc.

Cases are possible when the components of the observation point-observation point vector are included directly in equalization of space triangulation as "measured" or "fixed" values. 
The value of these components may be obtained, for example, by: processing geodetic measurements, made on the Earth's surface (the basis of space triangulation), or from a previously adjusted space triangulation network.

The relationship of these "measured" values - the orienting angles of chords $\Lambda_{1 j}$ and $\Phi_{1 j}$ or of the length of chords $D_{i j}$ to the unknowns of space triangulation may be represented by expressions similar to Expressions (15.2) and (15.3)

$$
\begin{gathered}
\Lambda_{i j}=\operatorname{arctg} \frac{\Delta Y_{i j}}{\Delta X_{i j}}, \\
\Phi_{i j}=\operatorname{arctg} \frac{\Delta Z_{i j}}{\sqrt{\Delta X_{i j}+\Delta Y_{i j}^{2}}}, \\
D_{i j}=\sqrt{\Delta X_{i j}^{2}+\Delta Y_{i j}^{2}+\Delta Z_{i j}^{2}} .
\end{gathered}
$$

Similar to the manner in which the measured bearings of ordinary triangulation form triangles, permitting sequential calculation of the coordinates of triangulation points and forming the triangulation network, the combinations of measured values and elements of space triangulation form figures which facilitate calculation of the coordinates of observation points. Space triangulation may thus be considered as a combination of separate figures.

We shall consider in detail below the principal types of figures of space triangulation. In this case only elementary figures will be considered, 1.e., figures which contain a minimum number of measurements in input data, required for calculation of the position of the observation point. 
17. Elementary Figures of Space Triangulation, Constructed

From Photographic Observations of AES

Three-Dimensional Angular Intersections

If two AES positions $(k=1,2)$. were observed from two initial ( $1=I, I I)$ and one calculated ( $J=I I I)$ observation point (FIgure 17), calculation of the coordinates of the point being determined may be accomplished by sequential solution of two direct intersections and one inverse angular intersection.

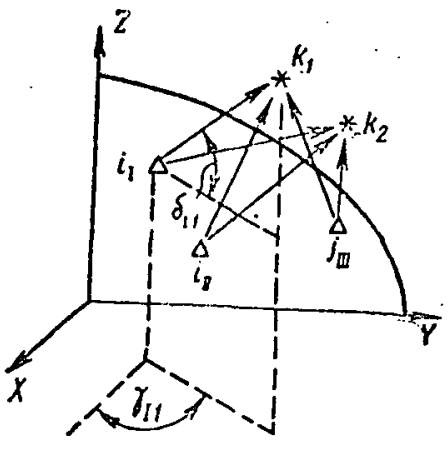

Figure 17.
In the general case, the result of errors in observations, the bearings $\bar{\rho}_{i k}$ from the observation points toward the AES positions do not intersect. To derive the intersection formulas, we shall assume that the bearings do intersect, 1.e., the conditions of coplanarity are fulfilled

$$
\begin{aligned}
& \left(\bar{\rho}_{I 1} \times \bar{\rho}_{I 1}\right) \bar{D}_{I I I}=0 \\
& \left(\bar{\rho}_{12} \times \bar{\rho}_{I 2}\right) \bar{D}_{1 I I}=0 \\
& \left(\bar{\rho}_{I I 1} \times \bar{\rho}_{I I 2}\right) \bar{\rho}_{12}=0 .
\end{aligned}
$$

The method of satisfying these conditions will be considered in the section on adjusting calculations.

Based on the solution sequence, let us determine the position of the satelifte $k=1$. For this, we write the condition of the sum of three vectors

$$
\bar{D}_{111}+\bar{\rho}_{11}+\bar{\rho}_{111}=0
$$


or in projections on coordinate axes

$$
\left\{\begin{array}{c}
\left(X_{I}-X_{I I}\right)+\rho_{I 1} \cos \gamma_{I 1} \cos \delta_{I 1}-\rho_{I I 1} \cos \gamma_{I 1} \cos \delta_{I I 1}=0 \\
\left(Y_{I}-\dot{Y}_{I I}\right)+\rho_{I 1} \sin \gamma_{I 1} \cos \delta_{I 1}-\rho_{I I 1} \sin \gamma_{I 11} \cos \delta_{I I 1}=0 \\
\left(Z_{I}-Z_{I I}\right)+\rho_{I 1} \sin \delta_{I 1}-\rho_{I I} \sin \delta_{I I 1}=0
\end{array}\right.
$$

From the solution of any two equations (for example, the first and second), we calculate the distances from the observation points to the satellite

$$
\begin{gathered}
\rho_{11}=\left[\left(X_{11}-X_{1}\right) \sin \gamma_{11}-\left(Y_{11}-Y_{1}\right) \cos \gamma_{111}\right] \sec \delta_{11}^{\prime} \operatorname{cosec}\left(\gamma_{11}-\gamma_{11}\right) \\
\rho_{11}=\left[\left(X_{11}-X_{1}\right) \sin \gamma_{11}-\left(Y_{11}-Y_{1}\right) \cos \gamma_{11}\right] \times \\
\times \sec \delta_{11} \operatorname{cosec}\left(\gamma_{11}-\gamma_{11}\right) .
\end{gathered}
$$

The third equation of (17.3) controls the calculation.

We obtain formulas directly from Equations (17.3) to calculate the satellite coordinates.

$$
\begin{gathered}
x_{1}=X_{1}+\rho_{11} \cos \gamma_{11} \cos \delta_{11}=X_{11}+\rho_{11} \cos \gamma_{111} \cos \delta_{111} ; \\
y_{1}=Y_{1}+\rho_{11} \sin \gamma_{11} \cos \delta_{1}=Y_{11}+\rho_{I 11} \sin \gamma_{111} \cos \delta_{11} ; \\
z_{1}=Z_{1}+\rho_{11} \sin \delta_{11}=Z_{11}+\rho_{11} \sin \delta_{11} .
\end{gathered}
$$

Instead of calculating the values of $\rho_{I^{1}}$ and $\rho_{I I^{1}}$, we may, by eliminating them from Equations (17.5), obtain formulas for calculating the satellite coordinates in the form

$$
\begin{aligned}
& x_{1}=\frac{Y_{\mathrm{II}}-Y_{\mathrm{I}}+X_{1} \operatorname{tg} \gamma_{11}-X_{\mathrm{II}} \operatorname{tg} \gamma_{\mathrm{II} 1}}{\operatorname{tg} \gamma_{\mathrm{I} 1}-\operatorname{tg} \gamma_{\mathrm{II} 1}} ; \\
& y_{1}=\frac{X_{I I}-X_{I}+Y_{I} \operatorname{ctg} \gamma_{I 1}-Y_{I I} \operatorname{ctg} \gamma_{I I 1}}{\operatorname{ctg} \eta_{11}-\operatorname{ctg} \gamma_{I 1}} ; \\
& z_{1}=Z_{1}+\Delta x_{11} \sec \gamma_{I 1} \operatorname{tg} \delta_{I 1}=: Z_{1}+\Delta y_{11} \operatorname{cosec} \gamma_{11} \operatorname{tg} \delta_{11} ; \\
& z_{1}=Z_{\mathrm{II}}+\Delta x_{1 I I} \sec \gamma_{11} \operatorname{tg} \delta_{1 \mathrm{II}}=Z_{I I}+\Delta Y_{1 \mathrm{II}} \operatorname{coces} \gamma_{\mathrm{II} I} \operatorname{tg} \delta_{1 I} \text {. }
\end{aligned}
$$


The last formula is for control of the calculations. It is obvious that the first two formulas of Expression (17.6) are obtained as formulas of plane intersection in projection of the three-dimensional construction on the equatorial plane. If the angle of intersection in the equatorial plane $\left(\gamma_{I I^{1}}-\gamma_{I^{1}}\right)$ is too acute or obtuse, we may obtain similar formulas from (17.5) by projection of the entire structure onto planes XOZ or YOZ.

The coordinates for the second position of the satellite are calculated in a similar manner, and for this, it is sufficient to replace subscript 1 by 2 in Formulas (17.5)-(17.6). From the calculated coordinates of two satellite positions, after substitution of subscripts I and II by $I$ and 2 , and also $I$ by II in Formulas $(17.5)-(17.6)$, we obtain the coordinates of the observation point being determined ( $\left.\mathrm{X}_{I I I}, \mathrm{Y}_{\text {III }}, \mathrm{Z}_{I I I}\right)$.

The time of each observation is assumed to be known in the construction considered. Of special interest is the special case of a construction, when the flashes of an active satellite of the GEOS type are observed, and the time of flashes are unknown with the required accuracy and, consequently, the angle $\gamma$ for the moment of the flash is unknown. In order to use such observations for space triangulation purposes, it is necessary to either fix the time of star exposures, which is possible when the cameras are operating in a fixed mode, or to calculate the right ascensions of the flashes at the moments their images are obtained, for which the cameras should operate in the star-tracking mode (see Section 14). In the first case, the calculations do not differ in any way from those considered above. In the second case, the moments of the light flashes must first be calculated. For this, the first two equations of (17.1) may be used. After simple transformation of the first equation of (17.1), we have 


$$
a \cos S_{1}+b \sin S_{1}+c=0,
$$

where

$$
\begin{gathered}
a=\operatorname{tg} \delta_{11} \sin \left(\Lambda_{111}-\alpha_{111}\right)-\operatorname{tg} \delta_{111} \sin \left(\Lambda_{11}-\alpha_{11}\right), \\
b=\operatorname{tg} \delta_{11} \cos \left(\Lambda_{111}-\alpha_{111}\right)-\operatorname{tg} \delta_{111} \cos \left(\Lambda_{111}-\alpha_{I 1}\right), \\
c=\sin \left(\alpha_{111}-\alpha_{11}\right) \operatorname{tg} \Phi_{111}
\end{gathered}
$$

From the solution of Equation (17.7), we calculate the value 154 of $S_{1}$.

$$
S_{1}=-\arcsin \left(\frac{c}{b} \cos \varphi\right)-q,
$$

where

$$
\varphi=\operatorname{arctg} \frac{a}{b}
$$

The time of observation of the second AES position, $S_{2}$, is calculated in a similar manner from the second equation of (17.1). After this, the coordinates of two positions of the satellite and of the point being determined are calculated in sequence by the method of three-dimensional angular intersections.

It is easy to see that each position of the AES must be observed from not less than three observation points - two initial and one calculated - during transfer of the coordinates with intermediate calculation of the time of the flashes. Consequently, the construction of the elementary figure of space triangulation shown in Figure 15 is compulsory in this case, unlike observations with fixed time, when, as will be shown below, the coordinate transfer is also possible through observation of the AES positions from two points. 


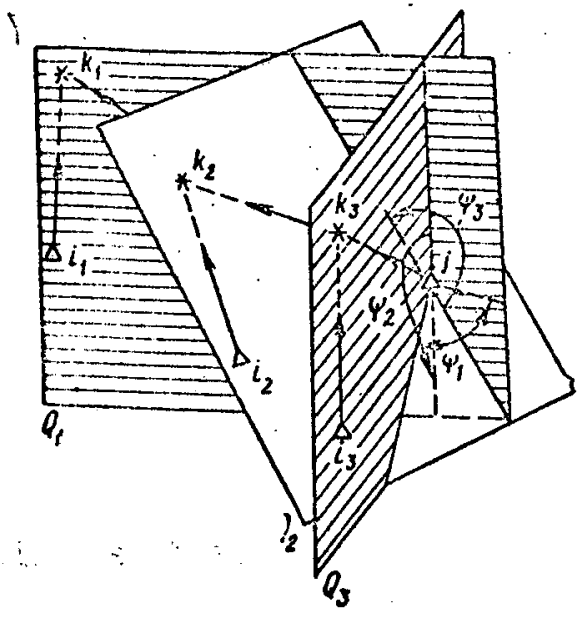

Figure 18.

Let us note the possibility of calculating the coordinates of the observation point being determined during observation of AES flashes at this point by fixed cameras in the absence of a time bureau. In this case, the measured values should be assumed to be only angles $\delta$ and, in order to determine the point, it is necessary to observe four AES positions from 1t, whose coordinates are calculated by observations from initial observation points. The formulas required for calculating the coordinates may be obtalned from the four equations of (15.2), compiled for measured angles $\delta$.

\section{Intersection of Synchronization Planes}

Depending on the mutual distance of observation points, the nature of satelilte trajectory, visibility conditions and a number of other reasons, it may be that each position of the satelite Is observed simultaneously from two points only: the calculated and the initial ones. Such a construction is shown in Figure 18.

The previously consldered method of obtaining the coordinates of the observation point being determined by three-dimensional angluar intersections cannot be used in this case. Let us write the Equations (16.2) for three synchronization planes, formed in a single construction plot,*

$$
\begin{aligned}
& \frac{\left(\bar{\rho}_{\mathrm{II} 1} \times \bar{\rho}_{\mathrm{III} 1}\right) \cdot \bar{D}_{\mathrm{ILIII}}}{\left|\bar{\rho}_{\mathrm{II} 1} \times \bar{\rho}_{\mathrm{HI} 1}\right|}=0, \quad \frac{\left(\bar{\rho}_{\mathrm{II} 2} \times \bar{\rho}_{\mathrm{III} 2}\right) \bar{D}_{\mathrm{IIIII}}}{\left|\bar{\rho}_{\mathrm{II} 2} \times \bar{\rho}_{\mathrm{HI} 2}\right|}=0, \\
& \frac{\left(\bar{\rho}_{\mathrm{I}} \times \bar{\rho}_{\mathrm{III}}\right) D_{\mathrm{IIII}}}{\left|\bar{\rho}_{\mathrm{I}} \times \bar{\rho}_{\mathrm{III}}\right|}=0 \text {, }
\end{aligned}
$$

*The Initial observation points are denoted in the equations by subscripts I and II, the calculated observation point - by III, and the AES positions - by $1,2,3$. 
or in coordinate form

$$
\begin{aligned}
& \ddot{A}_{1} \dot{X}_{\mathrm{III}}+B_{1} Y_{\mathrm{III}}+C_{1} Z_{\mathrm{III}}+W_{1}=0 \\
& A_{2} X_{I I I}+B_{2} Y_{\mathrm{III}+}+C_{2} Z_{\mathrm{III}+W_{2}}=0 \\
& A_{3} X_{\mathrm{XII}}+B_{3} Y_{\mathrm{III}}+C_{3} Z_{\mathrm{III}+}+W_{3}=0
\end{aligned}
$$

where

$$
\begin{aligned}
& W_{v}=-A_{v} X_{l}-B_{v} Y_{i}-C_{v} Z_{l} \\
& v=1,2,3 \\
& i=\text { II at } v=1 \text {, } \\
& i=\mathrm{T} \text { at } v=2,3 \text {. }
\end{aligned}
$$

We obtain the coordinates of the observation point to be determined from solution of the three Equations (17.9), in which there are three unknowns.

Angular Intersection of Chords

If two positions each of the AES are observed from the ends of each bearing, connecting the initial and calculated points

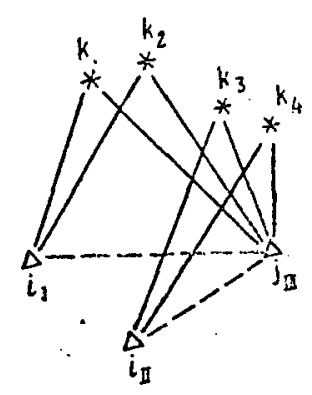

Figure 19.

(F1gure 19), to solve the problem we may use the bearings of the chords between the initial observation points $i_{I}$ and $i_{I I}$ and the calculated observation point $J_{\text {III }}$. To calculate the bearing of each of the chords (orienting angles), connecting two observation points (for example, $i_{I}$ and $1_{\text {III }}$ ), we write the conditional equations of the synchronization planes resulting in this construction in the form.

$$
\begin{aligned}
& \frac{\left(\bar{\rho}_{11} \times \bar{\rho}_{I I 1}\right) \bar{D}_{1 I I I}}{\left|\left(\bar{\rho}_{11} \times \bar{\rho}_{I I 1}\right) \bar{D}_{I I I}\right|}=0, \\
& \frac{\left(\bar{\rho}_{12} \times \bar{\rho}_{I I I}\right) \bar{D}_{I I I I}}{\left|\left(\bar{\rho}_{12} \times \bar{\rho}_{I I I}\right) \bar{D}_{I I I I}\right|}=0
\end{aligned}
$$


or

$$
\begin{aligned}
& A_{1} L_{\text {IIII }}+B_{1} M_{\text {I III }}+C_{1} N_{\text {I III }}=0 \\
& A_{2} L_{\text {IIII }}+B_{2} M_{\text {I III }+}+C_{2} N_{\text {I III }}=0 .
\end{aligned}
$$

Taking the fact into account that the direction cosines $\mathrm{L}$, $M$ and $N$ are related by a single condition $\left(L^{2}+M^{2}+N^{2}=1\right)$, it is sufficient to find two of them, or two of their ratios* from the solution of Equations (17.10). Having divided Equations (17.10) by one of the unknowns, known to be different from zero, for example, by $\mathrm{N}$, we obtain

$$
\begin{aligned}
& A_{1}\left(\frac{L}{N}\right)_{\text {I III }}+B_{1}\left(\frac{M I}{N}\right)_{I I I I}+C_{1}=0 \\
& A_{2}\left(\frac{L}{N}\right)_{\text {I III }}+B_{2}\left(\frac{M}{N}\right)_{I I I I}+C_{2}=0 .
\end{aligned}
$$

We find the ratios of the direction cosines from the solution 156 of Equations (17.11) and, taking the fact into account that

$$
\begin{gathered}
L=\cos \Phi \cos \Lambda \\
M=\cos \Phi \sin \Lambda \\
N=\sin \Phi
\end{gathered}
$$

we calculate the orienting angles of the chord

$$
\begin{gathered}
\Lambda_{\mathrm{IIII}}=\operatorname{arctg}\left[\left(\frac{M}{N}\right)_{I I I I}:\left(\frac{L}{N}\right)_{I I I I}\right] \\
\Phi_{I I I I}=\operatorname{arctg}\left[\left(\frac{L}{N}\right)_{I I I I}^{2}+\left(\frac{M}{N}\right)_{I I I I}^{2}\right]^{-1 / 2}
\end{gathered}
$$

Angles $\Lambda_{\text {II III }}$ and $\Phi_{\text {II.III, which characterize the direction }}$ of the chord ${ }^{1}$ II $-J_{I I I}$ are calculated in a similar manner.

* Instead of the ratio of the direction cosines, in a number of papers the ratios of coordinate increments are calculated, which is the same thing. 
Expressions (17.11) and (17.12) acquire a somewhat different form when divided by $I_{I}$ III $\neq 0$ or $M_{I}$ III $\neq 0$.

The coordinates of observation point $j_{\text {III }}$ being determined may now be calculated from the formulas of three-dimensional intersection (17.3) - (17.6), substituting in them the values $\gamma$ and $\delta$, respectively, for $\Lambda$ and $\Phi$.

18. Elementary Figures of Space Triangulation, Constructed from Combinations of Photographic, Doppler and Laser Observations

The elementary figures for determination of the position of observation points, formed by combined observations, are very diverse and may be both simpler and more complex than the figures constructed only from photographic observations.

If the elementary figures of space triangulation, considered in the preceding section, provided only synchronous photographic observations, they may also contain bearings obtained from asynchronous photographs when combining photographic observations with measured distances or the differences in the distances of the figure.

Let us consider individually the elementary figures for determination of AES positions, and then - the observation points, since it would be very cumbersome to present all possible combinations of simultaneous determination of AES and observation point positions, which would only make it difficult to solve the problem. 


\section{Linear-Angular Intersections in Space}

The simplest will be the elementary figure formed if the direction (angles $\gamma$ and $\delta$ ) and length ( $\rho$ ) of the observation point- satellite vector are determined (Figure 20 ).

The coordinates of AES position may be obtained from the formulas of polar intersection in space:

$$
\begin{gathered}
x_{k}=X_{i}+\rho_{i k} \cos \delta_{l k} \cos \gamma_{l k}, \\
y_{k}=Y_{l}+\rho_{t k} \cos \delta_{i k} \sin \gamma_{i k}, \\
z_{k}=Z_{l}+\rho_{i k} \sin \delta_{l k} .
\end{gathered}
$$

On the other hand, if the position of the AES is known, we obtain the coordinates of the observation point from the same formulas.

A modification of the above-considered elementary figure for determination of AES position is the variant where the direction is measured from one of the observation points $\left(i_{1}\right)$ and the distance to the same position of the AES ( $k$ ) is measured from the other point $\left(1_{2}\right)$ (Figure 21 ).

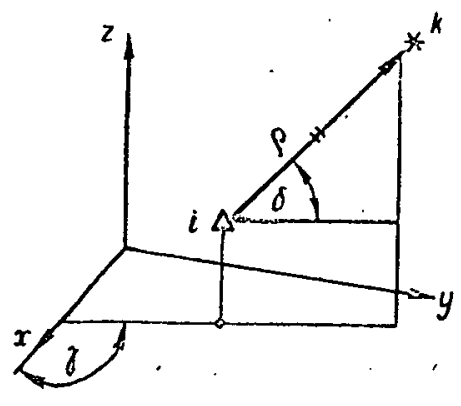

Figure 20.

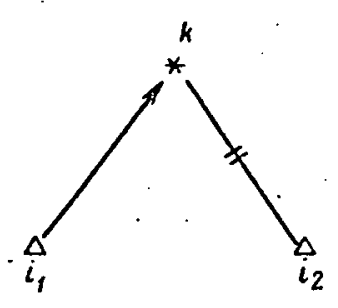

F1gure 21.

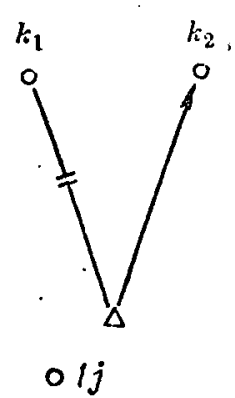

Figure 22.

1 - known position of AES. 
In this case, to calculate the coordinates of the AES, we may compile the following system of equations:

$$
\begin{gathered}
x_{k}-X_{i_{1}}+\rho_{i_{1} k} l_{i_{k} k}=0, \\
y_{k}-Y_{i_{1}}+\rho_{i_{k} k} m_{i_{1} k}=0, \\
z_{k}-Z_{i_{1}}+\rho_{i_{k} k} n_{i_{1} k}=0, \\
\left(x_{k}-X_{i_{2}}\right)^{2}+\left(y_{k}-Y_{i_{3}}\right)^{2}+\left(z_{k}-Z_{i_{z}}\right)^{2}=\rho_{i_{2} k}^{2} .
\end{gathered}
$$

The unknown distance $\rho_{1_{1} k}$ is determined from solution of this system of equations, along with the coordinates of the AES position. If the direction toward one unknown position of the AES and the distance to another is measured from the observation point being determined, the elementary figure (Figure 22) permits calculation of the position of the observation point by linearangular intersection by resolving the system of equations similar to the system of (18.2), where the subscripts $1_{1}, 1_{2}$ and $k$ should be replaced, respectively, by $k_{1}, k_{2}$ and $j$.

Finally, if the length and direction of the chord connecting two ground points 1 and $f$ are calculated, and the position of one ( $i$ ) is known, the coordinates of point $j$ are calculated from the formulas of linear-angular polar intersection:

$$
\begin{gathered}
X_{i}=X_{i}+D_{i j} \cos \Phi_{i j} \cos \Lambda_{i j}, \\
Y_{i}=Y_{i}+D_{i j} \cos \Phi_{i j} \sin \Lambda_{i j}, \\
Z_{j}=Z_{i}+D_{i j} \sin \Phi_{i j} .
\end{gathered}
$$

Linear Intersection in Space

Let us consider the case of determining the position of an AES, when the directions from the observation points to the AES are not measured, but three distances to the position of the AES are measured (Figure 23). Then, obviously, the position of the 


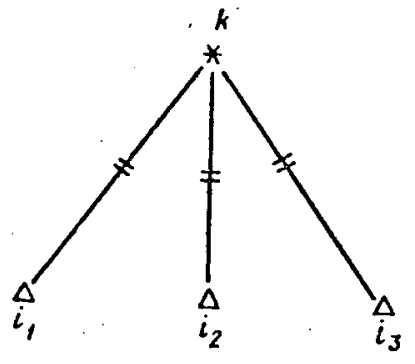

Figure 23.

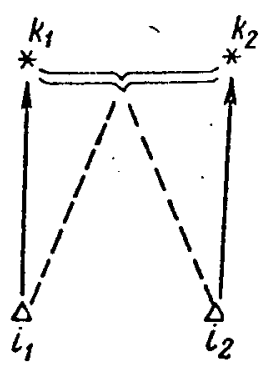

Figure 24.

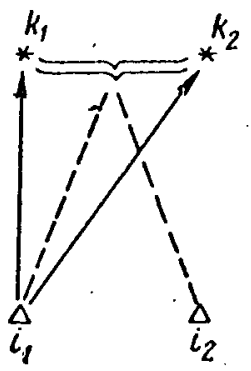

Figure 25.

AES is determined by linear intersection and to calculate the coordinates of the AES we will have a system of three quadratic equations

$$
\begin{aligned}
& \left(x_{k}-X_{i_{1}}\right)^{2}+\left(y_{k}-Y_{i_{1}}\right)^{2}+\left(z_{k}-Z_{i_{1}}\right)^{2}=\rho_{i_{1} / k}^{2}, \\
& \left(x_{k}-X_{i_{3}}\right)^{2}+\left(y_{k}-Y_{i_{2}}\right)^{2}+\left(z_{k}-Z_{i_{2}}\right)^{2}=\rho_{i_{2}, k}^{2} \text {, } \\
& \left(x_{k}-X_{i_{b}}\right)^{2}+\left(y_{k}-Y_{i_{b}}\right)^{2}+\left(z_{k}-Z_{i_{s}}\right)^{2}=\rho_{i_{3} k}^{2} \text {. }
\end{aligned}
$$

A completely similar figure may be used to obtain the coordinates of the observation point, if the distances to the three known positions of the AES are measured from it.

Figures for Determining the Position of AES from Directions and the Differences in Distances

When combining the directions measured from the observation points with the differences in distances to the two AES positions, the elementary figures should permit simultaneous calculation of the coordinates of both unknown positions of the AES.

Two types of such elementary figures containing two each bearings toward the AES positions and two differences in distances from two observation points to the AES positions are essentially possible, differing by the fact that in the first case (Figure 24) the directions are measured from each of two 
observation points to one of the AES positions, and in the second case (Figure 25), the directions are measured only from one observation point to both AES positions.

The coordinates of the AES positions in the first figure determine both the coordinates of the ends of vectors $\bar{p}_{1_{1} k_{1}}$ and $\bar{\rho}_{1_{2} k_{2}}$ from Formulas (18.1). In this case, the unknown values of the lengths of vectors $\rho_{i_{1} k_{1}}$ and $\rho_{i_{2} k_{2}}$ are determined from the solution of two quadratic equations, compiled on the basis of the cosine law

$$
\begin{gathered}
\left(\rho_{22}+\Delta \rho_{2}\right)^{2}==\rho_{11}^{2}+D_{i_{1} i_{2}}^{2}-2 \rho_{11} D_{i_{i} i_{2}}\left(L_{i_{i} i_{2}} l_{11}+-M_{i_{1} i_{2}} m_{11}+N_{i_{1} i_{2}} n_{11}\right) \\
\left(\rho_{11}+\Delta \rho_{1}\right)^{2}=\rho_{22}^{2}+D_{i_{1}}^{2} i_{2}-2 \rho_{22} D_{i_{1} i_{2}}\left(L_{i_{1} i_{2}} l_{22}+M_{i_{1} i_{2}} m_{22}+N_{\left.i_{1} i_{2} n_{22}\right)}\right) .
\end{gathered}
$$

Similarly, in the second figure the coordinates of AES positions are calculated from the same formulas of (18.1) for vectors $\bar{\rho}_{1_{1} k_{1}}$ and $\bar{\rho}_{i_{1} k_{2}}$. The unknown values of the lengths of vectors $\rho_{1_{1} k_{1}}$ and $\rho_{1_{1} k_{2}}$, jointly with the distance $\rho_{1_{2} k_{2}}$ - which Is unknown but unnecessary for solution of the problem - are calculated from the same equations of (18.5) with consideration of $\rho_{1_{1} k_{2}}=\rho_{1_{1} k}+\Delta \rho_{1}$.

Cases are possible when photographic observations are combined with the differences in distances, measured from the observation points, to two AES positions, of which only one is an unknown (the other is calculated, for example, with the ald of synchronous photographic observations alone).

Such combinations of measurements make it possible to consider the elementary figures for determining on position of the AES, which should be established by three measured values. Three types of such elementary figures are possible: 


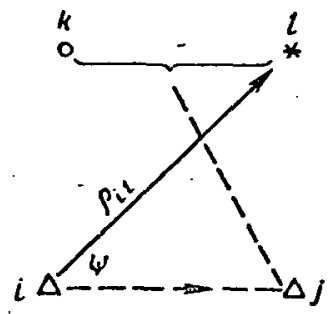

Figure 26.

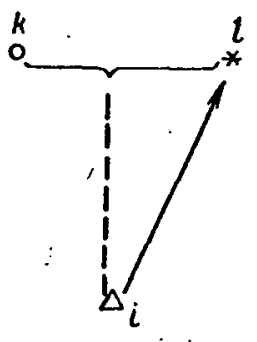

Figure 27.

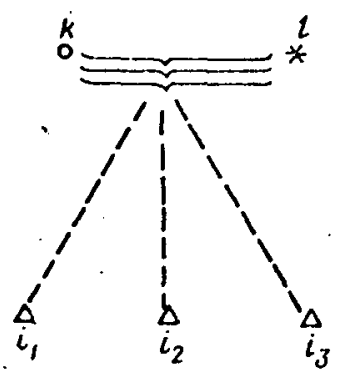

F1gure 28.

- the direction to one AES position is measured from one observation point and the difference in distances to this and st1ll another unknown position of the AES - from another observation point (Figure 26). The problem is solved similarly, if the direction and difference in distances are measured from a single observation point (Figure 27);

- the differences in distances to two AES positions are measured from three observation points, one of which is unknown (Figure 28).

Let us consider the figure presented in Figure 26. The coordinates of the $\tau^{\text {th }}$ position of the AES may be obtained from Formulas (18.1), if distance $\rho_{12}$ is calculated. Let us consider triangles ifl and ik $l$ to calculate this distance. The distance $\rho_{k i}, \rho_{i j}$ and $\rho_{k j}$ may be calculated by the unknown coordinates of the $k^{\text {th }}$ position of the AES and points $i$ and $j$, and we find distance $\rho_{j l}$ by using the measured difference in distances and distance $\rho_{\mathrm{kj}}$. We must now find distance $\rho_{12}$. For this, we apply the cosine law to triangle ij $z$. The cosine of angle $z_{i j}(\psi)$ may be calculated, because the direction cosines of bearing 12 are known (from measured angles $\gamma$ and $\delta$ ), and direction cosines of 
bearing if are calculated from the coordinates. Solving the quadratic equation and discarding the redundant solution, we will have

$$
\begin{gathered}
\rho_{i l}=\rho_{i j} \cos \psi+\sqrt{\rho_{i j}^{3} \cos ^{3} \psi-\rho_{i j}^{3}+\left(\rho_{i k}^{2}+-\Delta \rho\right)^{2}} \\
\cos \psi=l_{i j} l_{l l}+m_{l i} m_{l l}+n_{l j} n_{l l} .
\end{gathered}
$$

In the case when the difference in distances and bearing are measured from a single observation point (see Figure 27), calculation of distance $\rho_{1 l}$ is simplified

$$
\rho_{i l}=\rho_{l k}+\Delta \rho \text {. }
$$

In the case when the difference in distances to two positions of the AES are measured from three observation points (see Figure 29), to calculate the coordinates of the point we may complie a system of three quadratic equations of the type

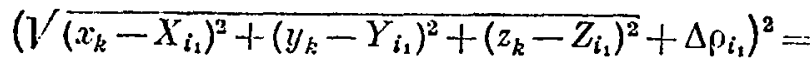

$$
\begin{aligned}
& =\left(x_{l}-X_{i_{1}}\right)^{2}+\left(y_{l}-Y_{i_{1}}\right)^{2}+\left(z_{l}-Z_{i_{1}}\right)^{2} \text {. }
\end{aligned}
$$

Similar equations may be written for observations from observation points $i_{2}$ and $i_{3}$.

Figures for Determining the Position of the Observation Point from the Bearings and Differences in Distances

If we consider that the AES positions are known, combining the measured directions and differences in distances from the observation point being determined to the known positions of the AES produces two types of elementary figures. 


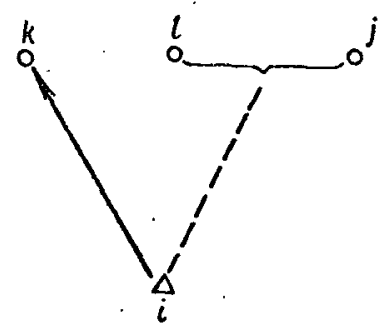

Figure 29.

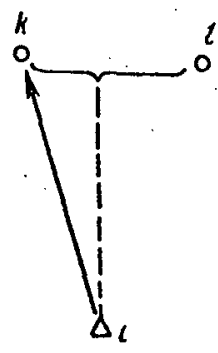

Figure 30.

In the first case, the difference in distances to two AES positions and the bearing toward the third position are obtained from the observation point being determined (Figure 29). In the second case, the bearing is measured to one of two AES positions, the difference in distances to which has been calculated (Figure 30).

The position of the observation point is calculated from the first elementary figure by Formulas (18.1). To find distance $\rho_{i k}$, two quadratic equations should be solved which are derived from the solution of triangles $1 \mathrm{kl}$ and $1 \mathrm{kj}$ from the cosine formula. In this case, the distance $\rho_{12}$ is calculated simultaneously with distance $\rho_{1 k}$. Distances $\rho_{k l}$ and $\rho_{k j}$ are calculated from the known coordinates. The cosines of angles $1 \mathrm{kl}$ and $1 \mathrm{kj}$ are found in the product of the direction cosines of the bearings of their generatrices. For bearing ik, the direction cosines $z_{k i}, m_{k i}$ and $n_{k i}$ are found from measured angles $\gamma_{i k}$ and $\delta_{i k}$, taking the fact into account that $\tau_{k 1}=-\tau_{1 k}, m_{k i}=-m_{i k}$ and $n_{k i}=-n_{i k}$

The direction cosines are calculated from the coordinates for bearlings $\mathrm{kl}$ and $\mathrm{kj}$. The distance $\rho_{1 \mathrm{j}}=\rho_{12}+\Delta \rho_{1}$, where $\Delta \rho_{1}$ is the measured difference in distances. We have: 


$$
\begin{gathered}
\rho_{i k}^{2}+\rho_{h j}^{2}-2 \rho_{i k} \rho_{k j}\left(l_{k i} l_{k j}+m_{k l} m_{k j}+n_{k i} n_{k j}\right)=\left(\rho_{i l}+\Delta \rho\right)^{2} \\
\rho_{i k}^{2}+\rho_{h l}^{2}-2 \rho_{i k} \rho_{k l}\left(l_{k i} l_{k l}+m_{k i} m_{k l}+n_{k i} n_{k l}\right)=f_{i l}^{2} .
\end{gathered}
$$

In the second elementary figure, the coordinates of the observation point are also calculated from Formulas (18.1), but distance $\rho_{1 k}$ is calculated by the formula

$$
\rho_{t k}=\frac{\rho_{k l}^{2}-\Delta \rho^{2}}{2\left[\Delta \rho+\rho_{k l}\left(l_{k l} l_{k l}+m_{k i} m_{k l}+n_{k i} n_{k l}\right)\right]} .
$$

Let us now consider a case when only the differences in distances are oblserved from the observation point. Then three distances should be measured in the elementary figure - between the two pairs of AES positions (FIgure 31). To find the coordinates of the point in this figure, it is easy to compile a system of three equations

$$
\begin{aligned}
& \rho_{i j}^{2}=\left(\rho_{i k}+\Delta \rho_{1}\right)^{2}, \\
& \rho_{i l}^{2}=\left(\rho_{i m}+\Delta \rho_{2}\right)^{2}, \\
& \rho_{i n}^{2}=\left(\rho_{i p}+\Delta \rho_{3}\right)^{2}
\end{aligned}
$$

or in coordinate form

$$
\begin{aligned}
\left(X_{i}-x_{j}\right)^{2} & +\left(Y_{i}-y_{j}\right)^{2}+\left(Z_{i}-z_{j}\right)^{2}=\left(X_{i}-x_{k}\right)^{2}+\left(Y_{i}-y_{k}\right)^{2}+\left(Z_{i}-z_{k}\right)^{2}+ \\
& +2 \Delta \rho_{1} \sqrt{\left(X_{i}-x_{k}\right)^{2}+\left(Y_{i}-y_{k}\right)^{2}+\left(Z_{i}-z_{k}\right)^{2}}+\Delta \rho_{1}^{2}, \\
\left(X_{i}-x_{l}\right)^{2}+ & -\left(Y_{i}-y_{l}\right)^{2}+\left(Z_{i}-z_{l}\right)^{3}=\left(X_{i}-x_{m}\right)^{2}+\left(Y_{i}-y_{m}\right)^{2}+\left(Z_{i}-z_{n}\right)^{2}+ \\
& +2 \Delta \rho_{2} \sqrt{\left(X_{i}-x_{m}\right)^{2}+\left(Y_{i}-y_{m}\right)^{2}+\left(Z_{i}-z_{m}\right)^{2}}+\Delta \rho_{2}^{2}, \\
-\left(X_{i}-x_{n}\right)^{2} & +\left(Y_{i}-y_{n}\right)^{2}+\left(Z_{i}-z_{n}\right)^{2}=\left(X_{i}-x_{p}\right)^{2}+\left(Y_{i}-y_{p}\right)^{2}+\left(Z_{i}-z_{p}\right)^{2}+ \\
& +2 \Delta \rho_{3} \sqrt{\left(X_{i}-x_{p}\right)^{2}+\left(Y_{i}-y_{p}\right)^{2}+\left(Z_{i}-z_{p}\right)^{2}}+\Delta \rho_{3}^{2},
\end{aligned}
$$

where $\Delta \rho_{1}, \Delta \rho_{2}$ and $\Delta \rho_{3}$ are the measured differences in distances.

A case is possible when points $k$ and $l$ and $m$ and $n$ will coincide; the solution of the problem does not change in this case. 


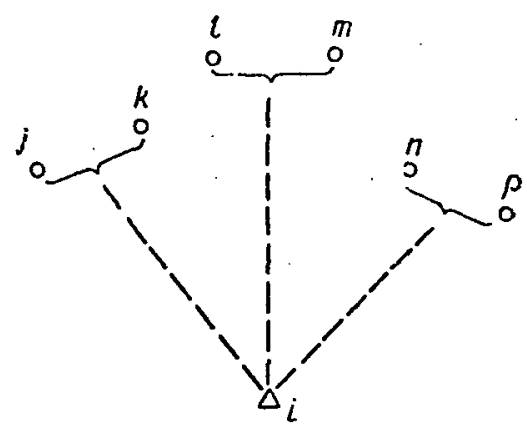

Figure 31.

In conclusion, let us consider an elementary figure which permits the coordinates of the observation point to be calculated from synchronous photographic observations, combined with the measured difference in distances to two AES positions, for one initial observation point (see Figure 16). This figure permits both the direction and length of the observation point-observation point vector to be obtained immediately, 1.e., in the final analysis, it reduces to the case of linear-angular polar intersection mentioned above. Earlier we obtained an expression to calculate the length of this vector from a similar figure (16.12). However, it is necessary in this figure that the positions of the AES form different synchronization planes, whose intersection permits simultaneous calculation of the length and direction of the chord, and at the same time - the position of the observation point.

It is easy to show that in this case we proceed without calculation of the coordinates of AES positions. Actually, to calculate the coordinates of the AES position we can compile four sets of three equations (18.1) from bearings $i k_{1}, i k_{2}, j k_{1}$, and $j k_{2}$. In these 12 equations there will be 13 unknowns - the coordinates $x_{k_{1}}, y_{k_{1}}, z_{k_{1}}, x_{k_{2}}, y_{k_{2}}, z_{k_{2}}, x_{j}, y_{j}$ and $z_{j}$ and four distances $\rho_{1 k_{1}}, \rho_{i k_{2}}, \rho_{j k_{1}}$ and $\rho_{j k_{2}}$. The $13^{\text {th }}$ equation is obtained with the aid of the measured difference in distances

$$
\Delta \rho_{i}=\rho_{i k_{\mathbf{1}}}-\rho_{i k_{\mathbf{2}}} \text {. }
$$

Eliminating the coordinates of AES positions and one of the distances from these 13 equations by simple algebralc transformations, we obtain the following system of six linear algebraic 
equations to calculate the coordinates of observation point $j$ and three distances

$$
\begin{gathered}
-x_{j}+\rho_{i k_{1}} l_{i k_{2}}-\rho_{j k_{2}} l_{j k_{2}}+x_{i}-\Delta \rho l_{i k 2}=0 \\
-y_{j}+\rho_{i k_{2}} m_{i k_{2}}-\rho_{j k_{2}} m_{j k_{2}}+y_{i}-\Delta \rho m_{i k_{2}}=0 \\
-z_{j}+\rho_{i k_{1}} n_{i k_{2}}-\rho_{j k_{2}} n_{j k_{2}}+z_{i}-\Delta \rho n_{i k_{2}}=0 \\
-x_{j}+\rho_{i k_{1}} l_{i k_{1}}-\rho_{j k_{1}} l_{j k_{1}}+x_{i}=0, \\
-y_{j}+\rho_{i k_{1}} m_{i k_{1}}-\rho_{j k_{1}} m_{j k_{1}}+y_{i}=0 \\
-z_{j}+\rho_{i k_{1}} n_{i k_{1}}-\rho_{j k_{1}} n_{j k_{1}}+z_{i}=0
\end{gathered}
$$

Selection of Figures to Construct Space Triangulation

We have considered the simplest elementary figures which permit calculation of the position of the AES or the observation point, 1.e., all three unknown coordinates.

Moreover, it was mentioned earlier that the figures for calculating the AES position are intermediate from the point of view of calculating the position of the observation point, because in themselves the AES positions do not interest us. Moreover, the figures for calculating the position of the observation points may be formed from synchronous observations of AES positions, carried out over a long period of time.

More complex figures than those which we have considered are formed in the process of real observations of AES positions from different observation points. In this case, the number and composition of measured values in these figures may permit immediate calculation of both the AES position as intermediate sighting targets and the position of the point being calculated, such as, for example, in Figure 16. 
In some figures, combination of measurements permits (if the position of the observation points is assumed known) calculation of the position of the AES alone (see Figure 24) or even not to obtain this (Figure 32 ).

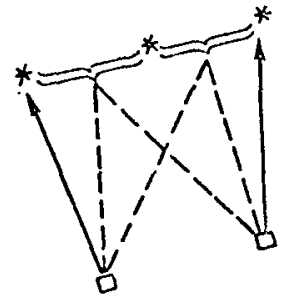

Figure 32.

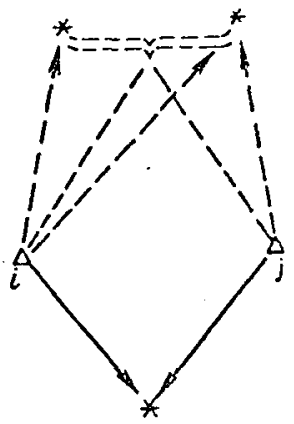

Figure 33.

However, other figures may also arise which contain AES positions and those of a calculated observation point, in which the measured values are greater than necessary for calculation of the AES position, but inadequate for simultaneous calculation of the coordinates of the observation point being determined. Thus, for example, in each of two figures, one of which is shown in Figure 33 by the dashed line, and the other - by the solid lines, there are redundant measurements for determination of AES positions, if the position of both observation points 1 and $j$ are assumed to be known. If observation point $j$ is assumed to be calculated, none of the figures will determine elther the position of the AES nor that of this observation point. Only by considering both figures jointly may the positions of the AES and of observation point $f$ be calculated.

It is clear from the foregoing that figures which also contain redundant information in addition to the measurements determining the AES positions, are required to construct space triangulation for purposes of determining the position of observation points. Therefore, of the entire aggregate of real groups of AES 
observations related among themselves in time, it is necessary to select those figures which contain not less than $3 \mathrm{~s}+1$ measurements, where $s$ is the number of AES positions in the figure, to include space triangulation in a mathematical treatment.

\section{General Principles and Characteristics of Constructing}

\section{Space Triangulation}

Space triangulation is a gemoetric construction, based, as is evident from the figures forming it, on the possibility of determining the mutual position of observation points by observations from them of simultaneous positions of AES. Thus, a satellite in space triangulation is used as the intermediate highaltitude sighting target.

The figures considered in Section 17 determine three principles of construction of space triangulation from photographic observations.

Sequential solutions of pairs of groups of equations (18.1), obtained during synchronous observation of not less than two AES positions from three observation points - two initial and one calculated (Figure 34) - permits the coordinates of the observation points to be calculated by the method of angular intersections in space. This is the first princlpal method of space triangulation construction.

Elimination of the unknown coordinates of the AES position from two pairs of equations (18.1), obtained as a result of synchronous observation of one satellite position from two observation points, leads to the equation of the synchronization plane (16.2'). 


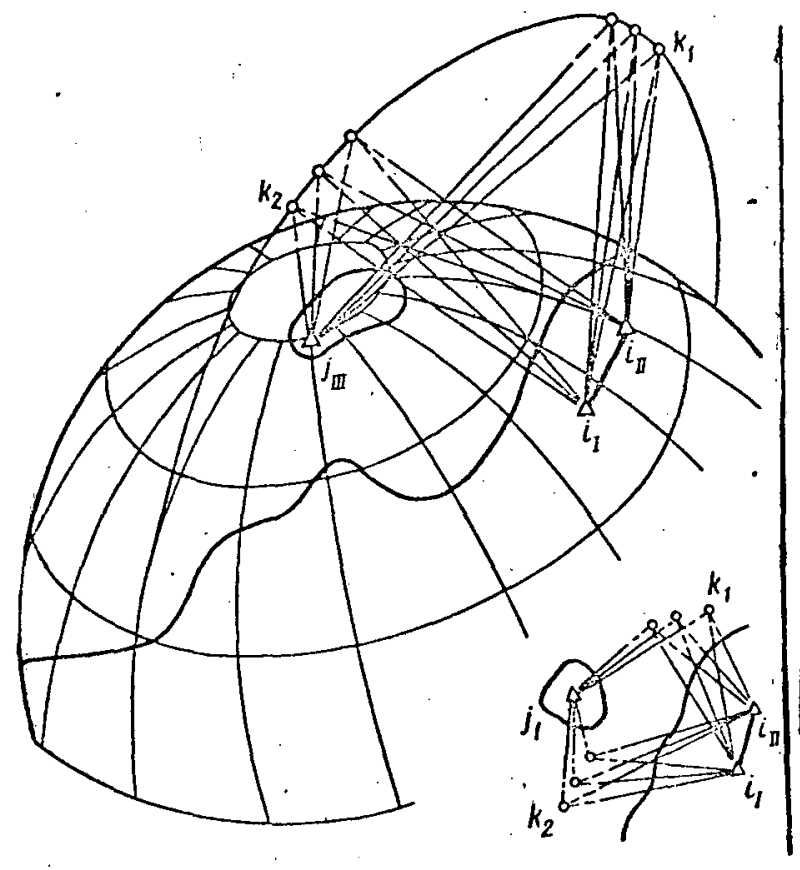

Figure 34.

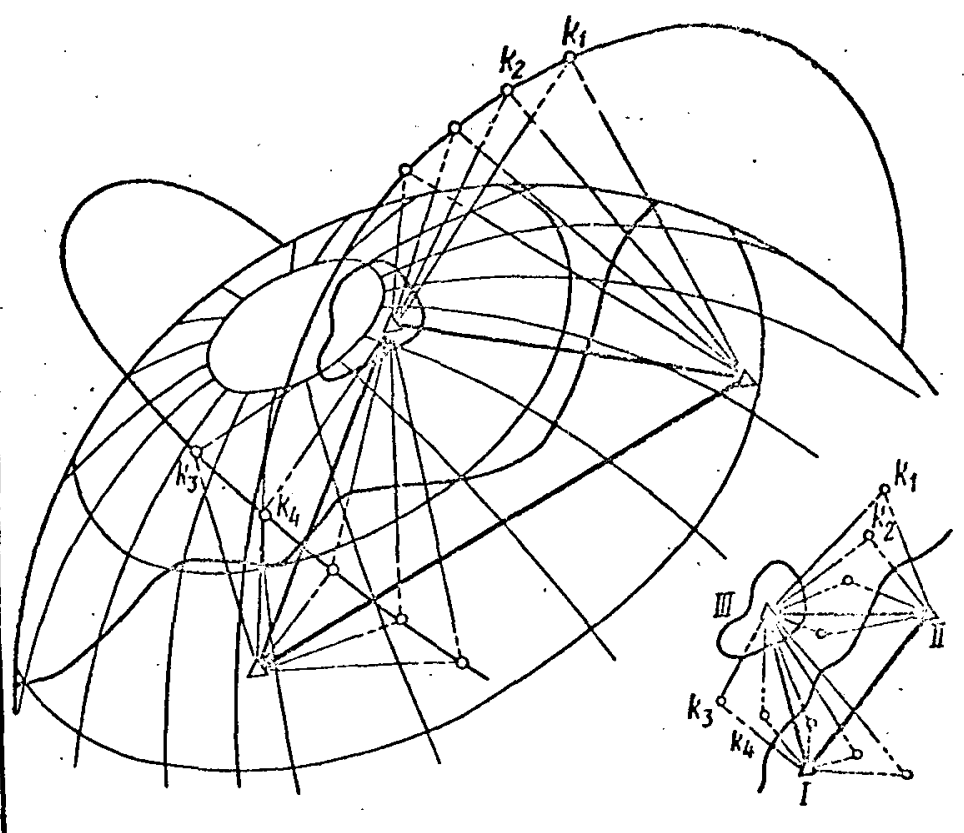

Figure 35.

Joint solution of the equations of not less than three synchronization planes, compiled for not less than two initial and one calculated observation point, permits the position of the latter to be obtained. This principle of construction (see Figure 18) is called the method of planes.

Solution of two Equations (16.2') for each chord between the initial and calculated observation points determines the direction of each of the chords, and the position of the calculated observation point is found at their intersection (Figure 35). This principle of space triangulation construction has been called the method of chords (closing directions).

Depending on the purposes of creating space triangulation, three types of construction may be distinguished: 
1. Separate figures used to determine the position of single points, for example, to tie in a local geodetic system (insular) to a unified geodetic net (mainland), as well as those which are integral parts of more complex types of constructions.

2. Series used to transmit coordinate systems for a considerable distance, or to combine very remote local systems into a unified system with minimum expenditures of labor.

3. Dense nets, used for propagation of a unified coordinate system over a vast territory, or to create a network of observation points of specific density.

These constructions differ in the distribution and number of AES observation points. However, they may all be regarded as a combination of the individual figures forming the space triangulation.

When considering all the problems of construcstion, adjustment, and analysis of the accuracy of space triangulation, two of its characteristic features should be taken into account. First, all measurements in space triangulation are carried out only ifrom ground points and no measurements are carried out from the satellite. The absence of direct observations from the satellite and between ground triangulation points leads to the fact that all measurements in the space triangulation network, unlike ordinary triangulation, are unilateral.

Secondly, space triangulation is distinguished by less reliable determination of individual AES positions than of observation points. This is quite understandable, because each instantaneous position of the AES may be observed only from a specific number of observation points and only at one time. 
A comparatively small number of synchronous observations of the instantaneous position of the AES creates a small number of redundant measurements to determine a given position. And a large number of redundant measurements is accumulated to determine the positions of a comparatively small number of observation points compared to the number of AES positions in a triangulation network constructed over a considerable period of observation time.

As in ordinary triangulation, the problem arises of adjusting measurements in space geodetic networks due to the presence of a considerably larger number of observations than is required for calculating the coordinate points. Adjustment is accomplished by the least squares method. In this case, a set of measured values obtained at the points within a specific period of observations is included in the processing.

The three possible principles of construction of space triangulation which we have outlined, as well as their characterIstics, have revealed the different methods of applying the least squares method to adjustment of space triangulation, which will be elucidated in the following chapter. 
CHAPTER 5

ADJUSTMENT OF SPACE TRIANGULATION

20. Types of Conditions Arising in Space Triangulation Networks

167

When adjusting space triangulation, it is necessary to cope with a number of characteristics which are apparently inherent only to a given type of geodetic network. We recall that the directions between the observation points of space triangulation are determined independently of each other in a unified stellar coordinate system. Therefore, conditions similar to those of sums and azimuths (directional angles) may not arise in such a network. In other words, not one of those conditions which are combined under the general name of angular conditions in an ordinary geodetic network occur in space triangulation.

Polar, base and coordinate conditions are completely retained in space triangulation. But besides them, specific geometric conditions appear in space geodetic networks which have no analogs in plane networks - the conditions of coplanarity of three vectors, plane bundles, and plane sheafs.

A specific type of conditions arising in the figures of space triangulation is considered below.

Conditions of the Coplanarity of Three Vectors

In more general form, the condition of coplanarity of three topocentric vectors $\Delta \bar{R}_{1}, \Delta \bar{R}_{2}$ and $\Delta \bar{R}_{3}$ will be 


$$
\overline{\Delta R}_{1} \overline{\Delta R}_{2} \overline{\Delta R}_{3}=0
$$

or

$$
\left[\bar{r}_{1}-\bar{r}_{2}\right]\left[\bar{r}_{1}-\bar{r}_{3}\right]\left[\bar{r}_{2}-\bar{r}_{3}\right]=0
$$

where $\bar{r}_{1}$ is the value of the radius vectors.

After normalization, we obtain

$$
F=\frac{\left(\bar{r}_{1}-\bar{r}_{2}\right)\left(\bar{r}_{1}-\bar{r}_{3}\right)\left(\bar{r}_{2}-\bar{r}_{3}\right)}{\left|\bar{r}_{1}-\bar{r}_{2}\right|\left|\bar{r}_{1}-\bar{r}_{3}\right|\left|\bar{r}_{2}-\bar{r}_{3}\right|}=0
$$

or

$$
F==\bar{a}_{1.2} \cdot \bar{a}_{1.3} \cdot \bar{a}_{2.3}=0
$$

where

$$
a_{i j}=\frac{\bar{r}_{t}-\bar{r}_{j}}{\left|\bar{r}_{l}-\bar{r}_{j}\right|}
$$

Equation (20.4) 1s the condition of coplanarity of three free vectors. In coordinate form, it has the form

$$
F=\left|\begin{array}{lll}
l_{1.2} & m_{1.2} & n_{1.2} \\
l_{1.3} & m_{1.3} & n_{1.3} \\
l_{2.3} & m_{2.3} & n_{2.3}
\end{array}\right|=0
$$

This condition is the principal one in space triangulation and arises as a result of the synchronism of observation of a single satellite position from two observation points (synchronization planes) or arrangement of three vectors connecting the observation points in a single plane ("the plane of three observation points"). 
Conditions of a Bundle of Planes

The direction of the lines connecting two ground points is clearly determined as the result of intersection of two planes. The condition of the bundle of planes exresses the requirement that all synchronization planes intersect for two observation points on a single chord. Each new plane, added to the first two, will be redundant and, consequently, will lead to a single condition.

Let three planes be defined by their normal vectors $\bar{N}_{1}\left(A_{1} B_{1} C_{1}\right)$, $\overline{\mathrm{N}}_{2}\left(\mathrm{~A}_{2} \mathrm{~B}_{2} \mathrm{C}_{2}\right)$ and $\overline{\mathrm{N}}_{3}\left(\mathrm{~A}_{3} \mathrm{~B}_{3} \mathrm{C}_{3}\right)$. Let us compose a mixed product of these vectors

$$
V=\bar{N}_{1} \bar{N}_{2} \bar{N}_{3}
$$

The mixed product is numerically equal to the volume of a parallelipiped, constructed on vectors $\overline{\mathrm{N}}_{1}, \overline{\mathrm{N}}_{2}$ and $\overline{\mathrm{N}}_{3}$. However, if the planes belong to a single bundle, the parallelipiped degenerates into a line and, consequently, $V=0$. Turning to the coordinate form of a mixed product, we obtain the conditional equation of intersection of three planes on a single line

$$
V=\left|\begin{array}{lll}
A_{1} & B_{1} & C_{1} \\
A_{2} & B_{2} & C_{2} \\
A_{3} & B_{3} & C_{3}
\end{array}\right|=0
$$

Condition of the Sheaf of Planes

Intersection of three planes in space clearly determines a point and each additional plane leads to a single condition, expressing a requirement for intersection of four planes at a single point. 
We know that four planes pass through a single point when the fourth-order determinant, compiled from the coefficients and free terms of planar equations, is equal to zero, i.e.,

$$
\Phi=\left|\begin{array}{llll}
A_{1} & B_{1} & C_{1} & D_{1} \\
A_{2} & B_{2} & C_{2} & D_{2} \\
A_{3} & B_{3} & C_{3} & D_{3} \\
A_{4} & B_{4} & C_{4} & D_{3}
\end{array}\right|=0
$$

Base and Polar Conditions

The conditional base equation arises in the case when two or more initial or measured sides are contained in a space triangulation network provided that there is a direct relationship between these sides through a chain of three-dimensional triangles.

As an example, let us write the base equation for the figure shown in Figure 36 . Solving triangles $1_{1} \mathrm{k} i_{2}$ and $i_{2} \mathrm{kj}$ sequentially,

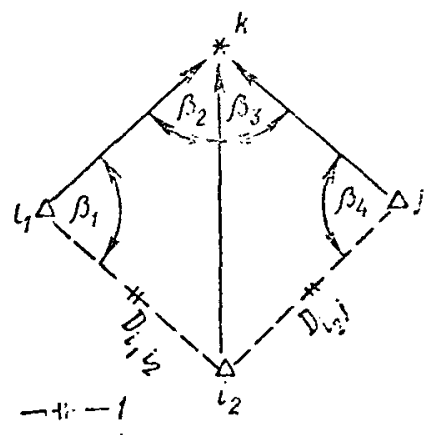

Figure 36.

1 - measured base of chord. It is easy to obtain an expression completely analogous to the conditional base equation for plane figures

$$
F=\frac{\sin \beta_{1} \sin \beta_{3} D_{i_{1} i_{2}}}{\sin \beta_{2} \sin \beta_{1} D_{i_{2} i}}-1=0,
$$

in which $\beta$ are the angles formed by topocentric directions.

A unique feature of the base equation is the condition which arises when the differences from the observation points to the AES positions are measured along with simultaneous photographic observations. 
The polar condition occurs in space triangulation figures in the case when there are closed chains of triangles, which begin and end on the same side. Three-dimensional polar conditional equations are compiled in a similar manner as is done in geodetic networks on a plane.

The form of the conditional polar equation formally precisely gives the expression obtained for the conditional base equation.

Coordinate Conditions

Coordinate conditions occur in the case when there are isolated initial points or systems of them in the space triangulation network. A space triangulation figure is shown in Figure 37, In which the spherical coordinates of all lines connecting the apexes of the given figure are known, where $A$ and $B$ are the initial points, $P$ is the calculated point and $k_{1}$ is the AES positions.

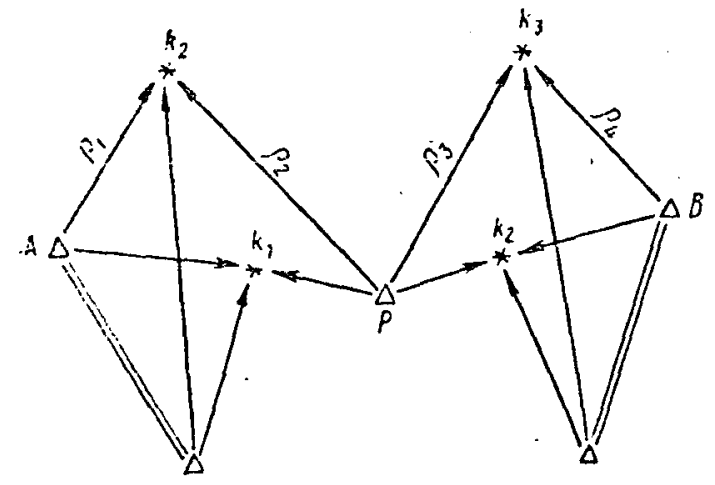

Flgure 37 .

The coordinate condition in vector form for the figure may be written

$$
\bar{\rho}_{1}+\bar{\rho}_{2}+\bar{\rho}_{3}+\overline{\rho_{4}}=\overline{A B} . \quad(20.10)
$$

This condition means that the 170 coordinates calculated from point A along the course line $\mathrm{Ak}_{2} \mathrm{Pk}_{3} \mathrm{~B}$, precisely coincides with the coordinates of initial point $B$. For the coordinate increments the following equations must be satisfied. 


$$
\begin{gathered}
X_{B}-X_{A}=\Delta X=-\cdots \sum_{1}^{n-1} l_{i k} \rho_{i k}, \\
Y_{B}-Y_{A}=\Delta Y=-\cdots=\sum_{1}^{n-1} m_{i k} \rho_{i k}, \\
Z_{B}-Z_{A}=:=\Delta Z=:=\sum_{1}^{n-1} n_{i k} \rho_{i k},
\end{gathered}
$$

where 1 ' is the number of the observation point $(1=1,2, \ldots, n)$ and $k$ is the number of the AES position $(k=1,2, \ldots, n-1)$, from which the conditional equations for the coordinates are easily obtained

$$
\begin{gathered}
\varphi_{x}==\sum_{1}^{n-1} l_{i \leq} \rho_{i k}-\Delta X_{n, 1}=0, \\
\varphi_{y}=\sum_{i}^{n-1} m_{i k} \rho_{i k}-\Delta Y_{n, 1}=0, \\
\varphi_{z}=\div \sum_{1}^{n-1} n_{i k} \rho_{i k}-\Delta Z_{n, 1}=0 .
\end{gathered}
$$

Selection of Conditions

Most conditions arising in ordinary geodetic networks have the property of equivalency or interchangeability, which leads to the selection of the simpler in form or that containing the least number of unknowns compared to other conditions, from several relations linking any group of measured values.

The conditions arising in a space triangulation network also have the properties of equivalency.

The condition of a sheaf of planes, which expresses the requirement that the synchronization planes intersect at the points of AES observations, is more universal from the geometric point of view. Actually, synchronization planes must pass through the initial points (the three terms of the equations of these planes are calculated under this requirement). Moreover, the planes constructed with the ald of measurements from some calculated point, after adjustment, form their own sheaf with the 
center at this point. Thus, after the conditions of sheaves of the planes in a given network have been satisfied, the polar and coordinate conditions should be automatically satisfied. In other words, the condition of the sheaf of planes is equivalent to any other condition, with the exception of the condition of base for directly measured sides.

The conditions of the sheaf of planes and the bundle of planes in turn are equivalent to definition of four and three equations of synchronization planes, respectively ("the planes of three points"). Therefore, any conditions of space triangulation, besides base conditions, may be described by the appropriate number of planar equations.

However, the planar condition requires definition of the coordinates of two points through which this plane passes. Consequently, for conditional planar equations, passing through calculated observation points, it is necessary that the coordinates of the latter be expressed by all the measured values, by means of which these coordinates are calculated, beginning from the initial points. This would lead to an extremely cumbersome and irregular form for each planar condition. In order to use single type and simpler planar conditions during adjustment, the coordinates of the calculated points may be regarded as additional unknowns (parameters).

The preliminary estimate of accuracy is another thing. The values of the free terms of conditional planar equations with additional unknowns depend both on measurement errors and on definition of the preliminary values of the unknowns. It is clear that the free terms of these equations may not be used to analyze the accuracy of the measurement results in terms of 
the residuals discrepancies of the geometric conditions. Conditional equations without additional unknowns must be used to analyze accuracy.

21. Adjustment of Space Triangulation by the Parametric Method (the Method of Direct Measurements)

When adjusting an entire set of measured values of triangulation by the parametric method for each measured value, connecting the position of the observation point to that of the AES, a correction equation is compiled in which correction for the measured value in explicit form is expressed by corrections of unknown coordinates (parameters).

\section{The Correction Equation for Angles $\gamma$ and $\delta$}

The functional relationship of angle $\gamma$ to the geocentric coordinates is defined by Equation (15.2).

After it has been reduced to linear form, we will have

$$
\begin{aligned}
\gamma_{i k}^{\prime}+v_{\gamma}=-\operatorname{arctg}\left(\frac{y_{k}-\dot{Y}_{i}}{x_{k}-X_{l}}\right)_{0}+\frac{\partial y}{\partial x_{k}} d x_{k}+\frac{\partial y}{\partial y_{k}} d y_{k}+ \\
+\frac{\partial y}{\partial X_{l}} d X_{l}+\frac{\partial \gamma}{\partial Y_{l}} d Y_{l} .
\end{aligned}
$$

Let us introduce the notations

$$
\begin{gathered}
d x_{k}=\xi_{k} ; \\
d y_{k}=\eta_{k} ; \\
d X_{i}=\xi_{i} ; \\
d Y_{i}=\eta_{i} ; \\
s_{i k}=\sqrt{\left(x_{k}-X_{i}\right)^{2}+\left(y_{k}-Y_{i}\right)^{2}}=\rho_{i k} \cos \delta_{i k}, \\
l_{\gamma}=\operatorname{arctg}\left(\frac{y_{k}-Y_{i}}{x_{k}-X_{l}}\right)_{0}-\gamma_{i k}^{\prime}
\end{gathered}
$$


It is assumed in Formulas (21.1): $\rho$ is the distance from point 1 to the AES position $k$; $s$ is the projection of this distance on the coordinate plane XOY (the plane of the equator); $\mathrm{X}_{0}, \mathrm{Y}_{0}, \mathrm{X}_{0}$, and $\mathrm{y}_{0}$ are the preliminary values of the coordinates, and $\gamma^{\prime}$ and $\delta^{\prime}$ are the measured values.

Let us find the partial derivatives contained in Expression $(21.1)$,

$$
\begin{gathered}
-\frac{\partial \gamma}{\partial x_{k}}=\frac{\sin \gamma^{\prime}}{s_{0}}=\frac{\sin \gamma^{\prime}}{\rho_{0} \cos \delta^{\prime}}=-a, \\
\frac{\partial \gamma}{\partial y_{k}}=-\frac{\cos \gamma^{\prime}}{s_{0}}=-\frac{\cos \gamma^{\prime}}{\rho_{0} \cos \delta^{\prime}}=-b, \\
\frac{\partial \gamma}{\partial X_{i}}=-\frac{\sin \gamma^{\prime}}{s_{0}}=-\frac{\sin \gamma^{\prime}}{\rho_{0} \cos \delta^{\prime}}=+a, \\
\frac{\partial \gamma}{\partial Y_{i}}=\frac{\cos \gamma^{\prime}}{s_{0}}=\frac{\cos \gamma^{\prime}}{\rho_{0} \cos \delta^{\prime}}=+b .
\end{gathered}
$$

Taking into account Formulas (21.2), Expression (21.1) acquires the form

$$
\begin{aligned}
v_{\vartheta_{i k}}= & a \xi_{k}+b \eta_{k}-a \xi^{\xi}-b \eta_{i}+l_{\gamma_{i k}} \\
& \text { Weight } p_{\gamma_{i k}}
\end{aligned}
$$

The coefficients of the correction equations are the functions of spherical coordinates $\gamma$ and $\delta$. In some cases, it is feasible to represent them in the form of functions of direction cosines of direction $1 \mathrm{k}$.

Taking into account the known relations (16.1)

$$
\begin{gathered}
l=\cos \delta \cos \gamma, \\
m=\cos \delta \sin \gamma, \\
n=\sin \delta
\end{gathered}
$$


it is easy to obtain

$$
\begin{gathered}
\sin \gamma=\frac{m}{\sqrt{l^{2}+m^{2}}}, \\
\sin \delta=n \\
\cos \gamma=\frac{l}{\sqrt{l^{2}+m^{2}}}, \\
\cos \delta=\sqrt{1-n^{2}} .
\end{gathered}
$$

We substitute (21.4) and Equation (21.3) with consideration of (21.2), and then we obtain the correction equation in the form

$$
v_{v}=\frac{1}{s \sqrt{l^{2}+m^{2}}}-\left(m \xi_{k}-l \eta_{k}-m \xi_{l}+l \eta_{i}\right)+l_{\gamma}
$$

Angle $\delta$ as a function of rectangular coordinates is described by Equation (15.2). Reducing it to linear form, we obtain

$$
\begin{aligned}
v_{\delta_{i k}}= & \frac{\partial \delta}{\partial x_{k}} \xi_{k}+\frac{\partial \delta}{\partial y_{k}} \eta_{k}+\frac{\partial \delta}{\partial z_{k}} \zeta_{k}+\frac{\partial \delta}{\partial X_{l}} \xi_{l}+ \\
& +\frac{\partial \delta}{\partial Y_{l}} \eta_{i}+\frac{\partial \delta}{\partial Z_{i}} \zeta_{l}+l_{\delta_{i k}},
\end{aligned}
$$

where $\zeta=d z$,

$$
l_{\delta}=\operatorname{arctg}\left(\frac{z_{k}-Z_{i}}{\sqrt{\left(x_{k}-\bar{X}_{i}\right)^{2}+\left(y_{k}-Y_{i}\right)^{2}}}\right)-\delta^{\prime}
$$

We find the partial derivatives

$$
\begin{aligned}
& \frac{\partial \delta}{\partial x_{k}}=\frac{-\cos \gamma^{\prime} \sin \delta^{\prime}}{\rho_{0}}=+c ; \\
& \frac{\partial \delta}{\partial X_{i}}=\frac{\cos \gamma^{\prime} \sin \delta^{\prime}}{\rho_{0}}=-c ; \\
& \frac{\partial \delta}{\partial y_{k}}=\frac{-\sin \gamma^{\prime} \sin \delta^{\prime}}{\rho_{0}}=+d ; \\
& \frac{\partial \delta}{\partial Y_{i}}=\frac{\sin \gamma^{\prime} \sin \delta^{\prime}}{\rho_{0}}=-d ; \\
& \frac{\partial \delta}{\partial z_{k}}=\frac{\cos \delta^{\prime}}{\rho_{0}}=+e ; \\
& \frac{\partial \delta}{\partial Z_{i}}=-\frac{\cos \delta^{\prime}}{\rho_{0}}=-c ;
\end{aligned}
$$


With consideration of (21.7), Expression (21.6) is written

$$
v_{\boldsymbol{\delta}_{i k}}=-c \xi_{l}-d \eta_{i}-e \xi_{l}+c \xi_{k}+d \eta_{k}+e \xi_{k}+l_{\delta_{i k}} ; \text { Weight } p_{\delta_{l k}} \text {. }
$$

If the partial derivatives of (21.7) are expressed by the direction cosines of a given straight line in space, using Formulas (21.4) for this, Equation (21.8) acquires the form

$$
\begin{aligned}
v_{\delta_{i k}}= & \frac{n}{\rho \sqrt{l^{2}+m^{2}}}\left(-l \xi_{l}-m \eta_{i}+l \xi_{k}+m \eta_{k}\right)- \\
& -\frac{\sqrt{1-n^{2}}}{\rho}\left(\zeta_{l}-\zeta_{k}\right)+l_{\delta_{i k}}
\end{aligned}
$$

The weights of the measured values for their correction equations are calculated from the relations of the squares of the mean square errors of the measured values. Taking the fact into account that $\mathrm{m}_{\delta}=\mathrm{m}_{\gamma} \cos \delta$ holds as a result of astrometrical processing, the weights of the correcting equations of angular measurements may be written in the form

$$
\begin{array}{r}
p_{s}=\frac{c}{m_{g}^{2}} ; \\
p_{1}=\frac{c}{m_{g}^{2} \sec ^{2} \delta} .
\end{array}
$$

If the observations are made by cameras of the same type, they may be considered balanced, and assuming $p_{\delta}^{2}=1$, consequently, $C=\mathrm{m}_{\delta}^{2}$, we will have $\mathrm{p}_{\gamma}=\cos ^{2} \delta$.

The correction equation for $\gamma$ is not compiled if the time of observation of a passive AES is not recorded. 
If the errors of recording time $\left(m_{s}\right)$ are known, the weights of the correction equations will be calculated as

$$
\begin{gathered}
p_{v}=\frac{c}{\sec ^{2} \delta m_{\delta}^{2}+\left(1+k^{2}\right) m_{S}^{2}} \text { and } \\
p_{\delta}=\frac{c}{m_{\delta}^{2}+\left(k^{\prime}\right)^{2} m_{S}^{2}},
\end{gathered}
$$

where $k$ and $k^{\prime}$ are the components of the angular topocentric speed of motion of the AES along axes $\alpha$ and $\delta$.

Angles $\gamma$ and $\delta$ should be regarded as correlated due to their derivation from astometric processing of the same photograph and their reference to a single moment. However, the results of adjustment hardly change if this correlation is disregarded.

Cases are possible when the direction of the chord between two points, obtained, for example, from ordinary triangulation, is used as the measured element of space triangulation.

In these cases, for angles $\Lambda$ and $\Phi$, which determine the direction of the chord, we may compile correction equations exactly like those for angles $\gamma$ and $\delta$

$$
\begin{gathered}
v_{\Lambda_{l j}}=-a \xi_{i}-b \eta_{i}+a \xi_{j}+b \eta_{j}+l_{\Lambda_{l j}}, \\
v_{\Phi_{i j}}=-C \xi_{i}-d \eta_{i}-e \zeta_{i}+C \xi_{j}+d \eta_{j}+e \zeta_{j}+l_{\Phi_{i j}}
\end{gathered}
$$

in which the coefficlents and free terms are calculated from Formulas (21.1) and (21.2) replacing $\gamma_{1 k}, \delta_{1 k}$ and $\rho_{i k}$ and the Index $k$, respectively, by $\Lambda_{1 j}, \Phi_{i j}, D_{1 j}$ and $j$.

The direction of the chord may also be the initial direction. In this case, the corresponding number of unknowns may be excluded from adjustment. However, to retain uniformity in adjustment. 
It is feasible to compile correction equations for initial directions as well, giving to them such a large welght that they will almost be equal to zero as a result of the correction adjustment.

The Correction Equation for Distance

Reducing Equation (15.3) for measured distance to Iinear form, we have

$$
\begin{aligned}
v_{\xi_{i k}}= & =\frac{\partial \rho}{\partial x_{k}} \xi_{k}+\frac{\partial \rho}{\partial y_{k}} \eta_{k}+\frac{\partial \rho}{\partial z_{k}} \zeta_{k}+\frac{\partial \rho}{\partial X_{l}} \xi_{l}+ \\
& +\frac{\partial \rho}{\partial Y_{i}} \eta_{i}+\frac{\partial_{l}}{\partial Z_{i}} \zeta_{l}+l_{i_{k}} ;, p_{i k},
\end{aligned}
$$

where

$$
l_{f_{i h}}=-\sqrt{\left(x_{k}-X_{i}\right)_{0}^{2}+\left(y_{k}-Y_{i}\right)_{0}^{2}+\left(z_{k}-Z_{i}\right)_{0}^{2}}-\rho_{i h}^{i} .
$$

The partial derivatives of function $\rho$ have a very simple form

$$
\begin{aligned}
& \frac{\partial \dot{\rho}}{\partial x_{k}}=\frac{x_{k}-X_{i}}{\rho_{i k}}=l_{i k}^{\prime} ; \\
& \frac{\partial g}{\partial X_{i}^{\prime}}=-\frac{x_{k}-X_{i}}{\rho_{i k}}=-l_{i k}^{\prime} ; . \\
& \frac{\partial \rho}{\partial y_{k}}=\frac{y_{k}-Y_{i}}{\rho_{i k}}=m_{i k}^{\prime} ; \\
& \frac{\partial \rho}{\partial Y_{i}^{\prime}}=-\frac{y_{k}-Y_{i}}{\gamma_{i k}}=-m_{i k}^{\prime} ; \\
& \frac{\partial \rho}{\partial \bar{z}_{k}}=\frac{z_{k}-Z_{i}}{\rho_{i k}}=n_{i k}^{\prime} ; \\
& \frac{\partial \rho}{\partial Z_{i}}=-\frac{z_{k}-Z_{i}}{\rho_{i k}}=-n_{i k}^{\prime} .
\end{aligned}
$$

Having substituted (21.14) into Expression (21.13), we obtain

$$
\begin{aligned}
& v_{p_{i k}}=l_{i k}^{\prime} \xi_{k}+m_{i k}^{\prime} \eta_{k}+n_{i k}^{\prime} \xi_{k}- \\
& -l_{i k}^{\prime} \xi_{i}-m_{i k}^{\prime} \eta_{i}-n_{i k}^{\prime} \zeta_{i}+l_{i k}, \\
& p_{p}=\frac{c \rho^{2}}{n t_{\mathrm{p}}^{2}} \text { or at } \dot{c}=m_{\delta}^{2} \text { we have } p_{p}=\frac{m_{\dot{\delta}}^{2} \cdot \dot{\rho}^{2}}{m m_{p}^{2}} \text {. }
\end{aligned}
$$


If the relative error of distance measurement is

$$
\frac{m_{p}}{\rho}=M_{\rho}, \text { then } \quad p_{\rho}=\frac{m_{\delta}^{2}}{M_{\rho}^{2}}
$$

To establish the scale of space triangulation, it may include the bases of the "space bases" between the observation points, obtained from a complex of highly accurate linear and angular measurements, made on the Earth's surface. The correction equations for these bases are included in adjustment of space triangulation by the parametric method with a welght corresponding to their accuracy. In the case when the base is taken as the initial value, they are formulated in the same manner as those with the initial direction of the chord.

The correction equation for the measured base corresponds completely to Expression (21.15). However, the unknown values in it, instead of corrections for the position of the AES, will be corrections for the coordinates of the observation point, and the coefficients with unknowns will be the direction cosines of the chord, connecting the ends of the base.

The Polish geodesist Y. A. Zelinskiy [22] proposed using the distances between AES positions, located on a single orbital revolution, as measured values to increase the rigidity of space trlangulation and to establish its scale. These AES positions were observed simultaneously from the space triangulation points by the photographlc method. The value of the distances (the celestial chords between the AES positions) should be determined by the orbital parameters obtained independently of these observations, on the condition of their small differential variation. 
The correlation equation for measured bases of celestial chords obtained will have the form (21.15). However, the unknowns will be corrections for the coordinates of two AES positions, and coefficients $\tau, \mathrm{m}$, and $\mathrm{n}$ will be the direction cosines of the celestial chord.

The correction equation for the measured distance as the base between points in the adjustment of space triangulation by the parametric method may also be used in a single aspect. We know that the heights of points above the reference ellipsoid are known with a considerably higher accuracy than the geodetic coordinates of points on any surface of this ellipsold. Consequently, the preliminary values of the coordinates of the calculated points of space triangulation will contain one component which is more accurate than the two others, and, it is desirable that the corrections of it from the adjustment be less. During adjustment in geocentric coordinates, it is simpler to do this by using the following procedure.

The length of the geocentric radius vector of the point being determined is calculated from its preliminary coordinates.

The center of the Earth (coordinates $X=0, Y=0, Z=0$ ) is taken as the additional initial point, and the length of the radius vector - as the "measured base" between the center of the Earth and the point being determined. When establishing the measurement weight, the accuracy of determining the altitude of the point above sea level and the accuracy of the altitude of the geoid above the ellipsoid at this point are taken into account.

Correction Equation (21.15) in this case has the form

$$
v_{R_{i}}=L_{i}^{0 \xi_{i}}+M_{i}^{0} \eta_{i}+N_{i}^{0} \xi_{i}+l_{R_{i}},
$$


where

$$
l_{R_{i}}=\sqrt{X_{0}^{2}+Y_{0}^{2}+Z_{0}^{2}}-\left(N^{0}+H+h\right) \sqrt{1-\left(2 e^{2}-e^{4}\right) \sin ^{2} B^{0}}
$$

or

$$
v_{R_{i}}^{\prime}=a_{i} \xi_{i}+b_{l} \eta_{i}+c_{i} \xi_{l}+l_{R_{i}}^{\prime}
$$

where

$$
\begin{aligned}
a_{i} & =b_{i}=\frac{1-T_{i}}{R_{l}}+\frac{R_{i} T_{i}^{3}}{a^{2}}, \\
c_{l} & =\frac{1-T_{i}}{R_{i}}+\frac{R_{i} T_{l}^{3}}{a^{2}\left(1-e^{2}\right)}, \\
T_{l} & =\frac{a \sqrt{1-e^{2}}}{\sqrt{\left(1-e^{2}\right)\left(X_{i}^{2}+Y_{i}^{2}\right)-1-Z_{i}^{2}}}, \\
l_{R_{i}}^{\prime} & =R_{i}^{0}\left(1-T_{i}^{0}\right)-(H+h) .
\end{aligned}
$$

Correction Equation for Measured Difference in Distances

To compile the correction equation for the difference in distances, it is necessäry to reduce Expression (15.14) to linear form

$$
\Delta \rho=\rho_{l k}-\rho_{l l},
$$

where $k$ and $z$ are the AES positions, and 1 is the observation point. As a result, we will have

$$
\begin{gathered}
v_{\Delta ?}=\frac{\partial \Delta \rho}{\partial X_{l}} \xi_{i}+\frac{\partial \Delta \rho}{\partial Y_{i}} \eta_{i}+\frac{\partial \Delta \rho}{\partial Z_{i}} \xi_{l}+\frac{\partial \Delta \rho}{\partial x_{k}} \xi_{k}+\frac{\partial \Delta \rho}{\partial y_{k}} \eta_{k}+\frac{\partial \Lambda \rho}{\partial z_{k}} \xi_{k}+ \\
+\frac{\partial \Delta \rho}{\partial x_{l}} \xi_{l}+\frac{\partial \Delta \rho}{\partial y_{l}} \eta_{l}+\frac{\partial \Delta \rho}{\partial z_{l}} \xi_{l}+l_{\Delta ; i},
\end{gathered}
$$

where

$$
\begin{aligned}
& l_{\Delta \varphi}=\left(\sqrt{\left(x_{k}-X_{i}\right)^{2}+\left(y_{k}-Y_{i}\right)^{2}+\left(z_{k}-Z_{i}\right)^{2}}-\right. \\
& -\sqrt{\left.\left(x_{l}-X_{i}\right)^{2}+\left(y_{l}-Y_{i}\right)^{2}+\left(z_{l}-Z_{i}\right)^{2}\right)_{0}-\Delta \rho^{\prime}} .
\end{aligned}
$$


The values of the partial derivatives will be

$$
\begin{gathered}
\frac{\partial \Delta \rho}{\partial X_{i}}=-l_{i k}+l_{i l}=f, \\
\frac{\partial \Delta \rho}{\partial Y_{i}}=-m_{i k}+m_{i l}=g, \\
\frac{\partial \Delta \rho}{\partial Z_{i}}=-n_{i k}+n_{i l}=h, \\
\frac{\partial \Delta \rho}{\partial x_{k}}=-l_{i k}, \quad \frac{\partial \Delta \rho}{\partial x_{l}}=l_{i l}, \\
\frac{\partial \Delta \rho}{\partial y_{k}}=-m_{i k}, \quad \frac{\partial \Delta \rho}{\partial y_{i}}=m_{i l}, \\
\frac{\partial \Delta \rho}{\partial z_{k}}=-n_{i k}, \quad \frac{\partial \Delta \rho}{\partial z_{l}}=n_{i l} .
\end{gathered}
$$

Substituting (21.18) into (21.17), we obtain the final form of the correction equation for the difference in distances

$$
\begin{gathered}
v_{\Delta \rho_{i}}=-f \xi_{i}-g \eta_{i}-h \zeta_{i}+l_{i k} \xi_{k}+m_{i k} \eta_{k}-n_{i k} \xi_{k}-l_{i l} \xi_{l}- \\
-m_{i l} \dot{\eta}_{l}-n_{i l} \xi_{l}+l_{\Delta_{i_{i}}}
\end{gathered}
$$

with the weight $p_{\Delta_{?}}=\frac{C^{2}}{m_{\Delta_{p}^{2}}^{2}}$ or at $C=m_{\delta}^{2} p_{\Delta_{?}}=\frac{m_{0}^{2}}{m_{\Delta ?}^{2}}$.

Consideration of the Effect of Systematic Errors

When it is assumed that there are systematic errors of recording time at one or several points of space triangulation (including initial points), their effect may appear and be eliminated in the adjustment process. This problem may arise in observation of passive AES, where the synchronous moment must be known with high accuracy.

The error $\sigma t_{t_{i}}$ of the time recording affects the accuracy of calculating the topocentric equatorial coordinates of an AES, and 1 t may be calculated from formulas

$$
\Delta \gamma=k \sigma_{i i} ; \quad \Delta \delta=k \sigma^{\prime} \sigma_{i}
$$


where $k$ and $k^{\prime}$, as in (21.11), are the rates of change of the equatorial topocentric coordinates.

If the errors of recording time $\sigma_{t_{i}}$ are included as additional unknowns in adjustment of space triangulation, the correction equations in adjustment by the parametric method, taking into account errors of recorded time, will have the form

$$
\begin{gathered}
v_{v_{i k}}=-a \xi_{i}-b \eta_{i}+a \xi_{k}+b \eta_{k}+k \sigma_{t_{i}}+l_{v i k}, \\
v_{\delta_{l k}}=-c \xi_{l}-d \eta_{i}-e \zeta_{i}+c \xi_{k}+d \eta_{k}+e \zeta_{k}+k^{\prime} \sigma_{t_{i}}+l_{\delta_{i k}}
\end{gathered}
$$

The unknown $\sigma_{t_{1}}$ will be contained in all error equations, compiled for all AES positions observed from point i.

Inclusion of additional unknowns $\sigma_{t}$ increases the number of normal equations by the number of these unknowns.

The unknown error $\sigma_{\rho_{1}}$ or $\sigma_{\Delta \rho_{1}}$ may also be found in the presence of systematic constant errors in measurements of $\rho$ or $\Delta \rho$. The Order of Adjustment

The main process preceding the calculations in the case of adjustment by the parametric method is establishment of the preliminary values of the parameters - the coordinates of all AES positions and observation points.

The preliminary values of coordinates are calculated sequentially, beginning from initial points, from measured values of $\gamma$ and $\delta$ (and if necessary, of both $\rho$ and $\Delta \rho$ ) from the formulas 
of Sections 17 and 18. In some cases, when the AES orbit is predicted with sufficient accuracy, the preliminary coordinates of the AES may also be calculated on the basis of this prediction.

In case of rough preliminary values of coordinates, adjustment of triangulation may be accomplished by approximations so that the equalized values of the coordinates of first approximation are used for new calculations of the coefficients of the correction equations and their free terms.

The number of correction equations is naturally equal to the number of measurements. Solution of these equations under the condition $[\mathrm{pvv}]=\min$ leads to a system of normal equations for correction of coordinates, whose order is equal to $3(s+P)$, where $S$ is the number of AES positions observed and $P$ is the number of observation points to be determined.

Since the AES positions are not related to each other, but are related only to the observation points,* $s$ is usually considerably greater than $P$.

Having numbered the unknowns for correction of the coordinates of AES positions first in order, and those for correction of the coordinates for the points to be determined as the last, we obtain a system of normal equations, whose form is shown schematically in Figure 38.

It is easy to see that the system decomposes into groups of partially independent equations, and therefore, it is ideal for solution by the Pranis-Pranevich method [42]. Solution of such a system of even very high order does not present technical difficulties when using modern computer technology.

* Only measured differences in distances or "measured" lengths of celestial chords may connect adjacent AES positions. 


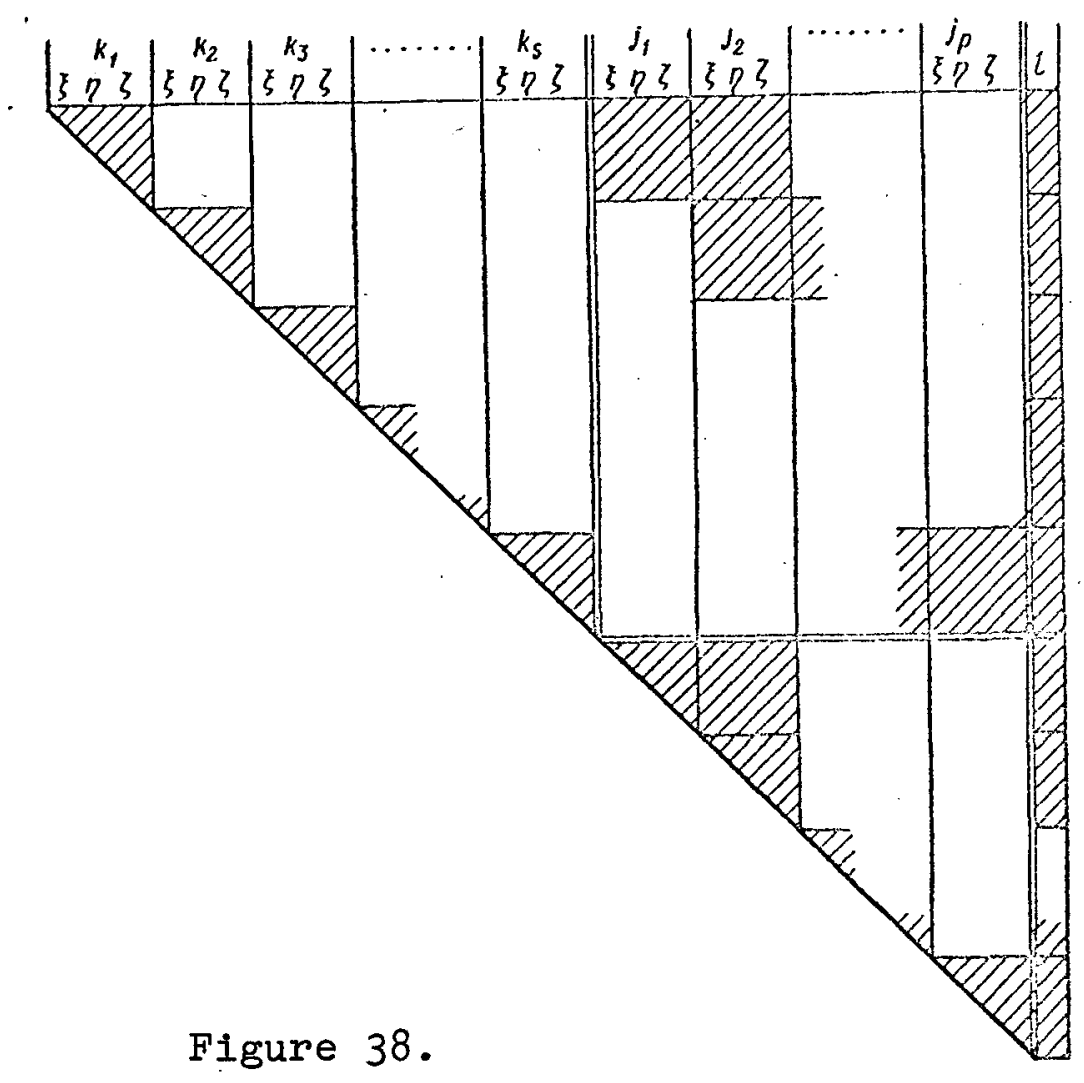

This method of equalization is valid for space triangulation of any construction.

22. Adjustment of Space Triangulation by the Conditional Method with Additional Unknowns

Implementation of the conditional method in its canonical form in the adjustment of angles $\gamma$ and $\delta$ entails considerable difficulties. Introduction of additional unknowns simplifies the compilation of conditional equations. The number of normal equations in this case increases and becomes equal to $N=r+3 P$, where $r$ is the number of independent conditions occurring in a given network, and $P$ is the number of ground points to be determined. 
Since the coordinates of the AES position are intermediate data from the point of view of construction and adjustment of space triangulation, it is feasible to select coordinates of ground points only as additional unknowns. This selection of additional unknowns leads to the fact that three types of conditional equations will be mainly used in mathematical processing: the planar condition and the base conditions for measured distance and for the difference in distances.*

The Conditional Equation of the Synchronization Plane

After reduction to a linear form with respect to corrections for measured values and corrections for the coordinates of points, the conditional equation of the synchronization plane (16.2')

$$
F_{k}=A_{k} \Delta X_{i j}+B_{k} \Delta Y_{i j}+C_{k} \Delta Z_{i j}=0
$$

is written

$$
\begin{aligned}
\alpha_{1} v_{\gamma_{i k}}+\alpha_{2} v_{\delta_{i k}} & +\alpha_{3} v_{\vartheta_{j k}}+\alpha_{k} v_{\delta_{j k}}+A_{k}\left(\xi_{j}-\xi_{l}\right)+B_{k}\left(\eta_{j}-\eta_{i}\right)+ \\
& +C_{k}\left(\zeta_{j}-\zeta_{l}\right)+W_{k}=0
\end{aligned}
$$

where

$$
\begin{gathered}
W_{k}=A_{k}^{\prime} \Delta X^{0}+B_{k}^{\prime} \Delta Y^{0}+C_{k}^{\prime} \Delta Z^{0}, \\
a_{1}=\frac{\partial F}{\partial \gamma_{i k}}=\operatorname{tg} \delta_{j k}\left(\Delta X_{i j} \cos \gamma_{i k}+\Delta Y_{i j} \sin \gamma_{i k}\right)-\Delta Z_{i j} \cos \left(\gamma_{j k}-\gamma_{i k}\right), \\
a_{2}=\frac{\partial F}{\partial \delta_{i k}}=\sec ^{2} \delta_{i k}\left(\Delta X_{i j} \sin \gamma_{j k}+\Delta Y_{i j} \cos \gamma_{j k}\right), \\
\alpha_{3}=\frac{\partial F}{\partial \gamma_{j k}}=-\operatorname{tg} \delta_{l k}\left(\Delta X_{i j} \cos \gamma_{j k}+\Delta Y_{i j} \sin \gamma_{j k}\right)+\Delta Z_{i j} \cos \left(\gamma_{j k}-\gamma_{i k}\right), \\
\alpha_{4}=\frac{\partial F}{\partial \delta_{j k}}=\sec ^{2} \delta_{j k}\left(\Delta X_{i j} \sin \gamma_{i k}-\Delta Y_{i j} \cos \gamma_{i k}\right) \\
A_{k}=\frac{\partial F}{\partial \Delta X_{i j}}=\operatorname{tg} \delta_{j k} \sin \gamma_{i k}-\operatorname{tg} \delta_{i k} \sin \gamma_{j k}, \\
B_{k}=\frac{\partial F}{\partial \Delta Y_{i j}}=\operatorname{tg} \delta_{i k} \cos \gamma_{j k}-\operatorname{tg} \delta_{j k} \cos \gamma_{i k}, \\
C_{k}=\frac{\partial F}{\partial \Delta Z_{i l}}=\sin \left(\gamma_{j k}-\gamma_{i k}\right) .
\end{gathered}
$$

*A special type of condition occurs in the presence of measured directions or bases of chords - conditions of chord elements. The uniqueness of these conditions is that they are related to each other only by the unknowns of triangulation. 
If the measurement contains systematic errors in the determination of the time $\sigma$, conditional Equation (22.1) will assume the form

$$
\begin{array}{r}
a_{1} v_{v_{l k}}+\alpha_{2} v_{\delta_{i k}}+\alpha_{3} v_{v_{j k}}+\alpha_{4} v_{\delta_{j k}}-A \xi_{l}-B \eta_{l}-C \zeta_{l}+A \xi_{j}+ \\
+B \eta_{j}+C \zeta_{l}+\left(\alpha_{1} k_{l}+\alpha_{2} k_{i}^{\prime}\right) \sigma_{l}+\left(\alpha_{3} k_{j}+\alpha_{4} k_{j}^{\prime}\right) \sigma_{i}+W_{k}=0 .
\end{array}
$$

Such equations are complled for all synchronization planes passing through points for which corrections $\sigma_{1}$ and $\sigma_{j}$ are being sought.

Conditional Equations for "Measured" Chord Elements

If the base of a chord connecting two ground points to be determined in a space triangulation network is measured, the base condition will occur in this network.

When using additional unknowns - corrections of the coordinates for the chord ends - the conditional base equation assumes the form of the correction equation for measured distance.

$$
v_{D}-L_{i j}^{0}\left(\xi_{j}-\xi_{l}\right)-M_{i j}^{0}\left(\eta_{j}-\eta_{i}\right)-N_{i j}^{o}\left(\zeta_{j}-\zeta_{i}\right)+W_{D}=0,
$$

where

$$
W_{D}=\sqrt{\left(\Delta X_{i j}^{0}\right)^{2}+\left(\Delta Y_{i j}^{0}\right)^{2}+\left(\Delta Z_{i j}^{0}\right)^{2}}-D_{i j}^{i}
$$

In the case when the base of the chord is measured with a high degree of accuracy (comparable to the accuracy of the initial sides), correction of $v_{D}$ in Equation (22.4) is assumed equal to zero. A similar situation arises in the presence of a "fixed" direction of the chord in triangulation, determined by two angles $\Phi$ and $\Lambda$. In this case, two conditions occur in which the coordinates of the chord ends coincide with angles $\Lambda$ and $\Phi$. When 
additional unknowns are introduced, these conditional equations assume the form of correction equations for measured angles characterizing the direction of chord $\Lambda$ and chord $\Phi$ (21.12), and differ from them only in the fact that corrections $v_{\Lambda}$ and $v_{\Phi}$ vanish.

Thus, the conditional equations are written

$$
\begin{gathered}
-a \xi_{l}-b \eta_{l}+a \xi_{l}+b \eta_{j}+W_{2_{i j}}=0, \\
-c \xi_{l}-d \eta_{l}-e \xi_{l}+c \xi_{j}+d \eta_{j}+e \xi_{j}+W_{\Phi_{i j}}=0,
\end{gathered}
$$

where the expressions for the coefficients may be found in Formulas (21.7).

The Conditional Base Equation for Measured Distance of the AES

In space triangulation constructed from data of simultaneous photographic observations, the scale of which is defined by the system of initial points or bases, the coordinates of all points to be calculated may be obtained. Therefore, the measured distance from an observation point to the position of an AES, the directions toward which have already been determined, is redundant.

The conditional equation with additional unknowns - the coordinates of observation points from which the AES position was observed - may be obtained in a manner similar to the conditional base equation (20.9). From triangle ikj (see Figure 15) we will have

$$
\dot{F}=\frac{\rho_{l k} \sin \beta_{k}}{D_{l j} \sin \beta_{i j}}-1=0
$$

After reducing conditional Equations (22.5) to linear form, 182 we obtain 


$$
\begin{gathered}
\alpha_{1} v_{v k}+\alpha_{2} v_{\delta_{i k}}+\alpha_{3} v_{\gamma_{j k}}+\alpha_{4} v_{j} \delta_{j k}+\alpha_{5} v_{p_{i k}}+a\left(\xi_{j}-\xi_{l}\right)+ \\
+b\left(\eta_{j}-\eta_{i}\right)+c\left(\xi_{j}-\xi_{i}\right)+W=0
\end{gathered}
$$

where

$$
\begin{aligned}
& \alpha_{1}=\frac{\partial F \partial \beta_{k}}{\partial \beta_{k} \partial \gamma_{i k}}=\frac{\operatorname{ctg} \beta_{k}}{\sin \beta_{k}} \cos \delta_{i k} \cos \delta_{j k} \sin \left(\gamma_{j k}-\gamma_{l k}\right), \\
& \alpha_{2}=\frac{\partial F}{\partial \beta_{k}} \cdot \frac{\partial \beta_{k}}{\partial \delta_{i k}}=\frac{\operatorname{ctg} \beta_{k}}{\sin \beta_{k}}\left[\sin \delta_{i k} \cos \delta_{j k} \cos \left(\gamma_{j k}-\gamma_{i k}\right)-\cos \delta_{l k} \sin \delta_{j k}\right] ; \\
& \alpha_{3}=\frac{\partial F}{\partial \beta_{k}} \cdot \frac{\partial \beta_{k}}{\partial \gamma_{j k}}+\frac{\partial F}{\partial \beta_{j}} \frac{\partial \beta_{i}}{\partial \gamma_{j k}}=-\frac{\operatorname{ctg} \beta_{k}}{\sin \beta_{k}} \cos \delta_{i k} \cos \delta_{j k} \sin \left(\gamma_{j k}-\gamma_{i k}\right)+ \\
& +\frac{\operatorname{ctg} \beta_{j}}{D \sin \beta_{j}}\left(\Delta X_{j i} M_{j i}-\Delta Y_{i j} L_{i j}\right) \\
& \alpha_{k}=\frac{\partial F \partial \beta_{k}}{\partial \beta_{k} \partial \delta_{j k}}+\frac{\partial F \partial \beta_{j}}{\partial \beta_{j} \partial \delta_{j k}}=\left[\sin \delta_{j k} \cos \delta_{i k} \cos \left(\gamma_{j k}-\gamma_{i k}\right)-\right. \\
& \left.-\cos \delta_{j k} \sin \delta_{i k}\right]+\frac{\operatorname{ctg} \beta_{j} \operatorname{tg} \delta_{j k}}{D \sin \beta_{j}} \times \\
& \times\left(\Delta X_{i j} L_{i j}+\Delta Y_{i j} M_{i j}-\frac{\cos \delta_{j k}}{\operatorname{tg} \delta_{j k}} \Delta Z_{i j}\right) \\
& \alpha_{5}=\frac{\partial F}{\partial \rho_{i k}}=\frac{1}{\rho_{i k}} \text {, } \\
& a=\frac{\partial F \partial D}{\partial D \partial \Delta X_{l i}}+\frac{\partial F \partial \beta_{j}}{\partial \beta_{j} \partial \Delta X_{i j}}=L_{i j}+ \\
& +\frac{\operatorname{ctg} \beta_{j}}{D \sin \beta_{j}}\left(l_{j k}+L_{j i} \cos \beta_{j}\right) \\
& b=\frac{\partial F \partial D}{\partial D \partial \Delta Y_{i j}}+\frac{\partial F \partial \beta_{i}}{\partial \beta_{j} \partial \Delta Y_{i j}}=M_{i j}+ \\
& +\frac{\operatorname{ctg} \beta_{j}}{D \sin \beta_{j}}\left(m_{j k}+M_{i i} \cos \beta_{j}\right) \\
& c=\frac{\partial F \partial D}{\partial D \partial \Delta Z_{i l}}+\frac{\partial F \partial \beta_{I}}{\partial \beta_{j} \partial \Delta Z_{i j}}=N_{i j}+. \\
& +\frac{\operatorname{ctg} \beta_{l}}{D \sin \beta_{j}}\left(n_{j k}+N_{i l} \cos \beta_{j}\right) \text {. } \\
& W=\frac{\rho_{i h}^{\prime}}{D_{j l}^{0}} \times \frac{\sqrt{1-\left(l_{i h}^{\prime} l_{j k}^{\prime}+m_{i k}^{\prime} m_{j h}^{o}+n_{i h}^{\prime} n_{j h}^{\prime}\right)^{2}}}{\sqrt{1-\left(l_{j h}^{\prime} L_{j i}^{0}+m_{j k}^{\prime} M_{j i}^{o}+n_{j h}^{\prime} N_{j i}^{0}\right)^{2}}}-1 \text {. }
\end{aligned}
$$


The conditional equation of measured distance may be obtained $/ 83$ in another form, by eliminating the coordinates of the AES position from the basic equations of (18.1), after which three equations remain

$$
\begin{aligned}
\Delta X_{i j} & =\rho_{i k} l_{i k}-\rho_{j k} l_{j k}, \\
\Delta Y_{i j} & =\rho_{l k} m_{i k}-\rho_{j k} m_{j k}, \\
\Delta Z_{i j} & =\rho_{l k} n_{i k}-\rho_{j k} n_{j k} .
\end{aligned}
$$

After both distances have been eliminated from these three equations, we obtain the conditional equation of the synchronization plane.

Calculation of one of the distances, for example $\rho_{j k}$ (if distance $\rho_{1 k}$ is measured), from the first equation of (22.10)

$$
\rho_{j k}=\frac{\rho_{i k} l_{l k}-\Lambda X_{i j}}{l_{i k}}
$$

and substitution of it into the second equation leads to the conditional equation of measured distance in the form

$$
F^{\prime}=\left(\Delta X_{i j}-\rho_{t k} l_{i k}\right) m_{j k}-\left(\Delta Y_{i j}-\rho_{i k} m_{i k}\right) l_{j k}=0
$$

Reducing it to linear form, we will have a conditional equation in the form of (22.7), where 


$$
\begin{gathered}
\alpha_{1}=\frac{\partial F^{\prime} \partial l_{i k}}{\partial l_{i k} \partial \gamma_{i k}}+\frac{\partial F^{\prime} \partial \dot{m}_{i k}}{\partial m_{i k} \partial \gamma_{i k}}=\rho_{i k} \cos \delta_{i k} \cos \delta_{j k} \cos \left(\gamma_{j k}-\gamma_{i k}\right) \\
\alpha_{2}=\frac{\partial F^{\prime} \partial l_{i k}}{\partial l_{i k} \partial \delta_{i k}}+\frac{\partial F^{\prime} \partial m_{i k}}{\partial m_{i k} \partial \delta_{i k}}=\rho_{i k} \sin \delta_{i k} \cos \delta_{j k} \cos \left(\gamma_{j k}-\gamma_{i k}\right) \\
\alpha_{3}=\frac{\partial F^{\prime} \partial l_{j k}}{\partial l_{j k} \partial \gamma_{j k}}+\frac{\partial F^{\prime} \partial m_{j k}}{\partial m_{j k} \partial \gamma_{j k}}=-\rho_{i k} \cos \delta_{i k} \cos \delta_{j k} \cos \left(\gamma_{j k}-\gamma_{i k}\right)+\frac{1}{1} \\
+\Delta X_{i j} l_{i k}+\Delta Y_{i j} m_{j k} \\
\alpha_{4}=\frac{\partial F^{\prime} \partial l_{j k}}{\partial l_{j k} \partial \delta_{j k}}+\frac{\partial F^{\prime} \partial m_{j k}}{\partial m_{j k} \partial \delta_{j k}}=\operatorname{tg} \delta_{j k}\left[\Delta Y_{i j} l_{j k}-\Delta X_{i j} m_{j k}+\right. \\
+\rho_{i k} \cos \delta_{i k} \cos \delta_{j k} \sin \left(\gamma_{j k}-\gamma_{i k}\right) \\
\alpha_{5}=\frac{\partial F^{\prime}}{\partial p_{i k}}=\cos \delta_{i k} \cos \delta_{j k} \sin \left(\gamma_{i k}-\gamma_{i k}\right) \\
a=\frac{\partial F^{\prime}}{\partial \Delta \lambda_{i j}}=m_{j k} \\
b=\frac{\partial F^{\prime}}{\partial \Delta \nu_{i j}^{\prime}}=-l_{j k} \\
c=\frac{\partial F^{\prime}}{\partial \Delta Z_{i j}}=0 \\
W=\left(\Lambda X_{i j}^{0}-\rho_{i k}^{\prime} l_{i k}^{\prime}\right) m_{j k}^{\prime}- \\
-\left(\Delta Y_{i j}^{0}-\rho_{i k}^{\prime} m_{i k}^{\prime}\right) l_{j k}^{\prime} .
\end{gathered}
$$

In the case when, besides the directions to the AES position, 184 both distances to this position $\rho_{1 k}$ and $\rho_{\mathrm{jk}}$ are calculated from two observation points, a conditional equation of the form (22.6) or the form (22.12) may be complled for each of the distances. Moreover, one of these equations may be replaced by the condition which expresses the cosine formula for a plane triangle. This condition is obtained as a result of adding the squares of the three basic equations of (18.1)

$$
\varphi=D_{i j}^{\dot{i}}-\rho_{i k}^{2}-\rho_{j h}^{2}+2 \rho_{i k} \rho_{j k} \cos \beta_{k} \text {. }
$$

Reduction of (22.15) to Iinear form yields the conditional equation

$$
\begin{aligned}
& \alpha_{1} v_{\gamma_{b k}}+\alpha_{2} v_{\delta_{i k}}+\alpha_{3} v_{\gamma_{j k}}+\alpha_{4} v_{\delta_{j k}}+ \\
& +\alpha_{5} v_{p_{i k}}+\alpha_{b} v_{p_{j k}}+a\left(\xi_{j}-\xi_{l}\right)+ \\
& +b\left(\eta_{j}-\eta_{l}\right)+c\left(\zeta_{j}-\zeta_{i}\right)+W=0
\end{aligned}
$$




\section{where}

$$
\begin{aligned}
& \alpha_{1}=\frac{\partial \varphi \partial \beta_{k}}{\partial \beta_{k} \partial \gamma_{i k}}=\rho_{i k} \rho_{j k} \cos \delta_{i k} \cos \delta_{j k} \sin \left(\gamma_{l k}-\gamma_{j k}\right), \\
& \alpha_{2}=\frac{\partial \varphi \partial \rho_{k}}{\partial \rho_{j} \partial \delta_{i k}}=-\rho_{i k} \rho_{j k}\left[\sin \delta_{i k} \cos \delta_{j k} \cos \left(\gamma_{i k}-\gamma_{j k}\right)-\cos \delta_{l k} \sin \delta_{j k}\right] \text {, } \\
& \alpha_{3}=\frac{\partial \varphi \partial \beta_{k}}{\partial \beta_{k} \partial \gamma_{j k}}=\rho_{i k} \rho_{j k} \cos \delta_{i k} \cos \delta_{j k} \sin \left(\gamma_{j k}-\gamma_{i k}\right), \\
& \alpha_{4}=\frac{\partial \varphi \partial \beta_{k}}{\partial \beta_{k} \partial \delta_{j k}}=-\rho_{i k} \rho_{j k}\left[\sin \delta_{j k} \cos \delta_{l k} \cos \left(\gamma_{j k}-\gamma_{l k}\right)-\cos \delta_{j k} \sin \delta_{l k}\right] \text {, } \\
& \alpha_{5}=\frac{\partial \varphi}{\partial \rho_{i k}}=\rho_{j k} \cos \beta_{k}--\rho_{l k} \text {. } \\
& \alpha_{i}=\frac{\partial \varphi}{\partial \rho_{i k}}=\rho_{i k} \cos \beta_{k}-\rho_{j k} \text {, } \\
& a=\frac{\partial \varphi \partial D}{\partial D \partial \Delta \bar{X}_{l i}}=\Delta X_{i j} \\
& b=\frac{\partial \varphi \partial D}{\partial D \partial \Delta Y_{i j}}=\Delta Y_{i j} \\
& c=\frac{\partial \varphi \partial D}{\partial D \partial \Delta Z_{l j}}=\Delta Z_{i j},
\end{aligned}
$$

$$
W=\frac{1}{2}\left[D_{i j}^{0^{2}}-\rho_{i k}^{\prime 2}-\rho_{j k}^{\prime 2}+2 \rho_{i h}^{\prime} \rho_{j k}^{\prime}\left(l_{i k}^{\prime} l_{j k}^{\prime}+m_{i k}^{\prime} m_{j k}^{\prime}+n_{i k}^{\prime} n_{j k}^{\prime}\right)\right]
$$

Finally, for this same condition, the equations (22.10) may be used directly as conditions

$$
\begin{aligned}
& \varphi_{1}=\Delta X_{l j}-\left(\rho_{i k} l_{i k}-\rho_{j k} l_{j k}\right)=0 \\
& \varphi_{2}=\Delta \dot{Y_{l j}}-\left(\rho_{l k} m_{l k}-\rho_{j k} m_{j k}\right)=0 \\
& \varphi_{3}=\Delta \dot{Z}_{l j}-\dot{-}\left(\rho_{l k} n_{l k}-\rho_{i k} n_{j k}\right)=0 .
\end{aligned}
$$

After reducing them to Iinear form, we obtain

$$
\begin{aligned}
& \alpha_{1} v_{v_{l k}}+\alpha_{2} v_{\delta_{l k}}+\alpha_{3} v_{v_{j k}}+\alpha_{4} v_{j}+ \\
& +\alpha_{5} v_{P_{i k}}+\alpha_{0} v_{g_{j k}}+a_{1}\left(\xi_{j}-\xi_{j}\right)+ \\
& +b_{1}\left(\eta_{j}-\eta_{i}\right)+c_{1}\left(\xi_{j}-\xi_{i}\right)+W_{\alpha}=0 \text {, } \\
& \beta_{1} v_{\gamma_{l k}}-\beta_{2} v_{i_{i k}}+\beta_{3} v_{v_{j k}}+\beta_{z} v_{z_{j k}}+ \\
& +\beta_{5} v_{\rho_{i k}}+\beta_{6} v_{\rho_{j k}}+a_{2}\left(\xi_{i}-\xi_{i}\right)+ \\
& +b_{2}\left(\eta_{j}-\eta_{i}\right)+c_{2}\left(\zeta_{j}-\zeta_{i}\right)+W_{\beta}=0 \text {, } \\
& \varepsilon_{1} v_{\gamma_{i k}}+\varepsilon_{2} v_{z_{i k}}+\varepsilon_{3} v_{\gamma_{j k}}+\varepsilon_{1} v_{j k}+ \\
& +\varepsilon_{5} v_{\rho_{i k}}+\varepsilon_{6} v_{P_{j k}}+a_{3}\left(\xi_{j}-\xi_{i}\right)+ \\
& +b_{3}\left(\eta_{j}-\eta_{l}\right)+c_{3}\left(\zeta_{j}-\xi_{l}\right)+W_{\varepsilon}=0 \text {. }
\end{aligned}
$$


where

$$
\begin{gathered}
\alpha_{1}=\rho_{i k} \cos \delta_{i k} \sin \gamma_{i k}, \\
\alpha_{2}=\rho_{i k} \sin \delta_{i k} \cos \gamma_{i k}, \\
\alpha_{3}=-\rho_{j k} \cos \delta_{j k} \sin \gamma_{j k}, \\
\alpha_{4}=-\rho_{j k} \sin \delta_{j k} \cos \gamma_{j k}, \\
\alpha_{5}=-\cos \delta_{i k} \cos \gamma_{i k}, \\
\alpha_{6}=\cos \delta_{j k} \cos \gamma_{j k}, \\
a_{1}=1, b_{1}=0, c_{1}=0, \\
\beta_{1}=-\rho_{i k} \cos \delta_{i k} \cos \gamma_{i k}, \\
\beta_{2}=\rho_{i k} \sin \delta_{i k} \sin \gamma_{i k}, \\
\beta_{3}=\rho_{j k} \cos \delta_{i k} \cos \gamma_{j k}, \\
\beta_{4}=-\rho_{j k} \sin \delta_{j k} \sin \gamma_{j k}, \\
\beta_{5}=-\cos \delta_{i k} \sin \gamma_{i k}, \\
\beta_{6}=\cos \delta_{j k} \sin \gamma_{j k}, \\
a_{2}=0, b_{2}=1, c_{2}=0, \\
\varepsilon_{1}=0, \\
\varepsilon_{2}=-=-\rho_{i k} \cos \delta_{i k}, \\
\varepsilon_{3}=0, \\
\varepsilon_{4}=\rho_{j k} \cos \delta_{j k}, \\
\varepsilon_{5}=-\sin \delta_{i k}, \\
\varepsilon_{6}=\sin \delta_{j k},
\end{gathered}
$$

$$
\begin{aligned}
& W_{i}=\Delta X_{i j}^{o}-\rho_{i k}^{\prime} \cos \delta_{i h}^{\prime} \cos \gamma_{i k}^{\prime} \mu \\
& +\rho_{j:}^{\prime} \cos \delta_{j i}^{i} \cos \gamma_{j k}^{\prime} \text {, } \\
& W_{3}^{\prime}=A Y_{i j}^{0}-\rho_{i k}^{\prime} \cos \delta_{i k}^{\prime} \sin \gamma_{i k}^{\prime}-i \\
& +\rho_{j k}^{\prime} \cos \delta_{j k}^{\prime} \sin \gamma_{j k}^{\prime} \text {, } \\
& W_{k}==\Delta Z_{i j}^{0}-\rho_{i k}^{\prime} \sin \delta_{i k}^{\prime}+\rho_{j k}^{\prime} \sin \delta_{j k}^{\prime} \text {. }
\end{aligned}
$$

Conditional Equation of the Difference in Distances

Let us consider a space triangulation figure (see Flgure 16), in which photographic observation of two AES positions is accomplished from two ground points, and, moreover, the difference in 
distances to these same AES positions is calculated from one of the ground points. Two conditions of the synchronization plane and one base condition for the measured difference in distances occur in this figure, which has the form

$$
F=\Delta \rho_{i}+D \Delta X_{i j}+E \Delta Z_{i j}=0
$$

(Another equation with functions of other coordinates may also be obtained), where

$$
\begin{aligned}
& D=\frac{\cos \delta_{i k_{1}} \sin \gamma_{i k_{1}}}{\sin \left(i_{j k_{1}}-\gamma_{i k_{1}}\right)}-\frac{\cos \delta_{i k_{2}} \sin \gamma_{j k_{2}}}{\sin \left(\gamma_{j k_{2}}-\gamma_{i k_{2}}\right)}, \\
& E=\frac{\cos \delta_{i k_{1}} \cos \gamma_{i k_{1}}}{\sin \left(\gamma_{j k_{1}}-\gamma_{i k_{1}}\right)}+\frac{\cos \delta_{i k_{2}} \cos \gamma_{j k_{2}}}{\sin \left(\gamma_{j k_{2}}-\gamma_{i k_{2}}\right)} .
\end{aligned}
$$

After reduction to Iinear form, we obtain the conditional correction equation

$$
\begin{aligned}
& -\alpha_{1} v_{\gamma_{i k_{1}}}+\alpha_{2} v_{i_{i k_{2}}}+\alpha_{3} v_{\gamma_{i k_{2}}}+\alpha_{4} v_{\delta_{j k_{1}}}+\alpha_{5} v_{\gamma_{i k_{2}}}+\alpha_{6} v_{\delta_{i k_{8}}}+ \\
& +\alpha_{i} v_{\gamma_{j k_{z}}}+\alpha_{8} v_{\delta_{\boldsymbol{j}_{z}}}+\alpha_{8} v_{\Delta_{i} i}+a\left(\xi_{j}-\xi_{i}\right)+b\left(\xi_{j}-\xi_{i}\right)+W=0 \text {, }
\end{aligned}
$$

In which

$$
\begin{aligned}
& \alpha_{1}=\frac{\partial F}{\partial \gamma_{i k_{1}}}=\frac{\cos \delta_{i k_{i}} \cos \left(\gamma_{i k_{1}}-\gamma_{i k_{1}}\right)}{\sin ^{2}\left(\gamma_{j k_{1}}-\gamma_{i k_{1}}\right)}\left(\Delta X_{i j} \sin \gamma_{j k_{2}}+\Delta Z_{i j} \cos \gamma_{j k_{1}}\right), \\
& a_{2}=-\frac{\partial F}{\partial \delta_{i k_{1}}}=-\frac{\sin \delta_{i k_{1}}}{\sin \left(\gamma_{j k_{1}}-\gamma_{i k_{1}}\right)}\left(\Delta X_{i j} \sin \gamma_{i h_{1}}+\Delta Z_{i j} \cos \gamma_{j k_{1}}\right), \\
& \alpha_{3}=\frac{\partial F}{\partial \gamma_{j k_{1}}}=-\frac{\cos \delta_{i h_{1}}}{\sin ^{2}\left(\gamma_{j k_{1}}-\gamma_{i k_{3}}\right)}\left(\Delta X_{l j} \sin \gamma_{i h_{1}}+\Delta Z_{i j} \cos \gamma_{i k_{1}}\right), \\
& \alpha_{4}=\frac{\partial F}{\partial \delta_{j k_{1}}}=0 \text {, } \\
& a_{6}=\frac{\partial F}{\partial Y_{i k_{2}}}=\frac{\cos \delta_{i k_{1}} \cos \left(\gamma_{j k_{2}}-\gamma_{i k_{2}}\right)}{\sin ^{2}\left(\gamma_{j k_{2}}-\gamma_{i k_{2}}\right)}\left(-\Delta X_{i j} \sin \gamma_{j h_{1}}+\Delta Z_{l j} \cos \gamma_{j k_{2}}\right) \text {, } \\
& \alpha_{6}=\frac{\partial F}{\partial \delta_{i k_{z}}}=-\frac{\sin \delta_{i k_{z}}}{\sin \left(\gamma_{j k_{2}}-\gamma_{i k_{z}}\right)}\left(-\Delta X_{l j} \sin \gamma_{j k_{z}}+\Delta Z_{l j} \cos \gamma_{j k_{8}}\right), \\
& \alpha_{7}=\frac{\partial F}{\partial \gamma_{j k_{2}}}=-\frac{\cos \delta_{i h_{2}}}{\sin ^{2}\left(\gamma_{i k_{2}}-\gamma_{i k_{2}}\right)}\left(-\Delta X_{i j} \sin \gamma_{i h_{2}}+\Delta Z_{i j} \cos \gamma_{i h_{2}}\right) \text {, } \\
& \alpha_{8}=\frac{\partial F}{\partial \delta_{j k_{2}}}=0 \text {, } \\
& \alpha_{\theta}=\frac{\partial F}{\partial \Delta \rho_{i}}=1 \text {, } \\
& a=\frac{\partial F}{\partial \Delta X_{i j}}=D \text {, } \\
& b=\frac{\partial F}{\partial \Delta Z_{i j}}=E \text {. }
\end{aligned}
$$

$$
W=\Delta \varphi_{i}^{i}+D^{\prime} \Delta X_{i j}^{0}+E^{\prime} \Delta Z_{i j}^{0} \text {. }
$$


In complex space triangulation figures, we may encounter the most varled combination of measured directions and differences in distances. However, the conditional equations occurring in this case may be reduced in the final analysis to conditional equations of synchronization planes and to conditional equations of the differences in distances, similar to those which we have presented.

The Order of Adjustment

Preliminary values of the coordinates of the observation points to be determined should first be established in order to compile conditional equations. This may be accomplished by different methods, for example, by sequential transfer of the coordinates from initial points through elementary space triangulation figures, omitting calculation of AES coordinates.

An important process preceding adjusting calculations is the selection of conditional equations and enumeration of their number.

The number of equations of the synchronization planes may be calculated by the formula

$$
p=\sum_{i}^{s} s_{i}\left[m_{i}+\left(m_{i}-3\right)\right]
$$

where $s$ is the number of observed AES; and $m$ is the number of directions converging at a given AES position. The number of base conditional equations for chord bases, the measured distances to the AES, or the difference in distances is equal to the number of these values.

The welghts of the measured values are established by the rules outlined in section 21 . 
Joint solution of the conditional equations under the condition

$$
[p v v]-2 \sum k_{r}\left\{[\alpha v]+a_{r} \xi_{t}+b_{r} \eta_{i}+c_{r} \zeta_{l}\right\}=\min
$$

leads to a system of normal correlation equations and coordinate corrections of the observation points, which has order $r+3 P$, where $r$ is the number of correlates equal to the number of conditional equations, and $P$ is the number of observation points to be calculated.

By combining into separate groups the conditional equations containing corrections for the same measurements, partial independence of the normal equations corresponding to them is achieved.

The described method is rigorous for adjustment of space triangulation networks of any construction.

\section{Adjustment of Space Triangulation, Formed by} Planes by the Parametric Method

If space triangulation is constructed by simultaneous photographic observations of each AES position from two observation points only, the conditional equations of the synchronization planes are independent of each other.

Having expressed the conditional equation of plane (22.1) in explicit form with respect to corrections for additional unknowns, we obtain

$$
-A_{k} \xi_{i}-B_{k} \eta_{i}-C_{k} \xi_{i}+A_{k} \xi_{j}+B_{k} \eta_{\jmath}+C_{k} \zeta_{j}+l_{k}=\varepsilon_{k}
$$

where $A_{k}, B_{k}$, and $C_{k}$ are the coefficlents of the plane equations which are calculated by Formulas (22.2). The free term is 


$$
l_{k}=A_{k}^{\prime}\left(X_{j}^{0}-X_{i}^{0}\right)+B_{k}^{\prime}\left(Y_{i}^{0}-Y_{i}^{0}\right)+C_{h}^{\prime}\left(Z_{i}^{0}-Z_{i}^{0}\right),
$$

where $\mathrm{X}^{0}, \mathrm{Y}^{0}$, and $\mathrm{Z}^{0}$ are the preliminary values of the coordinates of points 1 and $j$. The right side of the equation is

$$
\varepsilon=-\left(\alpha_{1} v_{i k}+\alpha_{2} v_{i k}+\alpha_{3} v_{\gamma_{i k}}+\alpha_{4} v_{\delta_{j k}}\right)
$$

where $\alpha_{1}$ are the partial derivatives whose values are calculated by Formulas (22.2).

If the measurements have systematic time errors, the equation assumes the form

$$
\begin{gathered}
\mathbf{\varepsilon}_{k}=-A_{k} \xi_{l}-B_{k} \eta_{l}-C_{k} \zeta_{l}+A_{k} \xi_{j}+B_{k} \eta_{j}+C_{k} \zeta_{l}+\left(\alpha_{1} k_{l}+\alpha_{2} k_{i}^{\prime}\right) \sigma_{t_{i}}+ \\
+\left(\alpha_{3} k_{j}+\alpha_{1} k_{j}^{\prime}\right) \sigma_{l_{j}}+l_{k} .
\end{gathered}
$$

The unique feature of Equation (23.I) is that the value which it describes is actually not measured. It is a single geometric image (plane), obtained on the basis of several measurements. Nevertheless, Expression (23.1) may be considered as a correction equation both formally and in essence, since the value $\varepsilon_{k}$ expresses the error of calculating the given plane.

The welght of the equation of the synchronization plane is calculated by the expression

$$
p_{k}=\frac{1}{\frac{\alpha_{1}^{8}}{p_{\gamma_{l k}}}+\frac{\alpha_{2}^{2}}{p_{\delta_{l k}}^{2}}+\frac{\alpha_{3}^{2}}{p_{\Upsilon_{j k}}}+\frac{\alpha_{l}^{2}}{p_{\delta / k}}} .
$$

Joint solution of the system of correction equations (23.1) under the condition $\left[\mathrm{p}_{k} \varepsilon_{k} \varepsilon_{k}\right]=\mathrm{min}$, which is equivalent to condition $\left[p_{\gamma} v_{\gamma} v_{\gamma}\right]+\left[p_{\delta} v_{\delta} v_{\delta}\right]=\min$, leads to a system of normal equations whose order will be equal only to the number of the unknown coordinates of the observation points to be calculated, i.e., $3 \mathrm{P}$. 
If there are separate AES positions in the space triangulation network observed from more than two observation points, and (or) there are linear measurements, the combined method of adjustment by the parametric method may be used, when the synchronization planes and direct measurements are considered jointly as measured values. When adjusting for AES positions, observed from three or more observation points, by this method, as well as for linear measurements, the correction equations are compiled in the same way as in the parametric method. The equations for correction of planes (23.1) are compiled for AES positions observed from two observation points only. Two partial systems of normal equations are compiled for the two groups of correction equations obtained. The first system includes the unknown corrections of the coordinates of observation points and AES positions. This system is solved until elimination of the corrections of the coordinates of AES positions. Afterwards, all the coefficients of the transformed system are added to the coefficients in the case of the corresponding corrections of the second partial system. The order of the connecting system of normal equations obtained, whose solution gives the corrections of the observation points to be determined, will be equal to $3 P$.

The combined method will also be rigorous for adjustment of triangulation of any construction.

24. Adjustment of Space Triangulation Constructed from Chords

If each AES position in space triangulation is observed from two points only, all measured values may be divided into groups, each of which is related only to a single chord, connecting the observation points. Adjustment may be carried out in two stages, with such a construction of space triangulation. In the first stage, the most probable values of the unknowns which characterize 
the position of the chord itself are calculated as a result of adjustment of the values measured directly. The second stage consists of adjustment of a three-dimensional triangulation network constructed from chords. This method of adjustment (if simultaneous photographic observations only are carried out in the network) has been named the "method of closing directions" in the literature.

The orientation angles of chord $\Lambda$ and $\Phi$ and its base $D$ are used as the "measured values" in adjustment of triangulation of chords (in the second stage). However, all these values will be dependent, because their values are calculated as a result of adjustment of the same measurements in the first stage. Therefore, as a result of the first stage, along with the most probable values of corrections of values $\Lambda^{0}, \Phi^{0}$, and $D^{0}$, we must calculate 190 the elements of the correlation matrix

$$
Q_{\Lambda \Phi D}=\left[\begin{array}{lll}
q_{\Lambda \Lambda} & q_{\Lambda \Phi} & q_{\Lambda D} \\
q_{\Phi \Lambda} & q_{\Phi \Phi} & q_{\Phi D} \\
q_{D \Lambda} & q_{D \Phi} & q_{D D}
\end{array}\right]
$$

which is required for the second stage of adjustment.

The parametric and conditional methods are applicable in both the first and second stages of adjustment of space triangulation constructed from chords.

Let us consider specific uses of these methods.

\section{Adjustment in the First Stage by the Parametric Method}

If space triangulation is constructed only from simultaneous photographic observations, each measured value $\gamma$ and $\delta$ is contained only in a single equation of the synchronization plane and, consequently, these equations will be independent. 
In this case, each planar equation of (16.9)

$$
F_{k}=\operatorname{tg} \delta_{i k} \sin \left(\Lambda_{i j}-\gamma_{j k}\right)+\operatorname{tg} \delta_{j k} \sin \left(\gamma_{i k}-\Lambda_{i j}\right)+\operatorname{tg} \Phi_{i j} \sin \left(\gamma_{j k}-\gamma_{i k}\right)=0
$$

may be represented in the form of correction equations. By reducing 1 t to linear form, we obtain

$$
\varepsilon_{k}=a_{k} \Delta \Lambda_{l_{j}}+b_{k} \Delta \Phi_{t_{j}}+l_{k} \text {, we1ght } p_{k},
$$

where

$$
\left.\begin{array}{c}
a_{k}=\frac{\partial F_{k}}{\partial \Lambda_{i j}}=\operatorname{tg} \delta_{i k} \cos \left(\gamma_{j k}-\Lambda_{i j}\right)+\operatorname{tg} \delta_{j k} \cos \left(\gamma_{i k}-\Lambda_{i j}\right) \\
b_{k}=\frac{\partial F_{k}}{\partial \Phi_{i j}}=-\frac{\sin \left(\gamma_{j k}-\gamma_{i k}\right)}{\cos ^{2} \Phi_{i j}}
\end{array}\right\},
$$

The values $\Lambda_{i f}^{0}$ and $\Phi_{i f}^{0}$ are calculated from the preliminary coordinates of points $i$ and $j$ according to Expressions (16.13). The weight of each Equation (24.2) is found from Formula (23.5), in which

$$
\begin{gathered}
\alpha_{1}=\frac{\partial F_{k}}{\partial \gamma_{i k}}=\operatorname{tg} \delta_{i k} \cos \left(\gamma_{l k}-\Lambda_{i j}\right)-\operatorname{tg} \Phi_{l j} \cos \left(\gamma_{j k}-\gamma_{i k}\right), \\
\alpha_{2}=\frac{\partial F_{k}}{\partial \delta_{i k}}=\frac{\sin \left(\Lambda_{i j}-\gamma_{i k}\right)}{\cos ^{2} \delta_{i k}}, \\
\alpha_{3}=\frac{\partial F_{k}}{\partial \gamma_{j k}}=-\operatorname{tg} \delta_{i k} \cos \left(\Lambda_{i j}-\gamma_{l k}\right)+\operatorname{tg} \Phi_{l i} \cos \left(\gamma_{j k}-\gamma_{i k}\right), \\
\alpha_{4}=\frac{\partial F_{k}}{\partial \delta_{j k}}=\frac{\sin \left(\gamma_{i k}-\Lambda_{i j}\right)}{\cos ^{2} \delta_{i k}} .
\end{gathered}
$$

Solution of the system of Equations (24.2) under the condi- 191 tion $\left[p_{k} \varepsilon_{k} \varepsilon_{k}\right]=$ min leads for each chord to a system of two normal equations, as a result of which the correction values of $\Delta_{\Lambda}$ and $\Delta_{\Phi}$ are obtained, as well as the elements of the correlation matrix 


$$
Q_{\Lambda \Phi}=\left[\begin{array}{ll}
q_{\Lambda \Lambda} & q_{\Lambda} \\
q_{\Phi \Lambda} & q_{\downarrow \alpha}
\end{array}\right]
$$

If space triangulation contains both the angular measured values $(\gamma, \delta)$ and Iinear values $(\rho, \Delta \rho)$, the conditional equations of the synchronization planes become dependent, because the measured values $\gamma$ and $\delta$ will also be contained in the conditional equations which occur from being combined with the distances and differences in distances. In this case, the plane equations may not be considered as correction equations, and corrections of directly measured values may not be expressed in explicit form only by the unknowns $\Lambda, \Phi$, and $D$, which characterize the direction and length of the chord.

For this situation, the coordinates of AES positions and the coordinates of one of the observation points at the end of the chord may be selected as unknowns in the first stage in adjustment by the parametric method. The second point should be considered as the reference point, because we are interested in the given case only in the mutual position of the chord ends.

The equations for correcting the measured values $v_{\gamma}, v_{\delta}$, $\mathrm{v}_{\rho}$, and $\mathrm{v}_{\Delta \rho}$ have exactly the same form as adjustment of the entire triangulation by the parametric method (21.3), (21.8), (21.15), and (21.19). However, these equations do not contain corrections of the coordinates of point (i), and the corrections of the coordinates of the other point ( $j$ ) actually become the corrections of the differences in the coordinates $\left(\xi_{j}=d \Delta X_{i j}\right.$, $\left.\eta_{j}=d \Delta \ddot{Y}_{i j}, \zeta_{j}=d \Delta Z_{i j}\right)$. 
As a result of solving the system of normal equations for each chord separately, the value of the differences in coordinates $\Delta \mathrm{X}, \Delta \mathrm{Y}$, and $\Delta \mathrm{Z}$ and their correlation matrix are calculated

$$
Q_{\Delta X, \Delta Y, \Delta Z}=\left[\begin{array}{lll}
q_{\Delta X \Delta X} & q_{\Delta X \Delta Y^{*}} & q_{\Delta X \Delta Z} \\
q_{\Delta Y \Delta X} & q_{\Delta Y \Delta X} \Delta Y_{\Delta Y \Delta Z} \\
q_{\Delta Z \Delta X} & q_{\Delta Z \Delta Y^{*}} & q_{\Delta Z \Delta Z}
\end{array}\right]
$$

In conclusion, the values of $\Lambda, \Phi$, and $D$, which correspond to the adjusted values of $\Delta X, \Delta Y$, and $\Delta Z$ from Formulas (16.13), are calculated for the second stage of adjustment.

To obtain the correlation matrix (24.1), and taking the fact into account that $\Lambda, \Phi$, and $D$ are complex functions related to the measurements by $\Delta X, \Delta Y$, and $\Delta Z$, by differentiation of (16.13), we find

$$
\left[\begin{array}{l}
d \Lambda \\
d \Phi \\
d D
\end{array}\right]=\left[\begin{array}{lll}
f_{11} & f_{12} & f_{13} \\
f_{21} & f_{22} & f_{23} \\
f_{31} & f_{32} & f_{33}
\end{array}\right] \cdot\left[\begin{array}{c}
\xi \\
\eta \\
\zeta
\end{array}\right]
$$

where

$$
\begin{gathered}
f_{11}=\frac{\partial \Lambda}{\partial \Delta X}=-\frac{\Delta Y}{\Delta X^{2}+\Delta Y^{2}} ; \\
f_{12}=\frac{\partial \Lambda}{\partial \Delta Y}=\frac{\Delta X}{\Delta X^{2}+\Delta Y^{2}} ; \\
f_{13}=\frac{\partial \Lambda}{\partial \Delta Z}=0 ; \\
f_{21}=\frac{\partial \Phi}{\partial \Delta X}=-\frac{\Delta X+\Delta Z}{\left(\Delta X^{2}+\Delta Y^{2}+\Delta Z^{2}\right) \sqrt{\Delta X^{2}+\Delta Y^{2}}} ; \\
f_{22}=\frac{\partial \Phi^{\prime}}{\partial \Delta Y^{2}}=-\frac{\Delta Y+\Delta Z}{\left(\Delta X^{2}+\Delta Y^{2}+\Delta Z\right) \sqrt{\Delta X^{2}+\Delta Y^{2}}} ; \\
f_{23}=-\frac{\partial \Phi}{\partial \Delta Z}=\frac{\sqrt{\Delta X^{2}+\Delta Y^{2}}}{\Delta X^{2}+\Delta Y^{2}+\Delta Z^{2}} ; \\
f_{31}=\frac{\partial D}{\partial \Delta X}=\frac{\Delta X}{\sqrt{\Delta X^{2}+1-\Delta Y^{2}+\Delta Z^{2}}} ; \\
f_{32}=\frac{\partial D}{\partial \Delta Y}=\frac{\Delta Y}{\sqrt{\Delta X^{2}+\Delta Y^{2}+\Delta Z^{2}}} ; \\
f_{33}=\frac{\partial D}{\partial \Delta Z}=\frac{\Delta Z}{\sqrt{\Delta \bar{X}^{2}+\Delta Y^{2}+\Delta Z^{2}}} ;
\end{gathered}
$$


Now,

$$
\begin{aligned}
Q_{\Lambda \Phi D} & =\left[\begin{array}{lll}
f_{11} & f_{12} & f_{13} \\
f_{21} & f_{22} & f_{23} \\
f_{31} & f_{32} & f_{33}
\end{array}\right] \cdot\left[\begin{array}{lll}
q_{\Delta X \Delta X} & q_{\Delta X \Delta Y} & q_{\Delta X \Delta Z} \\
q_{\Delta Y \Delta X} & q_{\Delta Y \Delta Y} & q_{\Delta Y \Delta Z} \\
q_{\Delta Z \Delta X} & q_{\Delta Z \Delta Y} & q_{\Delta Z \Delta Z}
\end{array}\right] \times \\
& \times\left[\begin{array}{lll}
f_{11} & f_{12} & f_{13} \\
f_{21} & f_{22} & f_{23} \\
f_{31} & f_{32} & f_{33}
\end{array}\right]=\left[\begin{array}{lll}
q_{\Lambda \Lambda} & q_{\Lambda \Phi} & q_{\Lambda D} \\
q_{\Phi \Lambda} & q_{\Phi \Phi} & q_{\Phi D} \\
q_{D \Lambda} & q_{D \Phi} & q_{D D}
\end{array}\right] .
\end{aligned}
$$

Adjustment in the First Stage by the Conditional Method with Additional Unknowns

Depending on the composition of measurements in the space triangulation figure from which the given chord is calculated, plane conditions and base conditions for the measured distances and their differences may occur. It is obvious that corrections must be introduced into the orientation angles $\Delta_{\Lambda}$ and $\Delta_{\varphi}$ and correction in its base $\Delta_{D}$ in this case, as additional unknowns. In the general case, the conditional equation with additional unknowns for any composition of measurements will be

$$
\sum a_{k} v_{l k}+a_{r} \Lambda_{\Lambda}+b_{r} \Delta_{\Phi}+c_{r} \Lambda_{D}+W_{r}=0,
$$

where $v_{k}$ are corrections of the values measured directly, and the coefficients $\alpha_{k}$ are calculated by the formulas of Section 22. The values $a_{r}, b_{r}$, and $c_{r}$ are partial derivatives of the form $a=\partial F / \partial \Lambda, b=\partial F / \partial \Phi$, and $c=\partial F / \partial D$, which may be easily obtained from consideration of expressions for specific geometric conditions.

One correlate in the system of the normal correlation equations and corrections of additional unknowns will correspond to each conditional equation. Thus, a system of normal equations will have an order equal to $r+3$, where $r$ is the number of 
conditions and 3 corresponds to the number of additional unknowns - corrections $\Delta_{\Lambda_{i j}}, \Delta_{\Phi_{i j}}$, and $\Delta_{D_{i j}}$.

As a result of solving the system of equations under the condition

$$
[p v v]-2 \sum_{i j} i_{r}\left([a v]+a_{r} \Delta_{\Lambda}+b_{r} \Delta_{\Phi}+c_{r} \Lambda_{D}\right)=\text { min }
$$

for each chord, the corrections of orientation angles $\left(\Delta_{\Lambda}, \Delta_{\Phi}\right)$ and chord base $\left(\Delta_{D}\right)$ of interest to us, as well as the correlation matrix (24.1), will be obtained in addition to the values of the correlates.

Adjustment of Chords in the Second Stage by the Parametric Method

In adjustment of space triangulation constructed from chords, the values of $\Lambda^{\prime}, \Phi^{\prime}$, and $D^{\prime}$, found from adjustment in the first stage, are used as the measured values in the second stage. The correction equations for this case are presented in Expressions (21.12) and (21.15), where the symbols $\tau_{i k}, m_{i k}$, and $n_{i k}$ should be replaced by $L_{i j}, M_{i j}$, and $N_{i j}$. The free terms of the correction equations are calculated by the formulas

$$
\begin{aligned}
& l_{\Lambda}=\Lambda^{0}-\Lambda^{\prime} \\
& l_{\Phi}=\Phi^{0}-\Phi^{\prime} \\
& l_{D}=D^{0}-D^{\prime},
\end{aligned}
$$

where $\Lambda^{0}, \Phi^{0}$, and $D^{0}$ are the orientation angles of the chord and its length, obtained from the preliminary coordinates of ground points. 
Since the values of $\Lambda, \Phi$, and $D$ for each chord will be dependent, the generalized least squares principle $\mathrm{v}^{\mathrm{T}} \mathrm{Q}^{-1} \mathrm{v}=\mathrm{min}$ must be used for joint solution of the system of correction equations. The use of this condition leads to a system of normal equations of order $3 P$ ( $P$ is the number of observation points to be calculated)

$$
A^{T} Q^{-1} A X=A^{T} Q^{-1} L
$$

where $A$ is the matrix of the coefficients of correction equations, $L$ is the vector of the free terms of the same equations, and $X$ is the vector of corrections in the coordinates of ground points. The elements of the correlation matrix $Q$ are calculated in the first stage of adjustment.

We note in conclusion that the reciprocal correlation matrix $Q^{-1}$ is simpler to calculate if the correction equations, in which the unknowns are corrections $\Delta_{\Lambda}, \Delta_{\Phi}$, and $\Delta_{D}$, are used in the first stage of adjustment. Actually, the system of correction equations, related to the given chord, is written

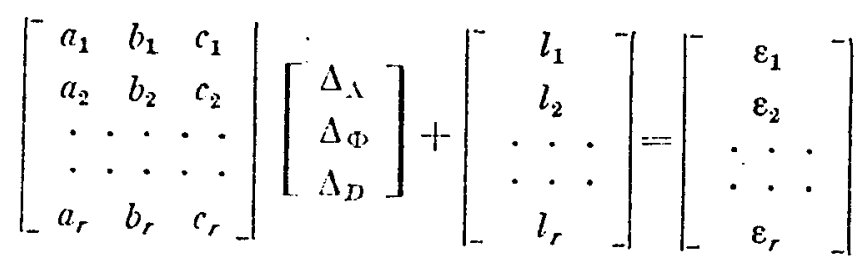

or in generalized form

$$
a \Delta+L=\varepsilon
$$

The normal system, corresponding to (24.15), will be

$$
a^{T} P_{\mathrm{\varepsilon}} a \Delta+a^{T} P_{\varepsilon} L=0
$$


Hence, we find the correction vector $\Delta\left\{\Delta_{\Lambda}, \Delta_{\Phi}, \Delta_{D}\right\}$,

$$
\Delta=-\left(a^{T} P_{8} a\right)^{-1} a^{T} P_{\varepsilon} L=-Q a^{T} P_{\varepsilon} L,
$$

where $Q$ is the correlation matrix (the matrix of weighting factors) of system (24.16).

The reciprocal correlation matrix $Q^{-1}$, which is required for adjustment of chords in the second stage by the parametric method, has the form

$$
Q^{-1}=\left[\left(a^{T} P_{\mathrm{s}} a\right)^{-1}\right]^{-1}=a^{T} P_{\varepsilon} a
$$

Thus, if corrections $\Delta_{\Lambda}, \Delta_{\Phi}$, and $\Delta_{D}$ are calculated directly in the first stage, matrix $Q^{-1}$ coincides with the matrix of normal equations of the first stage.

Adjustment of Chords in the Second Stage by the Conditional Method

In adjustment of space triangulation constructed from chords, introduction of additional unknowns - corrections in the ground point coordinates - is useless, because the planar equation passing through three observation points is satisfied for any values of the coordinate of these observation points. Therefore, the conditional equations of coplanarity of three free vectors; as well as the polar, base and coordinate conditional equations must be used in the second stage. Calculation of the number of conditions of each type may be carried out by the same rules as for two-dimensional networks. We should add that the polar and base conditions in a space network formed by chords are perceived "by the eye" as similar conditions in a two-dimensional network, and the conditions of the coplanarlty of three free vectors are perceived as the conditions of triangles with variable angles. 
The coplanarity condition of three chords, belonging to a single three-dimensional triangle (the condition of the plane of three observation points), according to (20.5), has the form

$$
F=\left[\begin{array}{lll}
L_{1} & L_{2} & L_{3} \\
M_{1} & M_{2} & M_{3} \\
N_{1} & N_{2} & N_{3}
\end{array}\right]=0
$$

where $L_{1}, M_{1}$, and $N_{1}$ are the direction cosines of the chords cal- 195 culated from the orientation angles of chord $\Lambda^{\prime}$ and $\Phi^{\prime}$, adjusted In the first stage. By reducint (24.19) to a linear form with respect to corrections in the orientation angles $v_{\Lambda}$ and $v_{\Phi}$, we obtain

$$
\sum_{1}^{3} \frac{\partial F}{\partial \Lambda_{l}} v_{\Lambda_{l}}+\sum_{1}^{3} \frac{\partial F}{\partial W_{l}}-v_{\Phi_{i}}+W=0
$$

Representing the partial derivatives of (24.19) in the form

$$
\begin{aligned}
& \frac{\partial F}{\partial \Lambda}=\frac{\partial F}{\partial L} \cdot \frac{\partial L}{\partial \Lambda}+\frac{\partial F}{\partial M} \cdot \frac{\partial M}{\partial \Lambda}+\frac{\partial F}{\partial N} \cdot \frac{\partial N}{\partial \Lambda} \\
& \frac{\partial F}{\partial \Phi}=\frac{\partial F}{\partial L} \cdot \frac{\partial L}{\partial \Phi}+\frac{\partial F}{\partial M} \cdot \frac{\partial M}{\partial \Phi}+\frac{\partial F}{\partial N} \cdot \frac{\partial N}{\partial \Phi},
\end{aligned}
$$

we obtain

$$
\begin{gathered}
\frac{\partial F}{\partial \Lambda_{l}}=-M_{i} A_{i}+L_{i} B_{i} \\
\frac{\partial F}{\partial \Phi_{i}}=\frac{C_{l}}{\cos \Phi_{t}},
\end{gathered}
$$

where $A, B$ and $C$ are found from Expressions (16.3) with replacement of symbols $2, m$, and $n$ by $L, M$, and $N$ in them. The free term of Equation (24.20) is calculated by the formula

$$
W=L^{\prime} A^{\prime}+M^{\prime} B^{\prime}+N^{\prime} C^{\prime}
$$


In which all values are calculated from the values of $\Lambda^{\prime}$ and $\Phi^{\prime}$, obtained in the first stage of adjustment.

The conditional base and polar equations formally have the same form (otherwise, only the free term will be calculated). Expanding Expression (20.9) in a serles, we obtain

$$
\sum \frac{\partial F}{\partial \Lambda_{i}} v_{\Lambda_{l}}+\sum \frac{\partial F}{\partial \Phi_{l}} v_{\Phi_{l}}+W=0
$$

The coefficients of Equation (24.24) may be represented by

$$
\begin{aligned}
& \frac{\partial F}{\partial \Lambda_{l}}=\sum_{i}^{t}\left(\frac{\partial F}{\partial \beta_{k}} \cdot \frac{\partial \beta_{k}}{\partial L_{i}} \cdot \frac{\partial L_{i}}{\partial \Lambda_{i}}+\frac{\partial F}{\partial \beta_{k}} \cdot \frac{\partial \beta_{k}}{\partial M_{l}} \cdot \frac{\partial M_{l}}{\partial \Lambda_{l}}+\frac{\partial F}{\partial \beta_{k}} \cdot \frac{\partial \beta_{k}}{\partial N_{l}} \cdot \frac{\partial N_{l}}{\partial \Lambda_{l}}\right) \\
& \frac{\partial F}{\partial \Phi_{l}}=\sum_{i}^{t}\left(\frac{\partial F}{\partial \beta_{k}}: \frac{\partial \beta_{k}}{\partial L_{l}} \cdot \frac{\partial L_{i}}{\partial \Phi_{l}}+\frac{\partial F}{\partial \beta_{k}} \cdot \frac{\partial \beta_{k}}{\partial M_{l}} \cdot \frac{\partial M_{i}}{\partial \Phi_{l}}+\frac{\partial F}{\partial \beta_{k}} \cdot \frac{\partial \beta_{k}}{\partial N_{l}} \cdot \frac{\partial N_{i}}{\partial \Phi_{l}}\right),
\end{aligned}
$$

where $\beta_{k}$ are the angles of the three-dimensional triangles formed by the chords and $t$ is the number of angles in which the given chord is contained.

Having calculated the partial derivatives in (24.25), we find the following expressions for the coefficients of Equation $(24.24)$

$$
\begin{gathered}
\frac{\partial F}{\partial \Lambda_{l}}=\sum_{1}^{t}(-1)^{k} \frac{\operatorname{ctg} \beta_{k}}{\sin \beta_{k}}\left(L_{l} M_{l}-L_{i} M_{l}\right) \\
\frac{\partial F}{\partial \Psi_{i}}=\sum_{\substack{l \\
l}}^{t}(-1)^{k} \frac{\operatorname{ctg} \beta_{k}}{\sin \beta_{k} \cos \Phi_{l}}\left(N_{l}-N_{l} \cos \beta_{k}\right),
\end{gathered}
$$

where 1 and $f$ are the numbers of the chords which form angle $\beta_{k}$.

If the bases of the chords are measured in the network, Equation (24.24) assumes the form

$$
\sum \frac{\partial F}{\partial \Lambda_{i}} v_{\Lambda_{l}}+\sum_{i d} \frac{\partial F}{\partial \Phi_{i}} v_{\Phi_{i}}+\frac{v_{D_{1}}}{D_{1}}+\frac{v_{D_{2}}}{D_{2}}+W=0,
$$


where $v_{D_{1}}$ are the corrections for the measured chord bases.

The coordinate conditions for a network constructed from chords have the form

$$
\begin{gathered}
\varphi_{X}=\sum L_{i} D_{l}-\Delta X \\
\varphi_{Y}=\sum M_{i} D_{i}-\Delta Y, \\
\varphi_{Z}=\sum N_{i} D_{i}-\Delta Z .
\end{gathered}
$$

Henceforth, we will use the conditional equation only for the abscissas, since the other equations may be obtained in a similar manner.

By reducing the function $\varphi_{X}$ to linear form, we obtain the following conditional equation

$$
\sum_{i=0} \frac{\partial \varphi_{X}}{\partial \Lambda_{l}} v_{\Lambda_{i}}+\sum_{i=d} \frac{\partial \varphi_{X}}{\partial \Phi_{l}} v_{\Phi_{i}}+W_{X}=0
$$

In which

$$
\begin{aligned}
& \frac{\partial \varphi_{X}}{\partial \Lambda_{i}}=\frac{\partial L_{l}}{\partial \Lambda_{i}} D_{l}+\frac{\partial D_{l}}{\partial \Lambda_{i}} L_{l} \\
& \frac{\partial \varphi_{X}}{\partial \Phi_{i}}=\frac{\partial L_{l}}{\partial \Phi_{l}} D_{l}+\frac{\partial D_{i}}{\partial \Phi_{l}} L_{l} .
\end{aligned}
$$

Having calculated the partial derivatives which are contained in $(24.30)$, we obtain.

$$
\begin{gathered}
\frac{\partial \varphi_{X}}{\partial \Lambda_{l}}=-M_{l} D_{l}+L_{i} D_{l} \sum_{1}^{t}(-1)^{2 h-1} \frac{\operatorname{ctg} \beta_{k}}{\sin \beta_{k}}\left(L_{i} M_{i}-L_{i} M_{l}\right), \\
\vdots \\
\frac{\partial \varphi_{X}}{\partial \Phi_{l}}=-N_{l} D_{l} \cos \Lambda_{i}+L_{l} D_{i} \sum_{i}^{t}(-1)^{2 h-1} \frac{\operatorname{ctg} \beta_{k}}{\sin \beta_{k} \cos \Phi_{l}}\left(N_{i}-N_{l} \cos \beta_{k}\right),
\end{gathered}
$$

where the symbols $t, 1, j$, and $\beta_{k}$ have the same meanings as in Expressions $(24.26)$. 
If the chord bases in the network are measured, the form of the coordinate conditional equations will be simplified considerably; thus, the equation for the abscissas is written

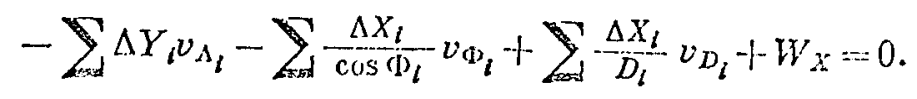

The set of conditional equations which occur in the given space triangulation network is written in matrix form

$$
a V+W=0
$$

where $\alpha$ is the matrix of the coefficients of the conditional equations, $V$ is the correction vector for values $\Lambda$ and $\Phi$, and $W$ is the vector of free terms. Joint solution of Equations (24.33) under the conditions of the generalized least squares principle $\mathrm{V}^{\mathrm{T}} \mathrm{Q}^{-1} \mathrm{~V}=\min$ leads to a system of normal correlate equations

$$
\alpha Q a^{T} K+W=0
$$

In which $K$ is the correlate vector and $Q$ is the correlation matrix, obtained in the first stage of adjustment. Having calculated the correlate vector from System (24.34), it is easy to find the desired correction vector for the orientation angles of chords $\Lambda, \Phi$, and $D$

$$
V=Q \alpha^{T} K
$$

We can avoid the use of the correlation matrix of "measured values" in the second stage of adjustment by introducing a special local coordinate system for each chord in the first stage of adjustment. Introduction of this system, one of whose axes is aligned along the displacement of the end of the chord 
in terms of altitude ( $h$ ), and the second - along the displacement in terms of azimuth (a), was proposed by L. Aardom, A. Hirnius, and $G$. Vels for adjustment of space triangulation constructed by the Smithsonian Astrophysical Observatory [46]. Due to the fact that the axes of the error ellipse in the direction of chord if are oriented along axes $h$ and $a$ in a plane perpendicular to the direction of the chord at point $j$, correlation between corrections for the direction of chord $d h$ and da will be negligible.

Let us consider the relationship between corrections of the orientation angles of chord $\Lambda$ and $\Phi$, and corrections of $d$ h and da.

The corrections of $d \Phi$ and $d \Lambda \cos \Phi$ are also located in a plane perpendicular to the direction of the chord at point $j$, along the axes of a plane rectangular coordinate system, which are the traces of intersection of this plane by a plane passing through the chord parallel to the $z$-axis of a geocentric coordinate system $(z)$ and a plane passing through a chord perpendicular to the first plane (s), respectively.

The axes $\mathrm{h}$ and $\mathrm{a}$ in a plane perpendicular to the direction of the chord are traces of a plane passing through the chord and the origin of geocentric rectangular coordinates and of a plane passing through the chord perpendicular to the first, respectively. Thus, the axis $h$ is approximately perpendicular to the Earth's surface.

The coordinate system $h, a$ is rotated with respect to the system $z$, a by angle a between a plane passing through the chord and the origin of the ground coordinate system, and a plane passing through the chord parallel to the $\mathrm{Z}$-axis. This angle may be found from the expression 


$$
\begin{aligned}
& \cos \alpha= \\
& =\frac{\left(Y_{i} Z_{j}-Y_{j} Z_{i}\right)\left(Y_{j}-Y_{l}\right)+\left(Z_{i} X_{j}-Z_{j} X_{i}\right)\left(X_{i}-X_{j}\right)}{\sqrt{\left[\left(Y_{i} Z_{j}-Y_{j} Z_{i}\right)^{2}+\left(Z_{i} X_{j}-Z_{j} X_{i}\right)^{2}+\left(X_{i} Y_{j}-X_{j} Y_{i}\right)^{2}\right]\left[\left(Y_{j}-Y_{i}\right)^{2}+\left(X_{i}-Y_{j}\right)^{2}\right]}} \cdot \quad(24.36)
\end{aligned}
$$

For adjustment in the first stage, let us represent the correction equation of the synchronization plane (23.1), having divided it by $D_{1 j}$, in the form

$$
\varepsilon_{k}^{\prime}=A_{k} d L_{i j}+B_{k} d M_{i j}+C_{k} d N_{i j}+W_{k}^{\prime}, \text { weight } P_{k}^{-}
$$

Transition from corrections of the direction cosines of the chord to corrections of the orientation angles $\Lambda_{1 j}$ and $\Phi_{1 j}$ is calculated by the expression

$$
\left[\begin{array}{l}
d L \\
d M \\
d \Lambda^{\prime}
\end{array}\right]=\left[\begin{array}{rrr}
-\sin \Lambda & -\sin \Phi & \cos \Lambda \\
\cos \Lambda & -\sin \Phi & \sin \Lambda \\
0 & \cos \Phi &
\end{array}\right] \cdot\left[\begin{array}{c}
d \Lambda \cos \Phi \\
d \Phi
\end{array}\right] \text {. }
$$

Accordingly, transition from corrections of orientation angles to corrections of $\mathrm{dh}_{1 j}$ and $\mathrm{da}_{1 \mathrm{j}}$ is accomplished by the formula

$$
\left[\begin{array}{c}
d \Lambda \cos \Phi \\
d \Phi
\end{array}\right]=\left[\begin{array}{rr}
-\sin \alpha & \cos \alpha \\
\cos \alpha & \sin \alpha
\end{array}\right] \cdot\left[\begin{array}{l}
d h \\
d u
\end{array}\right]
$$

The correction equations in the first stage, on the basis of (24.37) and taking into account (24.38) and (24.39), will be 


$$
\begin{aligned}
\varepsilon_{k}^{\prime}= & \left\{A\left[\frac{\partial L}{\partial \Lambda \cos \Phi} \cdot \frac{\partial \Lambda \cos \Phi}{d h}+\frac{\partial L}{\partial \Phi} \cdot \frac{\partial \Phi}{\partial h}\right]+\right. \\
+ & +B\left[\frac{\partial N}{\partial \Lambda \cos \Phi} \cdot \frac{\partial \Lambda \cos \Phi}{\partial h}+\frac{\partial M}{\partial \Phi} \cdot \frac{\partial \Phi}{\partial h}\right]+ \\
+ & \left.C\left[\frac{\partial N}{\partial \Lambda \cos \Phi} \cdot \frac{\partial \Lambda \cos \Phi}{\partial h}+\frac{\partial N}{\partial \Phi} \cdot \frac{\partial \Phi}{\partial h}\right]\right\} d h+ \\
+ & \left\{A\left[\frac{\partial L}{\partial \Lambda \cos \Phi)} \cdot \frac{\partial \Lambda \cos \Phi}{\partial a}+\frac{\partial L}{\partial \Phi} \cdot \frac{\partial \Phi}{\partial a}\right]+\right. \\
+ & B\left[\frac{\partial M}{\partial \Lambda \cos \Phi)} \cdot \frac{\partial \Lambda \cos \Phi}{\partial a}+\frac{\partial M}{\partial \Phi} \cdot \frac{\partial \Phi}{\partial a}\right]+ \\
+C & {\left.\left[\frac{\partial N}{\partial \Lambda \cos \Phi} \cdot \frac{\partial \Lambda \cos \Phi}{\partial a}+\frac{\partial N}{\partial \Phi} \cdot \frac{\partial \Phi}{\partial a}\right]\right\} d a+W^{\prime}=}
\end{aligned}
$$

$=[A(\sin \Lambda \sin \alpha-\sin \Phi \cos \Lambda \cos \alpha)-B(\cos \Lambda \sin \alpha+\sin \Phi \sin \Lambda \cos \dot{\alpha})+$

$+C \cos \Phi \cos \alpha] d h+[-A(\sin \Lambda \cos \alpha+\sin \Phi \cos \Lambda \sin \alpha)+$

$+B(\cos \Lambda \cos \alpha-\sin \Phi \sin \Lambda \sin \alpha)+C \cos \Phi \sin \alpha] d a+W^{\prime}$,

where

$$
\begin{gathered}
\varepsilon_{k}^{\prime}=-\left(L \frac{\partial A}{\partial \gamma_{i k}}+M \frac{\partial B}{\partial \gamma_{i k}}+N \frac{\partial C}{\partial \gamma_{i k}}\right) v \gamma_{i k}- \\
-\left(L \frac{\partial A}{\partial \delta_{i k}}+M \frac{\partial B}{\partial \delta_{i k}}+N \frac{\partial C}{\partial \delta_{i k}}\right) v \delta_{i k}-\left(L \frac{\partial A}{\partial \gamma_{i k}}+M \frac{\partial B}{\partial \gamma_{j k}}+N \frac{\partial C}{\partial \gamma_{j k}}\right) v \gamma_{i k}- \\
-\left(L \frac{\partial A}{\partial \delta_{j k}}+M \frac{\partial B}{\partial \delta_{j k}}+N \frac{\partial C}{\partial \delta_{j k}}\right) v_{j k}
\end{gathered}
$$

and the values of the partial derivatives are given in (22.2).

As a result of the first stage for the direction of each chord, we obtain the corrections of $d h_{1 j}$ and $d a_{i f}$ and the matrix

$$
Q=\left[\begin{array}{ll}
q_{h} & 0 \\
0 & q_{a}
\end{array}\right]
$$

or the weights $p_{h}$ and $p_{a}$.

The values of $d h$ and da in the second stage are regarded as "Independent measured" values. 
For their adjustment by the parametric method, the following correction equations are complied.

$$
\begin{gathered}
v_{h_{i j}}=\left(\frac{\partial h}{\partial \Phi)} \cdot \frac{\partial \Phi}{\partial \Delta \bar{X}}+\frac{\partial h_{i}}{\partial \Lambda \cos \Phi} \cdot \frac{\partial \Lambda \cos \Phi}{\partial \Delta X}\right)\left(\xi_{j}-\xi_{i}\right) \div \\
+\left(\frac{\partial h}{\partial()} \cdot \frac{\partial \Phi}{\partial \Delta Y}+\frac{\partial h}{\partial \Lambda \cos \Phi} \cdot \frac{\partial \Lambda \cos \Phi}{\partial \Delta Y}\right)\left(\eta_{j}-\eta_{l}\right) \div \\
+\left(\frac{\partial h}{\partial \Phi} \cdot-\frac{\partial \Phi}{\partial \Lambda Z}+\frac{\partial h}{\partial \Lambda \cos \Phi} \cdot \frac{\partial \Lambda \cos \Phi}{\partial \Delta Z}\right)\left(\zeta_{j}-\xi_{i}\right)+d h_{i j}, p_{h_{i j}} \\
v_{a_{i j}}=\left(\frac{\partial a}{\partial \Phi} \cdot \frac{\partial \Phi}{\partial \Delta X}+\frac{\partial a}{\partial \Lambda \cos \Phi} \cdot \frac{\partial \Lambda \cos \Phi}{\partial \Delta X}\right)\left(\xi_{j}-\xi_{i}\right) \div \\
+\left(\frac{\partial a}{\partial \Phi} \cdot \frac{\partial \Phi}{\partial \Delta Y}+\frac{\partial a}{\partial \Lambda \cos \Phi} \cdot \frac{\partial \Lambda \cos \Phi}{\partial \Delta Y}\right)\left(\eta_{j}-\eta_{i}\right)+ \\
+\left(\frac{\partial a}{\partial \Phi} \cdot \frac{\partial \Phi}{\partial \Delta Z}+\frac{\partial a}{\partial \Lambda \cos \Phi} \cdot \frac{\partial \Lambda \cos \Phi}{\partial \Delta Z}\right)\left(\xi_{i}-\xi_{i}\right)+d a_{i j}, p_{a_{i j}} .
\end{gathered}
$$

Taking into account that

$$
\left[\begin{array}{l}
d h \\
d a
\end{array}\right]=\left[\begin{array}{rr}
\cos \alpha & -\sin \alpha \\
\sin \alpha & \cos \alpha
\end{array}\right] \cdot\left[\begin{array}{c}
d \Phi \\
d \Lambda \cos \Phi
\end{array}\right]
$$

and

$$
\begin{gathered}
{\left[\begin{array}{c}
d \Phi \\
d \Lambda \cos \Phi
\end{array}\right]=\left[\begin{array}{cc}
-\frac{\Delta X \Delta Z}{D^{2} \sqrt{\Delta X^{2}+\Delta Y^{2}}}-\frac{\Delta Y \Delta Z}{D^{2} V^{\left.\Delta X^{2}+\Delta\right)^{2}}} & \frac{\sqrt{\Delta X^{2}-\Delta Y^{2}}}{D^{2}} \\
-\frac{\Delta Y}{D \sqrt{\Delta X^{2}+\Delta Y^{2}}} & 0
\end{array}\right] \times} \\
\quad \times\left[\begin{array}{c}
\left(\xi_{j}-\xi_{i}\right) \\
\left(\eta_{j}-\eta_{i}\right) \\
\left(\xi_{j}-\xi_{i}\right)
\end{array}\right]
\end{gathered}
$$

the equations will have the form

$$
\begin{aligned}
& v_{h_{l j}}=\frac{1}{D \sqrt{\Delta X^{2}+\Delta X^{2}}}\left(-\frac{\Delta X \Delta Z}{D} \cos \alpha+\Delta Y \sin \alpha\right)\left(\xi_{j}-\xi_{i}\right)- \\
& -\frac{1}{D \sqrt{\Delta X^{2}+1-\Delta Y^{2}}}\left(\frac{\Delta Y \Delta Z}{D} \cos \alpha+\Delta X \sin \alpha^{2}\right)\left(\eta_{j}-\eta_{i}\right)+ \\
& +\frac{\sqrt{\Delta X^{2}+\Delta Y^{2}}}{D^{2}} \cos \alpha\left(\zeta_{j}-\zeta_{i}\right)+d h_{l j}, \\
& v_{a_{l J}}=\frac{-1}{D \sqrt{\Delta X^{2} \cdot 1 \cdot \Delta \bar{Y}^{2}}}\left(\frac{\Delta X \Delta Z}{D} \sin \alpha+\Delta Y \cos \alpha\right)\left(\xi_{j}-\xi_{l}\right)+ \\
& +\frac{1}{D \sqrt{\Delta X^{2}+\Delta Y^{2}}}\left(-\frac{\Delta Y^{2} \Delta Z}{D} \sin \alpha+\Delta Y \cos \alpha\right)\left(\eta_{j}-\eta_{i}\right) \div \\
& +\frac{\sqrt{\left.\Delta X^{2}+1-\Delta\right)^{2}}}{D^{2}} \sin \alpha\left(\zeta_{j}-\zeta_{i}\right)+d a_{i j}
\end{aligned}
$$


In adjustment by the conditional method, the conditional equation of the coplanarity of three vectors - the directions

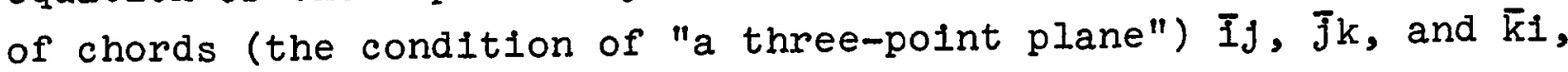
on the basis of $(20.5)$, is written as

$$
\begin{aligned}
& {\left[\frac{\partial F}{\partial I_{I j}}\left(\frac{\partial L}{\partial \Phi} \cdot \frac{\partial \Phi}{\partial h}+\frac{\partial L}{\partial \Lambda \cos \Phi} \cdot \frac{\partial \Lambda \cos \Phi}{\partial h}\right)+\right.} \\
& +\frac{\partial F}{\partial M_{i j}}\left(\frac{\partial M}{\partial \Phi} \cdot \frac{\partial \Phi}{\partial h}+\frac{\partial M}{\partial \Lambda \cos \Phi} \cdot \frac{\partial \Lambda \cos \Phi}{d h}\right)+ \\
& \left.+\frac{\partial F}{\partial N_{i j}}\left(\frac{\partial N}{\partial \Phi} \cdot \frac{\partial \Phi}{\partial h}+\frac{\partial N}{\partial \Lambda \cos \Phi} \cdot \frac{\partial \Lambda \cos \Phi}{\partial h}\right)\right] v_{h_{l j}}+ \\
& +\left[\frac{\partial F}{\partial L_{i j}}\left(\frac{\partial L}{\partial \Phi} \cdot \frac{\partial \Phi}{\partial a}+\frac{\partial L}{\partial \Lambda \cos \Phi} \cdot \frac{\partial \Lambda \cos \Phi}{\partial a}\right)+\right. \\
& +\frac{\partial F}{\partial M_{i i}}\left(\frac{\partial M}{\partial \Phi} \cdot \frac{\partial \Phi}{\partial a}+\frac{\partial M}{\partial \Lambda \cos \Phi} \cdot \frac{\partial \Lambda \cos \Phi}{\partial a}\right)+ \\
& \left.+\frac{\partial F}{\partial \Lambda}\left(\frac{\partial N}{\partial \Phi} \cdot \frac{\partial \Phi}{\partial a}+\frac{\partial N^{\prime}}{\partial \Lambda \cos \Phi} \cdot \frac{\partial \Lambda \cos \Phi}{\partial a}\right)\right] v_{a_{i j}}+ \\
& +\left[\frac{\partial F}{\partial L_{j k}}\left(\frac{\partial L}{\partial \Phi} \cdot \frac{\partial \Phi}{\partial h}+\frac{\partial L}{\partial \Lambda \cos \Phi} \cdot \frac{\partial \Lambda \cos \Phi}{\partial h}\right)+\right. \\
& +\frac{\partial F}{\partial M_{i k}}\left(\frac{\partial M}{\partial \Phi} \cdot \frac{\partial \Phi}{\partial h}+\frac{\partial M}{\partial \Lambda \cos \Phi} \cdot \frac{\partial \Lambda \cos \Phi}{\partial h}\right)+ \\
& \left.+\frac{\partial F}{\partial N_{j k}}\left(\frac{\partial \Lambda}{\partial \Phi} \cdot \frac{\partial \Phi)}{\partial h}+\frac{\partial N}{\partial \Lambda \cos \Phi} \cdot \frac{\partial \Lambda \cos \Phi}{\partial h}\right)\right] v_{k_{j_{k}}}+ \\
& \left.+\ldots+\frac{\partial F}{\partial N_{M}}\left(\frac{\partial N}{\partial \Phi} \cdot \frac{\partial \Phi}{\partial a}+\frac{\partial N}{\partial \Lambda \cos \Phi} \cdot \frac{\partial \Lambda \cos \Phi}{\partial a}\right)\right] v_{a_{k i}}+W_{F}=0 \text {; }
\end{aligned}
$$

where

$$
W_{F}=F^{0}-F^{\prime}
$$

$F^{0}$ is calculated from the preliminary coordinates of points 1 , $j$, and $k$.

$F^{\prime}$ is calculated from the direction cosines $L+d \Lambda, M+d M$, and $\mathrm{N}+\mathrm{dN}$, adjusted in the first stage, of each chord by using $(24.37)$ and $(24.39)$ in succession. 
25. Information about Adjustment of Space Geodetic Networks Created by the Orbital Method

Space triangulation is the basic, but not the only, method of creating space geodetic networks.

It is assumed in space triangulation that every AES position is not directly related to others. However, satellite motion is actually subject to specific laws. If at a given moment to a satellite is located at point $r_{0}\left(x_{0}, y_{0}, z_{0}\right)$ and has a velocity $v_{0}\left(\dot{x}_{0}, \dot{y}_{0}, \dot{z}_{0}\right)$, at the next moment $t_{k}$ it will be located at a quite specific point $r_{k}\left(x_{k}, y_{k}, z_{k}\right)$ and will have a velocity $v_{k}$ $\left(\dot{x}_{k}, \dot{y}_{k}, \dot{z}_{k}\right)$. Consequently, at any moment $t_{k}$ the coordinates and velocity of a satellite may be expressed by its coordinates and velocity at some initial moment to

$$
\begin{gathered}
r_{k}=v_{1}\left(x_{0}, y_{0}, z_{0}, \dot{x}_{0}, \dot{y}_{0}, \dot{z}_{0}, t_{k}\right), \\
v_{k}=v_{2}\left(x_{0}, y_{0}, z_{0}, \dot{x}_{0}, \dot{y}_{0}, \dot{z}_{0}, t_{k}\right) .
\end{gathered}
$$

For all measurements made at moments $t_{k}$ within the interval $/ 101$ $\Delta t$, we may compile equations which connect the coordinates of observation points 1 and the running coordinates and components of the velocity vector of the AES. These equations are presented in Section 15 for measured values of $\alpha_{i k}, \delta_{i k}$, and $\rho_{i k}$. The equation for $\Delta \rho$ is also presented there, but in the orbital method this measurement may be replaced by the radial component of AES velocity $-\dot{\rho}_{1 k}$. To derive the equation linking $\dot{\rho}$ to the unknowns - the point coordinates $\left(X_{1}, Y_{1}, Z_{1}\right)$ and the orbital parameters $\left(x_{k}, y_{k}, z_{k}, \dot{x}_{k}, \dot{y}_{k}, \dot{z}_{k}\right)$, it is sufficient to use the relation which ensues from Figure 39, 


$$
\ddot{\rho}_{i k}=v_{k} \cos \beta
$$

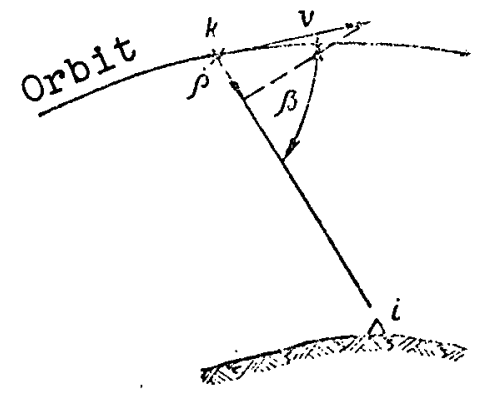

Figure 39.
Taking into account the fact that

$$
\cos \beta=\frac{1}{v_{k} \rho_{l k}}\left(\Delta X_{i k} \dot{x}_{k}+\Delta Y_{l k} \dot{y}_{k}+\Delta Z_{i k_{k}} \dot{z}_{k}\right)
$$

we will have

$$
\dot{\rho}_{i k}=\frac{1}{\rho_{l k}}\left(\Delta X_{l k} \dot{x}_{k}+\Delta Y_{i k} \dot{y}_{k}+\Delta Z_{i k} \dot{z}_{k}\right)
$$

Linearization of the observation

equations leads to the correction equations

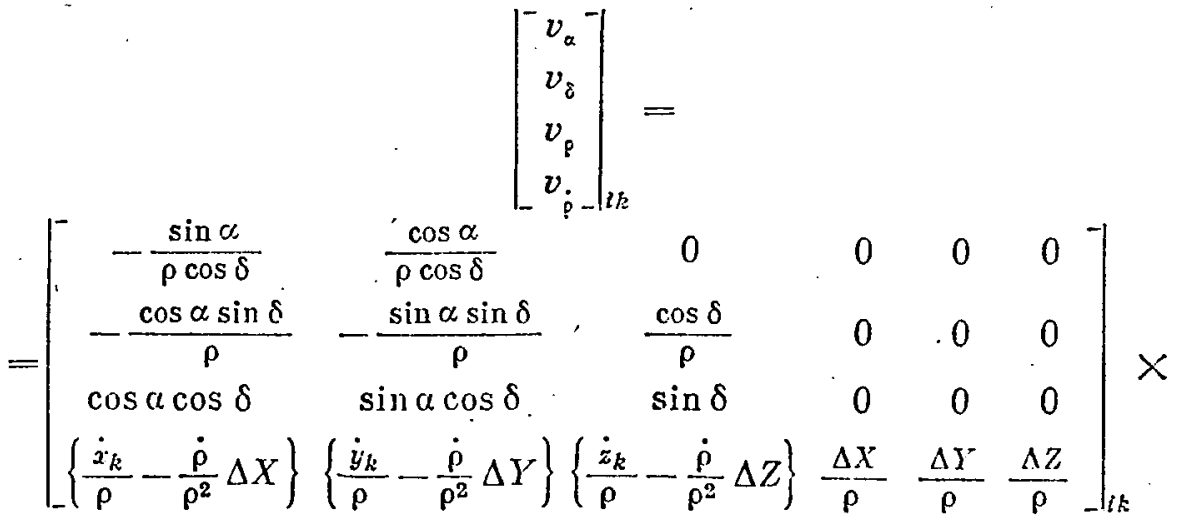

$$
\begin{aligned}
& \times\left[\begin{array}{c}
d\left(x_{k}-X_{i}\right) \\
d\left(y_{k}-Y_{i}\right) \\
d\left(\tilde{z}_{k}-Z_{i}\right) \\
d \dot{x_{k}} \\
d \dot{y_{k}} \\
d \dot{\dot{z}_{k}}
\end{array}\right]+-\left[\begin{array}{c}
l_{\alpha}^{-} \\
l_{\delta} \\
l_{p} \\
l_{\dot{p}}
\end{array}\right]_{i k}
\end{aligned}
$$

or in reduced notation

$$
\begin{aligned}
v= & \frac{\partial(\alpha, \delta,}{\partial(x, y, z, \dot{\rho})_{i k}} d \dot{\dot{x}, \dot{y}, \dot{z})_{k}} d(x, y, z, \dot{x}, \dot{y}, \dot{z})_{k-}^{\prime}- \\
& -\frac{\partial(\alpha, \delta, \rho, \dot{\rho})_{i k}}{\partial(X, Y, Z)_{i}} d(X ; Y, Z)_{i}^{\prime}+L .
\end{aligned}
$$


The weights of correction Equations (25.4) are established according to the measurement accuracy.

Reducing Equations (25.1) to linear form and substituting the result in.(25.4), we obtain

$$
\begin{gathered}
v=\frac{\partial(\alpha, \delta, \rho, \dot{\rho})_{i k}}{\partial(x, y, z, \dot{x}, \dot{y}, \dot{z})_{k}} \cdot \frac{\partial(x, y, z, \dot{x}, \dot{y}, \dot{z})_{k}}{\partial(x, y, z, \dot{x}, \dot{y}, \dot{z})_{0}} \times \\
\times d(x, y, z, \dot{x}, \dot{y}, \dot{z})_{0}^{\prime}-\frac{\partial(\alpha, \delta, \rho, \dot{\rho})_{i k}}{\partial(X, Y, Z)_{i}} \cdot d(X, Y, Z)_{i}^{\prime}+ \\
+L==Q R d(x, y, z, \dot{x}, \dot{y}, \dot{z})_{0}^{\prime}-S d(X, Y, Z)_{i}^{\prime}+L .
\end{gathered}
$$

Essentially, introduction of Equations (25.1) and the transition from correction Equations (25.4) to (25.5) indicates conversion from the space triangulation method to the orbital method of constructing space geodetic networks. In the orbital method, as can be seen from Equations (25.5), corrections for some elements which characterize the entire orbital arc in the assumed time interval $\Delta t$ are sought rather than corrections for the coordinates of individual AES positions. Depending on the extent and distribution of the measurements, and the accuracy of the precalculations of the AES positions (the accuracy of numerical integration of the equations of motion), the values of the refined arcs may be different.

A group of correction Equations (25.5), to which corresponds a partially independent system of six normal equations, is complled for each arc according to the number of measurements. The form of the upper right side of the general symmetric matrix of the coefficients of the normal equations, which occur in the orbital method, is shown in Figure 40. This matrix, as in adjustment of space triangulation by the parametric method, is 1deal for solution of the system according to the Pranis-Pranevich method. 


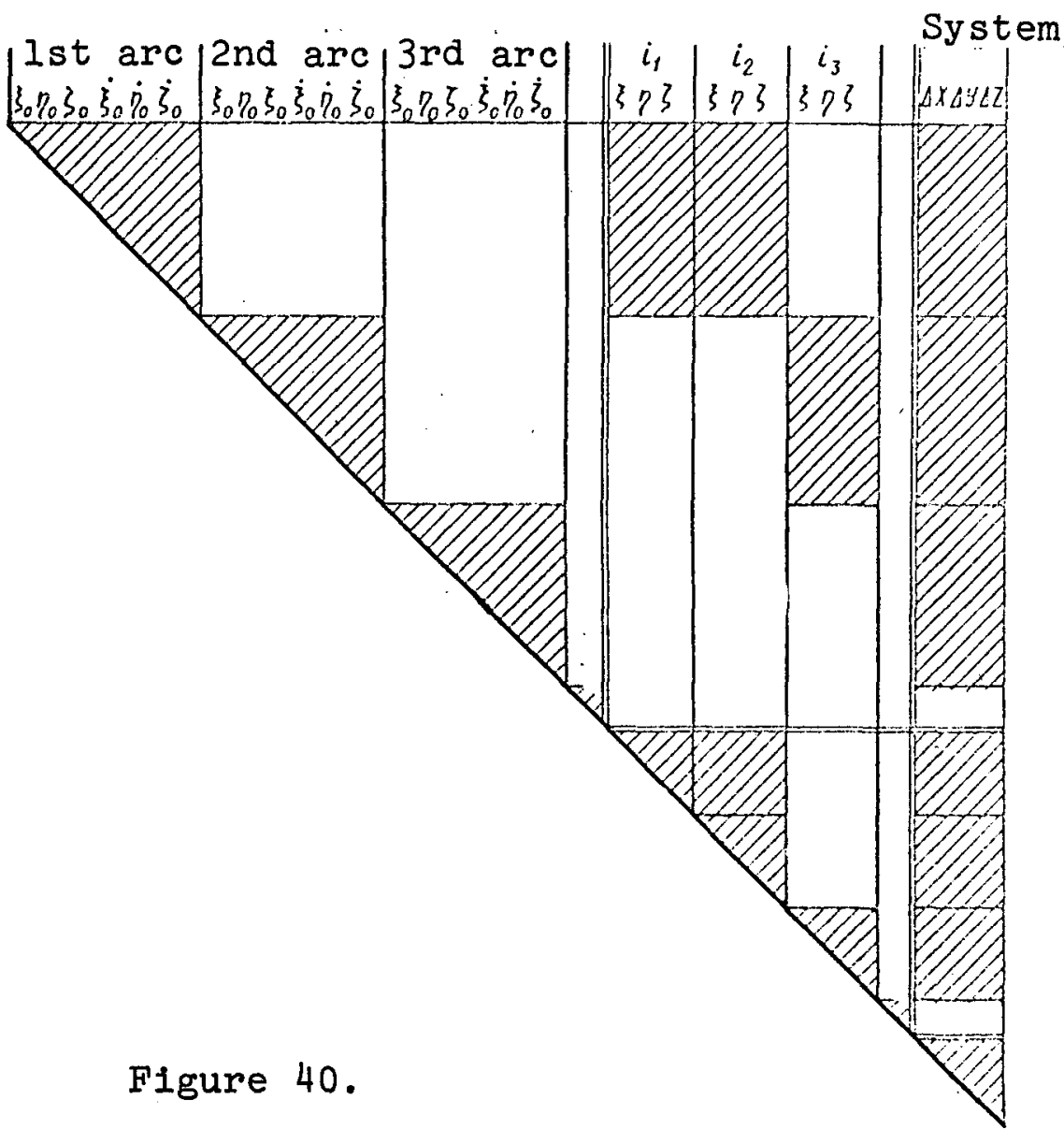

The orbital method, as already mentioned, makes it possible not only to calculate the mutual position of observation points, but to relate the origin of the coordinate system to the Earth's center of mass. Therefore, in adjustment of a network constructed by this method, along with correction of the coordinates of Individual observation points, we can calculate the corrections common to all observation points by conversion of the coordinate system origin.

The initial equations of the orbital method are those Equations (25.1) which we formally introduced. Actually, obtainIng these equations is a very complex matter and is one of the 
important branches of celestial mechanics. The motion of a satellite in the general case is described by a system of three differential second-order equations

$$
\begin{aligned}
& \ddot{x}==\frac{\partial V}{\partial x}+\sum Q_{x} \\
& \ddot{y}=-\frac{\partial V}{\partial y}+\sum_{y} Q_{V} \\
& \ddot{z}=\frac{\partial V}{\partial z}+\sum Q_{z},
\end{aligned}
$$

where $\partial \mathrm{V} / \partial \mathrm{x}, \partial \mathrm{V} / \partial \mathrm{y}$, and $\partial \mathrm{V} / \partial \mathrm{z}$ are the components of potential forces; and $Q_{x}, Q_{y}$, and $Q_{z}$ are the components of forces having no potentials.

A large number of forces act on AES motion. These are primarily the gravitational forces of the Earth, Moon, and Sun, atmospheric drag, light pressure, etc. Rigorous integration of Equations (25.6) with consideration of all effective forces is impossible. Therefore, in all cases approximate methods of their integration must be used.

As can be seen from Formulas (25.5), Integration of the equations of motion is required for two purposes: first, to obtain the free terms of the correction equations, and secondly, to calculate the coefficients of matrix $R$.

It is natural that, in calculating the free terms, the errors committed in integration of the equations of motion will lead to methodical errors in the solution; therefore, in the given case the most accurate of the known methods of integration must be used, taking into account all known perturbing forces. It is now possible, due to the use of high-speed electronic computers, to employ numerical methods (for example, Runge-Kutta or Adams methods), which make it possible to obtain more accurate solution of Equations (25.6). 
Using numerical integration of the equations of motion (25.6), we can calculate the matrix of the derivatives of $R$ as well. For this we must attach importance to the increments of the initial conditions and to the numerical difference method to obtain the values of the derivatives for the running moments of measurements of $t_{k}$, i.e., a total of 12 matrices must be calculated of the form

$$
\left[\frac{\Delta x_{k}}{\sigma_{i}} ; \frac{\Delta y_{k}}{\sigma_{i}} ; \frac{\Delta z_{k}}{\sigma_{i}} ; \frac{\Delta \dot{x}_{k}}{\sigma_{i}} ; \frac{\Delta \dot{y_{k}}}{\sigma_{i}} ; \frac{\Delta \dot{z}_{k}}{\sigma_{l}}\right]
$$

where $\sigma$ are the variations of the initial conditions to which must be added in sequence the values of $\pm \Delta \mathrm{x}_{0}, \pm \Delta \mathrm{y}_{0}, \pm \Delta \mathrm{z}_{0}, \pm \Delta \dot{\mathrm{x}}_{0}, \pm \Delta \dot{\mathrm{y}}_{0}$, and $\pm \Delta \dot{z}_{0}$. It is natural that this method of calculating the derivatives requires additional twelve-fold integration of Equations (25.6). Even with modern computers this entails significant expenditures of time.

Taking the fact into account that the coefficients of matrix $R$ may be known approximately, we may calculate only the main effective force in Equations (25.6), for example, by assuming that $V=f M / r$ and $Q=0$. In this case the equations of motion 'may be integrated precisely and the analytical expressions in the form of (25.1) may be obtained for the coordinates and the velocity components of the AES as a function of the six integration constants. By differentiation of these expressions, we may obtain the analytical expressions for the derivatives of matrix R. The coefficients of matrix $R$ obtained in this way may be found in Charnyy's work [51]. Several more rigorous formulas which take into account the linear (with respect to time) secular variations of the orbital elements are presented in Kaula's work: [26]. 
The orbital method has three main advantages compared to the space triangulation method:

- the number of parameters to be calculated is reduced;

- synchronization of observations is unnecessary, and the solution includes both simultaneous and non-simultaneous measurements, which makes it possible to increase their total number;

- It becomes possible to relate the origin of the coordin- 1105 ates to the Earth's center of mass.

However, this method has one considerable disadvantage which limits its use at present. In order to use this method, it is necessary to know with high accuracy all the forces affecting the AES motion.

Obviously, the best solution of geometric problems of space geodesy in the future may be provided by joint use of the orbital and space triangulation methods.

\section{Comparative Survey of Methods of Space} Triangulation Adjustment

The classification of space triangulation adjustment methods is based on their different features. Some methods permit joint adjustment of all observations, and others make it possible to divide adjustment into stages. In some methods, the coordinates of all points of the network, including the AES positions, are used as unknowns, and in others only the coordinates of ground points are used. There are methods based on the parametric method of adjustment and other methods use the conditional method and variations of it. Specific combinations of the different methods are also possible. 
For subsequent comparative analysis of the advantages and disadvantages of these methods, we feel it is expedient to divide adjustment into stages as the basis of classification, which will correspond to a specific extent to the different methods of constructing space triangulation. A diagram of such a classification is presented in Figure 41.

The problem of selecting the space triangulation adjustment method is solved by the analysis of a number of factors. The main ones include the form of the measured geodetic data (only photographic observations or their combinations with linear observations), the rigorousness of the adjustment method, the nature of the construction of the net (free or bound), the predominant composition of the groups, the volume of computer operations, and the complexity of compiling programs for calculations on electronic computers. Let us dwell in more detail on some of these factors.

A rigorous solution of the adjustment problem by the least squares method is based on the following assumptions:

- the observations contain only random errors;

- a weight, inversely proportional to the dispersion value, is attributed to each measurement;

- adjustment is carried out provided that $[\mathrm{pvv}]=\min$, where $v_{i}$ are corrections of directly measured values.

Measurements of the elements of space networks will entail inevitable errors whose nature and extent depend on the type of equipment, its analytical capability and reliability, the organi-/107 zation of simultaneous observations, the methods of preliminary processing, etc. 


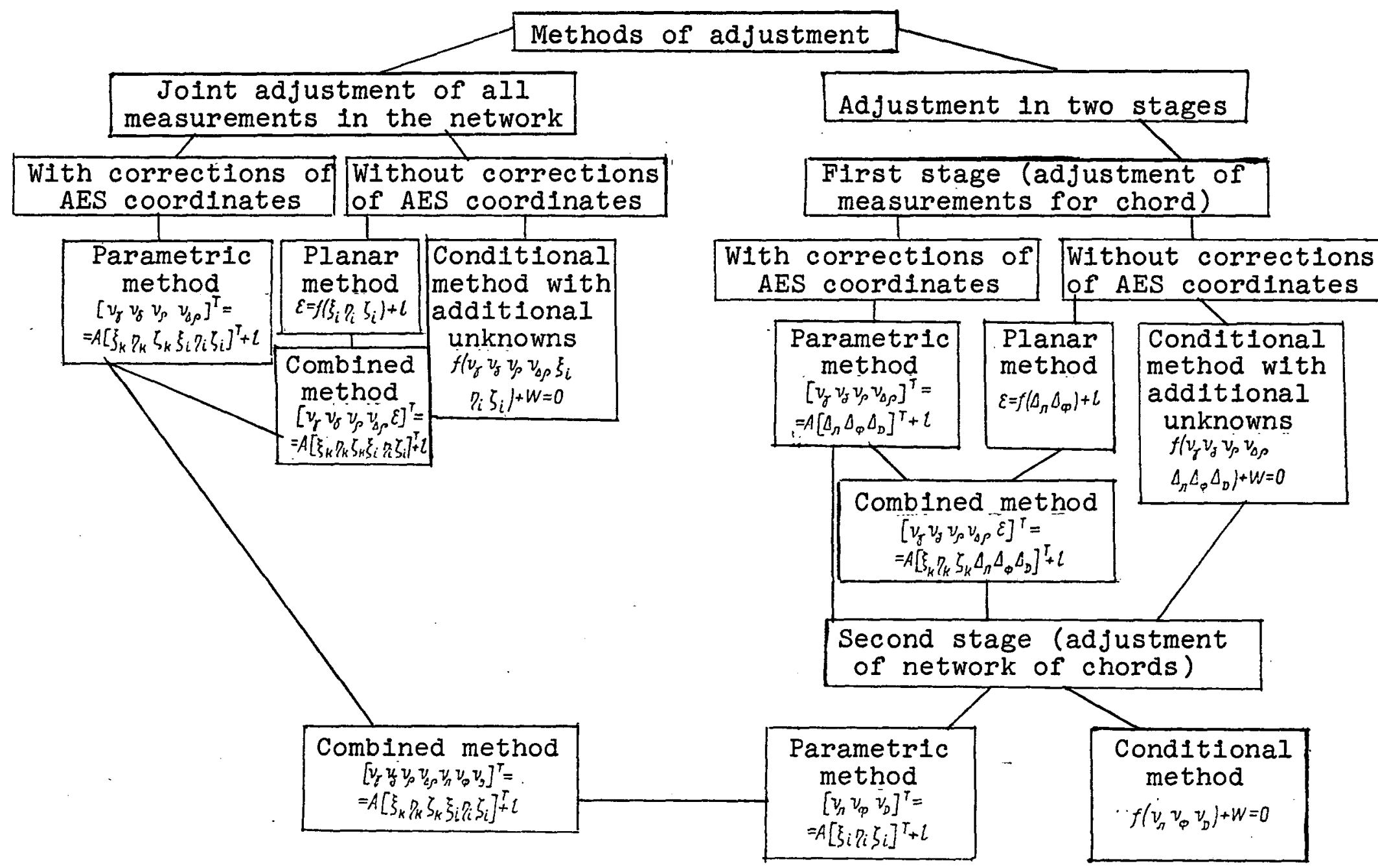

Figure 41. 
Due to the complexity and variety of equipment used, as well as the enormous scale of investigations on development of space geodetic networks, the number of possible sources of errors is so great that the most careful investigations can hardly conceal, and even less eliminate, the resulting errors. Therefore, the probability of systematic errors in space triangulation is increased compared to ordinary geodetic networks.

In many cases, the variance or variance ratio for different measured values remains unknown, which undoubtedly makes it difficult to determine the system of weights correctly.

Thus, even the first two conditions of a rigorous solution of the problem of adjustment in the development of space geodetic networks by the least squares method may be disturbed to some extent.

This, of course, should not orient geodesists to the use of approximate methods of adjusting space networks. Depending on the depth of our knowledge, many sources of systematic errors will be eliminated, and then the rigorousness of mathematical treatment will begin to acquire even greater significance.

Joint adjustment of all observations of space triangulation by the parametric method and by the conditional method with additional unknowns is rigorous for networks of any construction.

Adjustment of the planes is applicable only to networks constructed from simultaneous photographic observations. Moreover, this method will be rigorous if each AES position is observed from two points only. 
The situation is similar when using the two-stage method of adjusting three-dimensional triangulation constructed from chords. If the observation of AES positions, contained in simultaneous groups, consisting of three or more directions, are used to calculate the geometric elements of the chords, the rigorous nature of the method is disturbed.

However, the number of such groups and the relationship to the total number of measurements must be taken into account. Failure to consider single functions makes it difficult to determine the welghts, whereas rejection of a large number of relationships may lead to a loss of accuracy.

Adjustment of space triangulation constructed from chords in the second stage of the two-stage method of adjustment will be rigorous if the generalized condition of the least squares method is used.

However, for example, this method may be used for a network of chords with well developed technology and computer programs for adjustment of the entire space triangulation by the parametric method. In this case, its use will not be rigorous if the diagonal coefficients instead of complete correlation matrices for each chord, are used as the weights of the chord elements. The correctness of assuming it is not rigorous will depend on the purposes of adjustment, the measurement accuracy and the desired results. In each specific case of using approximate weighting matrices, the anticipated methodical errors should be previously calculated theoretically or from calculations on the models of the networks. 
The volume of calculating operations depends on the method of adjustment. This problem cannot be reduced only to determining the order of the matrix of normal equations, which originate with the conditional method or the parametric method. The simplicity of the algorithms, the cyclicity of operations, and the possibility of standardization of calculating procedures are of great Importance.

Let us consider space triangulation adjustment methods from this point of view.

The preliminary coordinates of the AES positions must be calculated in adjustment of all observations of the network or of observations of each chord in the first stage of adjustment of triangulation, constructed from chords, by the parametric method. Calculation of the preliminary coordinates of AES positions is not required in adjustment of all measurements of the network by the conditional method with additional unknowns, as well as during the first stage of adjustment of a network constructed of chords, if the latter are obtained only from photographic observations.

The form of the correction equations (measured values of $\gamma$ and $\delta$ ) is simple and standard in adjustment by the parametric method.

The form of the conditional equations in the conditional method with additional unknowns is more complex, but also standard, if we remember that all conditions are described by the conditional equations of the synchronization planes, and the conditions of "fixed" lengths and directions of the chords are described by the corrections of these "fixed" values. The calculations in writing correction equations for the synchronization 
planes do not differ in any way from those for conditional equations with additional unknowns, because the weights of the equations, which are functions of the coefficients in the case of corrections of measured values, must be calculated in addition to the coefficients of equations in the case of triangulation unknowns.

In order to compare the volume of calculations in the different methods, the number of arithmetic operations for each of them could be calculated. However, such a detailed comparison has hardly any practical value due to the two following factors. It was noted above that real measurement accuracy cannot be judged by the free terms of the conditional equations of the synchronization planes, since their value depends on the assumed values of the preliminary coordinates of the observation points. Nevertheless, calculation of the free terms of these equations, as indicated by practice, is an obligatory process of preliminary calculations, because it makes it possible to reveal crude errors in observations and especially errors in their synchronization. In view of this, the volume of preliminary calculations, including calculation of the preliminary AES coordinates and the points, the free terms of the conditional equations of ail synchronization planes and of the coefficlents of the equations, will be approximately the same for the different adjustment methods. The second factor is the fact that adjustment of space triangulation at present can hardly be assumed without the use of universal computers. Therefore, the slight deviations in the order and extent of preliminary calculations for the different methods of adjusting triangulation networks constructed only from simultaneous photographic observations may not be considered as significant. 
The adjustment of space triangulation networks, constructed from combinations of photographic observations with measured distances and the differences in distances to the AES, is different. The equations for correction of the measured distances and the differences in distances are simple and the order of their compilation is standard in the parametric method of adjustment.

The conditional method with additional unknowns is considerably inferior in this regard to the parametric method of adjustment. We are not only concerned with the very complex form of the formulas from which the coefflcients and free terms of the conditional equations are calculated. The main difficulty consists in the geometric "dissimilarity" of the figures of such networks. Any section of a network of specific dimensions may differ from another both in the number of conditions and in their form, as well as in the number of unknowns contained in the initial conditional equations. For this reason it is much more difficult to organize calculations by the conditional method with additional unknowns within the limits of a single computer program than in the parametric method. This will also be valid in adjustment of three-dimensional triangulation from chords. However, in the latter case the conditional method will make it possible to estimate the accuracy of calculating the geometric elements of the network from the residuals of the conditional equations.

The problem of the order of the systems of normal equations, solved jointly by a certain method, is of definite importance for comparative analysis of adjustment methods.

Data"were presented above on the number of normal equations, solved in each method. 
For simultaneous adjustment of all triangulation measurements, the number of normal equations will be $N=3 s+3 P$ in the parametric method and in the conditional method with additional unknowns, $\mathrm{N}=\sum_{s=1}^{s}(2 n-3)+r+3 \%$, where $\mathrm{s}$ is the number of AES positions; $P$ is the number of points; $r$ is the number of linear measurements; and $\mathrm{n}$ is the number of directions to the position of each AES.

It is easy to see that, if a three-dimensional network is constructed only from the measured directions which form synchronous pairs $(n=2)$, the number of normal equations in the parametric method of adjustment is greater by $2 \mathrm{~s}$ than in the conditional method.

In synchronous groups consisting of three directions, the number of normal equations is identical, and with a greater number of directions in synchronous groups the number of correlates exceeds the number of corrections of the coordinates of AES positions. Addition of linear measurements to photographic observations increases the order of the systems of normal equations even more in the conditional method with additional unknowns.

A very large number of AES positions may be observed over a prolonged period of observations for construction of space triangulation. Simultaneous adjustment of the results of all observations may lead to a system of normal equations of very high order (several thousands). Even the use of modern computers requires a long time of continuous operation for solution of such a system. The use of a two-stage adjustment method makes it possible to divide this process into solution of independent systems of normal equations whose number will be equal to the number of chords in the network. Moreover, systems of only two normal equations will be solved in the first stage in the planar method. 
The system of normal equations in the second stage of adjusting triangulation from chords in the case of the parametric method will have an order of $N=3 P$. The use of the method of conditional measurements in the second stage requires solution of a system of normal correlate equations of order $N=2 n-3 P+$ $3 \mathrm{P}_{0}+\mathrm{B}$ for a network of the directions of chords and of order $N=3 n-3 P+3 P_{0}+B$ for a network of the directions and bases of chords. (It is assumed in the formulas that $\mathrm{n}$ is the number of chords, $P$ is the number of observation points to be calculated, $P_{0}$ is the number of initial observation points, and $B$ is the number of "fixed bases.)

The expression for $\mathrm{N}$ indicate that in a network constructed from the directions of the chords, the number of normal correlate equations will be less than the number of normal equations for correcting the coordinates, if there are no diagonal chords in the triangulation (by analogy with ordinary triangulation, there are no overlapping triangles). In the presence of diagonal chords and "measured" bases of the chords, the system of equations in the parametric method will be smaller than in the conditional method.

As a result of comparing the advantages and disadvantages of adjustment methods (Figure 4I), we can make some recommendations on their use for adjusting triangulation networks of different construction.

Adjustment of space triangulation by the conditional method with additional unknowns is generally feasible, especially for networks constructed by a combination of photographic and range measurements. 
Adjustment of space triangulation with relatively small distances between observation points (small chord bases), when most AES positions are observed from more than two observation points, is feasible by the parametric method.

Adjustment of space triangulation with large chord bases, when most AES positions are observed from two observation points and when there are only photographic observations, is feasible by the plane method. If a very large number of AES positions is observed in such triangulation, and also if the observation data are gradually accumulated in time, first for some chords and then for others, adjustment in two stages may be used. In this case, the parametric method is more convenient in both the first and second stages. 


\section{CHAPTER 6}

ERRORS IN THE POSITION OF POINTS IN SPACE TRIANGULATION

27. The Importance and the Problems of a Priori Analysis of the Accuracy of Space Triangulation

A priori investigation of the error distribution in geodetic networks is the theoretical foundation for solving an entire group of problems of geodesy, such as development of schematic diagrams for construction of geodetic networks, drafing designs and their evaluation, determination of the optimum forms of geodetic structures, and development of the technical requirements for the optimum values of the elements (angles and sides) of geodetic networks. All these problems have always been at the center of attention of geodesists and their investigation for ordinary geodetic networks has been continued up to the present time. These problems are even more timely for space geodetic networks.

We may assume that a priori evaluation of the accuracy of space triangulation structures follows two goals:

- determination of the anticipated accuracy of a specific design (comparison of the accuracy of several designs) of a space triangulation network;

- comparative analysis of different schemes of constructing space triangulation for purposes of selecting the optimum types and optimum dimensions of the geometric elements and figures for use in the design of space triangulation networks. 
We note that in all cases the basic criterion of accuracy is the error in the position of the triangulation point.

It was mentioned above that space triangulation has no direct relationships (measurements) in it between observation points, but the instantaneous positions of the satellite are the intermediate points. In this regard, the number of measured values, connecting the AES position, is considerably less than the number of measurements connecting the ground point to the AES positions, observed from a given point over a prolonged period of time. Therefore, we should assume that the same requirements should be placed on analysis of the accuracy of AES positions as on the individual figures of space triangulation.

We feel that it is more convenlent to obtain expressions 1113 which are rather simple and descriptive, even if this requires specific simplification of rigorous, but more complex formulas due to a decrease in the accuracy of the results obtained, for a priorl analysis of the accuracy of the position of space triangulation points.

It is expedient to place requirements on the expressions for the error in the position of points of individual figures, so that they are invariant with respect to the transformation of the coordinate systems and contain only the parameters which characterize the "internal" geometry of these figures.

For figures forming a series of space triangulation, it is desirable to investigate the dependence of the position error on the form of the figure. 
The expression for the error of the point of a space triangulation series should reflect the dependence of the error increment on the increase in the length of the series, i.e., the nature of the accumulation of errors.

Expressions for errors in the position of the points of a continuous space triangulation network should indicate the dependence of the errors on the distances from the initial points, as well as on the average values of the distances between points.

In the general case, the mean square error of the given function of measured values is described by the tensor

$$
M=F \%
$$

where $F$ is the vector of the partial derivatives of the given function, $\mathbb{M}^{2}$ is the correlation matrix of the errors in the arguments of the function. For independent arguments, $M^{2}$ is transformed into a diagonal matrix.

For adjusted values of the arguments, Expression (27.1) assumes the form

$$
M A=P(1,0) P^{\prime \prime}
$$

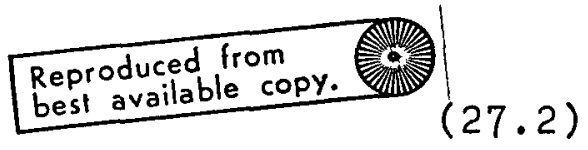

where $\mu$ is the error of unit weight; and $Q$ is the reciprocal matrix of the system of normal equations.

Tensor (27.I) is used to the full extent for a posteriori analysis of accuracy, possible after adjustment of space triangulation.

For a priori analysis of accuracy, the elements of tensor (27.1) are obtained with a greater or lesser degree of approximation. 
The error in the unit weight depends primarily on the value of the free terms of the coupling equations between the measured values and the unknowns. In view of this, it expresses the effect of measurement errors. Moreover, in cases when the initial data contain errors which are commensurable with measurement errors, and the amount of initial data and their distribution in the triangulation network is such that they affect the value of the free terms of the equations, the error of unit weight includes the errors of the initial data.

The elements of the reciprocal matrix of the coefficients 1114 of the normal equations and of matrix $F$ depend on the value of the coefficients of the coupling equations between the measured values and the unknowns and on the number of equations. Because of this, they reflect the effect of the geometric shape of space triangulation construction and the number of measurements.

Thus, an error in the function of the adjusted unknowns depends on:

- measurement errors,

- the errors in the initial data,

- the geometric construction of triangulation,

- the number of measurements.

Design of space triangulation is usually based on the fact that observations will be carried out by equipment which provides a given accuracy. In this regard, measurement errors are assumed to be the knowns for a priori analysis of accuracy. However, a certain dependence between measurement accuracy and the geometric shape of the triangulation figures, which has an opposite effect on the results of accuracy analysis, may exist. Thus, for 
example, the refraction effects in photographic observations of AES reduce measurement accuracy at low AES elevation above the horizon of the observation point, whereas (as will be shown below) it is preferable to observe the AES at minimum elevation with respect to the optimum geometric shape of the figure from which the direction of the chord between two points is calculated.

The effect of errors in the initial data on the results of a priori accuracy analysis must be taken into account to a considerably greater extent than is permitted by a posterior 1 accuracy analysis.

Errors of initial data, when the latter number is small, are often of a systematic nature (error in the triangulation scale, the total shift or rotation of the network) and are not reflected in the value of the free terms of the equations, and consequently, do not appear in a posteriori analysis. The formulas of a priori accuracy analysis should also reflect such influences. Moreover, In many cases the formulas of a priori analysis of accuracy of space triangulation will be based on sequential analysis of the accuracy of the measurement function due to the complexity of the figures of space triangulation and its specific features, included in sequential calculation of first the AES positions, and then of the observation points. In this case, the errors in the results of each preceding construction emerge as the errors in initial data for a subsequent construction, and their effect on the final result must be taken into account.

The most important factor in a priori analysis of accuracy is the consideration of the effect of the geometric characteristics of space triangulation construction, because the essence of the design of space triangulation networks is also included, as was mentioned above, in a determination of the optimum mutual 
position of the observation points and of the AES positions to be observed. Therefore, main attention in this chapter is devoted $/ 115$ to selecting the formulas for a priori analysis of the accuracy of the elements and figures of space triangulation, which reflect the effect of the geometric shape of its structure.

An increase in the number of measurements increases the weight of the function of the adjusted values of space triangulation. However, this increase should be regarded as comparable to the required number of measurements to calculate the unknowns. An increase in redundant measurements when retaining the geometric shape of a triangulation structure increases the welght in proportion to this increase. The redundant measurements, which alter the structural shape, are reflected in the accuracy of the results in a more complex manner.

An inverse dependence, caused by the specific nature of the observation of space objects, may also exist between the effect of the number of measurements and the shape of the triangulation structure on the accuracy of the results, besides this dependence. It includes the fact that the probability of an AES passing through positions which are optimum with respect to the shape of the triangulation structure may be considerably less than that of passage through a more extensive, but geometrically less optimum zone of the celestial sphere. Therefore, in the given limited AES observation period, for example, the requirements on the number of observations may prevail over those on the optimum geometric shapes of the structures, to achieve a specific accuracy of results.

Space triangulation in any method of construction consists of individual figures; therefore, the method of estimating the accuracy of the points obtained from the individual figures of space triangulation will be primarily discussed below. 
The expressions for approximate a priori analysis of the accuracy of elementary figures will be considered below. The effect of errors in the initial data and redundant measurements on the results of the accuracy analysis will then be investigated separately.

In conclusion, the problems of a priori analysis of the accuracy of the positions of the points of series and of continuous space triangulation networks, formed by the corresponding figures, will be discussed.

\section{Methods of A Priori Accuracy Analysis}

A priori determination of the elements of the error tensor of the function of measured or adjusted values of (27.1) and (27.2), and especially their analytical representation as a function of the perturbing factors, entails considerable difficulties. Hence, approximate and stochastic methods of analysis have become widespread. Let us present a somewhat more detailed description of the possible methods of a priori analysis of accuracy. They will depend on the complexity of the object being studied (the individual element, figure, series or network) and the permissible degree of approximation of the formulas. For example, the errors in such elements of a space triangulation network as the direction toward the AES, the synchronization plane, the chord, and the apexes of the elementary figures may be deter- 1116 mined mined by using the general rules and procedures of the theory of measurement errors. The estimates obtained should have been rigorous, but due to the complex form of the functions, they had hardly any practical value. Moreover, the nature of the problem itself is such that it is more important to obtain approximate estimates of simple form, rather than rigorous but cumbersome formulas, taking into account their future engineering use. 
It is expedient to use vector algebra - 1.e., to consider the error in the position of a point or in the shift of a line as some random vector in three-dimensional space - to derive the formulas of approximate analysis of the accuracy of constructing the individual elements and figures of space triangulation. This usually simplifies the derivation of formulas considerably and gives them a descriptive geometric meaning. Moreover, vector representation makes it possible to obtain formulas for the accuracy analysis which are invariant with respect to coordinate transformations, 1.e., to express them by the parameters which characterize the "Internal" geometry of the figures of space triangulation (1.e., by the angles and sides of the given figure).

Geometric interpretation of the errors in the elements of space triangulation provides a certain simplicity and descriptiveness to the expressions of a priori accuracy analysis. In this case, we start with the fact that each measurement determines some position surface in space, 1.e., the surface on which the observation point or the AES position to be calculated is located. Thus, for example, the measured values of space triangulation determine the position surface: $\delta$ is a cone, $\gamma$ is a plane, $\rho$ is a sphere, and $\Delta \rho$ is a hyperboloid.

Every measurement error leads to linear displacement of the corresponding position surface from the true position of the point to be calculated by a value

$$
\varepsilon=-\frac{m_{q}}{|\operatorname{grad} q|}
$$

where $\mathrm{m}_{\mathrm{q}}$ is the error in the measured value $q$,

$$
|\operatorname{grad} q|==\sqrt{\left(\frac{d q}{d x}\right)^{2}+\left(\frac{d q}{d y}\right)^{2}+\left(\frac{d q}{d z}\right)^{2}}
$$


Accordingly, when deriving formulas for a priori accuracy analysis, both direct measurement errors and displacements of the corresponding position surfaces may be used.

In adjustment of triangulation, the coupling equations of measurements with unknowns are linearized and, consequently, each position surface near the point to be calculated is replaced by a tangential plane. Approximation of any position surface by a tangential plane may also be used for a priori analysis of accuracy.

The use of geometric interpretation is especially effective for determining the effect of errors in initial data, because calculation of the coefficients of the reciprocal matrix of a system of normal equations prevents this effect from being taken into account.

Similar analyses for continuous space triangulation networks are more difficult to obtain due to the large number of connections between points and the complexity of the functional relationships.

The method of investigating accuracy with the aid of stochastic models of geodetic networks is used extensively at present. This method became possible through extensive introduction of electronic computers into geodetic analysis. The undoubted advantage of the modelling method is the possibility of determining the effect of different factors and thus of investigating them independently of each other, as well as the fact that an accurate value of Tensor (27.2) is obtained in these methods. However, this method has a number of disadvantages: each model of the geodetic network is a random sample - one of 
the infinite number of possible states of the given system after distortion of it. Therefore, the measurements of several tens of different variants of the distribution of measurement errors must be tested for the same model with the same mean square measurement error to obtain reliable estimates. This creates specific difficulties for any theoretical generalizations about the data of distorted model tests.

A method which is sometimes used to characterize the accuracy of the measured elements of networks after adjustment is the use of the theorem of the mean ratio of welghts which is expressed by the formula:

$$
\frac{p_{\text {adj }}}{p_{\text {meas }}}=\frac{n}{k}
$$

where $n$ is the number of all measurements; and $k$ is the number of required measurements. However, the estimate obtained according to this theorem will be too general.

The theorem of the mean ratio of weights is insensitive to the form of geodetic data. For example, the same numerical characteristics will be obtained for a network with measured angular values and distances as for a network with the same number of angular values and differences in distances. In fact, even averaged indicators of the accuracy of these networks will be different. Thus, the use of the theorem of the mean ratio of weights is justified only for comparison of the accuracy of networks, obtained on the basis of homogeneous geodetic data.

The most complete analysis of the accuracy of the elements of geodetic networks, as can be seen from (27.2), is carried out with the aid of the reciprocal matrix of the system of normal equations. 
As seen from Formulas (27.1) and (27.2), the reciprocal matrix operation must be carried out to analyze the accuracy of the different elements of a network, which is a difficult problem.

The problem may be simplified somewhat if a special coordin- /118 ate system in n-dimensional space is introduced in which the matrix of the coefficients of normal equations has an especially simple form. As we know from linear algebra, any symmetric matrix may lead to a diagonal form, 1.e., we must find a matrix* $D$ such that

$$
D F D^{-1}=\Lambda=\left[\begin{array}{cccc}
\lambda_{1} & 0 & \ldots & 0 \\
0 & \lambda_{2} & \ldots & 0 \\
\cdot & \ddots & \ddots & 0 \\
0 & 0 & \ldots & \lambda_{k}
\end{array}\right]
$$

where the values $\lambda_{1}, \ldots, \lambda_{k}$ are the eigenvalues of matrix $F$.

A matrix reciprocal to $\Lambda$ will also be a diagonal matrix

$$
\Lambda^{-1}=\left[\begin{array}{cccc}
\frac{1}{\lambda_{1}} & 0 & \ldots & 0 \\
0 & \frac{1}{\lambda_{2}} & \ldots & 0 \\
\ldots & \cdots & . & . \\
0 & 0 & \ldots & \frac{1}{\lambda_{k}}
\end{array}\right]
$$

It is easy to note that the analysis of accuracy for diagonal matrices is simplified to the maximum extent.

In practice, we must be concerned with arbitrary matrices. Therefore, we must consider the relationship of the elgenvalues of the matrix of the coefficients of normal equations to the welght coefficlents.

*D is the matrix of transition from an arbitrary base to one comprised of elgenvectors. 
The eigenvalues of matrices have a descriptive geometric meaning. Let us set a matrix of the coefficients of three normal equations

$$
F=\left[\begin{array}{lll}
f_{11} & f_{12} & f_{13} \\
f_{21} & f_{22} & f_{23} \\
f_{31} & f_{32} & f_{33}
\end{array}\right]
$$

and a reciprocal matrix

$$
F^{-1}=Q=\left[\begin{array}{lll}
q_{11} & q_{12} & q_{13} \\
q_{21} & q_{22} & q_{23} \\
q_{31} & q_{32} & q_{33}
\end{array}\right]
$$

The elements of matrix (28.6) may be regarded as the coefficients of the equation of an ellipsold, arbitrarlly located with respect to the coordinate axes. For example, the following /119 ellipsoid corresponds to matrix (28.6)

$$
\left[\begin{array}{lll}
x & y & z
\end{array}\right]\left[\begin{array}{lll}
q_{11} & q_{12} & q_{13} \\
q_{21} & q_{22} & q_{23} \\
q_{31} & q_{32} & q_{33}
\end{array}\right]\left[\begin{array}{l}
x \\
y \\
z
\end{array}\right]+L=0
$$

Reduction of the matrices to diagonal form corresponds to the transformation of coordinates, when the coordinate axes coincide with the axes of symmetry of the ellipsoid. We assume that matrix $Q(28.6)$ is reduced to the diagonal form

$$
T=D Q D^{-1}=\ldots\left[\begin{array}{ccc}
t_{1} & 0 & 0 \\
0 & t_{2} & 0 \\
0 & 0 & i t_{3}
\end{array}\right]
$$


This means that the ellipsoid equation (28.7) assumes the canonical form

$$
\begin{aligned}
& {\left[\begin{array}{lll}
x & y & z
\end{array}\right]\left[\begin{array}{lll}
t_{1} & 0 & 0 \\
0 & t_{2} & 0 \\
0 & 0 & t_{3}
\end{array}\right]\left[\begin{array}{l}
x \\
y \\
z
\end{array}\right]=} \\
& =t_{1} x^{2}+t_{2} y^{2}+t_{3} z^{2}=1 .
\end{aligned}
$$

If the Equation (28.9) is multiplied by $\mu^{2}$ (the square of the mean square error of unit weight), we will obtain the so-called mean square ellipsoid of errors

$$
t_{1}^{\prime} x^{2}+i_{2}^{\prime} y^{2}+t_{3}^{\prime} z^{2}=\mu^{2}
$$

where

$$
t_{i}^{\prime}=t_{l^{2}}
$$

In the space of an arbitrary number of measurements, Formula (28.9) assumes the form

$$
t_{1} x_{1}^{2}+t_{2} x_{2}^{2}+\ldots+t_{k} x_{h}^{2}=1
$$

and describes an ellipsoid in a multidimensional space.

Thus, the eigenvalues of the matrices of the coefficients of normal equations are the inverse of the squares of the semiaxes of a multidimensional ellipsoid. The elgenvalues of a reciprocal matrix will be equal to the welght coefficients for the adjusted values of the unknowns only in the case when the axes of the coordinate base coincide with the principal axes (the axes of symmetry) of the ellipsold (28.11). Taking the fact into account that the elgenvalues of matrix $F$ and 1 ts reciprocal matrix $Q$ are connected by the relation

$$
\frac{1}{t_{l}}=\lambda_{l}
$$


we can state that, after matrix $F$ has been reduced to diagonal form, its eigenvalues will be equal to the welghts of the adjusted values of the unknowns on the base, comprised of the eigenvectors 1120 of matrix F, i.e.,

$$
q_{i i}^{\prime}=t_{l i} \quad p_{x_{i}^{\prime}}=\lambda_{l}
$$

Non-quadratic coeficients appear in the arbitrary coordinate system in the ellipsoid equation (28.11) in exactly the same manner as for the second order surface equation, when it is located arbitrarily with respect to the coordinate axes in three-measurement space.

A number of relationships exist between the coefficients of the ellipsoid equation which are invariant with respect to the coordinate transformations. One of these invariants is the sum of the coefficients in the case of the squares of the running coordinates. For matrices, these will be diagonal elements. Their sum is called the trace of the matrix and is denoted by $\mathrm{Sp}$. Accordingly, for matrix $\mathrm{F}$ we will have

$$
S p F=f_{11}+f_{22}+\ldots+f_{k k}=\lambda_{1}+\lambda_{22}+\ldots+\lambda_{k \prime} .
$$

Let us rewrite this expression in another form with consideration of Formula (28.13)

$$
\frac{1}{k} S p F=\frac{1}{k} \sum_{i=1}^{h} \lambda_{i}=\frac{1}{k_{i}} \sum_{i}^{k} p_{x_{i}^{\prime}}
$$

Setting $\frac{1}{k} \sum_{i}^{k} p_{n i}^{\prime}:=p_{\mathrm{cp}}^{\prime}$, we obtain

$$
p_{c p}^{\prime}=\frac{1}{k} \sum_{i d j}^{k} f_{i i}
$$


From (28.16) we have the relation: the mean value of the weight of the adjusted coordinates in geodetic networks is equal to the mean value of the sum of the quadratic coefficients of the normal equations.

Formula (28.16), like the theorem on the mean ratio of weights, is the generalized characteristic of the accuracy of the network structure. However, unlike the latter, it takes into account the effect of both the number of redundant measurements, the form of the network, and the composition of the measurements from which the given network is constructed. Thus, this formula estimates the accuracy of networks of identical configuration, but with a different composition of measurements, or the accuracy of networks of different shape. Of course, Formula (28.16) may be used only for comparative analyses provided that the systems of weights in the compared networks are determined in the same manner ( $1 . e .$, based on a single error value of unit weight). The accuracy of calculating the points in the networks will then be higher, the greater the value of $\mathrm{p}_{c p}$, calculated by Formula (28.16). The generalized characteristics of accuracy, obtained according to the theorem of the mean ratio of weights or the formula for the mean weighting value, are essentially useless in an analysis of the accuracy of the position of a specific point in an adjusted network. It is a very complex problem to obtain a priori estimates from the elements of the reciprocal matrix and analysis of them. However, taking the fact into account that a priori analysis is in one way or another approximate, the requirements on the accuracy of calculating the elements of the reciprocal matrix and the requirement to use approximate methods of its construction naturally decrease. 
Henceforth, we shall use those methods of a priori accuracy analysis, from those described in this section, which reduce more conveniently to a solution of the given specific problem.

\section{Errors in Space Triangulation Elements}

The entire range of the elementary figures of space triangulation, previously considered in Chapter 4, Sections 17 and 18, with respect to the a priori analysis of the accuracy of the position of observation points calculated by these figures, may be essentially reduced to three basic elementary figures:

- intersection of two directions (three-dimensional angular intersection),

- intersection of the direction and the plane,

- intersection of three planes (intersection of planes).

In this case, the third type of figure - intersection of planes - may in turn be reduced to a second type, because it may be assumed to be the intersection of a single plane with the direction of the line of intersection of two other planes.

Obviously, the main elements of these figures are the direction and the plane.

Because of the characteristics of space triangulation construction, the relationships between the measured values and their functions are much more complex than those in ordinary triangulation networks, since the position of points in space triangulation is often calculated with the aid of intermediate elements, which are very complex functions of the measured values. Therefore, consideration of the problems of a priori 
accuracy analysis naturally begins with determination of the relationships between the errors in directly measured values and those in calculating the geometric elements of space triangulation.

In order to derive the formulas for a priori analysis of the accuracy of the position of points - the apexes of elementary figures - the errors in the elements of space triangulation may be represented in the form of linear shifts from the "true" position of the points. Accordingly, we are interested in the linear deviation of the plane passing through two points from one of them (calculated) - due to the errors in measuring the values which determine the position of the plane - rather than the total error of the position of the plane in space, which is naturally characterized by the shift of its normal vector $\bar{N}$.

Similarly, we are interested in the linear displacement of the direction in the plane, perpendicular to it and passing through a specific point, rather than in the angle at which the directional error may be represented, i.e., the angular value of the deviation /122 of direction from its "true" position.

We shall use the following notation when considering the expressions for the errors in the elements of space triangulation:

\footnotetext{
$M$ - the error in the position of the apex of the figure, $\mathrm{m}_{\mathrm{H}}$ - the directional error (linear displacement),

$\mathrm{m}_{\mathrm{p}}$ - the error in the position of the plane,

$m_{\delta}$ - the error of the topocentric declination of the AES,

$\mathrm{m}_{\gamma}$ - the error in the hour angle of the AES,

$m_{B}$ - the error in the direction from the point to the AES position,
} 
$m_{\rho}$ - the error in the distance from the point to the AES position,

$\mathrm{m}_{\Delta \rho}$ - the error in the difference of distances from the observation point to two AES positions,

$\mathrm{m}_{\Phi}$ - the error in "declination" of the chord,

$\mathrm{m}_{\Lambda}$ - the error in the "hour angle" of the chord,

$\mathrm{m}_{\mathrm{B}}$ - the error in the direction of the chord,

$\mathrm{m}_{\mathrm{D}}$ - the error in the base of the chord,

$m_{d}$ - the error in the base of the celestial chord (the distance between the AES positions),

$\mathbb{M}_{k}$ - the error in the AES position,

$M_{1}$ - the error in the position of the starting point,

$\mathrm{M}_{\mathrm{f}}$ - the error in the position of the observation point to be calculated,

$\tau$ - the distance of the observation point from the plane normal to the celestial chord and drawn through its center,

$\lambda$ - the angle between the planes,

$\varphi$ - the angle between the directions,

$\psi$ - the angle between the direction and the plane.

Errors in the Observation Point-Satellite Vector

In photographic observations, the direction from the observation point to the AES position is calculated by the two measured values $\gamma$ and $\delta$. The accuracy of calculating the direction will be characterized by the value of the displacement vector, which 
is located in the plane perpendicular to the given direction and which passes through the calculated point, or by the angular value

$$
\Delta \beta=\frac{\left|\overline{\rho_{\rho}}\right|}{|\bar{\rho}|}
$$

where $\Delta \beta$ is the angle between the true and actually obtained directional position; $\bar{d} \bar{\rho}$ is the displacement vector; and $\bar{\rho}$ is the vector coincldent with the measured direction.

For the mean square error in the direction $m_{\beta}$, we will have

$$
m_{\beta}=\frac{!_{H}}{|\underline{\rho}|}
$$

where $m_{H}$ is the mean square value modulo the displacement vector.

Then, differentiating the expression for the direction co- $/ 123$ sines (16.1) by the variables $\gamma, \delta, x, y, z$ and converting to the mean square errors, we obtain

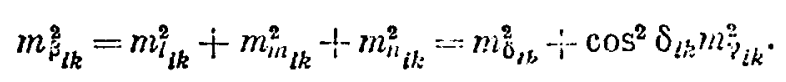

The mean square value of the linear directional displacement, on the basis of (29.2), will be

$$
m_{\mathrm{a}}=\rho_{i k} m_{i_{i k}}
$$

In photographic observations, cases are possible when the time of photographing is unknown and because of this, only the declination of the AES position, rather than the two values which characterize the direction from the point to the AES position, is obtained from the astrometric processing of the photograph. 
The declination defines the surface of a cone with an axis perpendicular to the equatorial plane, passing through the point and AES position. Because of the error in the declination, the surface of the cone deviates from the AES position. The conical surface near the AES position may be interpreted as the plane, tanget to the generatrix of the cone. In this case

$$
m_{p}=\rho_{i k} m_{i k},
$$

but, taking (29.3) into account and assuming $\mathrm{m}_{\delta}^{2}=\mathrm{m}_{\delta}^{2} \cos ^{2} \delta$,

$$
\mathrm{m}_{\mathrm{p}}=\rho_{i k} \frac{m_{\beta_{i k}}}{\sqrt{2}}
$$

When the angular and linear measurements in space triangulation are combined, the spherical surface, defined by the measured distance from the observation point to the AES position, may be approximated in a priori accuracy analysis by the plane tangent to the sphere at the end of the measured radius vector and, consequently, normal to the latter.

In this case the error in the distance will determine the position error of the plane

$$
\mathrm{m}_{\mathrm{p}}=m_{\rho_{1 k}} \text {. }
$$

If the photographic observations are combined with Doppler observations, cases are possible when the direction to the AES position and the difference in distances to this and another AES position are calculated from the point. For a priori analysis of the accuracy of triangulation structures, each measured value of 
the difference in distances $(\Delta \rho)$ may be interpreted geometrically as the surface of a double-surface hyperboloid of rotation, on which lies the observation point and whose foc 1 are the two AES positions, the difference in distances to which is measured (as the difference in focal radi1). In this case, the modulus of the observation point-AES position vector may be defined as the length of the focal radius of the hyperbolold.

Since we are interested in the elements of the internal geometry of the space triangulation figure independently of 1 ts position with respect to the geocentric coordinate system, we use the canonical equation of the double-surface hyperbolold of rotation

$$
\frac{x^{2}+y^{2}}{a^{2}}-\frac{x^{2}}{c^{2}}=\sin 1
$$

on the basis of which and of our assumed notations (Figure 42), the length of the focal radius is

$$
\rho_{i k_{1}, i k_{2}}=\frac{d_{k_{1} k_{2} l_{i}}}{\Delta \rho_{i}} \pm \frac{\Delta \rho_{i}}{2}
$$

where the sign in front of the second term is defined by the near or far focus of the hyperboloid (the AES position) with respect to the point.

Differentiating this expression with respect to $\Delta \rho$ - the measured value - and changing the mean square errors, we obtain the formula for estimating the accuracy of the length of the focal radius or the distance from the point to the AES position

$$
m_{r_{l k}}^{2}=\left(\frac{d l_{l}}{\Delta \rho^{2}} \pm \frac{1}{2}\right)^{2} m_{\Delta ? \cdot}^{2}
$$




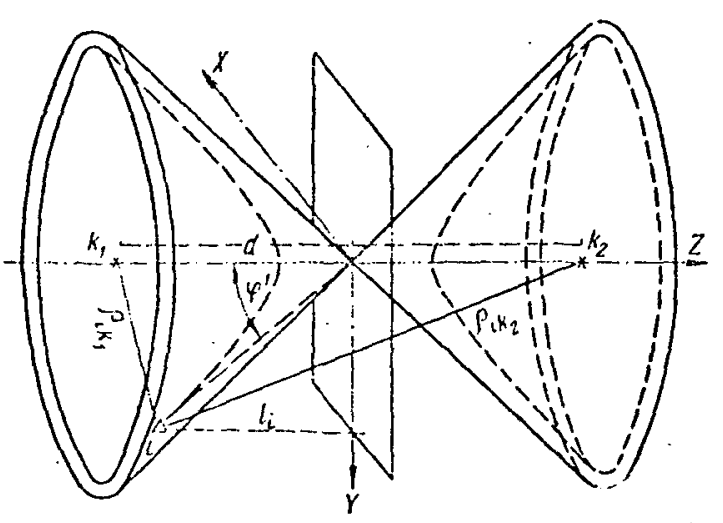

Figure 42.

Simple calculations show that disregard of the second term in parentheses distortes the result in the worst case by no more than 25\%. Therefore, taking into account (29.7), we can write Formula (29.10) in the form

$$
\mathrm{m}_{\mathrm{p}}^{2}=m_{\rho_{l k}}^{2}=\frac{d^{2} l^{2}}{\Delta \rho^{t}} m_{\Delta \rho}^{2}
$$

The Position Error of the Plane

Since we will assume that the synchronization plane passes through one of the points and contains both directions, we will artificially transfer the direction from the second point parallel to itself by the value of the shortest distance between the cross directions. Accordingly, the synchronization plane is also shifted with respect to the second point by the value of this distance $\mathrm{m}_{\mathrm{p}}$. This displacement is the normed value of the free term of the conditional equation of the synchronization plane and may be obtained in the process of a posteriori determination of accuracy.

The shortest distance between the intersecting straight Iines may be represented as the difference in the projections of the displacement of the directions due to their errors on the normal to the plane

$$
\Delta_{p}=\left|\bar{\Delta}_{\mathrm{H}_{1}}\right| \cos \alpha_{1}-\left|\bar{\Delta}_{\mathrm{H}_{2}}\right| \cos \alpha_{2}
$$

where $\alpha$ is the angle between the error vector of the direction $\bar{\Delta}_{H}$ and the normal to the plane of intersection (the synchronization plane). 
Assuming that $\alpha$ is a random value with unfform distribution, we obtain for the mathematical expectation $\cos ^{2} \alpha$

$$
M\left(\cos ^{2} \alpha\right)==\int_{0}^{2 \pi} \cos ^{2} \alpha \frac{d \alpha}{2 \pi}=\frac{1}{2}
$$

Converting from (29.12) to the mean square errors, we obtain

$$
\mathrm{m}_{\mathrm{p}}^{2}=\frac{m_{\mu_{1}}^{2}+m_{H_{2}}^{2}}{2}
$$

Taking into account (29.4)

$$
\mathrm{m}_{\mathrm{p}}^{2}=\frac{\rho_{1 k}^{2} m_{\hat{\beta}_{1} 1 k}^{2}+\rho_{2 k}^{2} m_{\mathrm{P}_{2 k}}^{2}}{2} .
$$

With uniformly precise measurements

$$
\mathrm{m}_{\mathrm{p}}^{2}=\frac{m_{p}^{2}}{2} \sum_{i=1}^{2} \rho_{i k}^{2}
$$

The ratio of the values $\rho_{1}$ and $\rho_{2}$ in photographic observations does not exceed 2 in the case when an AES at an altitude of $\mathrm{H} \cong 1000 \mathrm{~km}$ is observed from one point at its zenith, and from another point at the minimum elevation above the horizon $\left(z=70^{\circ}\right)$. In all remaining cases, as well as when the altitude of the AES increases, this ratio will tend toward unity.

Substitution of the values $\rho_{1 k}$ in Expression (29.16) for the average distance from the points to the AES position, $\rho_{c p_{k}}$, even with the indicated maximum difference of values, $\rho_{1 k}$ changes the result by 10\%. In most cases, this substitution hardly changes the result. Therefore, finally

$$
\mathrm{m}_{\mathrm{p}}=\rho_{\mathrm{cr}_{k}} m_{3}
$$


As when determining the error in the position of the synchro- 1126 nization plane, we are interested in the deviation of the hyperboloid surface from the point through which the latter should pass, for measured differences in distances. This deviation is caused by the error in calculating the difference in distances, corresponding to this surface. To solve this problem, the surface of the hyperboloid near the observation point may be approximated with a sufficient degree of approximation by the surface of an asymptotic cone with a vertex in the center of the celestial chord.

The canonical equation of the hyperboloid makes it possible to derive an expression for angle $\varphi^{\prime}$ between the real axis of the hyperbolold (the direction of the celestial chord) and the generatrix of the asymptotic cone (see Figure 42 ).

$$
\varphi^{\prime}=\operatorname{arctg} \sqrt{\frac{d^{2}}{\Delta \rho^{2}}-1}
$$

Linear displacement of the surface of the cone $\Delta_{p}$ from position of the point, separated from the vertex of the cone by distance $\rho_{c p}$, due to a variation of the value of angle $\varphi^{\prime}$, will be

$$
\Delta_{\mathrm{p}}=\rho_{\mathrm{cp}} d \varphi^{\prime}
$$

Having differentiated Expression (29.18) with respect to $\Delta \rho$, having substituted the result obtained into (29.19) and converting to the mean square errors, we obtain an approximate formula for the error in the position of the hyperbolold, which causes an error in the measurement of the distance differences. This error near the point may be considered the error in the position of the plane, tangent to the generatrix of the asymptotic cone 


$$
\mathrm{m}_{\mathrm{p}}^{2}=\frac{\rho_{\mathrm{cp}}^{2}}{d^{2}-\Delta p^{2}} m_{\Delta \rho}^{2}
$$

\section{Errors in the Chord Elements}

During construction and analysis of the accuracy of space triangulation, the figures and networks, formed directly by the chords between the observation points, may be considered. However, the chords and elements which characterize them are complex functions of the measurement results and are obtained in turn from the elementary figures, containing - besides the two points the ends of the chord, the required AES positions.

As mentioned in section 16, the direction of the chord is calculated as a result of the intersection of two planes, each of which is formed by photographic observations of a single AES position from two points - the ends of the chord. Thus, the elementary figure for calculating the direction of the chord contains two pairs of directions from the points to the AES (four pairs of measured values) (F1gure 43 ).

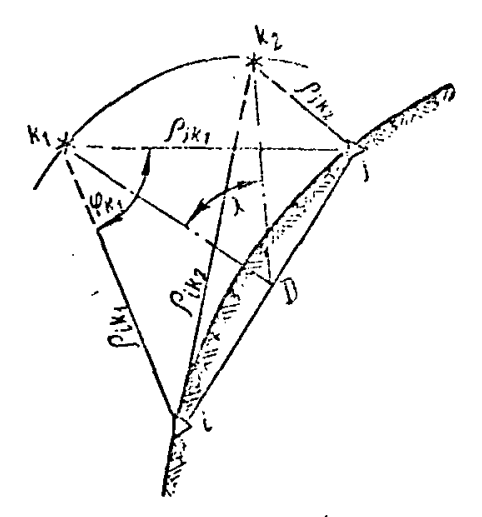

Figure 43.

The error in the direction of the chord will be calculated in a manner similar to the error in the direction from the point to the AES position (29.3) by the ratio of the displacement of the chord end $m_{H}$ to its length $D$ :

$$
\left.m_{\mathcal{B}}^{2}==m_{\Phi}^{2}+m_{\Lambda}^{2} \cos \Phi\right) \cdots \frac{m_{\mathrm{\Lambda}}^{2}}{D^{2}}
$$

Displacement of the chord end in a $\underline{127}$ plane, perpendicular to the chord direction $\left(m_{H}\right)$, is caused by the errors in the position of the synchronization planes, located in the same plane, intersection of which 
determines the direction of the chord. Consequently, to find the value of chord displacement, the well-known formula for the error in the position of the apex of angular intersection in a plane may be used

$$
m_{11}^{2}=\frac{m_{\mathrm{p}}^{2} \mathrm{i}^{+m^{2}} \mathrm{p}_{2}}{\sin ^{2} \gamma^{2}}
$$

with consideration of (29.15)

$$
m_{11}^{2}=\frac{\sum_{i=1}^{2} \sum_{k=1}^{2} \dot{\rho}_{i k}^{2} m_{\psi_{i k}}^{2}}{2 \sin ^{2} \%}
$$

From Expression (29.23), we obtain the formula for the error in the direction of the chord

$$
m_{L}^{2}=\frac{\sum_{i=1}^{2} \sum_{k=1}^{2} \rho_{i k}^{2} m_{i, i k}^{2}}{2 D^{2} \sin ^{2} j}
$$

Displacement of the direction of the chord due to its error in uniformly precise measurements will be

$$
m_{\mathrm{k}}^{2}=\frac{\sum_{i=1}^{2} \sum_{k=1}^{2} \rho_{i k}^{2}}{2 \sin ^{2} \hat{i}} m_{i}^{2}
$$

and taking into account (29.17)

$$
m_{\mathrm{H}}^{2}=\frac{\sum_{k=1}^{2} \rho_{\mathrm{c}_{\mathrm{p} k}}^{2}}{\sin ^{2} \lambda} m_{j}^{2},
$$

and the error in the direction of the chord

$$
m_{13}^{2}=\frac{\sum_{k=1}^{2} \rho_{\mathbf{c p}_{k}}^{2}}{D^{2} \sin ^{2} \lambda_{j}} m_{j}^{2} .
$$




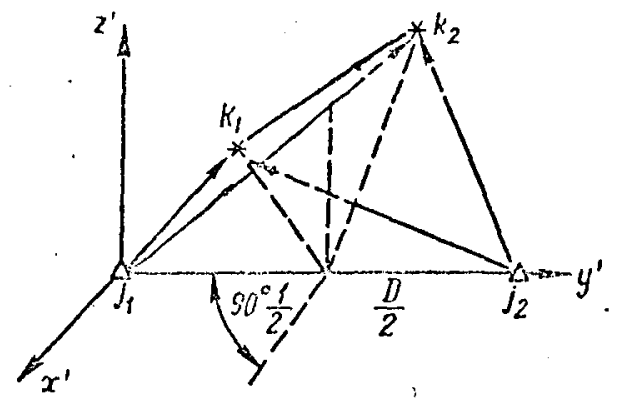

Figure 44.

In the latter case $\rho_{c p}$ may be expressed by the angle of intersection $\varphi k$ for an AES (see Figure 44)

$$
\rho_{\mathrm{cp}}=\frac{D}{2 \sin \frac{\varphi}{2}} .
$$

From (29.27), taking into account (29.28), we may obtain

$$
m_{i 3}^{2}=\frac{m_{3}^{3}}{2 \sin 2 \lambda \sin ^{2}-\frac{4 c_{2}-2}{2}} \text {. }
$$

This same expression may be obtained by inversion of the matrix of normal equations, which are formed in the figure for calculating the chord direction.

Two synchronization planes, formed by observations of two satellite positions $k_{1}$ and $k_{2}$ from two points $j_{1}$ and $j_{2}$, are shown in Figure 44.

Let us select a system of three-dimensional rectangular Descartes coordinates such that the OY-axis is parallel to the chord $j_{2} j_{2}$, and the ZOY plane equally divides the two-sided angle $\lambda$, formed by the synchronization planes.

Let us write the equations of the two synchronization planes $(k=1,2)$

$$
\begin{gathered}
F_{k}=A_{k} \cos \Lambda \cos \Phi+B_{k} \sin \Lambda \cos \Phi+1 \\
+C_{k} \sin \Phi=0
\end{gathered}
$$


Taking the fact into account that in the assumed coordinate system, the angles which characterize the direction of the chord, are $\Lambda=90^{\circ}$ and $\Phi=0$, we obtain from (29.30) the error equations:

$$
-A_{k} d \Lambda+C_{k} d \Phi+W_{k}=\varepsilon_{k}, \quad \text { weight } \mathrm{p}_{\mathrm{k}}
$$

Each of the two equations (29.31) is a function of four measured values; therefore, their weights are calculated by the formula

$$
p_{k}=1:\left\{\frac{\left(\frac{\partial F_{k}}{\partial \gamma_{j_{1} k}}\right)^{2}}{P_{\gamma_{j_{1} k}}}+\frac{\left(-\frac{\partial F_{k}}{\partial \delta_{j_{1} k}}\right)^{2}}{p_{\delta_{j_{1} k}}}+\frac{\left(\frac{\partial F_{k}}{\partial \gamma_{j_{2} k}}\right)^{2}}{p_{\gamma_{j_{2} k}}}+\frac{\left(\frac{\partial F_{k}}{\partial \delta_{j_{2} k}}\right)^{2}}{p_{\delta_{j_{9} h}}}\right\} .
$$

Let us set $\gamma_{J_{1} 1}=\gamma$ and $\delta_{J_{1} 1}=\delta$; then

$$
\gamma_{j_{1} 1}=360^{\circ}-\gamma ; \quad \gamma_{j_{1} 2}=180^{\circ}-\gamma ; \quad \gamma_{j_{2} 2}=180^{\circ}+\gamma 11 \quad \delta_{j_{1} 2}=\delta_{j_{2} 1}=\delta_{j_{2} 2}=\delta \text {. }
$$

According to Expressions (29.31) and (29.32) and taking into account the values of the angles, the matrices of coefficients $K$ and of weights $P$ of the correction equations assume the form

$$
\begin{gathered}
K=\left[\begin{array}{cc}
-2 \operatorname{tg} \delta \sin \gamma & \sin 2 \gamma \\
-2 \operatorname{tg} \delta \sin \gamma & -\sin 2 \gamma
\end{array}\right], \\
P=\left[\begin{array}{cc}
\frac{\cos ^{4} \delta}{2\left(\sin ^{2} \gamma \sin ^{2} \delta-1 \cdot \cos ^{2} \gamma\right)} & 0 \\
0 & \frac{\cos \delta}{2\left(\sin ^{2} \gamma \sin ^{2} \delta+\cos ^{2} \gamma\right)}
\end{array}\right] .
\end{gathered}
$$

Let us compile the matrix of coefficients of normal equations

$$
N=K^{T} P_{K}
$$


and, having transformed it, we obtain the matrix of the weight coefficienț :

$$
Q=\left[\begin{array}{cc}
\frac{\sin ^{2} \gamma \sin ^{2} \delta+\cos ^{2} \gamma}{\sin ^{2} \gamma \sin ^{2} 2 \delta} & 0 \\
0 & \frac{\sin ^{2} \gamma \sin ^{2} \delta+\cos ^{2} \gamma}{\sin ^{2} 2 \gamma \cos ^{1} \delta}
\end{array}\right] .
$$

Taking into account the relations $\sin \gamma=\frac{D}{2 \varphi \cos \delta} ; \cos \gamma=\frac{D}{2 \rho \cos \delta} \operatorname{ctg} \frac{\varphi}{2} \sin \frac{\lambda}{2} \quad$ and $\bar{d}^{-1} \sin \delta=\cos \frac{\varphi}{2} \cos \frac{\lambda}{2}$

we transform the matrix (29.35)

$$
Q=-\left[\begin{array}{cc}
\frac{1}{4 \cos ^{2}-\frac{\lambda}{2} \sin ^{2}-\frac{\pi}{2}} & 0 \\
0 & 4 \sin ^{2} \frac{1}{2} \sin ^{2} \frac{Q}{2}
\end{array}\right] .
$$

Directly from (29.36), introducing the error of unit weight $\mu$, we obtain expressions for the errors in calculating angles $\Lambda \cos \Phi$ and $\Phi$, as well as those for the total error in the direction of the chord $m_{B}$

$$
\begin{gathered}
m_{\Lambda} \cos ()=\frac{\mu}{2 \cos \frac{\lambda}{2} \sin \frac{\varphi}{2}}, \\
m_{\mathfrak{d}}=\frac{\mu}{2 \sin \frac{\lambda}{2} \sin \frac{\varphi}{2}}, \\
m_{B}=\frac{\mu}{\sin \lambda \sin \frac{\varphi}{2}} .
\end{gathered}
$$

Knowing that $\mu=m_{B} / \sqrt{2}$, we obtain the same expression (29.29).

When photographic observations are combined with measured distances, the elementary figure for calculation of the chord base (assuming that the chord direction has already been calculated) should contain the directions from the observation points 
to one AES position and the measured distance from one of the observation points to this same AES position (see Figure 16, where angles $\beta$ are replaced by $\varphi$ ).

Differentiating the expression for chord base

$$
D=\cdots \rho_{i k} \frac{\sin \left(\varphi_{i}+-\varphi_{i}\right)}{\sin \varphi_{j}}
$$

with respect to $\rho_{i k}$, we obtain

$$
\frac{d D}{D}=-\frac{d \rho_{l k}}{\rho_{i k}}+\operatorname{ctg} \varphi_{k l} d \varphi_{i}-\frac{\rho_{j k}}{\rho_{i k} \sin \varphi k} d \Upsilon_{j}
$$

When photographic observations are combined with measurement of the differences in distances from an observation point to two AES positions, the elementary figure for calculating the chord base should contain, besides the directions from two observation points - the ends of the chord - to two AES positions, and one difference in distances, measured from one of the observation points, to these AES positions (see Figure 16).

Differentiating the expression for chord base

$$
D=\Delta \rho_{i} \frac{\sin \left(\varphi_{i 2}+\varphi_{i 1}\right) \sin \left(\varphi_{i 2}+\varphi_{i j}\right)}{\sin \varphi_{i 1} \sin \left(\varphi_{i 2}+\varphi_{j i}\right)-\sin \varphi_{i 2} \sin \left(\varphi_{i 1}+\varphi_{i j}\right)},
$$

we will have

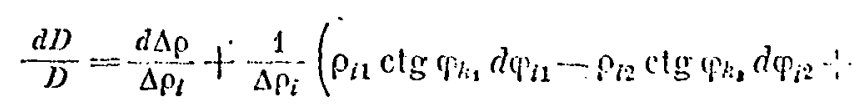

$$
\begin{aligned}
& \left.+\frac{\rho_{j_{1}}}{\sin \varphi_{h_{1}}} d \varphi_{j_{1}}-\frac{\rho_{j_{2}}}{\sin \varphi_{h_{2}}} d \varphi_{j_{2}}\right) \text {. }
\end{aligned}
$$


A value appeared in Formulas (29.39) and (29.41), which we $/ 130$ did not consider previously, the error in the angle between the directions toward the AES - $m_{\varphi}$. Since the directions toward the AES position are crossed due to errors, the angle between them is formed only after they become coplanar, 1.e., one of them will be displaced parallel to itself by the value of the shortest distance between the directions.

Assuming that the error in the angle will be equal to the difference in the projections of direction errors on the plane

$$
d \varphi=\left(\frac{\left|\ddot{\Delta}_{H}\right| \sin \alpha}{\rho}\right)_{1}-\left(\frac{\left|\bar{\Delta}_{H}\right| \sin \alpha}{\rho}\right)_{2}
$$

and converting to mean square errors, taking into account (29.13), we obtain the expression for the error in the angle between directions

$$
m_{?}^{2}=\frac{m_{\beta_{1}}^{2} \div m_{\beta_{2}}^{2}}{2}
$$

or with equally precise measurements

$$
m_{\stackrel{\leftrightarrow}{2}}^{2}=m_{i}^{2} .
$$

The result should not be unexpected, since the error in angle $\mathrm{m}_{\varphi}$ will be affected by only those components of the errors in the topocentric coordinates $\gamma$ and $\delta$, which are located in the plane of the angle itself.

Formula (29.43) is general. An expression may be easily obtained from it for the error in the angle formed by the direction toward the AES of a chord and two chords. 
Converting to the mean square errors in (29.39) and using (29.44) for $m_{\varphi_{k}}$ and $m_{\varphi_{1}}$, we obtain for the error in the chord base, which depends on the error in the angular measurements and the measurements of distances

$$
\begin{gathered}
\mathrm{m}_{\mathrm{p}}^{2}=m_{L}^{2}=\frac{D^{2} m_{P}^{2}}{\rho_{i k}^{2}}+\frac{D^{2}}{2 \sin ^{2} \varphi_{k} \sigma_{i k}^{2}} \times \\
\times\left[\left(\cos ^{2} \varphi_{k}+\frac{\rho_{j k}^{2}}{\rho_{i k}^{2}}\right) m_{\mathrm{p}}^{2}+\left(\cos \varphi_{k}-\frac{\rho_{j k}}{\rho_{i k}}\right)^{2} m_{B}^{2}\right] .
\end{gathered}
$$

Converting in a similar manner to the mean square errors in (29.41), and taking into account Expressions (29.44) for the error in angles $\varphi$, we obtain the expression for the error in the chord base and, consequently, the error in the position of the plane normal to the chord, as a function of the errors in the angular measurements and measurements of the difference in distances

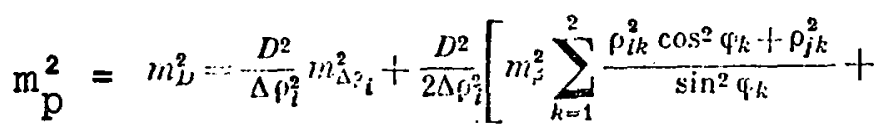

$$
\begin{aligned}
& \left.+m_{j}^{2} \sum_{k=1}^{2} \frac{\left(\rho_{i k} \cos \varphi_{k}-\rho_{j k}\right)^{2}}{\sin ^{2} \varphi_{k}}\right] \text {. }
\end{aligned}
$$

30. Estimation of the Accuracy of Elementary Figures

The Error in the Vertex of Three-Dimensional Angular Intersection

The directions from two points to the vertex of threedimensional angular intersection, as already mentioned previously, do not intersect in the general case due to errors and are crossed. The distance of the trace of each direction in the plane, normal to it and passing through the vertex, from the intersection plane will be equal to $\left|\overline{\Delta_{H}}\right| \sin \alpha$, where $\overline{\Delta_{H}}$ is the 


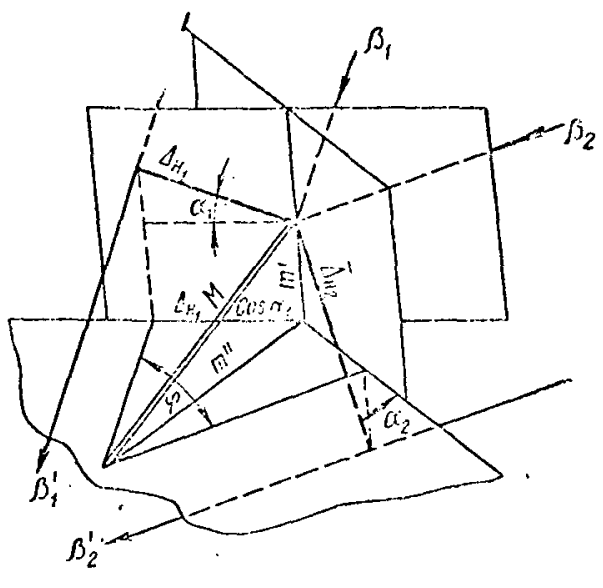

Figure 45. shift in direction from the vertex and $\alpha$ is the angle formed by vector $\Delta_{\mathrm{H}}$ with the intersection plane (Figure 45).

Intersection of the directions will be possible after their reduction in the intersection plane, separated from the point to be calculated by the value obtained as the mean weight of the displacements of both directions.

The mean square value of this value will be

$$
m^{\prime 2}=\frac{1}{2}\left(\frac{m_{\mathrm{H}_{1}}^{2} m_{\mathrm{H}_{2}}^{2}}{m_{\mathrm{H}_{1}}^{2}+m_{\mathrm{H}_{2}}^{2}}\right)
$$

The projections of the direction displacements on the intersection plane will be equal to $\Delta_{\mathrm{H}_{1}} \cos \alpha_{1}$ and $\Delta_{\mathrm{H}_{2}} \cos \alpha_{2}$, and the projections of the directions will intersect at the intersection angle $\varphi$. The point of intersections of the direction projections will then be separated from the projection of the true position of the vertex on this plane by a value which in essence is the error in the angular intersection in the plane. Therefore, we can write

$$
m^{2}=\frac{1}{2}\left(\frac{m_{\mathrm{H}_{1}}^{2}+m_{\mathrm{H}_{2}}^{2}}{\sin ^{2} \varphi}\right)
$$

The error in the position of the intersection vertex will be calculated by the expression

$$
M^{2}:=m^{\prime 2}+m^{\prime 2}=\frac{1}{2}\left(\frac{m_{\mathrm{H}_{2}}^{2} m_{\mathrm{H}_{2}}^{2}}{m_{\mathrm{H}_{1}}^{2}+m_{\mathrm{H}_{2}}^{2}}+\frac{m_{\mathrm{B}_{1}}^{2}+m_{\mathrm{H}_{2}}^{2}}{\sin ^{2} \varphi_{4}}\right),
$$


and with equally precise measurements

$$
M^{2} \therefore m_{11}^{2}\left(\frac{1}{4}+\frac{1}{\sin ^{2} 4}\right)
$$

Taking into account that disregard of the value $1 / 4$ in the parentheses of Expression (30.4) will alter the value of the error (reduce it) by a total of 10\% in the maximum case (at $\varphi=$ $\pi / 2$ ), for simplicity we may assume

$$
M^{2}=: \frac{m_{u}^{2}}{\sin ^{2} \varphi}
$$

The formula for the error in the position of the vertex of 1132 three-dimensional angular intersection (Expressions $30.3-30.5$ ) is applicable when calculating the AES position by the directions toward it from two observation points, or calculating the position of the point by the directions from it toward two known AES positions. In this case, $\mathrm{m}_{\mathrm{H}}$ is calculated by Formula (29.4) and with equally precise measurements.

$$
M_{j}^{2}=\frac{m_{j}^{2}}{2}\left(\frac{\rho_{j h_{1}}^{2} \rho_{j k_{2}}^{2}}{\rho_{j h_{1}}^{2}+\rho_{j k_{2}}^{2}}+\frac{\rho_{j k_{1}}^{2}+\rho_{j k_{2}}^{2}}{\sin ^{2} \phi}\right)
$$

This expression may also be obtained directly by inversion of the matrix of normal equations, formed from measurements of the given figure.

Let angles $\gamma$ and $\delta$, which characterize the topocentric directions toward the satellite position $k$, be measured from observation points $j_{1}$ and $j_{2}$ (Figure 46). Assuming that $p_{\gamma} \cos \delta=$ $p_{\delta}=1$, we write the equations for the errors in this construction

$$
\begin{aligned}
& {\left[v_{\gamma_{1}} \cos \delta_{1} ; v_{\delta_{1}} ; v_{\gamma_{2} \cos \delta_{2}} ;\right.} \\
& \left.v_{\delta_{2}}\right]^{T}=K\left[\begin{array}{lll}
d x_{k} ; & d y_{k} ; & d z_{k}
\end{array}\right]+l
\end{aligned}
$$




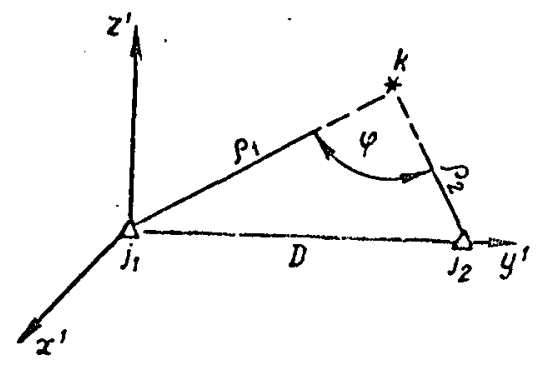

Figure 46. (subscript 1 corresponds to the observations from point $j_{1}$, and subscript 2 - from point $j_{2}$ ).

Let us assume that the chord $j_{1} j_{2}$ is parallel to the coordinate Iine oy', and the synchronization plane $j_{1} k_{j}$ is parallel to the coordinate plane z'oy'.

In the selected coordinate system, the matrix of coefficients of the equations for the errors in the measured values assumes the form

$$
K=\left[\begin{array}{ccc}
\frac{1}{\rho_{1}} & 0 & 0 \\
0 & -\frac{\sin \delta_{1}}{\rho_{1}} & \frac{\cos \delta_{1}}{\rho_{1}} \\
\frac{1}{\rho_{2}} & 0 & 0 \\
0 & \frac{\sin \delta_{2}}{\rho_{2}} & \frac{\cos \delta_{2}}{\rho_{2}}
\end{array}\right]
$$

Matrix (30.8) corresponds to the matrix of normal equations

$$
N=\left[\begin{array}{ccc}
\left(\frac{1}{\rho_{1}^{2}}+\frac{1}{\rho_{2}^{2}}\right) & 0 & 0 \\
0 & \left(\frac{\sin ^{2} \delta_{1}}{\rho_{1}^{2}}+\frac{\sin ^{2} \delta_{2}}{\rho_{2}^{2}}\right) & \left(\frac{\sin \delta_{2} \cos \delta_{2}}{\rho_{2}^{2}}-\frac{\sin \delta_{1} \cos \delta_{1}}{\rho_{1}^{2}}\right) \\
0 & \left(\frac{\sin \delta_{2} \cos \delta_{2}}{\rho_{3}^{2}}-\frac{\sin \delta_{1} \cos \delta_{1}}{\rho_{1}^{2}}\right) & \left(\frac{\cos ^{2} \delta_{1}}{\rho_{1}^{2}}+\frac{\cos ^{2} \delta_{2}}{\rho_{2}^{2}}\right)
\end{array}\right],
$$

where $\rho_{1}$ and $\rho_{2}$ are the distances from the observation points $j_{1}$ and $f_{2}$, respectively, to the satellite position $k$.

By inverting matrix $N$, we obtain the matrix of the welght coefficients 


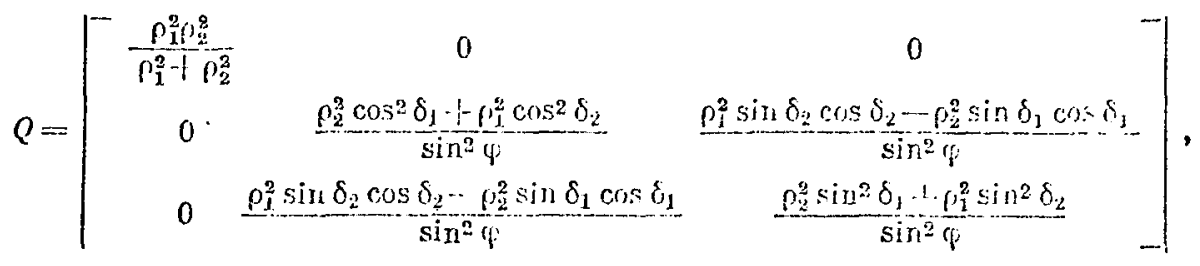

where $\varphi$ is the angle of intersection of the directions for the satellite.

Let us introduce the error in unit weight $\mu=\mathrm{m}_{\gamma} \cos \delta=$ $m_{\delta}=m_{\beta} \sqrt{2}$, and from $(30.10)$ we obtain the expression for the square of the error in the satellite position:

$$
M_{k}^{2}=\mu^{2}\left(\frac{\rho_{1}^{2} \rho_{3}^{2}}{\rho_{1}^{2}-1-\rho_{2}^{2}}+\frac{\rho_{1}^{2}+\rho_{2}^{2}}{\sin ^{2} \phi}\right) \text {, i.e., Expression }(30.6)
$$

Expression (30.6) is applicable for an analysis of the accuracy of the position of the point, calculated at the intersection of the chord directions, which connect it to two other points. In this case, the errors in the directions $\mathrm{m}_{\beta}$ in Formula (30.6) are replaced by the errors in the directions of chords $m_{B}$, and the distances to the AES positions $\rho_{j k}-$ by the chord lengths $D_{i j}$.

The Error in the Position of the Point where the Direction Intersects the Plane

Geometric interpretation of the effect of errors in the direction and plane on the error in the position of the point of their intersection is presented in Figure 47. 


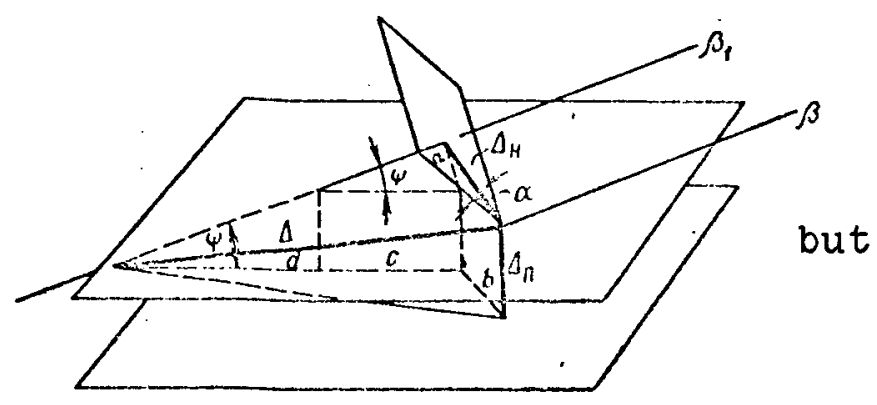

Figure 47.

From Figure 47 we have

$$
\Delta^{2}=\Delta_{\mathrm{n}}^{2}+b^{2}+(c+d)^{2}
$$

$$
\begin{aligned}
& \mathrm{b}=\Delta_{\mathrm{H}} \cos \alpha, \\
& \mathrm{c}=\alpha \operatorname{cosec} \psi, \\
& \mathrm{a}=\Delta_{\mathrm{H}} \sin \alpha, \\
& \mathrm{d}=\Delta_{\mathrm{p}} \operatorname{ctg} \psi,
\end{aligned}
$$

where $\psi$ is the angle of intersection of the direction with the plane.

Having substituted the values $a, b, c$, and $d$ from (30.12) into (30.11) and converting to the mean square errors, taking into account Formula (29.11), we obtain the expression of the mean square error at the point of intersection of the direction with the plane

$$
M^{2}=\frac{m_{\mathrm{I}}^{2}}{2}+\frac{\frac{m_{\mathrm{ll}}^{2}}{2}+m_{\mathrm{II}}^{2}}{\sin ^{2} \psi}
$$

The formula for the error in the point of intersection of the direction with the plane may be used for a priori analysis of accuracy of a rather large number of elementary figures.

Formula (30.13) may be used in photographic observations to estimate the accuracy of the position of the point, calculated by intersection of the chord direction, connecting it to one starting point, with the synchronization plane passing through the calculated and second starting point. 
In this case $\mathrm{m}_{\mathrm{H}}$ is calculated by Expression (29.26), and $m_{p}$ - by Expression (29.16) and

$$
M_{j}^{2}=\frac{1}{2}\left(D_{i_{i j}}^{2} / m_{B_{i, j}}^{2}+\frac{D_{i_{i} j}^{2} m_{B_{i_{1} j}^{2}}^{2}+\left(\rho_{i_{k} k}^{2}+\rho_{j k}^{2}\right) m_{B}^{2}}{\sin ^{2} \psi}\right) .
$$

The expression for the error in the point of intersection of the direction of the chord with the synchronization plane may be obtained with the aid of the inversion matrix.

Let two AES positions be observed from points $i_{2}$ and $j$ (Figure 48), as a result of which the direction of the chord $1_{2} j$ (angles $\Lambda$ and $\Phi$ ) is calculated by intersection of the two synchronization planes. Moreover, a third satellite position $k_{3}$ was observed from points $i_{2}$ and $j$. The position of point $j$ ( $i_{1}$ and $i_{2}$ are the starting points) is calculated at the intersection of chord $i_{2} j$ with the synchronization plane $i_{1} k_{3} j$.

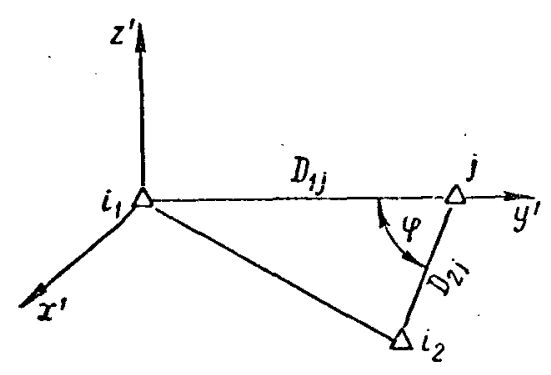

Figure 48.
Let us select a coordinate system such that chord $i_{2} j$ is parallel to the coordinate plane $z^{\prime} o x '$, chord $i_{1} j$ is parallel to coordinate line oy', and the synchronization plane $i_{1} k_{3} j$ is parallel to the coordinate plane $x^{\prime} \circ y^{\prime}$.

Let us write the equation for the synchronization plane $i_{1} k_{3} j$ in the form

$$
F=A_{3} \Delta x_{i_{1} j}+B_{3} \Delta y_{i_{1} j}+C_{3} \Delta z_{i_{i} j}=0 .
$$


Equation (30.15) corresponds to the correction equation in the form

$$
A_{3} d x_{j}+D_{3} d y_{j}+C_{3} d z_{j}+W_{3}=\varepsilon_{3}, \text { weight } p_{3}
$$

where $d x, d y$, and $d z$ are the corrections to the approximate coordinates of point $j ; p_{3}$ is the weight calculated by Formula (29.32), replacing the subscripts $k$ by 3 and $j_{1}$ by $1_{1}$.

The correction equations for angles $\Lambda$ and $\Phi$, which characterize the direction of the chord $i_{2} j$, have the form

$$
\begin{aligned}
& a \cos \Phi d x+b \cos \Phi d y+l_{\Lambda} \cos \Phi=v_{\Lambda} \cos \Phi ; \text { welght } p_{\Lambda} \cos \Phi \\
& c d x+d d y+e d z+l_{\Phi}=v_{\Phi} ; \text { weight } p_{\Phi} .
\end{aligned}
$$

In the assumed coordinate system, the coefficients and weight $/ 135$ of Equation (30.16), as well as the coefficients of Equations $(30.17)$, assume the values

$$
\begin{gathered}
A=0 ; \quad B=0 ; \\
C=\sin \left(\gamma_{j k_{3}}-\gamma_{i_{1} h_{2}}\right)=\sin \left(\psi_{k_{3}} ;\right. \\
p_{3}=\frac{1}{2 D_{i_{1} j} \cos 2 \frac{\Upsilon_{3}}{2}} ; \\
a=0 ; \quad b=\frac{1}{D_{i_{2} j}} ; \quad c=-\frac{\sin \Phi}{L_{i_{3} j}} ; \\
d=0 ; \quad e=\frac{\cos (j)}{D_{i_{2} j}} .
\end{gathered}
$$

By introducing the weights of measurements $p_{\delta}=p_{\gamma} \cos \delta=$ $1 / \mu^{2}=1$ and using the first two expressions of (29.37), we have

$$
\begin{gathered}
p_{\Lambda \cos \Phi}=4 \cos ^{2} \frac{\lambda}{2} \sin 2 \frac{\varphi}{2}, \\
p_{\Phi}=4 \sin ^{2} \frac{\lambda}{2} \sin ^{2} \frac{\varphi}{2} .
\end{gathered}
$$


Finally, the matrices of the equations for the errors $K$ and weights $P$ for the construction considered assume the form

$$
\begin{aligned}
& K=\left[\begin{array}{ccc}
0 & 0 & -\sin \psi_{3} \\
0 & \frac{1}{-D_{i_{2}}} & 0 \\
\frac{\sin \Phi}{D_{i_{2} j} j} & 0 & \frac{\cos \Phi}{D_{i_{2} j}}
\end{array}\right] \text {, } \\
& P=\left[\begin{array}{ccc}
\frac{1}{2 D_{i_{1}}^{2} \cos ^{2}-\frac{\varphi}{2}} & 0 & 0 \\
0 & 4 \cos ^{2} \frac{\lambda}{2} \sin ^{2} \frac{\varphi_{3}}{2} & 0 \\
0 & 0 & 4 \sin ^{2} \frac{\lambda}{2} \sin ^{2} \frac{\Psi_{3}}{2}
\end{array}\right] .
\end{aligned}
$$

Inverting matrix $\mathrm{K}^{\mathrm{T}} \mathrm{PK}$, we have

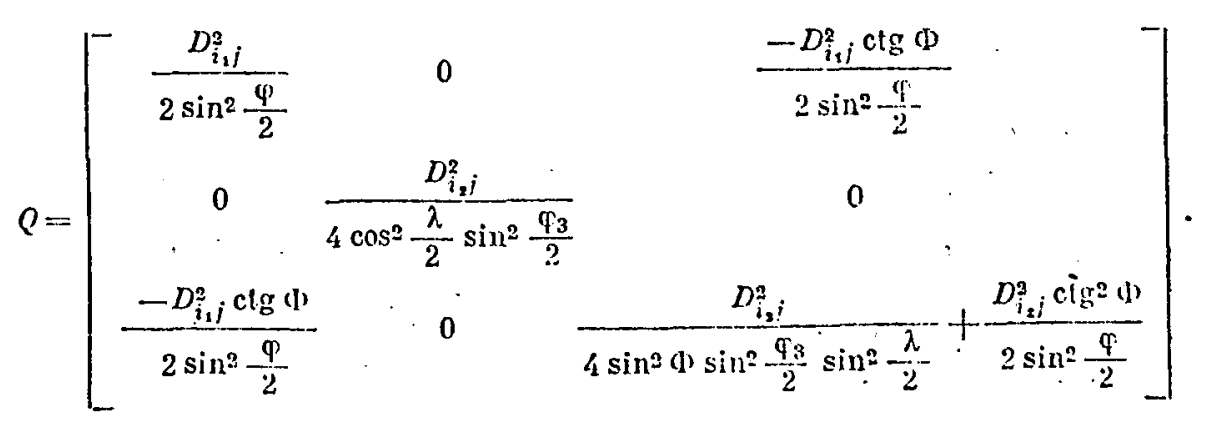

From (30.22) we obtain the expression for the square of the $/ 136$ error in calculating the position of point $J$

$$
\begin{aligned}
& M_{j}^{2}=\mu\left[\frac{D_{i_{1} j}^{3}}{2 \sin ^{2} \frac{\mathscr{T}_{3}}{2}-\sin ^{2} \psi}+\frac{D_{i_{2} i}^{2}}{4 \sin ^{2} \frac{\varphi}{2} \sin ^{2} \cdot \frac{\lambda}{2} \sin ^{2} \psi}+\right. \\
& \left.+\frac{D_{i, j}^{3}}{4 \sin ^{2} \frac{\varphi}{2} \cos ^{2} \frac{\lambda}{2} \sin ^{2} \psi}\right]
\end{aligned}
$$


where $\psi$ is the angle formed by chord $i_{2} j$ with plane $i_{1} k_{3} j$.

The error in the AES position - with synchronous calculation of the direction to it from one observation point and declination from another point, or the error in the observation position in a calculation of the direction from it toward one known AES position and declination of another point - may also be calculated by Formula (30.13). In this case, the error in the position of the plane should be calculated by Expression (29.6). The error in the AES position in this case will be

$$
M_{k}^{2}=\frac{m_{\bar{\beta}}^{2}}{2}\left(\rho_{i_{1}, k}^{2}+\frac{\rho_{i_{1} k}^{2}+\rho_{i_{2} k}^{2}}{\sin ^{2} \psi}\right)
$$

When optical measurements are combined with Doppler measurements, the elementary figure for calculation of the position of the observation point in the direction toward one known AES position and the difference in distances to two known AES positions may also be regarded as intersection of the direction with the plane. In this figure, the surface of the asymptotic cone of the hyperboloid, corresponding to the measured difference in distances, may be regarded as the plane, tangent to the generatrix of the cone, near the point. The error in the position of the point may then be found from Formula (30.15), which in the given case assumes the form

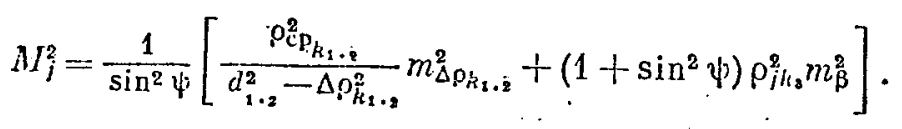

When the direction from the point is measured at one of the two AES positions, to which the distance differences were calculated, we can use the value of the angle $\varphi$ between the measured 
directions to the AES and the direction to the center of the celestial chord in (30.25) instead of angle $\psi$.

Let us consider another method of deriving the formula for the error in the position of the point from the measured difference in distances to two unknown AES positions and observation of $\gamma$ and $\delta$ from it to a single AES position.

Let us select a coordinate system such that plane $k_{1} j k_{2}$ is parallel to plane $\mathrm{z}^{\prime} \mathrm{y}^{\prime}$, and direction $\mathrm{k}_{1} \mathrm{k}_{2}$ is parallel to the coordinate axis oy' (Figure 49).

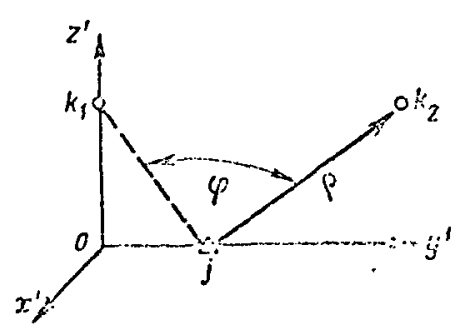

Figure 49.
Let us consider the problem of calculating the position of point $j$, assuming that the position of points $k_{1}$ and $k_{2}$ is known. The matrix of coefficients of the equations for the errors in measured values $(\gamma \cos \delta)^{j k_{2}}$, $\delta_{j k_{2}}$ and $\Delta \rho_{j_{12}}$ in the given construction has the form (subscript $j$ is ommitted for simplicity)

$$
K=\left[\begin{array}{ccc}
\frac{1}{\rho_{2}} & 0 & 0 \\
0 & -\frac{\sin \delta_{2}}{\rho_{2}} & \frac{\cos \delta_{2}}{\rho_{2}} \\
0 & \cos \delta_{2}+\cos \delta_{1} & \sin \delta_{2} \cdots \sin \delta_{1}
\end{array}\right] .
$$

As in all preceding constructions, let us assume that $\mathrm{p}_{\delta}=$ $p_{\gamma} \cos \delta=1$. Then

$$
p_{\Delta \rho}=\frac{\mu^{2}}{m_{\Delta \rho}^{2}}=\frac{\varkappa^{2}}{p_{2}^{2}\left(1-\cos \tau^{2}\right)^{2}}=l^{2}
$$


where $k$ is the ratio of the errors in the angular and linear measurements; and $\varphi$ is the angle of intersection of directions $j k_{1}$ and $j k_{2}$.

Accordingly, the weight matrix assumes the form

$$
p=\left[\begin{array}{lll}
1 & 0 & 0 \\
0 & 1 & 0 \\
0 & 0 & l^{2}
\end{array}\right]
$$

Taking into account the equalities:

$$
\begin{aligned}
\cos \delta_{2}+\cos \delta_{1} & =\cos \delta_{2}(1-\cos \varphi)-\sin \varphi \sin \delta_{2}=m, \\
\sin \delta_{2}-\sin \delta_{1} & =\sin \delta_{2}(1-\cos \varphi)-\sin \varphi \cos \delta_{2}=n,
\end{aligned}
$$

we compile the matrix of coefficients of the normal equations

$$
N=K^{T} P_{K}
$$

and, transforming it, we obtain the matrix of weight coefficients

$$
Q=\left[\begin{array}{ccc}
\rho_{2}^{2} & 0 & 0 \\
0 & \frac{\cos ^{2} \delta_{2}+l^{2} n_{2}^{2} \rho_{2}^{2}}{l^{2}(1-\cos \varphi)^{2}} & -\frac{\sin \delta_{2} \cos \delta_{2}+l^{2} m n_{0}^{2}}{l^{2}(1-\cos \varphi)^{2}} \\
0 & -\frac{\sin \delta_{2} \cdot \cos \delta_{2}+l l^{2} m n_{2}^{2}}{l^{2}(1-\cos \varphi)^{2}} & \frac{\sin ^{2} \delta_{2}+l l^{2} m_{0}^{2} \rho_{2}^{2}}{l^{2}(1-\cos \varphi)^{2}}
\end{array}\right] .
$$

Introducing the error of unit weight $\mu$, from matrix (30.31), we obtain the expression for the square of the error in calculating the position of a point in the given construction

$$
M_{j}^{2}=\mu^{2} \rho_{2}^{2}\left(1+\frac{2}{1-\cos \varphi}\right)+\frac{m_{\Delta \rho}^{2}}{(1-\cos \varphi)^{2}}
$$

Taking into account Formula (30.27), Expression (30.32) is transformed to the form

$$
M y=\mu^{2} \Omega^{3}\left(2+\frac{1}{x^{2}}+\frac{1+\cos \varphi}{1-\cos \varphi}\right)
$$




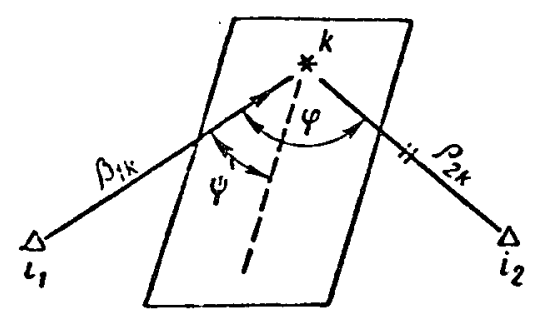

Figure 50.

To estimate the accuracy of all types of three-dimensional linearangular intersection, it is expedient to use angle $\Phi$ between the direction and the line whose length is calculated (Figure 50), instead of angle $\psi$ between the direction and the plane in (30.13).

In this case in Formulas $(30.13)$, sin $\psi$ is replaced by $\cos \varphi / 138$ and Formula (30.13) assumes the form

$$
M^{2}=\frac{m_{H}^{3}}{2}+\frac{\frac{m_{H}^{2}}{2}+m_{p}^{2}}{\cos ^{2} \phi}
$$

Formula (30.34) is applicable for estimation of the accuracy: (1) of the figure for calculation of the AES position by the direction from one point and the distance from another observation point; (2) of the figure for calculating the position of the observation point by the direction from it to one known AES position, and the distance to another point.

In the latter case, $m_{p}$ is calculated from Expression (29.7) and the error in the position of the point will be

$$
H_{j}^{2}=\frac{\rho^{2}}{2} m_{\beta}^{2}+\frac{\frac{\rho^{2}}{2} m_{\dot{p}}^{2}+m_{\mathrm{p}}^{2}}{\cos ^{2} \varphi}
$$

Formula (30.35) may be obtained in a somewhat different form. If angles $\alpha_{1}$ and $\delta_{1}$ in the construction shown in Figure 46 are measured from point $j_{1}$, and the distance $\rho_{2}$ is measured from point $j_{2}$ to the satellite position $k$, then in the assumed coordinate system the matrices of coefficients of the equations for errors $K$ and weights $P$ assume the form 


$$
\begin{array}{r}
K=\left[\begin{array}{ccc}
\frac{1}{\rho_{1}} & : 0 & 0 \\
0 & -\frac{\sin \delta_{1}}{\rho_{1}} & \frac{\cos \delta_{1}}{\rho_{1}} \\
0 & -\cos \delta_{2} & \sin \delta_{2}
\end{array}\right] \\
P=\left[\begin{array}{ccc}
1 & 0 & 0 \\
0 & 1 & 0 \\
1 & 0 & \frac{x^{2}}{\rho_{2}^{2}}
\end{array}\right]
\end{array}
$$

Inverting the matrix of normal equations

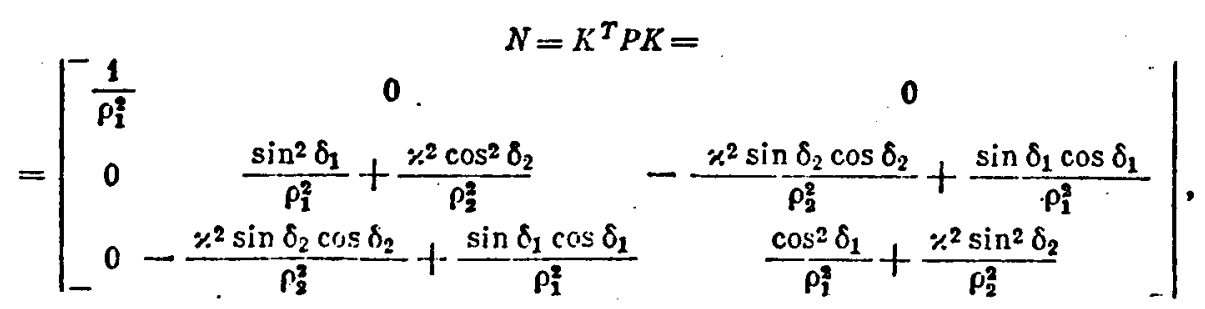

we obtain the matrix of coefficients, from which it follows that the error in calculating the position of the point (if the AES positions are known) or of the satellite (if the positions of the points are known) is equal to:

$$
M^{2}=\mu\left(\mu_{1}^{2}+\frac{\varkappa_{2}^{2} \rho_{1}^{2}+r_{2}^{2}}{\varkappa^{2} \cos ^{2} q^{2}}\right)
$$

where $k$ is the error ratio of the angular and linear measurements.

Formula (30.34) is also applicable for analysis of the accur-1139 acy of the AES position, calculated by the direction to it from one point and by the difference in distances to it and another known AES position from another point. In the latter case, $m_{p}$ is calculated by Expression (29.11) and the error in the AES position is

$$
M_{k}^{2}=\frac{\rho^{3}}{2} m_{\dot{p}}^{2}+\frac{\frac{\rho^{2}}{2} m_{\xi}^{2}+\frac{d^{2} l^{2}}{\Delta \rho^{2}} m_{\Delta \rho}^{2}}{\cos ^{2} \varphi} .
$$


If the direction and distance are calculated from one starting point (polar intersection), angle $\varphi$ vanishes $\left(\psi=90^{\circ}\right)$, and Formula (30.34) assumes the form

$$
M^{2}=m_{\mathrm{H}}^{2}+\mathrm{m}_{\mathrm{p}}^{2}
$$

Expression (30.40) is used for estimating the accuracy of the AES position or the observation point by the direction measured from the point and by the distance to the AES position.

In this case the error formula assumes the form

$$
M_{j}^{2}=\dot{j}_{j k}^{2} m_{\dot{p}}^{2}+m_{\mathrm{p}}^{2}
$$

For this same case, from Expression (30.38) we obtain

$$
M_{j}=\mu \rho \sqrt{2+\frac{1}{k^{2}}}
$$

and, when the relative errors in measurement of the distance and angular values are equal,

$$
M_{i}=\mu \rho \sqrt{3}
$$

When calculating the AES position by the direction to it and by the difference in distances to it and another (known) AES position from the same point, the error formula for the AES position, on the basis of (30.39) will be

$$
M P_{h}^{2}=\rho^{2} m_{\beta}^{2}+\frac{d ? l 2}{\Delta \rho^{4}} m_{\Delta \rho}^{2} .
$$


When calculating the position of the point by the direction and base of the chord, the error formula for the position of the point will be

$$
M 1_{i}^{2}=m_{B}^{B} D^{2}+m_{D}^{2}
$$

in which the expression for $\mathrm{m}_{\mathrm{B}}$ is taken from (29.27) or (29.28), and $m_{D}$ is calculated by Expression (29.45) when measuring the distance to the AES, or by (29.46) when measuring the difference in distances.

Formula (30.13) may be used to estimate the accuracy of the position of the point of intersection of three planes, if this figure is considered as the intersection of one of three planes with the line of intersection of two others. Then, for intersection of three synchronization planes, each passing through the calculated and one of the starting points, the error in the position of the point is described by the expression

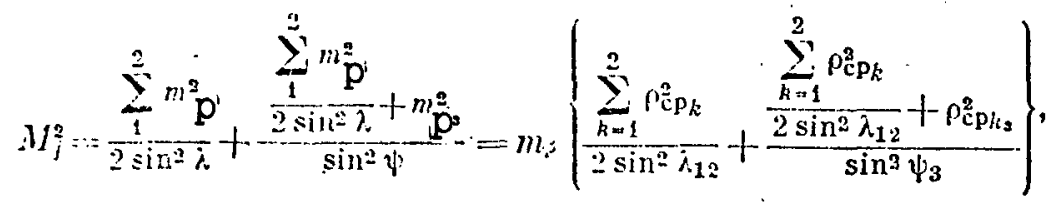

where $\lambda$ is the angle between the synchronization planes, formed by AES positions $k_{1}$ and $k_{2}$, and $\psi_{3}$ is the angle between the line of intersection of these planes and the synchronization plane, formed by the third AES position $\left(k_{3}\right)$.

Cases are possible when, due to the absence of precise time at the observation point, only the declinations of the three known AES positions, rather than the directions from it, will be calculated. Then, taking into account (29.6), the expression for the error in the position of the point will assume the form 


$$
M H_{j}^{2}=\frac{m_{j}^{2}}{2}\left[\frac{\rho_{j k_{1}}^{2}+\rho_{j k_{2}}^{2}}{2 \sin ^{2} \lambda}+\frac{\frac{\rho_{j k_{1}}^{2}+\rho_{j k_{2}}^{2}}{2 \sin ^{2} \lambda}+\rho_{j h_{3}}^{2}}{\sin ^{2} \varphi}\right] .
$$

Similarly, when calculating the position of the point by three differences in distances, calculated from this point to three pairs of known AES positions, taking into account Expression (29.20) and assuming the relative errors in the differences in distances to be equal, we obtain

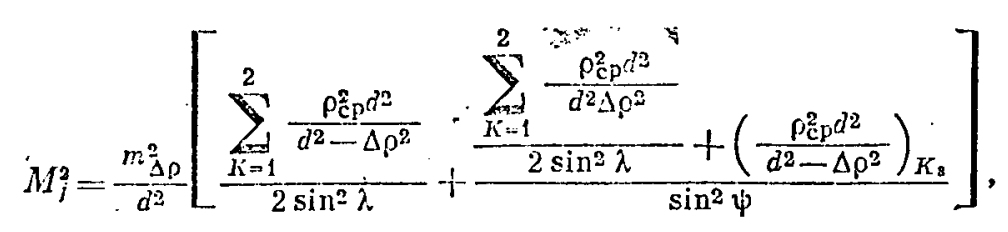

where $K$ is the number of the pair of AES positions to which $\Delta \rho$ is measured.

In the latter two cases, $\lambda$ is the angle between two planes, tangent to the generatrix of two conical surfaces, and $\psi$ is the angle between the line of intersection of these planes (or tangent to the line of intersection of the conical surfaces near the point to be calculated) and a third plane, tangent to the generatrix of the third conical surface.

The same Formula (30.13) is applicable for analysis of the accuracy of three-dimensional linear intersection in cases when the point or the AES position is calculated by the three measured distances or when the AES position is calculated by the three lengths of the focal radil.

If we assume that the relative errors in-measurement of distances are equal, Formula (30.13) will assume the form 


$$
M_{j}^{2}=\frac{m_{\psi}^{2}}{\rho^{2}}\left[\frac{\rho_{1}^{2}+\rho_{2}^{2}}{2 \sin ^{2} \rho_{12}}+\frac{\frac{\rho_{1}^{2}+\rho_{2}^{2}}{2 \sin ^{2} \varphi_{12}^{2}}+\rho_{3}^{2}}{\sin ^{2} \psi}\right],
$$

where $\varphi_{12}$ is the angle between two lines by which the distances are measured; and $\psi_{3}$ is the angle formed by the third line with the plane passing through the first two lines.

When using the focal radil, $m_{\rho}$ are calculated by Formula $(29.11)$.

The Error in the Vertex of Instersection of Three Planes

As was shown, intersection of three planes may be regarded as intersection of one of the planes with the direction of the line of intersection of two others, and for estimating the accuracy of calculating the position of the point of intersection, Formula (30.46) may be used.

However, it is expedient to consider another derivation of the formula for the error in the point of intersection of the three planes.

The coordinates of the intersection point are found from solution of the system of equations for three planes

$$
\begin{aligned}
& A_{1} X+B_{1} Y+C_{1} Z-D_{1}=0 \\
& A_{2} X+B_{2} Y+C_{2} Z-D_{2}=0 \\
& A_{3} X+B_{3} Y+C_{3} Z-D_{3}=0
\end{aligned}
$$

or after transfer of the origin to the point to be determined and normalization of the coefficients and the free terms of equations

$$
\begin{aligned}
& A_{1}^{\prime} X^{\prime}+B B_{1}^{\prime} Y^{\prime}+C_{1}^{\prime} Z^{\prime}-d_{1}=0 \\
& A_{2}^{\prime} X^{\prime}+B B_{2}^{\prime} Y^{\prime}+C_{2}^{\prime} Z^{\prime}-d_{2}=0 \\
& A_{3}^{\prime} X^{\prime}+B_{3}^{\prime} Y^{\prime}+C_{3}^{\prime} Z^{\prime}-d_{3}=0 .
\end{aligned}
$$


In Formulas (30.51) the normalized free term is the error in construction of the given plane. Its mean square value is calculated by Formula (29.16) (for $m_{p}$ ). In matrix form, Expressions (30.51) are written

$$
A X=d
$$

Hence,

$$
X=A^{-1} d
$$

and, according to $(27.1)$

$$
M_{X}^{2}=\left(A^{-1}\right) \mathrm{M}_{\mathrm{p}}^{2}\left(A^{-1}\right)^{T} .
$$

In expanded form $(30.54)$ is written

$$
M_{X}^{2}=\frac{1}{\Delta^{2}}\left[\begin{array}{ccc}
L_{1} & L_{2} & L_{3} \\
N_{1} & M_{2} & M_{3} \\
N_{1} & N_{2} & N_{3}
\end{array}\right] \cdot\left[\begin{array}{ccc}
m_{\hat{p}^{3}}^{\mathrm{g}} & 0 & 0 \\
0 & m_{\mathrm{p}^{2}}^{2} & 0 \\
0 & 0 & m_{\mathrm{p}^{3}}^{2}
\end{array}\right] \cdot\left[\begin{array}{rrr}
L_{1} & M_{1} & N_{1} \\
L_{2} & M_{2} & N_{2} \\
I_{3} & M_{3} & N_{3}
\end{array}\right],
$$

where $m_{p_{1}}$ is the mean square error in the construction of the given plane; $L_{1}, M_{1}$, and $N_{1}$ are the direction cosines of the lines of Intersection of planes $Q_{2}$ and $Q_{3}, Q_{1}$ and $Q_{3}, Q_{1}$ and $Q_{2}$ respectively; and $\Delta$ is the determinant of system (30.51).

Let us expand $\Delta$ with respect to the elements of the first row

$$
\begin{aligned}
& \Delta=A_{1}^{\prime}\left|\begin{array}{ll}
B_{2}^{\prime} & C_{2}^{\prime} \\
B_{3}^{\prime} & C_{3}^{\prime}
\end{array}\right|+B_{1}^{\prime}\left|\begin{array}{cc}
C_{2}^{\prime} & A_{2}^{\prime} \\
C_{3}^{\prime} & A_{3}^{\prime}
\end{array}\right|+C_{1}^{\prime}\left|\begin{array}{cc}
A_{2}^{\prime} & B_{2}^{\prime} \\
A_{3}^{\prime} & B_{3}^{\prime}
\end{array}\right|== \\
= & A_{1}^{\prime} L_{1}+B_{1}^{\prime} M_{1}+C_{1}^{\prime} N_{1}=\frac{A_{1}^{\prime} L_{1}^{\prime}+B_{1}^{\prime} M_{1}^{\prime}+C_{1}^{\prime} N_{1}^{\prime}}{\hat{\lambda}_{1}}=\frac{\sin \psi_{1}}{\lambda_{1}},
\end{aligned}
$$

where $\lambda_{1}$ is the normalizing factor

$$
\lambda_{1}=\frac{1}{l^{\prime} \overline{L_{1}^{2}+\lambda M_{1}^{2}+N_{1}^{2}}}
$$


$\psi$ is the angle between plane $Q_{1}$ and the Iine of intersection of the other two planes $Q_{2}$ and $Q_{3}$. If the determinant $\Delta$ is expanded with respect to the elements of the second and third rows, similarly, we will have

$$
\Delta=\frac{\sin \psi_{2}}{i_{-2}}=\frac{\sin \psi_{3}}{i_{-3}}
$$

After multiplication of the matrices contained in (30.55), we obtain the following expressions for the diagonal elements of $\operatorname{matrix} \mathrm{M}^{2} \mathrm{X}$ :

$$
\begin{aligned}
& m_{\lambda^{\prime}}^{2}=\frac{1}{\Delta^{2}}\left(\frac{L_{1}^{\prime 2}}{\lambda_{1}^{2}} m_{\mathrm{p}_{\mathrm{p}}}^{2}+\frac{L_{2}^{\prime 2}}{\lambda_{2}^{2}} m_{\mathrm{p}_{2}^{2}}^{2}+\frac{L_{3}^{\prime 2}}{\lambda_{3}^{3}} m_{\mathrm{p}_{1}^{2}}^{2}\right),
\end{aligned}
$$

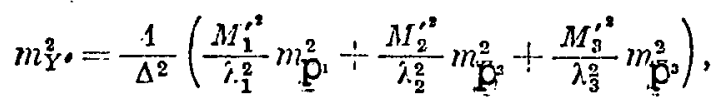

$$
\begin{aligned}
& m_{\bar{L}^{\prime}}^{2}=\frac{1}{\Delta^{2}}\left(\frac{N_{2}^{\prime 2}}{\lambda_{1}^{2}} m_{\underline{p}^{1}}^{2}+\frac{N_{2}^{\prime 2}}{\lambda_{2}^{2}} m_{\ddot{p}^{2}}^{2}+\frac{N_{3}^{\prime}}{\lambda_{3}^{2}} m_{\dot{p}^{3}}^{2}\right) \text {. }
\end{aligned}
$$

Hence, taking the fact into account that $L^{\prime}{ }_{1}^{2}+M_{1}^{\prime}{ }_{1}^{2}+N_{1}^{\prime}{ }^{2}=$ 1 , and also Formulas (30.56) and (30.58), we obtain the final expression for the error in the position of the vertex of intersection of the three planes as a function of the measurement errors and the geometric characteristics of the given intersection

$$
M^{2}=m_{X^{\prime}}^{2}+m_{Y^{\prime}}^{2}+m_{Z^{\prime}}^{2}=\frac{m^{2} p_{i}}{\sin ^{2} \psi_{1}}+\frac{m^{2} p_{2}}{\sin ^{2} \psi_{2}}+\frac{m^{2} \bar{p}_{3}}{\sin ^{2} \psi_{3}}=\sum_{i=1}^{8} \frac{m^{2} p_{1}}{\sin ^{2} \psi_{i}}
$$

Formula (30.60) is used for analysis of the accuracy of the vertex of several elementary figures which we considered above, first, for analysis of the accuracy of the position of the observation point, calculated at the intersection of three synchronization planes. In this case $m_{p}$ is calculated by Formula (29.16) or (29.17) and the expression for the error in the position of the point will be 


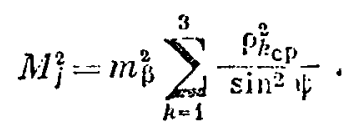

Further, Formula (30.60) may be used to estimate the accuracy of elementary figures which are the intersection of three conical surfaces considered near the point to be calculated, as planes tangent to the generatrix of these conlcal surfaces. Such figures occur when the position of the point is calculated by the declinations of three known AES positions measured on it or by the differences in distances measured from it to three pairs of AES positions. In the first case $m_{p}$ is calculated by Expression (29.6), and $\psi$ is the angle between the direction from the point to one AES position and the plane passing through the direction from the point to two other AES positions. In the second case $\mathrm{m}_{\mathrm{p}}$ is calculated by Expression (29.20), and $\psi$ is the angle between the line connecting the point with the center of the celestial chord between one pair of AES positions (the difference in distances to which is measured) and the plane passing through the lines connecting the point with the center of the celestial chords of two other pairs of AES positions.

The expression for the error in the position of the point in the latter case has the form

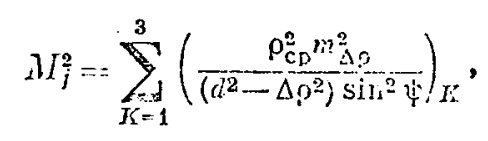

where $K$ is the number of the pair of AES positions.

Formula (30.60) may be used for a priori estimation of the accuracy of the position of the vertex of all types of threedimensional linear intersection in space. Such intersections include figures which occur upon calculation of the AES position 
by the distances measured to it from three observation points, upon calculation of the position of the point by the distances measured from it to three known AES positions, as well as upon calculation of AES positions by the differences in distances measured from the observation points to them, and at the same time - from the lengths of the focal radil of the hyperboloids.

The value of $\mathrm{m}_{\mathrm{p}}$ in Expression (30.60) in the first two cases is calculated by Formula (29.7), and in the third case - by Formula (29.11).

The quantity $\psi$ in these figures is the angle between one line whose length is measured and the plane passing through two other lines (one focal radius and the plane passing through two other radii).

Thus, for example, the error in calculating the position of a point by the distances measured from it to three known AES positions will be calculated by the formula

$$
M_{j}^{a}=\sum_{k=1}^{3} \frac{m_{f k}^{2}}{\sin ^{2}+k}
$$

31. The Effect of Errors in the Position of Starting Points

The effect of systematic errors in initial data, which cause a general shift of the entire triangulation or in the presence of more than one starting point - a general shift, rotation and an error in the scale of space triangulation is well known.

We have given approximate consideration below to the effect of random errors in the position of starting points and this effect on the previously derived formulas for an a priori estimation of the accuracy of the elements and figures of space triangulation. 


\section{Effect of Errors in Starting Points on Errors in}

Triangulation Elements

An error in the position of the point in a three-dimensional coordinate system may be represented by the expression

$$
M_{i}^{2}=m_{X}^{2}+m_{Y}^{2}+m_{Z}^{2}
$$

If the components of errors on the coordinate axes are assumed to be equal, we may assume that, independently of the direction of the coordinate axes, we have

$$
M_{i}^{2} \doteq 3 m^{2}
$$

On this basis, the error component of the starting point position in a direction moving away from this point, and, consequently, the effect of the error in the position of the point at a distance measured from it, or on the plane passing through this point, will be

$$
\mathrm{m}_{\mathrm{p}}^{2}=\frac{M_{i}^{2}}{2}
$$

The error component of the position of the starting point in the plane normal to the difrection passing from the point, and consequentiy, the effect of the error on displacement of the direction pasing through the point, will be

$$
m_{\mathrm{if}}^{2}=\frac{2}{3} M_{i}^{2}
$$


We should especlally discuss the effect of errors in the position of starting points on the errors in space triangulation elements, obtained as a result of measuring the differences in distances.

The error in the length of the focal radius depends on the distance between the observation point and the plane passing through the center of the celestial chord $(2)$, i.e., on the displacement along one of the coordinate axes. Having differentiated Expression (29.9) with respect to $Z$ and converting to mean square errors, assuming that $\mathrm{m}^{2}{ }_{l}=\mathrm{M}^{2}{ }_{1} / 3$, we obtain the error in the length of the focal radius due to the effect of the error in the position of the point

$$
m_{p}^{2}=\frac{d^{2}}{3 \Lambda \varphi^{2}} M_{i}^{2}
$$

The characteristic of the hyperboloid is the angle $\varphi^{\prime}$ between the surface of the asymptotic cone and the axis of the hyperbolold (see Figure 42). It is obvious from Formula (29.18) that angle $\varphi^{\prime}$ depends on the length of the celestlal chord $d$.

Having differentiated (29.18) with respect to d, substituting It into (29.19) and converting to mean square errors, assuming that $\mathrm{m}^{2}{ }_{\mathrm{d}}=2 / 3 \mathrm{M}_{\mathrm{k}}{ }_{\mathrm{k}}$, we obtain the error in the position of the plane, with which we approximate the asymptotic cone, caused by the effect of the error in the length of the celestial chord,

$$
\mathrm{m}_{\mathrm{p}}^{2}=\frac{2 \rho_{\mathrm{p}}^{2} \Delta \rho^{2}}{3 d^{2}\left(d^{2}-\Delta \rho^{2}\right)} M_{h}^{2}
$$


The effect of the errors in AES positions, which are the focl of the hyperbolold, may also be reflected in rotation of the axis of the hyperboloid, which imparts an additional displacement to the hyperboloid surface, equal to

$$
\mathrm{m}_{\mathrm{p}}^{2}=\frac{2 \rho_{\mathrm{c}}^{2} \mathrm{p}}{3 d^{2}} M_{k}^{2}
$$

The total effect of errors in the AES position on the error in the position of the hyperboloid will be

$$
\mathrm{m}_{\mathrm{p}}^{2}=\frac{2 \rho_{\mathrm{c}}^{2} \mathrm{p}}{3\left(d^{2}-\Delta \rho^{2}\right)} M_{k}^{2}
$$

Moreover, we should remember that, since the vertex of the cone is not the AES position, but the center of the celestial chord, the total displacement of the surface will be

$$
\mathrm{m}_{\mathrm{p}}^{2}=\left(\frac{2 o_{\mathrm{p}}^{2} \mathrm{p}}{d^{2}-\Delta \rho^{2}}+\frac{1}{2}\right) \frac{M_{k}^{2}}{3}
$$

Effect of Errors in Starting Points on Errors in the Position of the Vertices of Elementary Figures

For the main types of elementary figures considered previously, the effect of errors in the position of starting points on the position of the calculated points may be represented in the following manner.

For three-dimensional angular intersection

$$
M^{2}=\frac{1}{3}\left(\frac{M_{1}^{2} M_{2}^{2}}{M_{1}^{2}+M_{2}^{2}}+\frac{M_{1}^{2}+M_{2}^{2}}{\sin ^{2} \varphi}\right) \cong \frac{1}{3}\left(\frac{1}{4}+\frac{1}{\sin ^{2} \varphi}\right) \sum_{i=1}^{2} M_{i}^{2}
$$

or with equaliy precise initial data 


$$
M^{2}=\frac{2 M_{l}^{2}}{3 \sin ^{2} \varphi}
$$

For intersection of the direction with the plane

$$
M^{2}=-\frac{1}{3}\left(M_{i_{2}}^{2}+\frac{M_{i_{2}}^{2}+M_{i_{1}}^{2}}{\sin ^{2} \psi}\right)
$$

or

$$
M^{2}=\left(1+\frac{2}{\sin ^{2} \psi}\right) \frac{M_{i}^{2}}{3}
$$

For linear-angular intersection

$$
M^{2}=\frac{1}{3}\left(M_{i_{2}}^{2}+\frac{M_{i_{2}}^{2}+M_{i_{1}}^{2}}{\cos ^{2} \varphi}\right)
$$

or

$$
M^{2}=-\left(1+\frac{2}{\cos ^{2} \varphi}\right) \frac{M_{i}^{2}}{3}
$$

To calculate the position of the point by the polar method.

$$
M^{2}=M_{i}^{2}
$$

For intersection of three planes or three-dimensional inear Intersection

$$
M^{2}=\frac{1}{3} \sum_{i=1}^{3} \frac{M_{i}^{2}}{\sin ^{2} \psi_{i}}
$$

or

$$
M^{2}=\frac{M_{i}^{2}}{3} \sum_{i=1}^{3} \frac{1}{\sin ^{2} \psi_{i}}
$$


Taking into account the dependence of the previously obtained errors in the elements of space triangulation, constructed by using the measured differences in distances, on the errors in initial data (31.5) and (31.8), we reduce the formulas of the total effect of errors in the initial data on the accuracy of the two main types of elementary figures of such triangulation.

For a figure for calculating the AES position by the direction to it from one point and the difference in distances to it and another AES position from another point, we have

$$
M_{R}^{2}=\frac{M_{i}^{2}}{3 \cos ^{2} \varphi}\left(2+\cos ^{2} \varphi+\frac{d^{2}}{\Delta \rho^{2}}\right) .
$$

For a figure for calculating the position of the point by the difference in distances from it to two known AES positions and by the direction to the third known AES position, we have

$$
M_{j}^{2}=\frac{M_{k}^{2}}{3 \sin ^{2} \psi}\left(\frac{3}{2}+\sin ^{2} \psi+\frac{2 \rho_{\mathrm{cp}}^{2}}{d^{2}-\Delta \rho^{2}}\right)
$$

32. Combining the Formulas for A Priorlestimation of the Accuracy of the Elements and Elementary Figures of Space Triangulation

For convenience in using the formulas for a priori estimation of accuracy, let us reduce them to a single table for a further analysis.

For 1llustration, the main figures in Table 1 are represented by diagrammatic drawings with the following notations: 
$\square$ - starting point;

$\Delta$ - calculated point;

O - known AES position;

* - calculated AES position;

$\rightarrow^{*}$ - direction from the point to the AES position;

...** - declination of AES position;

$-1-$ - $^{*}$ - distance from the point to the AES position;

.... $\left\{_{*}^{*}\right.$ - difference in distances from the point to two AES positions;

$\rightarrow-\rightarrow$ direction of the chord;

$--1-1--$ - length of the chord.

In Table 1 the formulas for a priori estimation of the accuracy of the elements and figures are first presented only for photographic measurements, then for combinations of photographic observations and measured distances, and for combinations of photographic observations and the differences in distances.

33. The Effect of the Number of Measurements on the Results of A Priori Estimation of the Accuracy of Space Triangulation Figures

We call the figures containing the minimum number of measurements required to obtain a result - for calculation of the position of the point or the value of some space triangulation element - an elementary figure. 


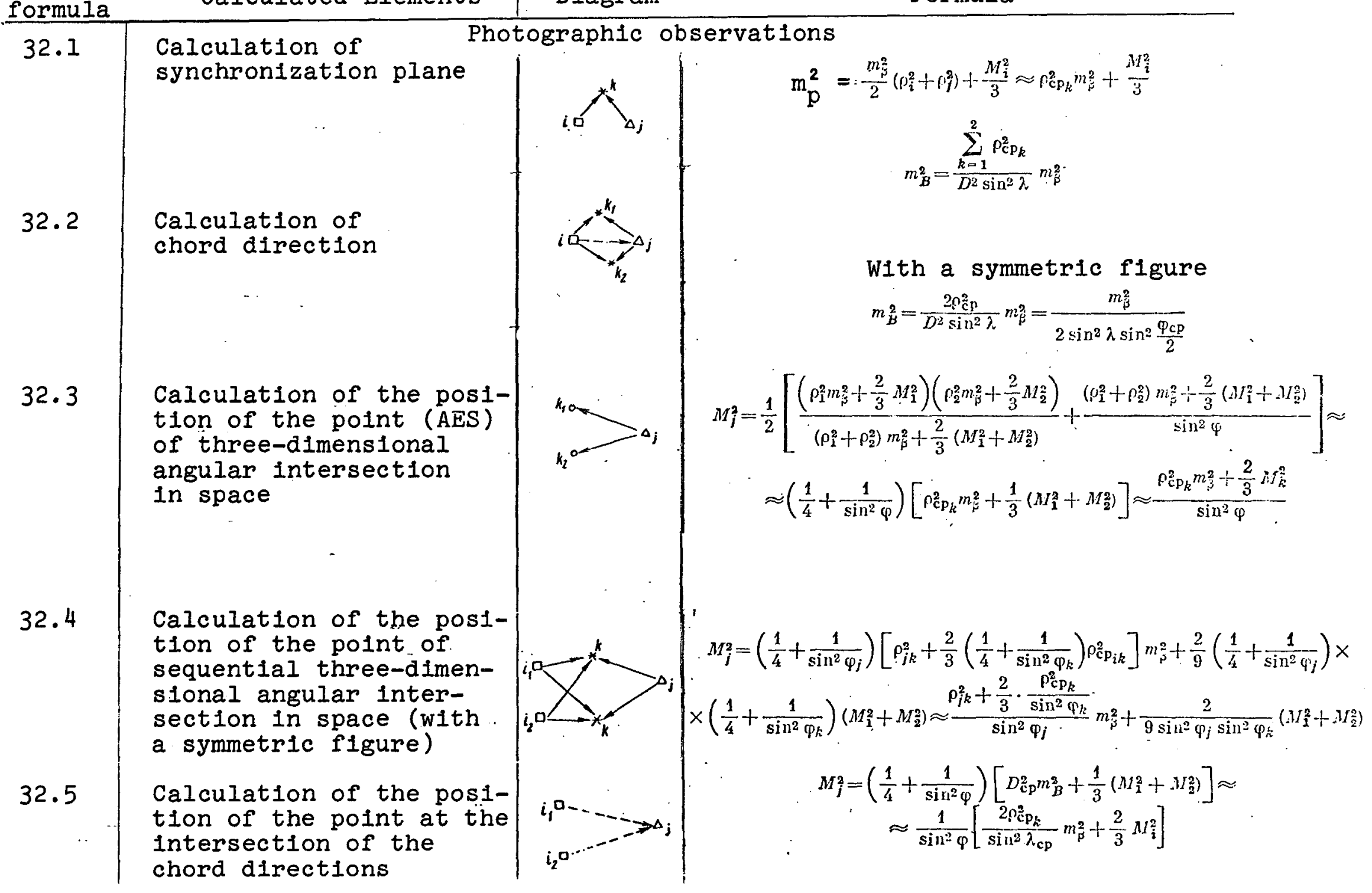
chord directions 
TABLE 1 (Continued)

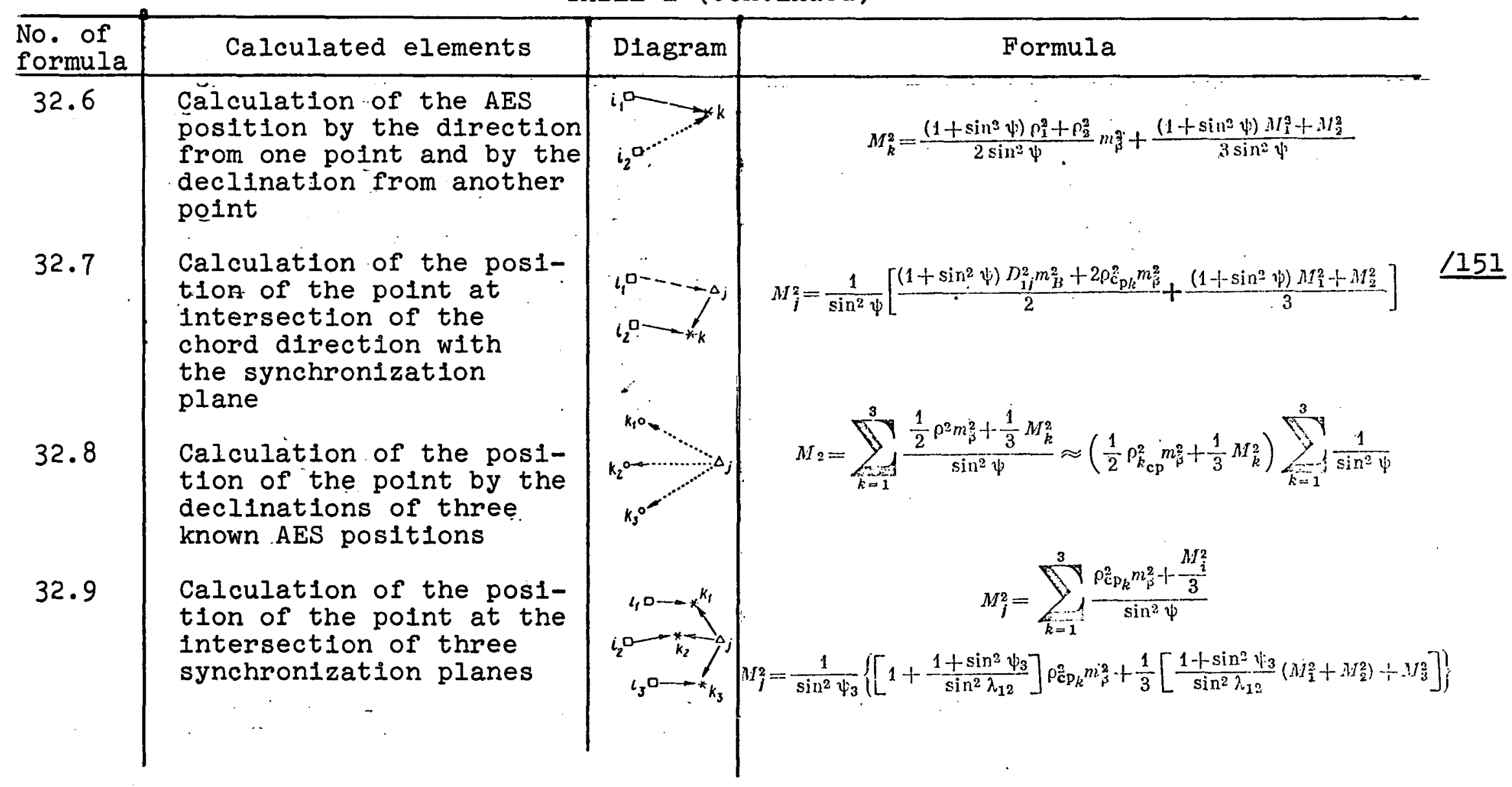

Combinations of photographic observations with distance measurement

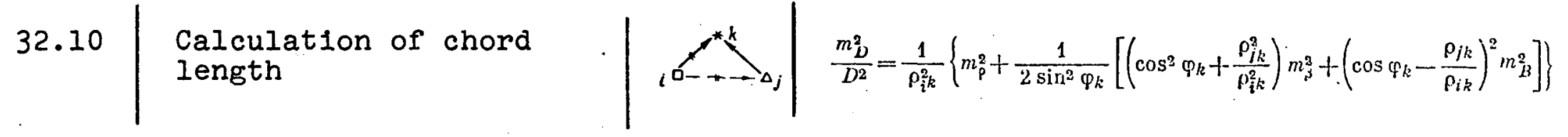


TABLE 1 (Continued)

$N$

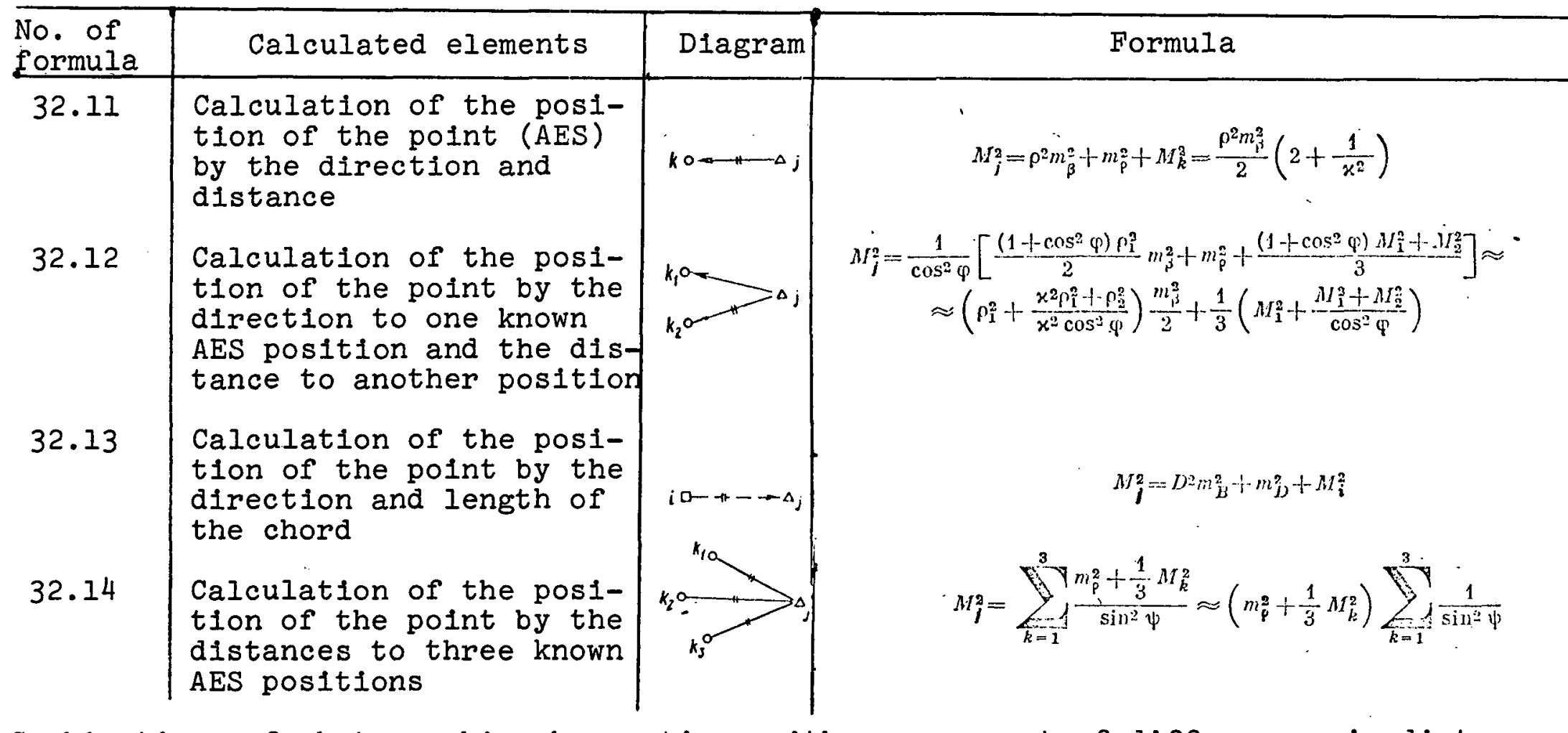

Combinations of photographic observations with measurement of differences in distances

\begin{tabular}{|c|c|}
\hline 32.15 & $\begin{array}{l}\text { Calculation of chord } \\
\text { length }\end{array}$ \\
\hline 2.16 & $\begin{array}{l}\text { Calculation of the AES } \\
\text { position by the direc- } \\
\text { tion from one point and } \\
\text { by the difference in } \\
\text { distances from another } \\
\text { point }\end{array}$ \\
\hline
\end{tabular}


TABLE I (Continued)

\begin{tabular}{|c|c|c|c|}
\hline $\begin{array}{l}\text { No. of } \\
\text { formula }\end{array}$ & Calculated elements & Diagram & Formula \\
\hline 32.17 & $\begin{array}{l}\text { Calculation of the AES } \\
\text { position by the direc- } \\
\text { tion and difference in } \\
\text { distances }\end{array}$ & $i^{0}$ & $M_{k_{1}}^{2}=\rho^{2} m_{\beta}^{2}+\frac{d^{2} l^{2}}{\Delta \rho^{4}} m_{\Delta \varphi}^{2}+\left(1+\frac{d^{2}}{3 \Delta \rho^{2}}\right) M_{i}^{2}$ \\
\hline 32.18 & $\begin{array}{l}\text { Calculation of the posi- } \\
\text { tion of the point by the } \\
\text { difference in distances } \\
\text { to two known AES posi- } \\
\text { tions and by the direction } \\
\text { to the third position }\end{array}$ & & $I_{j}^{2}=\frac{1}{\sin ^{2} \psi}\left[\frac{\sin ^{2} \psi+1}{2} \rho_{6}^{2} m_{j}^{2}+\frac{\rho_{\mathrm{c}}^{2} \mathrm{p}_{1,2}}{d_{12}^{2}-\Delta \rho_{j}^{2}} m_{\Delta \rho}^{2}+\frac{1}{3}\left(\frac{3}{2}+\sin ^{2} \psi+\frac{2 \rho_{c}^{2} p_{1,2}}{d_{12}^{2}-1 \rho^{2}}\right) y_{k}^{2}\right]$ \\
\hline 32.19 & $\begin{array}{l}\text { Calculation of the posi- } \\
\text { tion of the point by the } \\
\text { difference in distances } \\
\text { to two known AES positions } \\
\text { and by the direction to } \\
\text { one of them }\end{array}$ & & 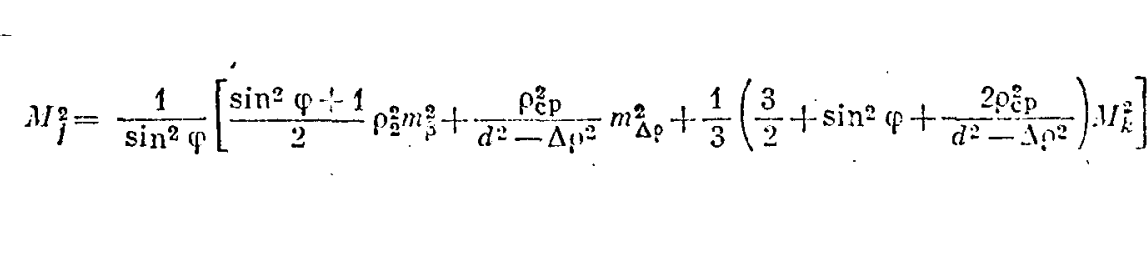 \\
\hline 32.20 & $\begin{array}{l}\text { Calculation of the posi- } \\
\text { tion of the point by the } \\
\text { differences in the dis- } \\
\text { tances to three pairs of } \\
\text { known AES positions. }\end{array}$ & $k_{3} O$ & 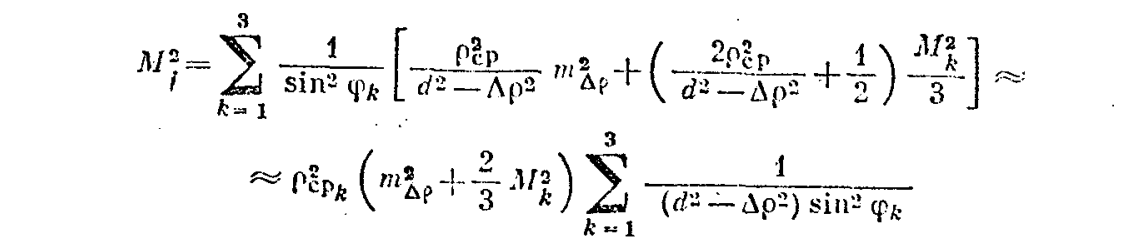 \\
\hline
\end{tabular}


The appearance of excess measurements in the figure should lead to variation of the previously derived formulas for estimation of accuracy. As we know, excess measurements in all cases increase the accuracy of the result, but the effect of an increase in the number of measurements may be different.

In order to establish the effect of excess measurements on the formulas for an a priori estimation of the accuracy of elementary figures, let us present an expression for the error in the elementary figures in general form as

$$
M_{e}^{2}=\sum Q_{i} m_{i}^{2}
$$

where $m_{1}$ are the errors in measurements of the elements of the figures and $Q_{1}=1 / p_{1}$ are the weight coefficients.

Let us consider an increase in the number of measurements of elements of the figure without variation of its geometric shape.

If some element of the figure is measured repeatedly, assuming that there are no systematic errors in the measurements, the error of the calculated measurement may be

$$
m^{2}=\frac{1}{\sum_{\substack{1 \\ 1}}^{n} \frac{1}{m_{e}^{2}}},
$$

where $\mathrm{n}$ is the number of measurements; and $\mathbb{M}_{e}$ is the error in single measurement of the element.

If the measurements are equally precise, then

$$
m^{2}=\frac{m_{e}^{2}}{n} \text {. }
$$


It is more complex to calculate the effect of excess measure-/154 ments, which vary the geometric shape of the elementary figure.

Most of the figures which we considered are intersections, and their excess measured elements are geometrically interrelated in different ways. Since consideration of the effect of element errors on variation of expressions for the errors of elementary figures is rather complex, we will consider the simplest cases here.

If the elementary figure for calculation of a chord is considered as the intersection of two planes, and intersection of three planes is reduced to intersection of the direction with the plane, then, along with the figure for the intersection of the direction with the plane and three-dimensional angular intersection, all these elementary figures are formed by two elements, and should contain a term of the following form in the formulas for a priori analysis of accuracy

$$
M_{e}^{2}=Q\left(m_{i_{1}}^{3}+m_{i_{2}}^{2}\right)
$$

where $Q=1 / \sin ^{2} \psi$ characterizes the geometric relationship between the measured elements.

An Increase in the number of measurements above the two required to a number $n$ increases the number of angles between the pairs of measured elements, which becomes equal to the $2\left(c_{n}^{2}\right)$ number of combinations, out of $n$.

To obtain the approximate expression for estimating the accuracy of such a non-elementary figure, let us use the formula obtained by Professor K. L. Provorov [44], for the error in multiple angular intersection 


$$
M^{2}=\frac{\sum_{i}^{n} \frac{1}{m_{e}^{2}}}{\sum_{1}^{n} \frac{\sin ^{2} \psi_{e_{1} e_{2}}}{m_{e_{1}}^{2} m_{e_{2}}^{2}}}
$$

or with equally precise measurements

$$
M^{2}=\frac{n m_{e}^{2}}{\sum_{1}^{n} \sin ^{2} \Psi_{\ell_{1} e_{2}}}
$$

On the basis of Formula (33.5), we find the expression for estimation of accuracy for some basic space triangulation figures containing an excess number of measurements.

Error in Chord Direction

Usually, as a result of prolonged observations of space triangulation from each pair of points - the ends of the chord - a rather large number of AES positions will be observed synchronously, and the direction of the chord will be calculated at the intersection of $\mathrm{n}$ synchronization planes.

The error in the direction may be represented by the expression

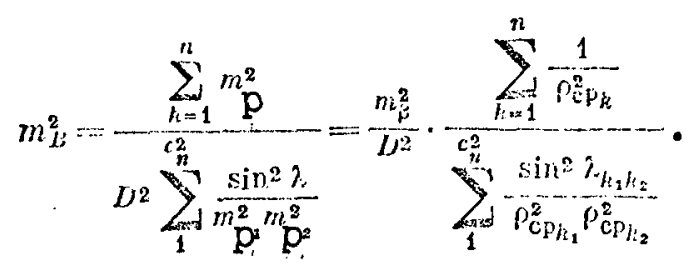


In the case when the average distances are similar, and the synchronization planes corresponding to the AES positions are uniformly located around the chord within the limits of the maximum angle between their extreme positions, equal to $\Omega$,

$$
m_{B}^{2}=\frac{n \rho_{\mathrm{C}_{k}}^{2} m_{E}^{2}}{D^{2} \sum_{c=1}^{n-1}(n-c) \sin ^{2}\left(\frac{c \Omega}{n-1}\right)} .
$$

We may present one more simple approximation expression for the error in the chord direction, derived by Lambeck [57] for uniform distribution of the observed AES positions with respect to the center of the chord

$$
m_{B}^{2}=\frac{m_{p}^{2}}{0,17 n} .
$$

\section{Error in Chord Length}

We previously considered the elementary figures for calculation of chord length, in which the measured linear value was the distance from one of the points (32.10) or the difference in distances from one of the points (32.15).

Measurements of the distances and differences in distances from both points - the chord ends - are essentially possibie.

Then, upon measurement of the distances, the error in the chord length will be calculated by the expression 


$$
\begin{aligned}
& \frac{m_{D}^{2}}{D^{2}}=\frac{m_{\rho}^{2}}{\rho_{1 k}^{2}+\rho_{2 / k}^{2}}+\frac{1}{\sin ^{2} \varphi_{k}}\left[\frac{1+\cos ^{4} \varphi_{k}+\cos ^{2} \varphi_{k} \frac{\rho_{1 k}^{4}-\rho_{2}^{4}}{\rho_{k}^{2} \rho_{2 k}^{2}}}{2 \cos ^{2} \varphi_{k}+\frac{\rho_{1 k}^{4}+\rho_{2 k}^{4}}{\rho_{1}^{2} k \rho_{2 k}^{2}}} m_{j}^{2}-\dot{-}\right. \\
& \left.+\frac{\left(\cos \varphi_{k}-\frac{\rho_{1 k}}{\rho_{2 k}}\right)^{2}\left(\cos \rho_{k}-\frac{\rho_{2 k}}{\rho_{1 k}}\right)^{2}}{\left(\cos \varphi_{k}-\frac{\rho_{1 k}}{\rho_{2 k}}\right)^{2}+\left(\cos \varphi_{k}-\frac{\rho_{2 k}}{\rho_{1 k}}\right)^{2}} m_{b j}^{2}\right]
\end{aligned}
$$

We can present a simplified expression for the error in chord length, derived by Professor I. D. Zhongolovich [20], provided that the error in the chord direction, obtained from a considerable number of observations, is negligible, compared to the errors in measuring the differences and directions to the AES in the given figure.

$$
m_{D}^{2}=\left(\cos ^{2} \varphi_{i}+\cos ^{2} \varphi_{j}\right) m_{p}^{2}+2 h^{2} m_{p}^{2} .
$$

Lambeck [58] presents the following expression for the error $/ 156$ in chord length (in the notations of Figure 15)

$$
\frac{m_{1}^{2}}{D^{2}}=\frac{\left(A C-B^{2}\right)}{(A-C-C-2 B)} m_{3}^{2}
$$

where

$$
\begin{aligned}
& A=1+\frac{\operatorname{ctg}^{2} \beta_{k}}{\sin ^{2} \beta_{i}}+\frac{\sin ^{2} \beta_{i}}{\sin ^{4} \beta_{j} \sin ^{2} \beta_{k}}, \\
& B=-\operatorname{ctg}^{2} \beta_{k}+\frac{\sin ^{4} \beta_{i}+\sin ^{4} \beta_{i}}{\sin ^{3} \beta_{i} \sin ^{3} \beta_{j}}, \\
& C=1+\frac{\operatorname{ctg}^{2} \beta_{k}}{\sin ^{2} \beta_{i}}+\frac{\sin ^{2} \beta_{i}}{\sin ^{4} \beta_{i} \sin ^{2} \beta_{k}} .
\end{aligned}
$$

If the differences in distances from both points - the chord ends - are measured by the differences in distances from the point to two AES positions in the figure for calculating the chord length, the error in chord length will be calculated by the expression 


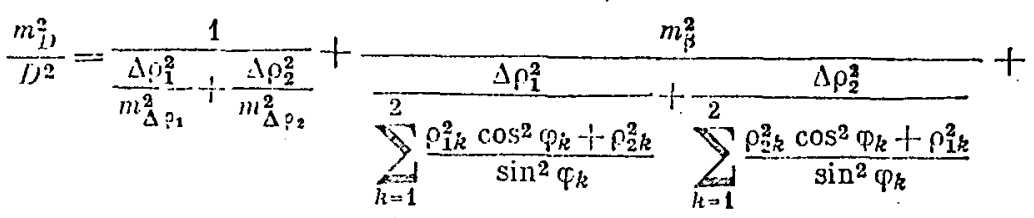

$$
\begin{aligned}
& +\frac{\Delta_{\rho_{1}^{2}}}{\sum_{k=1}^{2}\left(\frac{\rho_{1 k} \cos \varphi_{k}-\rho_{3 k}}{\sin \varphi_{k}}\right)^{2}}+\frac{\Delta \rho_{2}^{2}}{\sum_{k=1}^{2}\left(\frac{\rho_{3 k} \cos \varphi_{k}-\rho_{1 k}}{\sin \varphi_{k}}\right)^{2}}
\end{aligned}
$$

When calculating the length of the chord from several such figures or elementary figures, its error may be calculated according to Expression (33.2).

Error in Three-Dimensional Angular Intersection

When calculating the position of the point, the formula for the error in the vertex of three-dimensional angular intersection $n$ of the directions will have the form

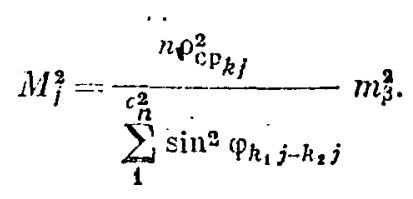

A similar formula may be used to calculate the position of the point by the directions $n$ of the chords with approximate equality of $D_{1 j}$.

If the point is calculated at the intersection of equally precise chord directions, located uniformly around the point and having an approximately identical length, then

$$
H_{j}^{2}=\frac{n \Gamma_{s}^{3} p}{\sum_{c=1}^{n-1}(n-c) \sin ^{2} \frac{2 c: \tau}{n}} m_{B}^{3} .
$$


If the error in the vertex of the elementary figure is represented by Expression (30.13) when $n$ ' directions and $n$ " planes are measured to estimate the accuracy of the figure, we may use the approximate formula

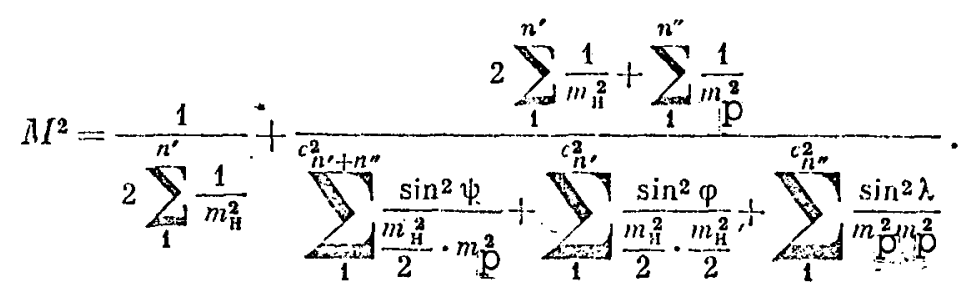

With equally precise measurements

$$
M^{2}=\frac{m_{\mathrm{R}}^{2}}{2 n^{\prime}}+\frac{\left(2 n^{\prime} m\right.}{\left.2 m_{\mathrm{M}}^{2} m_{\mathrm{p}}^{2} \sum \sin ^{2} \psi+4 m_{\mathrm{H}}^{4} m_{\mathrm{Q}}^{2}\right) m_{\mathrm{R}}^{\mathrm{a}} m_{\mathrm{p}}^{\mathrm{a}} \sin ^{2} \varphi+m_{\mathrm{p}}^{4} \sum \sin ^{2} \lambda}
$$

For all forms of linear-angular intersection

$$
M^{2}=\frac{m_{\mathrm{H}}^{2}}{2 n^{\prime}}+\frac{n^{n} \frac{m_{\mathrm{H}}^{\mathbf{2}}}{2}+-n^{\prime} m_{\ddagger}^{2}}{\sum_{i}^{c_{n}^{2}+n^{n}} \cos ^{2} \varphi_{\mathrm{H}} ?} .
$$

When calculating the position of the point by the polar method, we have

$$
M^{2}=\frac{m_{\mathrm{H}}^{2}}{n^{\prime}}+\frac{m_{\rho}^{2}}{n^{n}}
$$

Finally, for three-dimensional linear intersection in space, we will have 


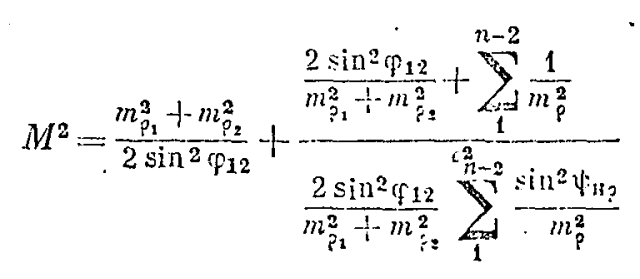

and with equally precise measurements

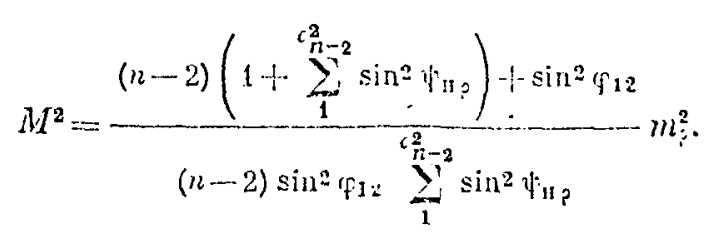

Error in the Vertex of Intersection of the Planes

Because of the complexity of the joint geometric relationships of the intersecting planes at $n>3$, we can recommend that analysis of accuracy of multiple intersection of planes or multiple linear intersection be carried out by using the expression for the error in the arithmetic mean of the results of several elementary figures, i.e.,

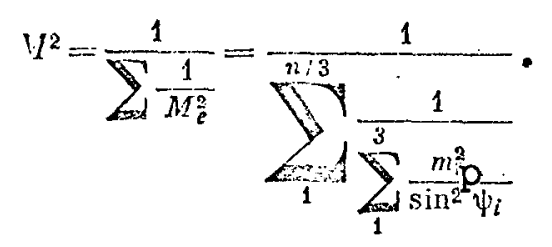

34. A Priori Estimation of the Accuracy of Coordinate Transfer in a Space Triangulation Series

Let us call a set of points, related by synchronous observations of AES positions such that we can sequentially calculate the positions of the entire set of points - using a minimum of one or two points at the beginning of the series as the starting points - a space triangulation series. 
The error in the position of the point of a space triangulation series may be represented in the form of two terms, the first of which contains the effect of measurement errors, to calculate the given point, and the second term of which contains the effect of errors in the position of points, which are the starting points for calculating this point. The error in the position of the $n$th point of the series may then be calculated by the recursion relation

$$
M_{j}^{2}=M_{0}^{2}+K^{2}\left(M_{j 1}^{2}+M_{j 2}^{2}\right)
$$

where $M_{0}$ is the error in the position of the point, caused by observation errors in the given figure, and $K$ is the coefficient of the effect of errors in the position of points $1,2, \ldots,(n-1)$ which are the starting points for calculation of the given point.

The nature of accumulation of the effect of measurement errors on the error in the position of the point of the series depends on the sequence of calculating the points.

Space triangulation series for three groups may be calculated as a function of this.

We include in the first group a serles of sequential figures when the starting point for each new point of the series is the one preceding (Figure 51).

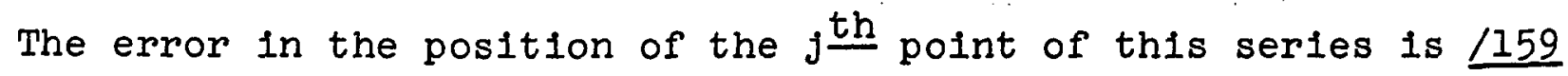
calculated by the formula

$$
M_{i}^{2}=M_{0}^{2} \sum_{j=1}^{i} K^{2(j-1)} .
$$




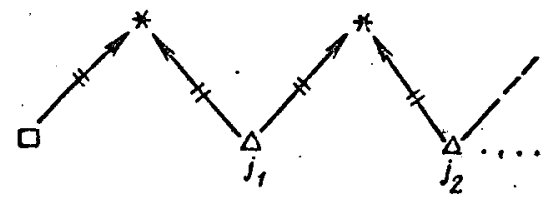

Figure 51.

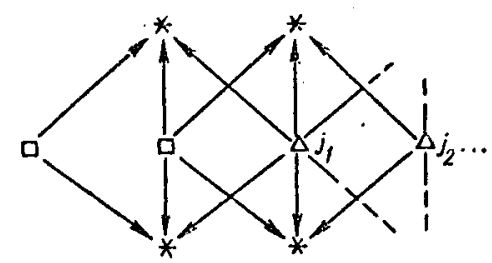

Figure 52.

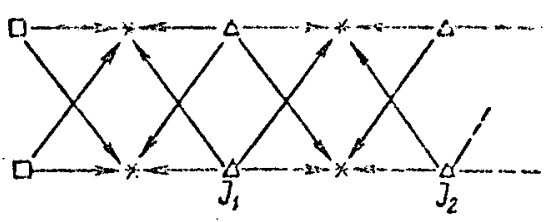

Figure 53.

We relate the series of sequential elementary figures to the second group, when each new point is calculated from the two preceding points (Figure 52).

The error in the position of the $j$ th point of the given series may be calculated by the formula

$$
M_{j}^{2}=M_{0}^{2} \sum_{j=1}^{j} a_{j} K^{2(j-1)}
$$

where the coefficients $a_{g}$ are selected from the table, similar to Pasqual's triangle:

\begin{tabular}{|c|c|}
\hline$j=1$ & 1 \\
\hline 2 & 11 \\
\hline 3 & $\begin{array}{lll}1 & 2 & 1\end{array}$ \\
\hline 4 & $\begin{array}{llll}1 & 2 & 3 & 1\end{array}$ \\
\hline 5 & 12441 \\
\hline 6 & $\begin{array}{llllll}1 & 2 & 4 & 7 & 5 & 1\end{array}$ \\
\hline 7 & $=1248116$ \\
\hline
\end{tabular}

Here each element is equal to the sum of two elements: one of them is located above the calculated element, and the second is located to the left between them. For example, in the last row, $11=4+7$.

Finally, we relate a series of sequential pairs of elementary figures in which the initial points for each new pair of points is the preceding pair of points (Figure 53) to the third group. 
The error in the position of points of this series may be calculated by the formula

$$
M_{J}^{2}=:=M_{0}^{3} \sum_{J=1}^{J} K^{2(J-1)}
$$

similar to Formula (34.2), if $\mathrm{J}$ is not the number of the point, but the number of the pair of points.

35. Errors in the Position of Points in Continuous

Space Triangulation Networks

The formulas for a priori estimation of accuracy, obtained above, are valid for individual isolated space triangulation figures. Moreover, an a priori estimation of the accuracy of continuous space triangulation networks is of interest, because in many existing designs, both foreign and Soviet [18], the development of space triangulation is in the form of a continuous network, distributed over the entire surface of the globe or a considerable part of it. It is clear that estimates of the errors for individual figures may not be simply generalized to systems of such figures of considerable length.

As we know, the accuracy of the elements of equated geodetic networks is established with the aid of welght coefficients, i.e., by the elements of the inversion matrix of a system of normal equations. Moreover, it is unnecessary to calculate the weight coefficients with the total accuracy possible for purposes of a priori estimation of accuracy. In many cases, it is sufficient to obtain the numerical characteristics with an accuracy of 20 - 30\%* in order to calculate the characteristics of error distribution in the networks.

*This is equivalent to the requirement that the error in the analysis does not exceed $1 / 5$ or $1 / 3$ of the total error. 
Approximate values of the elements of the inversion matrix may be obtained by a method known in the literature as the perturbation method. It is as follows.

It is simpler to invert diagonal matrices. Let us give the matrix of coefficients of normal equations in the form of the sum of two matrices

$$
B=P \div \varepsilon C,
$$

where $P$ is a diagonal matrix, comprised of the quadratic coefficlents of normal equations; and $\varepsilon$ is some factor, different from zero and subject to the condition $\varepsilon \leq l$.

Thus, for matrices $P$ and $C$, we will have

$$
\begin{aligned}
P & =\left[\begin{array}{cccc}
b_{11} & 0 & \ldots & 0 \\
0 & b_{22} & \ldots & 0 \\
\therefore & \ldots & \ldots & \ldots \\
0 & 0 & \ldots & b_{k k}
\end{array}\right] \\
C & =\left[\begin{array}{cccc}
0 & b_{12} & \ldots & b_{1 k} \\
b_{21} & 0 & \ldots & b_{2 k} \\
\ldots & \ldots & \ldots & \ldots \\
b_{k 1} & b_{k 2} & \ldots & 0
\end{array}\right] .
\end{aligned}
$$

Let us now expand the precise inversion matrix $B^{-1}$ into a power serles of

$$
B^{-i}=: Q=Q_{0}+\varepsilon Q_{1}+\varepsilon^{2} Q_{2}+\varepsilon^{3} Q_{3}+\ldots
$$

where

$$
Q_{0} \therefore P^{-1}=\left[\begin{array}{cccc}
\frac{1}{b_{11}} & 0 & \ldots & 0 \\
0 & \frac{1}{b_{22}} & \ldots & 0 \\
\ldots & \ldots & \ldots & \ldots \\
0 & 0 & \ldots & \frac{1}{b_{k k}}
\end{array}\right]
$$


If we multiply the matrices, calculated by Expressions (35.1) and (35.3), and take the fact into account that $B^{-1} B=E$, we will have

$$
\left(Q_{0}+\varepsilon Q_{1}+\varepsilon^{2} Q_{2}+\varepsilon^{3} Q_{3}+\ldots\right)(P+\varepsilon C)=B^{-1} B=E
$$

Performing the multiplication, we obtain

$$
Q_{0} P+\varepsilon Q_{1} P+\varepsilon^{2} Q_{2} P+\ldots \ldots+\varepsilon Q_{0} C+\varepsilon^{2} Q_{1} C+\ldots=E
$$

Matrix $Q$ is a matrix, inverse to $P$; therefore, $Q_{0} P=E$, and Expression (35.6) assumes the form

$$
\begin{array}{r}
\varepsilon Q_{1} P+\varepsilon^{2} Q_{2} P+\varepsilon^{3} Q_{3} P+\ldots+\varepsilon Q_{0} C+ \\
+\varepsilon^{2} Q_{1} C+\varepsilon^{3} Q_{2} C+\ldots=0
\end{array}
$$

or

$$
\varepsilon\left(Q_{1} P+Q_{0} C\right)+\varepsilon^{2}\left(Q_{2} P+Q_{1} C\right)+\varepsilon^{3}\left(Q_{3} P+Q_{2} C\right)+\ldots=-0
$$

By definition $\varepsilon \neq 0$, and the expressions in the parentheses are consequently equal to zero. Thus we obtain the system of equations

$$
\begin{aligned}
& Q_{1} P=-Q_{0} C, \\
& Q_{2} P=-Q_{1} C, \\
& Q_{3} P=-Q_{2} C .
\end{aligned}
$$

Let us multiply the matrix equations (35.9) from the right by $Q_{0}$, then, taking the fact into account that $P_{0}=E$, we will have 


$$
\begin{aligned}
& Q_{1}=-Q_{0} C Q_{0}, \\
& Q_{2}=-Q_{1} C Q_{0}, \\
& Q_{3}=-Q_{2} C Q_{0},
\end{aligned}
$$

1.e., the terms of Series (35.3) are described by the following recursion formula:

$$
Q_{t+1}=-Q_{i} C Q_{0}
$$

Assuming for further calculations that $\varepsilon=1$, we obtain the 1162 following group of formulas for calculating the elements of the inversion matrix:

$$
\begin{gathered}
B=P+C, \\
B^{-1}=Q, \\
p^{-1}=Q_{0}, \\
Q_{1}=-Q_{0} C Q_{0} \ldots Q_{t+1}=-Q_{l} C Q_{0}, \\
Q=Q_{0}+Q_{1}+Q_{2}+\cdots
\end{gathered}
$$

Let us now turn to a calculation of the approximate values of the weight coefficients by Formulas (35.12).

Matrix $Q_{0}$ is inverse to diagonal matrix $P$

$$
P^{-1}=Q_{0}=\left[\begin{array}{cccc}
\frac{1}{b_{11}} & 0 & \ldots & 0 \\
0 & \frac{1}{b_{22}} & \ldots & 0 \\
\ldots & \ldots & \ldots & \ldots \\
0 & 0 & \ldots & \frac{1}{b_{k k}}
\end{array}\right]=\left[\begin{array}{llll}
q_{11}^{0} & 0 & \ldots & 0 \\
0 & q_{22}^{0} & \ldots & 0 \\
\ldots & \ldots & \ldots & \ldots \\
0 & 0 & \ldots & q_{k k}^{0}
\end{array}\right] .
$$


Let us find matrix $Q_{1}$

$$
\begin{aligned}
Q_{1}= & -Q_{0} C Q_{0}=-\left[\begin{array}{cccc}
q_{11}^{0} & 0 & \ldots & 0 \\
0 & q_{22}^{0} & \ldots & 0 \\
\ldots & \ldots & \ldots & \ldots \\
0 & 0 & \ldots & q_{k k}^{0}
\end{array}\right]\left[\begin{array}{llll}
0 & b_{12} & \ldots & b_{1 k} \\
b_{21} & 0 & \ldots & b_{2 k} \\
\ldots & \ldots & \ldots & \ldots \\
b_{k 1} & b_{k 2} & \ldots & b_{k k}
\end{array}\right] \times \\
& \times\left[\begin{array}{ccccc}
q_{11}^{0} & 0 & \ldots & 0 \\
0 & q_{22}^{0} & \ldots & 0 \\
\ldots & \ldots & \ldots & \ldots \\
0 & 0 & \ldots & q_{k k}^{0}
\end{array}\right]=-\left[\begin{array}{cccc}
0 & q_{12}^{\prime} & \ldots & q_{1 k}^{\prime} \\
q_{21}^{\prime} & 0 & \ldots & q_{2 k}^{\prime} \\
\ldots & \ldots & \ldots & \ldots \\
q_{k 1}^{\prime} & q_{k 2}^{o} & \ldots & 0
\end{array}\right] .
\end{aligned}
$$

Similarly, we obtain matrix $Q_{2}$

$$
\begin{aligned}
& Q_{2}=-Q_{1} C Q_{0}=\left[\begin{array}{cccc}
0 & q_{12}^{\prime} & \ldots & q_{1 k}^{\prime} \\
q_{21}^{\prime} & 0 & \ldots & q_{2 k}^{\prime} \\
\ldots & \ldots & \ldots & \ldots \\
g_{k 1}^{\prime} & g_{k 2}^{\prime} & \ldots & 0
\end{array}\right]\left[\begin{array}{cccc}
0 & b_{12} & \ldots & b_{1 k} \\
b_{21} & 0 & \ldots & b_{2 k} \\
\ldots & \ldots & \ldots & \ldots \\
b_{k 1} & b_{k 2} & \ldots & 0
\end{array}\right] \times \\
& \times\left[\begin{array}{llll}
q_{11}^{0} & 0 & \ldots & 0 \\
0 & q_{22}^{0} & \ldots & 0 \\
\ldots & \ldots & \ldots & \ldots \\
0 & 0 & \ldots & q_{k k}^{n}
\end{array}\right]=\left[\begin{array}{llll}
q_{11}^{n} & q_{12}^{*} & \ldots & q_{1 k}^{*} \\
q_{21}^{\prime \prime} & q_{22}^{*} & \ldots & q_{2 k}^{*} \\
\ldots & \ldots & \ldots & \ldots \\
q_{k 1}^{n} & q_{k 2}^{*} & \ldots & q_{k k}^{*}
\end{array}\right]
\end{aligned}
$$

Matrix $Q_{3}$ comprises the following approximation

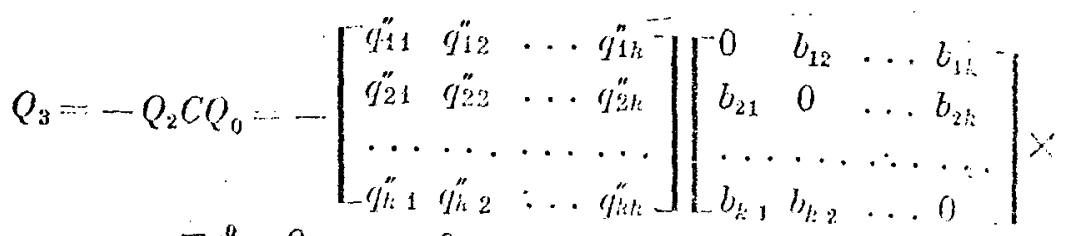

$$
\begin{aligned}
& \times\left[\begin{array}{llll}
q_{11}^{0} & 0 & \ldots & 0 \\
0 & q_{22}^{0} & \ldots & 0 \\
\cdots & \ldots & \ldots & \ldots \\
0 & 0 & \ldots & q_{h h}^{0}
\end{array}\right]=\left[\begin{array}{llll}
q_{11}^{\prime \prime} & q_{12}^{\prime \prime} & \ldots & q_{11}^{\prime \prime \prime} \\
q_{21}^{\prime \prime \prime} & q_{22}^{\prime \prime} & \ldots & q_{2 i}^{\prime \prime} \\
\ldots & \ldots & \ldots & \ldots \\
q_{k 1}^{\prime \prime \prime} & q_{h 2}^{\prime \prime \prime} & \ldots & q_{h k}^{\prime \prime}
\end{array}\right] .
\end{aligned}
$$

etc. 


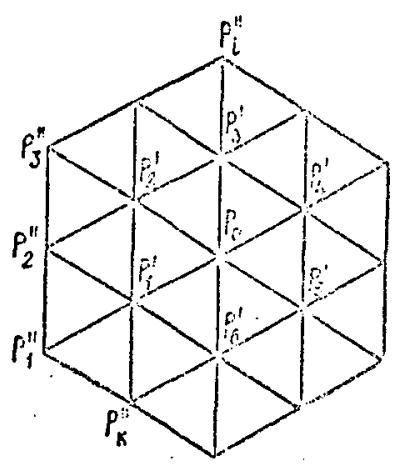

Figure 54 .

Having carried out the operation of multiplication of the matrices according to $(35.14)-(35.16)$, we obtain the diagonal elements of matrices $Q_{0}, Q_{1}, Q_{2}$, and $Q_{3}$, expressed by the coefficients of normal equations

$$
\begin{aligned}
& q_{i i}^{0}=\frac{1}{\dot{b}_{i i}}, \\
& q_{i i}^{\prime}=0 \text {, } \\
& q_{i i}^{\prime \prime \prime}=\frac{1}{b_{i i}} \sum \frac{b_{i i}^{2}}{b_{i l i} b_{j i}}, \\
& q_{i i}^{\prime \prime}=-\frac{1}{b_{i i}} \sum_{i j} \frac{b_{i j}}{b_{p p}} \sum_{i i} \frac{b_{i j} b_{j p}}{b_{i t} b_{i j}} .
\end{aligned}
$$

The arbitrary diagonal element of the precise inversion matrix $Q$ is expressed by the sum

$$
q_{t i}=q_{i i}^{0}+q_{i i}^{\prime}+q_{i i}^{\prime \prime}+\ldots
$$

The problem of the feasible number of approximations for Series (35.18) was closely related to the problem of localizing the measurement errors in continuous geodetic networks.

A continuous geodetic network, formed by three-dimensional lines, is depicted in Figure 54. The central point of this network is $P_{0}$. Measurement errors, made at adjacent points, will affect the position of point $\mathrm{P}_{0}$. It is natural that the first series of points $\left.\left(P_{1}^{\prime}, P^{\prime}, \ldots, P^{\prime}\right)_{6}\right)$ will have the strongest effect, and the second series $\left(P{ }_{1}, P^{\prime \prime}, \ldots, P^{\prime \prime}{ }_{k}\right)$ has a less noticeable effect. Further, this effect will be more and more weakened. From the theoretical point of view, all points of the network, no matter how far it extends, will affect the position of point $P_{0}$. However, beginning at some series of points, this 
effect will barely increase. We manage to obtain numerical estimates of the effect of remote points. The error in the position of a given point of the network is represented in the form

$$
M_{t}=\mu \sqrt{q_{\lambda_{i}}+q_{y_{i}}+q_{z_{i}}}=\mu r \sqrt{\frac{1}{p_{i}}} .
$$

Variation of the value $\sqrt{1 / p_{i}}$ as a function of the number of 1164 approximations is shown below.

\begin{tabular}{lllllllllll}
$\begin{array}{c}\text { Number of } \\
\text { approximations }\end{array}$ & 1 & 2 & 3 & 4 & 5 & 6 & 7 & 8 & $\ldots$ & $\infty$ \\
\hline$\sqrt{1 / p_{i}}$ & 0.71 & 0.76 & 0.81 & 0.84 & 0.87 & 0.89 & 0.91 & 0.92 & $\ldots$ & 1.00
\end{tabular}

Moreover, we established that the approximation number essentially corresponds to the number of the subsequent series of points whose effect is taken into account in the given approximation. Thus, matrix elements depend only on the quadratic coefficients of the normal equations, i.e., they are calculated on the basis of the information which is obtained at a given point. The elements of matrix $Q_{2}$ are calculated by the non-quadratic coefficients of normal equations of type $b_{i j}$, which pertain only to a single normal equation. In other words, the effect of the first series of surrounding points is calculated with the aid of these coefficients. Products of non-quadratic coefficients $b_{i j}, \ldots$, $b_{j p}$, appear in the diagonal elements of matrix $Q_{3}$, $i . e$. , the effect of the second series of points is taken into account in this approximation. Thus, the effect of more and more distant points of the network is taken into account with each new approximation.

If from a number of approximations we select a region where the error due to the effect of measurement errors, made in 
succeeding series of surrounding points, is less than $1 / 5$ of the total error in the reduced dependence $\sqrt{1 / p_{1}}$, it turns out that the effect of the fourth series of points may be disregarded. Thus, the entire section of the network, over whose length there is essentially complete attenuation of the effect of measurement errors on the position of the given point, comprises three to four serles of points on each side.* Hence, we may conclude that it is sufficient to limit ourselves to three terms in Series (35.18). Then

$$
q_{i l}=\frac{1}{b_{i l}}\left(1+\sum \frac{b_{i i}^{2}}{b_{i i} b_{j i}}\right)
$$

Formula (35.20) expresses the approximate elements of the inversion matrix by the coefficients of normal equations. In turn, the coefficients of normal equations will depend on the shape of the network and on the form of geodetic information which is used to construct the given network.

Let the topocentric coordinates $\delta_{i}$ and $\gamma_{i}$ and distances to the AES $\rho_{i}$ be measured in the network.

For the calculation, we assume that in the expression for the error of the vector connecting the ground point to the AES position,

$$
M_{p}^{2}=\left(m_{\delta}^{2}+m_{\gamma}^{2} \cos ^{2} \delta\right) \rho^{2}+m_{p}^{2}
$$

all three components are equal to each other, i.e.,

$$
m_{\delta}^{2}=m_{\curlyvee}^{2} \cos ^{2} \delta=\frac{m_{\varphi}^{2}}{\rho^{2}} .
$$

* It is appropriate to recall that the same result was obtained by $K$. L. Provorov with the aid of other means for twodimensional networks. 
Then, assuming that the mean square error of unit weight $\mu=\mathrm{m}_{\delta}$, we obtain the following expressions for the weights:

$$
p_{0}=1 ; \quad p_{\gamma}=\cos ^{2} \delta ; \quad p_{0}=\frac{1}{p^{2}}
$$

Under these conditions, the matrix of coefficients of normal equations $B_{\gamma}, \delta, \rho$ has the form

$$
\begin{aligned}
& B_{\gamma, \delta, ?}= \\
& =\left[\begin{array}{cccccccccc}
{\left[\frac{1}{\rho^{2}}\right]} & 0 & 0 & -\frac{1}{\rho_{1}^{2}} & \ldots & \ldots & \ldots & -\frac{1}{\rho_{N}^{2}} & 0 & 0 \\
0 & {\left[\frac{1}{\rho^{2}}\right]} & 0 & 0 & -\frac{1}{\rho_{1}^{2}} & \ldots & \ldots & 0 & -\frac{1}{\rho_{N}^{2}} & 0 \\
0 & 0 & {\left[\frac{1}{\rho^{2}}\right]} & 0 & 0 & -\frac{1}{\rho_{1}^{2}} & \ldots & 0 & 0 & -\frac{1}{\rho_{N}^{2}} \\
\cdots & \cdots & \cdots & \ldots & \ldots & \cdots & \ldots & \ldots & \ldots & \cdots
\end{array}\right] .
\end{aligned}
$$

The structure of Matrix (35.24) leads to the conclusion that, if the spherical coordinates of the $\operatorname{AES} \gamma, \delta$, and $\rho$ in the space geodetic network are measured with weights which are subject to the relations (35.23), the system of normal equations separates into three independent parts: corrections of the $x$-coordinates are calculated from the solution of the first, those of the $y-$ coordinates - by the second, and finally, those of the zcoordinates - from solution of the third.

The expressions for elements of matrix $B_{\gamma}, \delta, \rho$ of (35.24) may be simplified if the distances between the points of the network and the AES positions are assumed to be identical, 1.e., if it is assumed that $\rho_{1}=\rho_{2}=\rho_{k}=\rho$. Let us multiply matrix ${ }^{B}, \delta, \rho$ by the scalar quantity $\rho^{2}$, and as a result we obtain matrix $\bar{B}_{\gamma, \delta, \rho}$ of very simple form 


$$
\begin{aligned}
& \bar{B}_{\gamma, \delta, p}=\rho^{2} B_{\gamma, \delta, ?}= \\
& =\left[\begin{array}{ccccccccccccccc}
N_{1} & 0 & 0 & -1 & 0 & 0 & -1 & \ldots & \ldots & \ldots & \ldots & -1 & 0 & 0 \\
0 & N_{1} & 0 & 0 & -1 & 0 & 0 & -1 & \ldots & \ldots & \ldots & 0 & -1 & 0 \\
0 & 0 & N_{1} & 0 & 0 & -1 & 0 & 0 & -1 & \ldots & \ldots & 0 & 0 & -1 \\
-1 & 0 & 0 & N_{1} & 0 & 0 & -1 & 0 & 0 & -1 & \ldots & -1 & 0 & 0 \\
\ldots & \ldots & \ldots & \ldots & \ldots & \ldots & \ldots & \ldots & \ldots & \ldots & \ldots & \ldots & \ldots & \ldots
\end{array}\right]
\end{aligned}
$$

The diagonal elements of Matrix (35.25) are equal to the number of directions, convergent at a given point (the AES position). Each row of the matrix will contain as many ones as there are connections with the AES positions surrounding it at a given point. The remaining elements are equal to zero.

If a space geodetic network is constructed only from measured angles $\gamma$ and $\delta$, the matrix of normal equations will be

$$
\begin{aligned}
& \bar{B}_{y, 8}=\rho^{2} S_{i ;}, 8=
\end{aligned}
$$

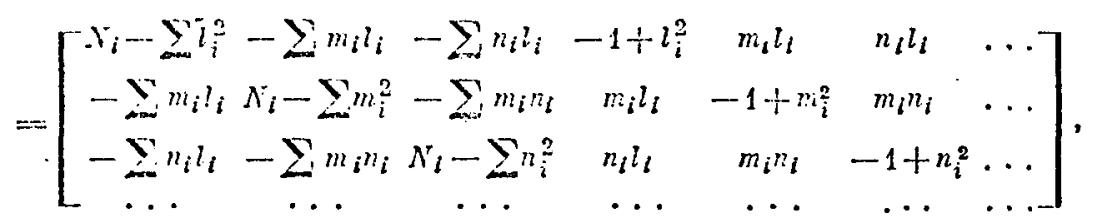

where $z_{1}, m_{1}$, and $n_{1}$ are the direction cosines of the directions to the AES; and $\mathrm{N}_{1}$ is the number of directions convergent at a given point or at the satellite.

For a network with measured distances to the AES, the matrix of normal equations has the form

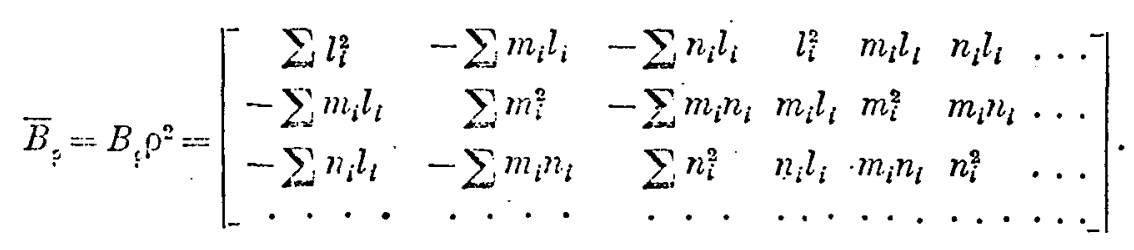


By having specific expressions of the matrix elements of normal equations, which occur in space networks with different measurements, it is easy to obtain formulas for an approximate calculation of the weight coefficients according to the general expression $(35.20)$.

Let the spherical coordinates $\gamma$ and $\delta$ and distances $\rho$, whose errors are assumed to be equal for simplicity, be measured in the network. For this case, Formula (35.20) assumes the form

$$
q_{i l}=q_{x_{i}}=q_{y_{i}}=q_{z_{i}}=\frac{\rho^{2}}{N_{i}}\left(1+\sum_{i=1}^{N_{l}} \frac{1}{N_{i} N_{i}}\right),
$$

where $N_{i}$ is the number of directions measured at a given point, and $\mathrm{N}_{\mathrm{J}}$ is the number of directions at adjacent points, connected to point $i$. If we use the average number of directions in the network. $\left(N_{i}=N_{j}\right)$, we will have

$$
q_{n}=\frac{\rho^{2}}{N}\left(1+\frac{1}{N}\right)
$$

For the error in the position of the point, we obtain

$$
M_{l}^{2}=\mu \sqrt{q_{x_{l}}+q_{y_{l}}+q_{z_{l}}}=\mu_{0} \sqrt{\frac{3}{N}\left(1+\frac{1}{N}\right)} .
$$

Let us now consider a network in which only angular values were measured - spherical coordinates $\gamma$ and $\delta$. Substitution of the specific values of the coefficients of normal equations into Formula (35.20) after slight simplifications leads to the expressions 


$$
\begin{aligned}
& q_{x_{i}}=\frac{\rho^{2}}{N_{l}-\sum l_{i}^{2}}\left[1+\frac{N_{i}-\sum l_{i}^{4}}{\left(N_{i}-\sum l_{i}^{2}\right)\left(N_{j}-\sum q^{2}\right)}\right], \\
& q_{y_{i}}=\frac{\rho^{2}}{N_{l}-\sum m_{i}^{2}}\left[1+\frac{N_{l}-\sum m_{i}^{4}}{\left(N_{i}-\sum m_{i}^{2}\right)\left(N_{j}-\sum q^{2}\right)}\right], \\
& q_{z_{i}}=\frac{\rho^{2}}{N_{i}-\sum n_{i}^{2}}\left[1+\frac{N_{l}-\sum n_{i}^{4}}{\left(N_{i}-\sum n_{i}^{2}\right)\left(N_{j}-\sum q^{2}\right)}\right],
\end{aligned}
$$

in which $\Sigma q^{2}$ is the mean value of the sum of squares of the direction cosines of the measured directions with respect to some coordinate axis. The error in the position of the point in such a network will be

$$
M_{i}=\mu \rho \sqrt{\left(\frac{1}{N_{l}-\sum_{i} l_{i}^{2}}+\frac{1}{N_{i}-\sum m_{i}^{2}}+\frac{1}{N_{i}-\sum n_{i}^{2}}\right)\left(1+\frac{N_{l}-\sum q^{2}}{\left(N_{l}-\sum q_{i}^{2}\right)^{2}}\right)} .
$$

In a network constructed from the measured distances to the AES, the formulas for the approximate values of the weight coefficients have the form

$$
\begin{aligned}
& q_{x_{i}}=\frac{\rho^{2}}{\sum l_{i}^{2}}\left[1+\frac{2 \sum l_{i}^{2}-\sum l_{i}^{4}}{\sum l_{i}^{2} \sum q^{2}}\right], \\
& q_{y_{i}}=\frac{\rho^{2}}{\sum m_{i}^{2}}\left[1+-\frac{2 \sum m_{i}^{2}-\sum m_{i}^{4}}{\sum m_{i}^{2} \sum q^{2}}\right], \\
& q_{z_{i}}=\frac{\rho^{2}}{\sum n_{i}^{2}}\left[1-\frac{2 \sum n_{i}^{2}-\sum n_{i}^{4}}{\sum n_{i}^{3} \sum q^{2}}\right] .
\end{aligned}
$$

Finally, for a combined network, in which some of the sides are measured in addition to the spherical coordinates $\gamma$ and $\delta$, we w111 have

$$
q_{x_{i}}=\frac{\rho^{2}}{N_{i}-\sum_{1}^{N-t} l_{i}^{2}}\left[1+\frac{N_{i}-\sum_{1}^{N-t} l_{i}^{4}}{\left(N_{i}^{2}-\sum_{i}^{N-t} l_{i}^{2}\right)\left(N_{i}-\sum_{1}^{N-1} q^{2}\right)}\right] \text {, }
$$




$$
\begin{aligned}
& q_{y_{i}}=\frac{p^{2}}{x_{i}-\sum_{1}^{N-t} m_{i}^{2}}\left[1+\frac{N_{i}-\sum_{1}^{N-t} m_{i}^{4}}{\left(N_{i}-\sum_{i}^{N-t} m_{i}^{2}\right)\left(N_{i}-\sum_{1}^{N-t} q^{2}\right)}\right], \\
& q_{z_{i}}=\frac{p^{2}}{N_{l}-\sum_{1}^{N-t} u_{i}^{2}}\left[1+\frac{N_{i}-\sum_{1}^{N-t} n_{i}^{4}}{\left(N_{i}-\sum_{1}^{N-t} n_{i}^{2}\right)\left(N_{i}-\sum_{1}^{N-t} q^{2}\right)}\right],
\end{aligned}
$$

where $t$ is the number of sides of a network measured at a given point.

Formulas (35.34) are a generalization of Expressions (35.28) and (35.31), since at $t=N$ (when the distances are measured for all directions), they will transform to Formulas (35.28), and at $t=0$ (i.e., only angular values were measured at the point), they will transform to Formulas (35.31). 


\section{CHAPTER 7}

DATA ON DESIGN OF SPACE TRIANGULATION

\section{Fundamentals of Space Triangulation Design}

Design of geodetic networks is included in a determination of the location of points on the Earth's surface provided that the specific general requirements, valid for any constructions, are followed. Their main requirements are:

- the density of the points should correspond to the purpose of the network and to the purposes of its future use;

- the mutual distribution of points (the shape of the network) should provide for calculation of the elements of the network with the required accuracy;

- construction of the network should be carried out with minimum labor and material expenditures.

Geodetic networks, constructed with the aid of AES observations - space triangulation networks - have a number of characteristics. Continuous space triangulation networks should be considered primarily as a set of ground points and fixed instantaneous positions of an AES in orbit (i.e., the points of space observed from several ground points simultaneously). The number of measured values belonging to the ground points will usually differ from the number of measured values, comprising a certain 
synchronous group, i.e., belonging to a given AES position. The number of the latter will usually be limited by the number of ground points from which the given position of the satellite may be physically observed. The number of such measurements, convergent at some ground point, is theoretically unlimited.

Figuratively speaking, a space triangulation network may be regarded as a two-story structure. Construction of the "second story" begins first - the AES positions in circumterrestrial space are calculated. Each point of this story is relatively weak due to the small number of measurements. However, a set of such points makes it possible to obtain the coordinates of ground points (i.e., the points of the first "story") with comparatively high accuracy. Hence, it is clear that space triangulation design may not be reduced to selecting the location of ground points alone. It is just as important to provide optimum distribution of the ground points and observed AES positions with respect to each other. This means that space triangulation design includes selecting the orbital parameters of the AES and the designation of the ephemerides for observations.

Another characteristic of space triangulation is the absence of direct observations between ground points. The connections between them are accomplished by the satellite positions. Moreover, due to the considerable separation between ground points, the observation conditions at them may differ sharply. It may happen that the optimum accuracy of a space triangulation figure cannot be realized due to disturbances in the conditions of AES visibility. The concept of "visibility between points" in space triangulation is considerably more complex than in ordinary geodetic networks. Actually, at all points from which a given satellite is being observed, the following conditions should be adhered to: 
- the elevation of the satellite above the horizon may not be below a specific limit;*

- there is line-of-sight (geometric) visibility between the observation point and the AES;

- the mutual location of the Sun and Earth, the satellite and the observation point make it possible to photograph the satellite on the background of stars.

Thus, space triangulation must include calculations of the optimum observation conditions and their comparison with real observation conditions at the points.

The effect of errors in such a network and in its individual figures provides the scientific basis for compiling space triangulation designs.

The nature of the distribution and the effects of errors determine the most general requirements which should be fulfilled in construction of space triangulation networks and figures. This is related to the measurements and their accuracy, the geometric characteristics of the network, and the distribution and accuracy of starting points.

Specific design will always be related to the selection of the optimum variant of the network structure under certain limiting conditions. These may be: physical and geographic conditions, the given value of some elements of the network, the necessity of using AES already launched, etc.

*This limit is determined by the possibility of calculating refraction errors. 
The requirements on the optimum conditions of network structure may be defined by obtaining the required accuracy or by obtaining some accuracy within a given observation period.

The problem of space triangulation design may be solved within broad or narrow limits.

The more common case is creation of a design on the basis $\underline{1171}$ of the purpose of the overall goal. In this case, the optimum data from the point of view of accuracy within the established period of observations should be determined: mutual distribution and separation of observation points, the number of AES and their parameters (mainly, altitude, declination, and launch time), and AES observation zones from each of the points.

When the position of the points is given, the optimum orbital parameters of the AES and the observation zones are calculated. On the other hand, if the orbital parameters of the AES are given, the optimum distances between points, their location, and then the observation zones are selected.

In the latter case, when both the position of the points and the orbit of the AES are given, the procedure reduces to establishing the boundaries of the optimum zones and the number of observations in these zones. As a result, the locations of the ground stations and the desired orbital parameters of the AES are indicated, the a priori errors of the network elements are calculated, and the areas of the subsatelite points over which It is desirable to observe the AES are indicated in documents in numerical or graphic form. Finally, the AES observation conditions at each point should be calculated and the approximate long-term forecast of observations of the AES and its ephemerides, 
which are required for organization and planning of simultaneous observations at a group of ground stations contained in the given network, should be compiled.

The first part of the data, as in ordinary triangulation, is the essential part of the procedure. The second part, similar to the observation program at an ordinary trinagulation point, is an independent problem of calculating the AES ephemerides for observations.

In connection with the foregoing, the problems of space triangulation design are outlined in the following sequence:

- study of the overall characteristics of error effects;

- calculation of the optimum characteristics of the networks and AES orbits;

- compilation of the space triangulation design;

- calculation of the visibility conditions and ephemerides of AES observations.

In this paper, the latter problem - the principles and methods of calculating the ephemerides of AES observations for the observation points - will not be considered.

37. General Analysis of the Formulas for A Priori Estimation of the Accuracy of the Elements and Elementary

Figures of Space Triangulation

Combinations of individual figures are used to calculate the position of both single points as well as those of space triangulation series and networks. Moreover, the interrelationship between different geometric parameters and their effect on the 
accuracy of the result are simpler and more descriptive in the elementary figures. Therefore, establishment of the optimum geometric parameters of the elementary figures of space triangulation is the main problem, which precedes the correct design of space triangulation networks.

A superficial examination of the expressions for an a priori $/ 172$ estimation of the accuracy of the elements and elementary figures of space triangulation makes it possible to make some judgments about the optimum values of the geometric parameters.

Thus, to construct elementary figures containing directions measured from the observation points to the AES positions, it is desirable that these directions be measured by the shortest distances. Consequently, low-orbit AES are optimum for photographic observations; it is preferable that their routes pass through the observation point. A value of the intersection angle, close to $90^{\circ}$, is optimum for all intersections of directions and planes, and a value of the intersection angle equal to zero or $180^{\circ}$ for linear-angular intersection.

These general requirements are perceived directly from the given formulas.

However, most expressions for the errors in the elementary figures are functions of several geometric parameters, and it may not always be possible to judge the optimum value of all geometric parameters by the form of the formula. Thus, for example, the expression for the error in the direction of the chord (32.2) indicates that the error is directly proportional to the distances from the points to the AES and inversely proportional to the sine of the angle between the planes. The requirement of 
minimum distances is contradictory to some extent to the requirement that the value of the angle between the planes be close to $90^{\circ}$. Some compromise optimum condition should be sought.

If we may assume, based on the error in the position of the hyperboloid surface, that the requirements of the maximum length of the celestial chord and the differences in distances, equal to zero, are optimum, 1.e., the symmetry of Doppler observations with respect to the path, we may assume (based on the error in the length of the focal radius of the hyperboloid) on the other hand, that the maximum difference in distances, $i . e .$, the maximum asymmetry with respect to the path, is optimum. Therefore, it is difficult to make a judgment about the optimum combination of values $\Delta \rho, d$ and $l$ at first glance.

Besides the foregoing, a number of cases may be presented when establishing the optimum parameters, formally corresponding to the minimum error, is often unreal or unattainable, and is sometimes generally meaningless with respect to the problem being solved.

It is most valid to solve a system of equations of form $\partial \mathrm{M} / \partial \mathrm{v}=0$, from which we also obtain the optimum parameters, in order to find the optimum geometric parameters $v_{i}$, contained in the formulas for an a priori estimation of the accuracy of the figures. However, the expressions for the errors due to independent geometric parameters are often very cumbersome, and solution of the systems is complex. This may be accomplished only for individual elementary figures. Therefore, the optimum geometric $/ 173$ parameters, contained in the formulas for an a priori estimation of the accuracy of the elements and figures of space triangulation, are usually calculated by numerical methods. 
Calculation of the Chord Direction

The formula for the error in the chord direction (32.2) makes it possible to arrive at several conclusions immediately. First, the error will be minimum at equal distances from the points to the AES positions, i.e., the AES positions should be located symmetrically with respect to the center of the chord in a plane perpendicular to it and passing through its middle. Secondly, it follows from (32.2) that the intersection angle at an AES position, equal to $180^{\circ}$, is optimum, 1.e., observation of an AES position, located on the chord itself, is essentially absurd.

Actually, the combination of these conditions indicates the feasibility of AES observations at the minimum elevations above the horizon of the observation point.

At a given AES altitude ( $H$ ) and at a given minimum permissible value of AES elevation above the horizon of the observation point $\left(\alpha_{\min }\right)$, we can find the maximum distance from the point to the AES position by the formula

$$
\rho_{\max }=\sqrt{(R+H)^{2}-R^{2}} \overline{\cos ^{2} \alpha_{\min }}-R \sin \alpha_{\min }
$$

where $R$ is the mean radius of the Earth.

The most suitable shape of the figure from which we calculate the direction of the chord will depend on the optimum chord length. The value $D_{\text {opt }}$ is obtained from the solution of the equation

$$
\begin{gathered}
\frac{d m_{B}}{d D}=\frac{1}{4}\left(A-8 B^{2}-12 B C-5 C^{2}\right) D^{3}+. \\
+\left(5 A B+6 A C-4 B^{2} C-7 B C^{2}-4 C^{3}\right) D^{6}- \\
-3\left(A^{2}-4 A B C-6 A C^{2}+C^{4}\right) D^{1}- \\
-\left(2 A^{2} C-A B C^{2}-4_{4} A C^{3}-B C^{2}\right) D^{2}-4-A C^{2}\left(A-C^{2}\right)=0,
\end{gathered}
$$




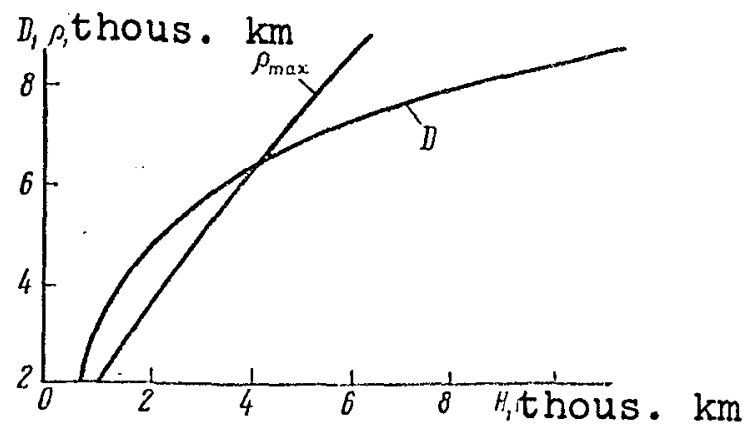

Figure 55.

where

$$
\begin{gathered}
A=4 R^{2} \rho_{\operatorname{mix}}^{2} ; \\
B==\left(R^{2}+\rho_{\max }^{2}\right) \\
C=\left(2 R I I+I^{2}-\rho_{\max }^{2}\right) .
\end{gathered}
$$

The dependence of optimum chord length on AES altitude is shown in the graph (Figure 55) for $\alpha_{\text {min }}=20^{\circ}$ (the optimum angle between the planes [ $\left.\lambda_{\text {opt }}\right]$ essentially does not vary as altitude varles and is equal to $76^{\circ}$ ). This graph may be used to select the optimum AES altitude for space triangulation with given chord lengths.

If the chord length is less than its optimum value for a given AES altitude, the most suitable shape of the figure will be determined by the distances from the points to the AES positions symmetrically located. In this case, the expression for the error in the chord direction may be represented as a function of the distances to the AES. The optimum value of the distance to the AES $\rho_{\text {opt }}$ is calculated from the solution of the equation

$$
\begin{gathered}
\frac{d m_{B}}{d \rho}=4 \rho^{8}-(3 A-4 C) \rho^{6}+ \\
+(4 A B+5 A C-32 B C- \\
\left.-20 C^{2}\right) \rho^{4}-\left(A^{2} B-12 A B C+-\right. \\
\left.+A C^{2}-12 C^{3}\right) \rho^{2}-A C(A B+ \\
\left.+C^{2}\right)=0
\end{gathered}
$$

where

$$
\begin{gathered}
A=D^{2}, \\
B=R^{2}-\left(\frac{D}{2}\right)^{2}, \\
C=2 R H+H^{2}+\frac{D^{2}}{2} .
\end{gathered}
$$



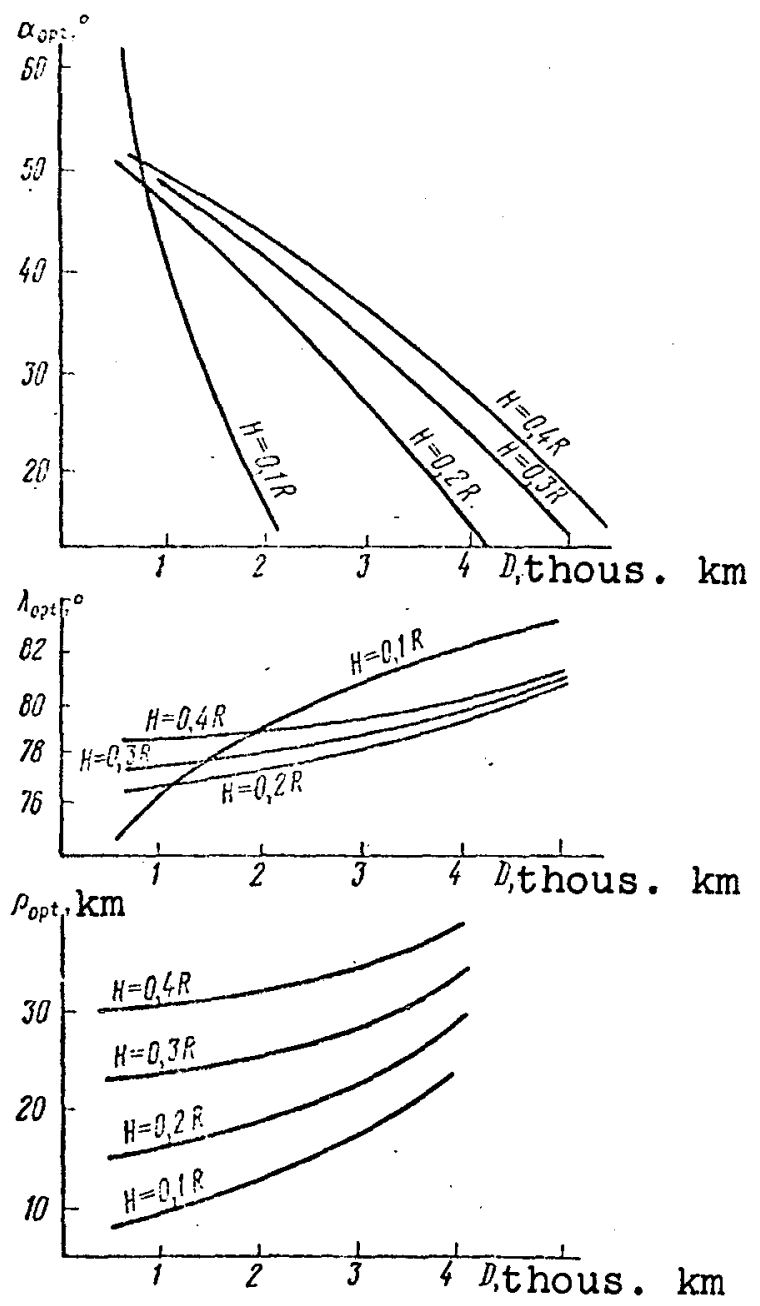

Figure 56.

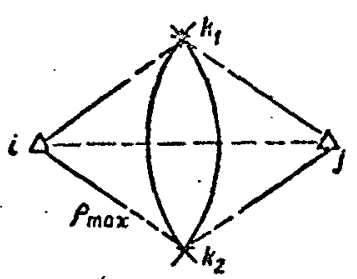

Figure 57.
The dependence of the optimum values of the distances to the AES and of the values of angles $\lambda$ and $\alpha$ on the chord length for several values of AES altitude is presented in the graph (Figure 56).

Thus, the value of the geometric parameters of the optimum figure for calculating chord direction (Figure 57), the main one of which is the ratio of AES altitude to chord length, is calculated by the graph (see Figure 55).

At a given chord length and $/ 175$ AES altitude, the optimum parameters, which determine the shape of the figure (the value of angle $\lambda_{\text {opt }}$ or of $\rho_{\text {opt }}$ ), are found from the graph (see Figure 56).

Similar graphs may be used in space triangulation from chord directions to select chord lengths, AES altitude or, if these data are established, to select the shapes of the figures for calculation of chord direction. 
Three-Dimensional Angular Intersection

'When calculating the position of the point from the directions observed from it to two known AES positions, Expression (32.3) should be used to establish the optimum parameters of the elementary figure. The distance $\rho$ may be expressed by length $d$ of the celestial chord, connecting the AES positions

$$
\rho^{2}=\frac{d^{2}}{2(1-\cos \varphi)}
$$

Having substituted (37.4) into (32.3) and having set the derivative of the expression obtained from $\varphi$ equal to zero, we obtain the cubic equation

$$
\frac{d M}{d \varphi}=\cos ^{3} \varphi+0,5 \cos ^{2} \varphi-6,5 \cos \varphi-2,5=0 .
$$

From the solution of Equation (37.5), we find three roots $(+13.382 ;-13.500$ and -0.382$)$. The root at which $\varphi_{\text {opt }}=112.5^{\circ}$ corresponds to the problem. This value of the angle also determines the optimum shape of three-dimensional angular intersection with provision of the minimum $\rho$ for the given AES altitude.

When calculating the position of the point from the intersection of the chord directions, by a similar discussion we obtain the most suitable value of the angle between the chord directions at a specific point.

However, with respect to the optimum shape of the intersection of chord directions, additional requirements enter in. These requirements are maximum advance of the figure in calculating the position of a single point and constant advance in series and in continuous space triangulation networks from the chord directions. 
The optimum shape of the figure to calculate the position of a single point should provide a minimum error in the position of the point with maximum advance of the figure, i.e., with maximum separation of the calculated point from the starting points. Having denoted the advance by $A$, we obtain the expression of this condition in the form

$$
\frac{M_{j}}{A}=\min .
$$

The shape of the figure may be characterized by the relative advance $(A / b)$, i.e., by the ratio of the absolute advance to the distance between the starting points - the base (b).

Let us consider the problem of the optimum shape of the figure for calculating the position of a single point with respect to the two main types of figures considered in section 18 , 1.e., when the point is calculated at the intersection of chord 1176 directions or by sequential three-dimensional angular intersections. In this case, we will proceed from the fact that separation of the calculated point from each of the starting points corresponds to the optimum chord length at a given AES altitude.

Expressions for the error in the position of the point in these types of figures, represented by Formulas (32.5) and (32.4), respectively, indicate that there should be an increase in the angle of intersection at the calculated point $\left(\varphi_{j}\right)$ in order to provide a minimum value of the error.

Variation of the relative advance has a different effect on changing the angle of intersection in the figures (Figure 58). With an increase of the relative advance, the angle of intersection of the chord directions decreases, whereas the zone of 


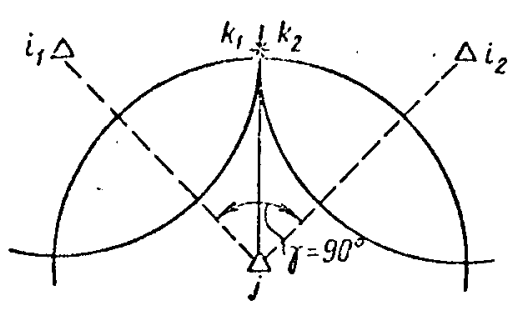

$\frac{A}{B}=0,5$

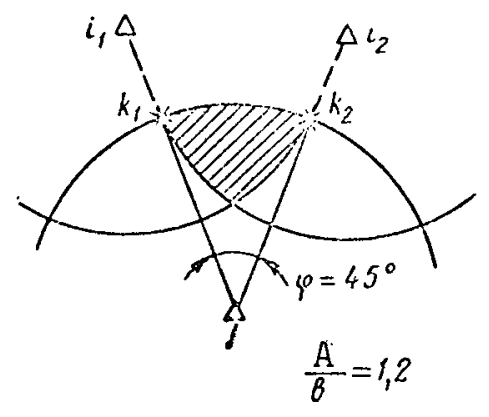

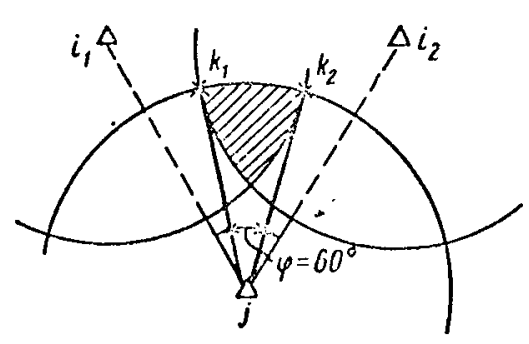

$\frac{A}{B}=0,86$

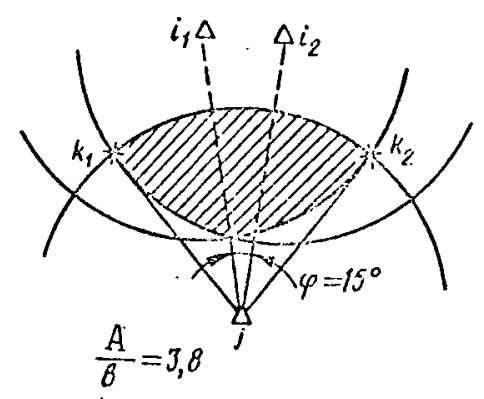

Figure 58.

simultaneous visibility of the AES from three points, and, consequently, the possibility of increasing the angle of intersection of the directions from the AES to the calculated point increases.

The nature of the variation in the accuracy of calculating the position of the point with a variation of the relative advance for these two types of figures may be judged by the graph (Figure 59), constructed for the case $H=0.25 R, \alpha_{\mathrm{min}}=20^{\circ}$, and $\mathrm{m}_{B}=1 "$.

The following may be established from the graph:

- for intersection of the chord directions, the error is minimum at $\mathrm{A} / \mathrm{b}=0.33$, which corresponds to the angle of intersection $\varphi=112^{\circ} 27^{\prime}$, but such advance of the figure is unsuitable; 


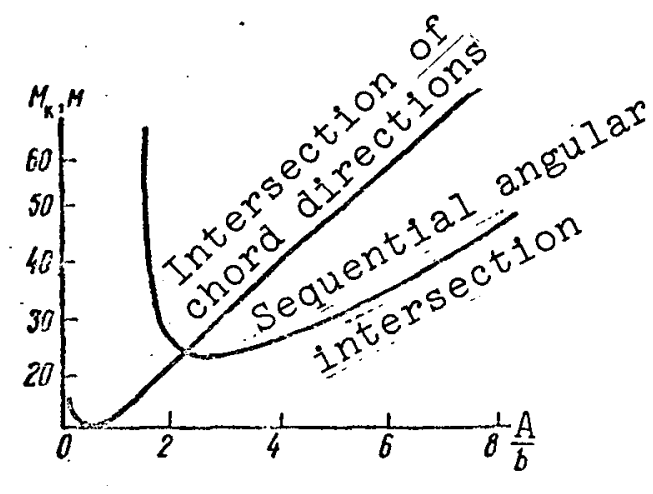

Figure 59.

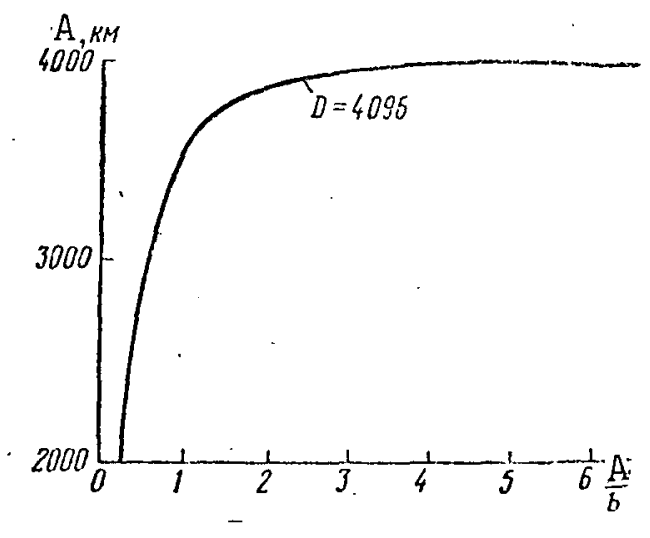

Figure 60 .

- for sequential three-dimensional angular intersections in space, the error is minimum at $\mathrm{A} / \mathrm{b}=2.36$, which corresponds to the equality of the length of the base $b$ and of the celestial chord d

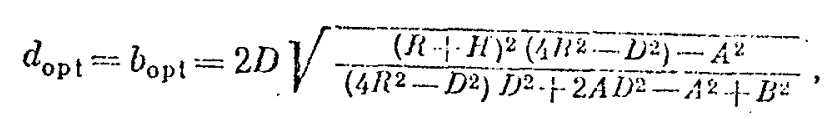

where

$$
\begin{gathered}
A=(R+H)^{2}+R^{2}-\rho^{2}, \\
B=2(R+H) R .
\end{gathered}
$$

As can be seen from the graph (see Figure 59), the optimum figure for both types of construction is one with a relative advance $A / b \approx 2.0-2.5$, and the increase of relative advance above the value of 2.5 essentially does not lead to an increase of absolute advance (Figure 60 ).

As a result of the analysis, we may conclude that the position of single points with the aid of synchronous photographic observations with a relative advance less than $\mathrm{A} / \mathrm{b}$ opt $=2.5$ should be calculated by using the three-dimensional intersection of chord directions, but in the case of a large relative advance, the sequential three-dimensional angular intersections of directions to the AES should be used. 


\section{Linear-Angular Intersection}

The formulas for the errors in the position of the point (or in the position of the AES) at linear-angular intersections $(32.12),(32.16)$, and (32.17) Indicate that the point is calculated more precisely if the angle $\varphi$ between the lines, along which the directions and distance are measured is equal to zero, i.e., from the polar intersection. The advantage of such an elementary figure, when combining photographic observations and measured distances, as can be seen from Formula (32.11), is the fact that the accuracy of calculating the position of the point does not depend on the shape of the figure. The error will be proportional to the distance to the AES and, therefore, will be minimal when observing the AES on the path of the point.

However, in the construction of space triangulation series and networks, this requirement may contradict that for maximum advance of the figure and a minimum relative error in calculating the position of the point.

Here the optimum figure will be that containing two sequential linear-angular intersections, provided that observations of the AES position are at a maximum distance from the calculated and starting points in a plane passing through them and the center of the Earth (Figure 61).

The absolute advance of such a figure will be calculated by the expression

$$
D_{\max }=\frac{2 R \rho_{\max } \cos \alpha_{\min }}{R+H}
$$

Its variation with AES altitude is shown in Figure 62. 


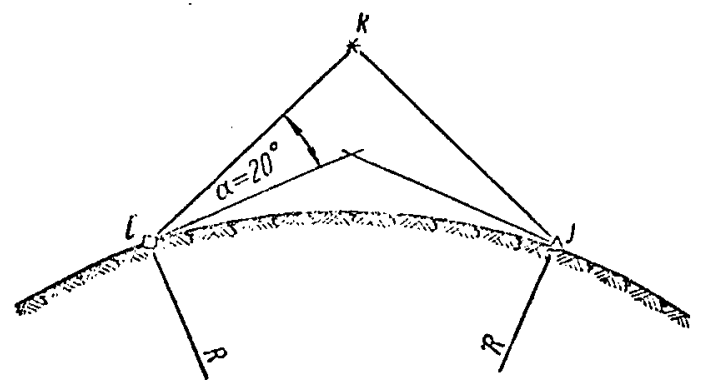

Figure 61.

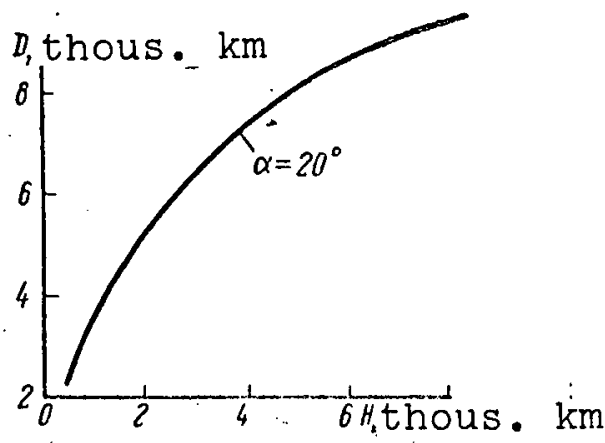

Figure 62.

When calculating the AES position from a combination of photographic observations and measurements of the differences in distances, the accuracy of intersection depends on its shape, which determines the relationship between the difference in distances and the focal radius of the corresponding hyperboloid.

It is obvious from Expressions (32.16) and (32.17) that they contain the term $\mathrm{d}^{2} \tau^{2} / \Delta \rho^{4}$, which induces contradictory requirements, on the one hand, for the maximum difference in distances $(\Delta \rho)$ and, on the other hand, for minimum values of the celestial chord (d) and separation of the point from a plane passing through the center of the chord ( 2 ). Investigation of the dependence of these values on the shape of the figure indicates that the optimum condition, satisfying the requirement $d z / \Delta \rho^{2}=\min$, will be fulfillment of observations on a trajectory passing through the zenith of a point, where one AES position should be observed at the zenith, and a second should be observed at the edge of the visibility zone. 
Calculation of the Position of a Single Point by the

Direction and the Difference in Distances

If the point is calculated from an elementary figure containing the direction and the difference in distances from the point to the known positions of the AES, it is obvious from Formulas (32.18) and (32.19) that the optimum figure is one in which the AES positions are observed on a trajectory passing through the zenith of the point, at the boundary of the zone of visibility. In this case, the length of the celestial chord is maximum, and its center is located at the zenith, i.e., at the minimum distance from the point. The difference in distances is then equal to zero. Thus, we may assume that the advance of this figure is essentially equal to zero. When the conditions deviate from optimum, the accuracy of calculating the position of the point to provide a substantial advance decreases sharply.

Based on the foregoing, the use of an elementary figure where the difference in distances is calculated to two known AES positions, and the direction is calculated to a third position [see Formula (32.18)], should be considered unfeasible.

\section{Calculation of the Position of the Point from the} Direction and Chord Length

We may conclude from analysis of the formula for estimating the accuracy of the elementary figure for calculating the position of the point - from the direction of the chord and its length, obtained from the measured distance from the point to the AES (32.13) - that the error in the position of the point is minimal at a minimum value of the ratio between the unmeasured distances to the AES and the measured distances. 
The optimum observation conditions may be achieved if the AES position, to which the distance is measured, is located in a plane passing through the chord and the center of the Earth, and the measured distance is maximum for a given AES altitude at a given minimum angle of AES elevation above the level of the observation point. Under these conditions, the error in the chord length, which determines the accuracy of the position of the point, will be

$$
\frac{m_{D}^{2}}{D^{2}}=\frac{D^{2}+\rho_{i h_{\max }^{2}}+\rho_{j k}^{2}}{2 \rho_{i h_{\max }}^{2} \sin ^{2} \varphi k} m_{\rho}^{2}
$$

By varying the values of $m_{D} / D$ by this formula, we can obtain the optimum chord length $D_{\text {opt }}$ and the corresponding values of $\rho_{j k}$ and $\varphi_{k}$, which are its functions, for each combination of given $\mathrm{H}$ and $\alpha$.

Thus, for $\alpha=20^{\circ}$, a graph of the dependence of $\mathrm{D}_{\text {opt }}$ on $\mathrm{H}$, i.e., a graph of the optimum values of the ratio of chord length to AES altitude, is presented in Figure 63. Incidentally, it is obvious from consideration of the graph that the optimum chord length in the given figure is close to the optimum chord length in the figure for calculation of chord direction.

In the case when chord length is less than optimum for a given AES altitude, the suitable shape of the figure may be characterized by the optimum value of the angle of elevation of the AES, to which the distance of the observation point above the horizon is measured. 


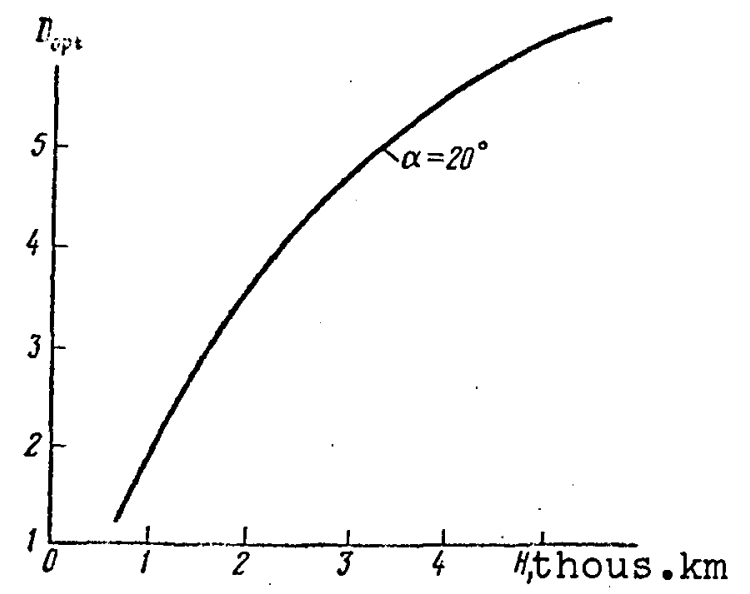

Figure 63.

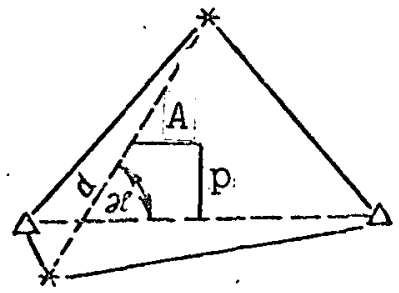

Figure 64.

The graph of $\alpha_{\text {opt }}$ for the

calculated range of chord length values (D) at a given AES altitude may be constructed similarly to the graph presented in Figure 56 and used for projection of the observations.

The figure for calculating the position of the point from the direction and length of the chord, when combining synchronous photographic observations and measurements of the difference in distances to the AES positions, is another matter.

The shape of the figure may be characterized by several parameters of the schematic projection of the figure on the Earth's surface (Figure 64):

- by the angle between the chord directions and the AES path $(\kappa)$;

- by the length of the celestial chord (d);

- by separation of the projection of the celestial chord center from the center of the ground chord along (A) and perpendicular to $(p)$ its direction. The best method for calculating the optimum parameters of the elementary figure to provide the condition $m_{D} / D=m i n$ would be, for example, at a given AES altitude, solution of a system of equations 


$$
\begin{gathered}
\frac{\partial\left(\frac{m_{D}}{D}\right)}{\partial D}=0 ; \quad \frac{\partial\left(\frac{m_{D}}{D}\right)}{\partial x}=0 ; \quad \frac{\partial\left(\frac{m_{D}}{D}\right)}{\partial d}=0 ; \\
\frac{\partial\left(\frac{n_{D}}{D}\right)}{\partial \mathrm{A}}=0 ; \quad \frac{\partial\left(\frac{m_{D}}{D}\right)}{\partial \cdot \mathrm{A}}=0 .
\end{gathered}
$$

However, obtaining a system of such equations and solutions of it are very complicated.

It follows from an analysis of the formula for the error in chord length (32.15) that one of the requirements for the optimum shape of the figure is to provide a maximum value of the difference in distances to the AES. When the distances to the AES are equal, the error in the chord length is equal to infinity, 1.e., the chord length, and consequently, the position of the point as well are not calculated. Combination of this requirement with that for maximum advance of the figure permits us to assume that the shape of the figure will be optimum if the condition is fulfilled that the distance from each of the points to at least one AES position is maximum (37.1).

Let us consider such a figure with different values of the angle $\kappa$ between the chord direction and the AES path.

Diagrams of the figures considered, as well as their comparative data for $H=0.2 R$ and $\alpha_{\min }=20^{\circ}$ are presented in Table 2 .

Analysis of the figures presented in Table 2 leads to the following conclusions.

The first figure, which is optimum for calculating the chord direction, makes it impossible to calculate the position of the point, because $\Delta \rho=0$ and the chord length may not be obtained. 
TABLE 2

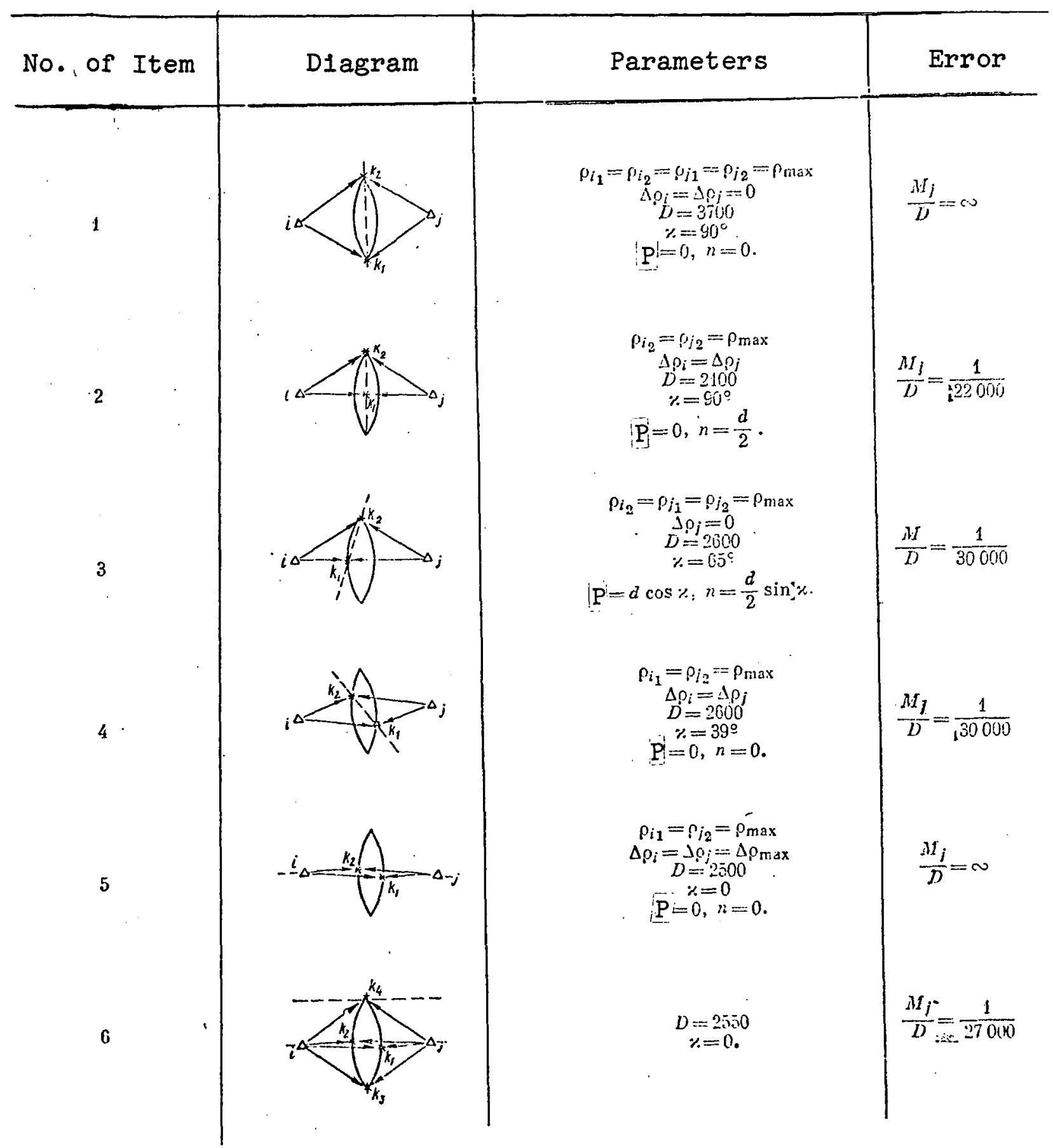


The most suitable value and shape of the second figure are calculated by the optimum chord length, because the length of the celestial chord $d=f(D)$.

The third figure is less suitable than the second, because the difference in distances in this figure to the AES positions is equal to zero and, consequently, the differences in distances in this figure may be measured only from another point.

The most suitable dimensions and shape of this figure are also calculated by the optimum chord length, because $d=f_{1}(D)$ and $k=f_{2}(D)$.

The most suitable value and shape of the fourth figure are calculated by the optimum chord length and the value of angle $k$, because $D=f(K)$.

The fifth figure, where the positions of the AES and of the 1183 points are located in a single plane, passing through the center of the Earth, makes it impossible to calculate the chord direction, and consequently, the position of the point. However, if the chord direction has. been calculated previously (only from simultaneous photographic observations of other AES positions), this type of figure is optimum in terms of the accuracy of calculating the chord length.

In conclusion, a construction is presented which is a combination of the first and fifth figures, in which four rather than two AES positions are observed (the sixth figure).

A comparison of the advantages of these figures may be made on the basis of the graphs (Figure 65), constructed for $\alpha=20^{\circ}$, $m_{B}=2^{\prime \prime}$, and $m_{\Delta \rho} / H=1: 100,000$. 


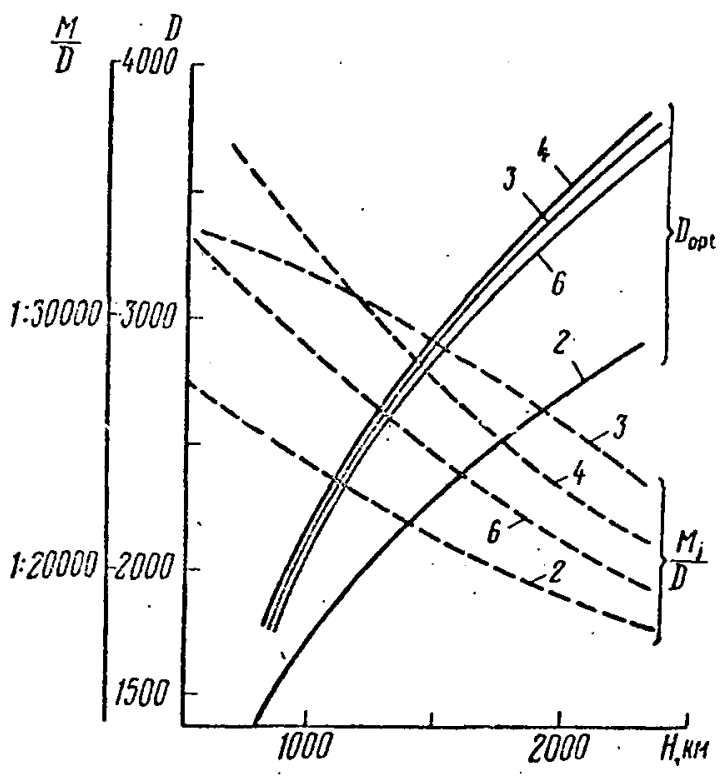

Figure 65.

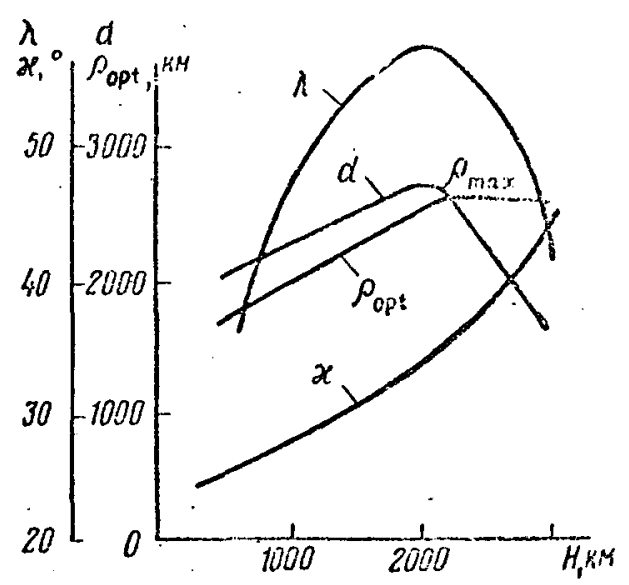

Figure 66 .

It is obvious from the graphs and Table 2 that the second figure is less suitable (it has a

less optimum chord length and a greater relative error in the point) than the remaining figures, which essentially provide identical results.

Upon selection of the optimum type of figure for a specific chord, we are forced to consider the real value of angle $k$ between the chord direction and the direction of the AES path, calculated by the angle of orbital inclination of the AES to the equator and by the mean latitude of the chord. Therefore, the range of application of the elementary figures of the optimum shape is IImited.

With given orbital parameters of the AES, we can recommend the following as optimum:

- the sixth figure at $0<k<15^{\circ}$,

- the fourth figure at $15^{\circ}<\kappa<45^{\circ}$, 
- the third figure at $45^{\circ}<\kappa<75^{\circ}$, and

- the second figure at $75^{\circ}<\kappa<90^{\circ}$.

When the given chord length is less than optimum, the most suitable figure is calculated by the optimum distance from the point to the AES position ( $\rho_{\text {opt }}$ ).

The nature of the variation of the value $\rho_{\text {opt }}$, as well as 1184 of other parameters, characterizing the shape of the fourth figure $d, k$ and $\lambda$, with variation of the value $D / H$, is shown in the graphs (F1gure 66), and the variation of the absolute $\left(M_{j}\right)$ and relative $\left(M_{j} / D\right)$ errors in the position of the calculated point as $D$ increases at $H=0.2 R$ is shown in Figure 67 .

The graphs indicate that where $D<D_{\text {opt }}$, the value of all parameters increases. It continues at $D \geq D_{o p t}{ }^{k}$, and $d$ and $\lambda$ decrease, while $\rho_{\text {opt }}$ remains equal to $\rho_{\max }$.

The construction of such graphs for the proposed chord lengths of the projected triangulation may be used as the basis for selecting the optimum figures.

Figures with an Excess Number of Measurements

When deriving formulas for estimating the accuracy as a function of the number of excess measurements, we considered the increase in the number of measurements in the figure, which alters its geometric shape, as the most general version. 


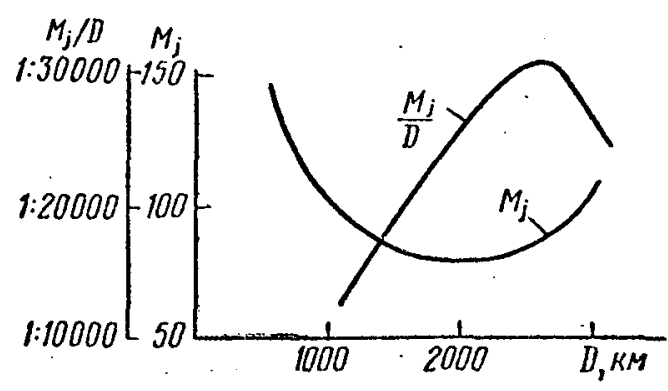

Figure 67.

We considered the optimum shapes and dimensions for the elementary figures. Moreover, it was established that the figures which differ slightly from the elementary figures by the number of measurements, are formed with observations of individual AES positions, whereas during prolonged observation sessions, the figures from which the positions of the points are calculated may contain a considerable number of excess measurements.

Since the sighting target in space triangulation is the instantaneous position of a satellite, moving according to a specific law, the frequency of the AES passing through the optimum locations for its observation, which we have selected for the elementary figures, will in most cases be considerably less than the frequency of passing through the entire zone of the joint visibility of the AES from the calculated and starting points.

Consequently, in order to achieve a specific accuracy of the results of space triangulation within a limited observation time, the requirements on the optimum shapes of the figures may be contradictory to the requirement for the required number of measurements.

In order to establish the relationships between these requirements, let us consider the example of estimating the accuracy of the result of the simplest of the space triangulation figures - figures for calculating the chord direction upon observation of more than two AES positions from the ends of the chord. 


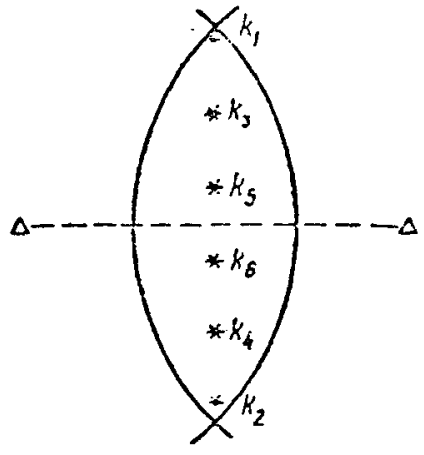

Figure 68.
Let six AES positions, equally distributed perpendicular to the chord direction (Figure 68), be observed in the zone of joint visibility from the points. Moreover, two AES positions ( $k_{1}$ and $k_{2}$ ) are located at optimum positions for the elementary figure. We may assume that an interval equal to one sixth of the zone of visibility length corresponds to each of the six AES positions.

Since the frequency of AES passages through each interval should be assumed to be identical, as much time is required for an equal number of observations of all six AES positions as is necessary for the same number of AES observations at two optimum positions.

Using Formula (33.8), we may calculate the error in the chord direction during observation of all AES positions $\left(m_{6}\right)$ and of two optimum positions $\left(\mathrm{m}_{2}\right)$. The ratio of the values of the errors is equal to: $m_{6}: m_{2}=1: 1.44$.

If it were necessary to carry out the same-number of observations to calculate the chord direction in the given case, the AES positions $k_{1}$ and $k_{2}$ would be observed three times, and the ratio of the errors would be equal to $\mathrm{m}_{6}: \mathrm{m}_{2} / \sqrt{3}=1: 0.83$. It is obvious that in the given case the error, obtained as a result of AES observations at optimum positions, is less. However, three times as much time is required to achieve it, because the frequency of the AES passing through the intervals, corresponding to positions $k_{1}$ and $k_{2}$ and comprising one third of the total length of the zone of visibility, is three times less than the frequency of the AES passing through the entire zone of visibility. 
The result obtained indicates that the optimum condition to achieve the required accuracy over a limited observation time may be observation of AES positions, uniformly distributed in the zone of mutual visibility of the points.

The area of the mutual visibility zone may be approximately represented by the expression

$$
\begin{aligned}
S=0,53\left(\sqrt{\rho_{\operatorname{tax}}^{2}-H^{2}}-\frac{D}{2}\right)^{3 / 2}\left[3 \sqrt{\sqrt{\operatorname{lin}_{2}^{2}-H^{2}}+\frac{D}{2}}+\right. \\
\left.+2 \sqrt{2 \sqrt{\rho_{\max }^{2}-H^{2}}}\right] .
\end{aligned}
$$

The areas of the mutual visibility zones at different AES altitudes may be obtained in order to establish the optimum parameters of the figures, considered in the example, for calculating the chord direction at a given chord length and a known minimum value of AES elevation above the level of the observation point.

Since the element of the area, corresponding to observation of a single AES position, is given, we may obtain the number of AES positions, uniformly distributed throughout the visibility zone, for the different AES altitude values.

We may now look for the minimum value of the total error in the chord direction, obtained as a result of observing ali $n$ AES positions in the zone of visibility, as a function of AES altitude for each chord.

For these purposes, Expression (33.7) may be simplified

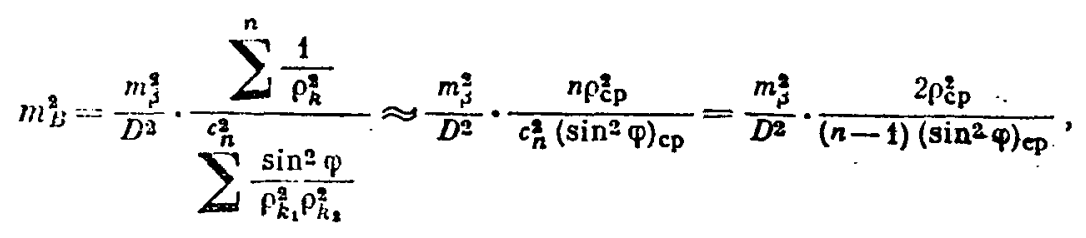


where the average values of $\rho_{c p}^{2}$ and $\left(\sin ^{2} \varphi\right)_{c p}$ may be obtained each time for the entire zone.

Analysis of Expression (37.11) indicates that the ratio $\rho_{c p}^{2} /(n-1)$, 1.e., the ratio of the increase in the square of the distance to the AES to the zone of mutual visibility, basically changes as AES altitude varies. On the basis of the variation

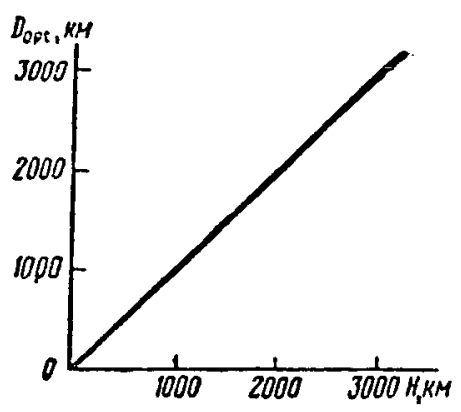

Figure 69. of this ratio, we may establish the optimum AES altitude for each chord length, providing a minimum direction error when observing AES positions, uniformly distributed within the zone of visibility. The graph of this dependence for $\alpha_{\mathrm{min}}=$ $20^{\circ}$ is presented in Figure 69. It is obvious that the optimum ratio of chord length $D$ and AES altitude $H$ differs from that required for the elementary figure (see Figure 55).

Thus, consideration of the variations in the requirements for the optimum parameters of the figure for calculating the chord direction with excess measurements leads to a number of important conclusions.

With a given number of observations of AES positions, which are required to obtain the result (which may be, for example, with limited operation of the observation equipment), observation of AES, located at positions corresponding to the optimum shapes of an elementary figure, will be preferable. 
Observations of the excess AES positions at locations unsuitable for obtaining the results from an elementary figure, in combination with AES observations at optimum positions for the elementary figure, have a negligible effect on the decrease in accuracy.

The optimum condition, for a given time for the observation session when the accuracy of one observation is low and a large number of observations is necessary to obtain the required accuracy, will be observation of all AES positions, uniformly distributed in the zone of mutual visibility from the points.

In some cases, the AES observation zones must be decreased. This may be done optimally when the condition of approximating the observed AES positions to the most suitable ones for the elementary figure is followed. The dimensions of the zone of mutual visibility, in which the positions of the observed AES positions are uniformly distributed, may be decreased without a loss of accuracy by increasing the elevation of the observed AES above the horizon. Consequently, the visibility zone for such cases is established on the basis of the value $\rho_{\text {opt }}$, i.e., the optimum distance from the point to the AES, rather than on the basis of the value of $\rho_{\max }$. The dependence of the variation of $\rho_{\text {opt }}$ as the ratio $\mathrm{H} / \mathrm{S}$ increases may also be represented graphically. Moreover, by increasing the elevation, we weaken the effect of refraction on the accuracy of measuring the directions from the point to the AES positions.

The conclusions obtained, as well the proposals for establishing the optimum conditions to obtain chord direction during the observation of an excess number of AES positions, may also be extended to other figures, for example, different types of intersection. 
For figures whose excess measurements are unrelated and whose error is calculated, for example, by Expression (32.15), the requirement of obtaining the necessary accuracy within a given time also entails observations of AES positions, uniformly distributed in the zone of mutual visibility from the observation points.

In order to calculate the optimum dimensions of the zone of mutual visibility in the given case, it is expedient to use the criterion of the minimum ratio between the mean error for the zone and the area of this zone, proposed by Lambeck for calculation of chord length by laser measurements of the distances to the AES [58].

\section{Optimum Conditions for the Transfer of Coordinates} in a Space Triangulation Series

The criterion of the optimum conditions for continuation of a space triangulation series may be the minimum relative error in the position of the end of the series, i.e., the ratio of the error in the position of the last point of the series $\left(M_{n}\right)$ to its length (L)

$$
\frac{M_{n}}{L}=\min
$$

provided that the number of points of the series is minimum.

The optimum shape for the elementary figure of a space triangulation serles, besides the conditions: $\mathbb{M}_{j} / A=\min$ and $A / b=\max$, should satisfy the requirement of constant advance. This requirement may be satisfied by several diagrams for a series, shown in Figures $70-75$. 

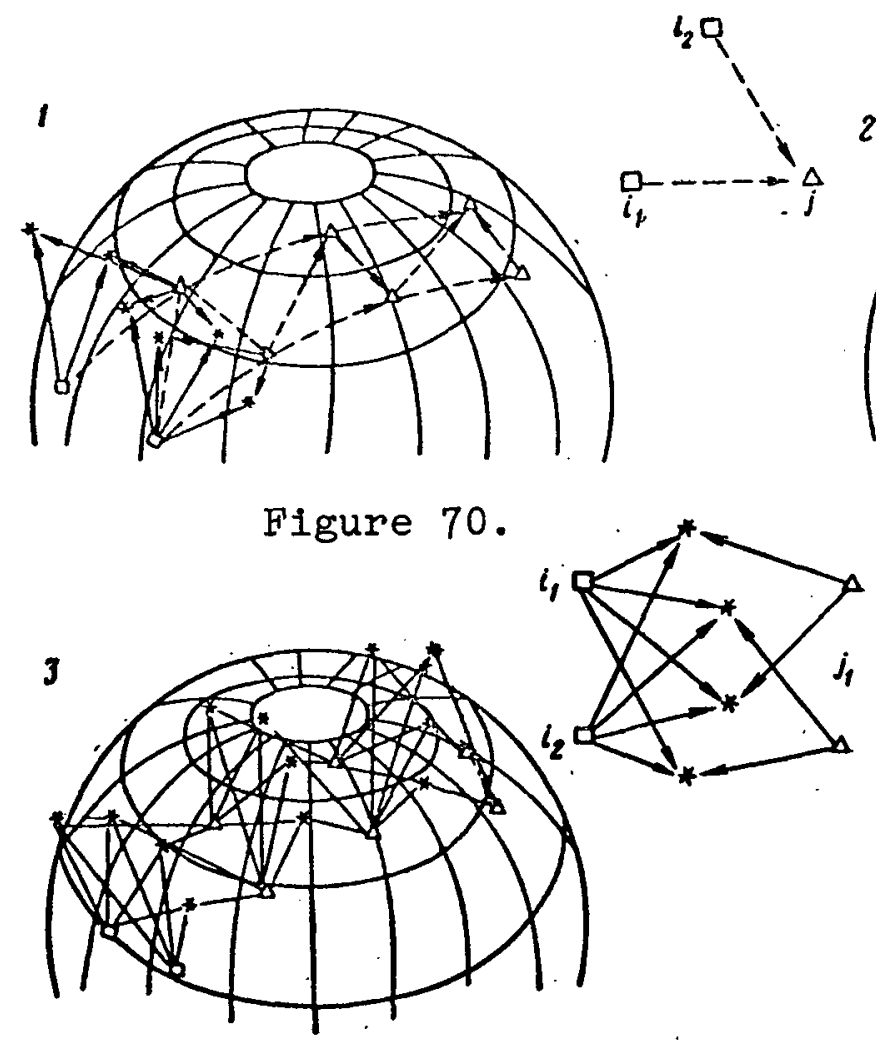

Figure 72 .

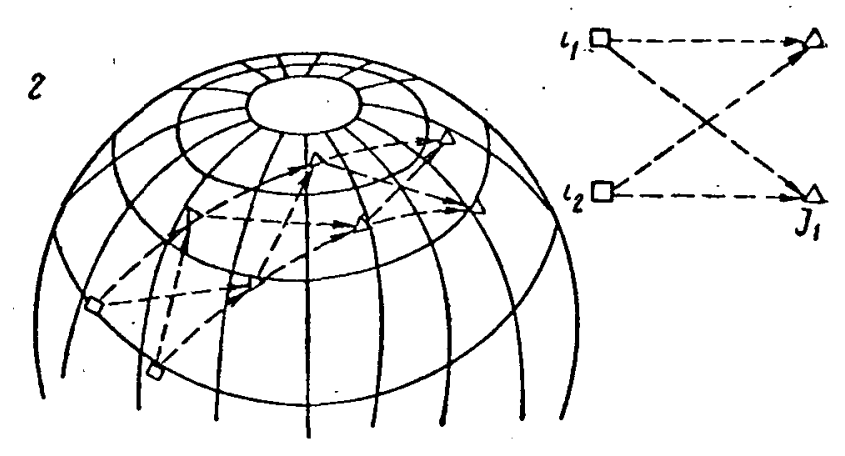

Figure 71

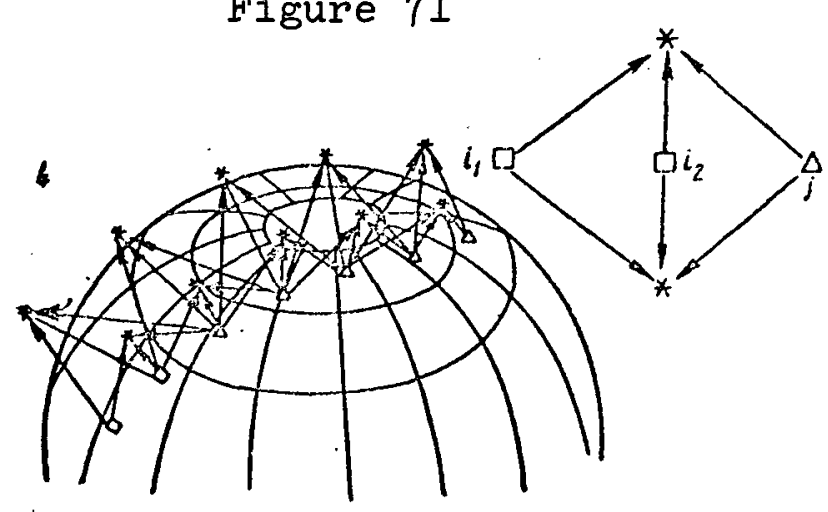

Figure 73.

The optimum shape of the figure in the first diagram is intersection of the chord directions, whlch cross at an angle of $60^{\circ}$. Its dimensions are calculated by the optimum chord length for a given AES altitude (Figure 70 ).

An elementary figure with a relative advance equal to $A / b \approx$ 2.2, 1s optimum for the second and third diagrams for the series (Figures 71 and 72). In this case the errors of both figures are similar and do not exceed the doubled error of the first figure, and the absolute advance differs only slightly from the maximum advance.

The optimum shape and dimensions of the figure for the fourth diagram of the series (F1gure 73) are calculated by the optimum distance between the points, which may be found from a solution of the equation $\mathrm{dM}_{j} / \mathrm{dD}=0$ for a given value of AES 


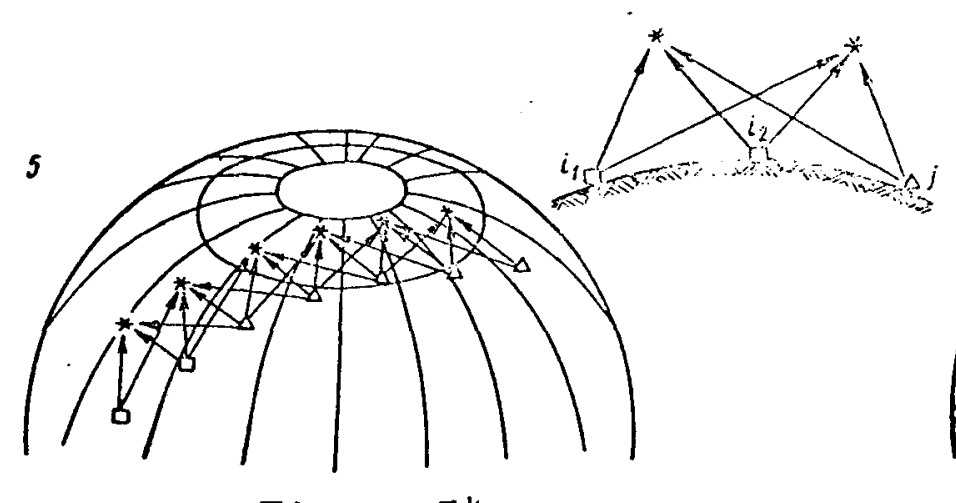

Figure 74 .

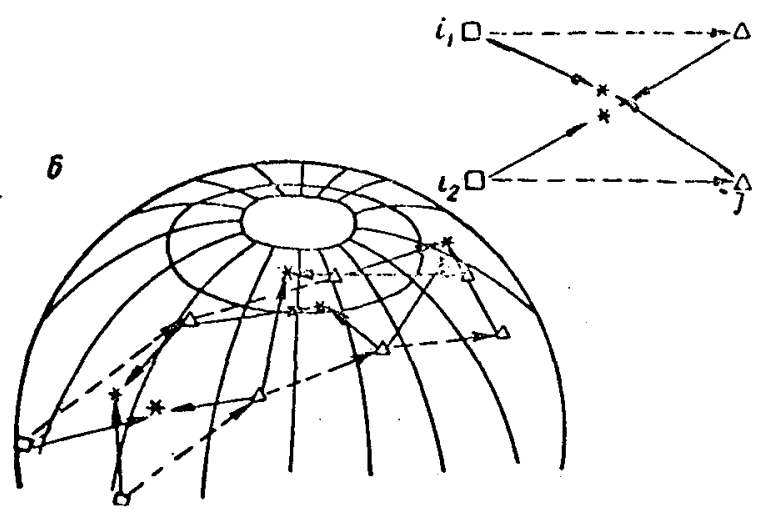

Figure 75 .

altitude. In this case, the error in the position of the point is calculated by the formula

$$
\begin{aligned}
M_{j}^{2}=: & \left\{\frac{A-B+\rho_{\ln }^{2}}{3\left[1-\frac{(A-B)}{\left.4(A-B) \rho_{\max }^{2}-D^{2}\right)^{2}}\right]}+\rho_{\max }^{2}\right\} \times \\
& \times \frac{m_{j}^{2}}{1-\left[1-\frac{2 \cdot R+H)^{2}}{\rho_{\max }^{2}}+\frac{B^{2}}{2 R^{2} \rho_{\max }^{2}}\right]^{2}},
\end{aligned}
$$

where

$$
\begin{aligned}
& A=(R+H)^{2}+R^{2}, \\
& B=\frac{2 R^{2}\left(A-\rho_{\max }^{2}\right)}{2 R^{2}-D^{2}} .
\end{aligned}
$$

The shape and dimensions of the standard elementary figure of the fifth diagram, shown in Figure 74 in the projection onto the plane passing through the points, the AES positions and the center of the Earth (which also contains the characteristics of the given figure), are clearly calculated by the distance between the points, dependent on AES altitude, as follows from the expression

$$
\begin{gathered}
D^{2}=2 R^{2}\left[1-\cos \frac{2}{3} \times\right. \\
\left.\times\left(\arccos \frac{(R+H)^{2}+R^{2}-\rho_{\max }^{2}}{2(R+H) R}\right)\right] .
\end{gathered}
$$




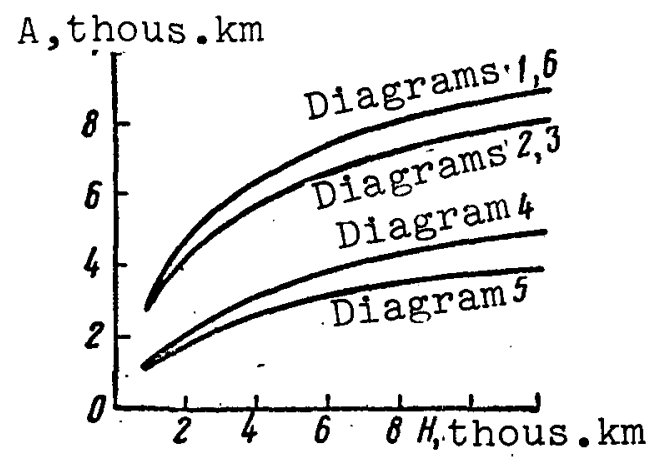

Figure 76.
Figures, formed by intersection of the chord direction with the synchronization plane (Figure 75), are used in the last and sixth diagram for construction of the series. The use of such figures may be required to achieve an advance equal to or greater than the optimum chord length.

In this case, the maximum distance between the points through which the synchronization plane passes is calculated by Expression $(37.7)$.

The dependence of the advance on AES altitude for these figures is shown in Figure 76 .

On the basis of (38.1), Expression (34.1) for the error in the position of the $n \frac{t h}{n}$ point of the series indicates a preference for a series from those figures which provides a minimum coefficient of the increase in error $K$.

When creating a space triangulation series, the figures will not be essentially elementary, but will contain a considerable number of measurements. However, the optimum dimensions of the elementary figures may be used for the comparative characteristics of series constructed from these figures. This problem is especially timely for space triangulation series, constructed from photographic observations, since the range of figures in such a triangulation is large, and the results depend mainly on the shape of the figures. 


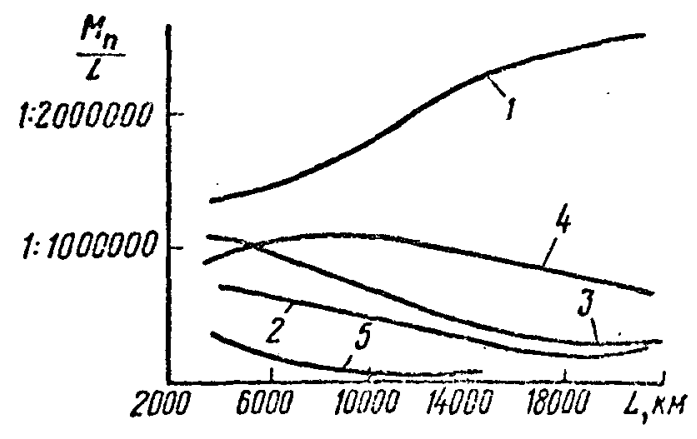

Figure 77 .

Establishing which diagrams are more suitable for a space triangulation series may be done by considering graphs which indicate the variation of relative error in calculating the position of the point of the series as a function of the length of the series $\left(M_{n} / L\right)$.

Such graphs are presented in Figure 77 for series of figures of optimum shape and value (at $\alpha=20^{\circ}$, $H=0.2 R$, and $m_{B}=I^{\prime \prime}$ ) for the five diagrams shown in Figures $70-74$.

Space triangulation series based on the first and fourth construction diagrams are recommended for more economical transfer of coordinates. The fifth diagram may be used in rare cases, dictated by physical and geographical conditions.

When constructing space triangulation series from combining observations, sequential use of the figures for calculating the point from the direction and length of the chord, when combined photographic observations both with measurement of the distances and with measurement of the differences in distances to the AES positions, has a number of considerable advantages compared to sequential use of figures, which calculate the AES positions, and then - the position of the point. The primary advantage is the fact that such figures require only a single starting point, which makes it possible to form networks from chords and chords similar to polygonometric ones. The secondary advantage is the possibiltty of calculating the position of the point from radio engineering observations at a single point - at any end of the chord. 


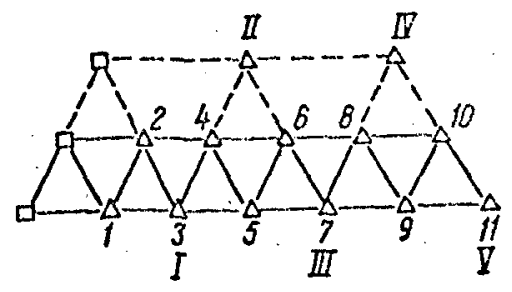

Figure 78 .

In the extension of a coordinate system, space triangulation series, compiled from figures of low value, with an optimum ratio of distances between the points and AES altitude, are preferable. This concept has already been expressed by Batrakov [3].

Thus, for example, when the errors in the common points of two series, constructed by the first diagram with an optimum ratio of AES altitude and advance, equal to 3,000 and $6,000 \mathrm{~km}$, respectively (Figure 78), and presented in Figure 79, are compared, it

is obvious that the relative error

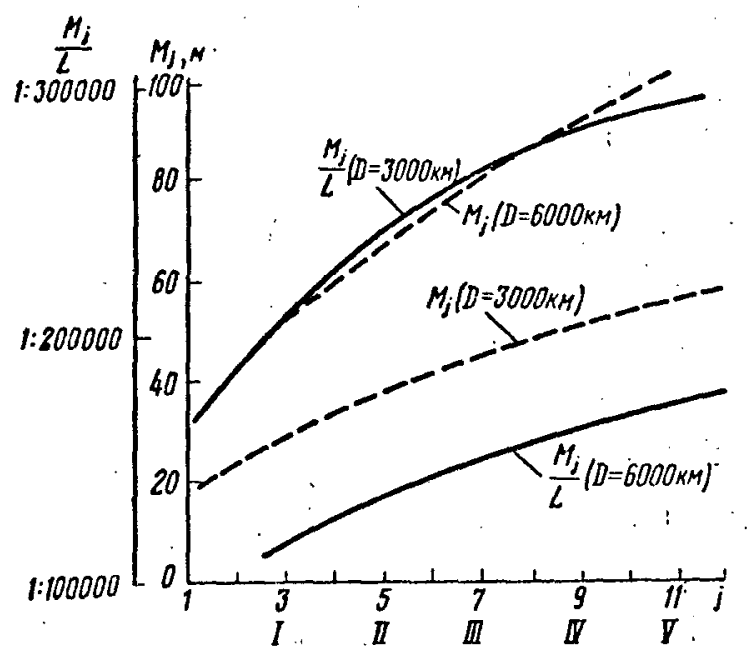

Figure 79. in the points of the first series is two times less. It is obvious that this same concept is also valid for extension of a coordinate system to a specific area by constructing a continuous space triangulation network.

It should be said in conclusion that figures of the first diagram (see Figure 70) - i.e., equilateral triangles, constructed from the chords of a length which

is optimum for the given AES altitude - may be used to construct a continuous space triangulation network, which requires retaining the value of the elementary figures in any direction. 


\section{Optimum Combinations of Measurements in Continuous Space Triangulation Networks}

The problems of the effect of the geometric shape of space triangulation figures are more timely for networks in which only the angular or only the linear values were measured.

Let us evaluate the effect of the geometric shape of the network in terms of the error in the position of the $j \frac{\text { th }}{\text { point }}$

$$
M_{I}=\mu \sqrt{q_{x_{l}}+q_{y_{l}}+q_{z_{l}}}
$$

where $q_{x_{j}}, q_{y_{j}}$, and $q_{z_{j}}$ are calculated by Formulas (35.31) $(35.34)$.

Certain assumptions with respect to the shape of the network $/ 193$ must be introduced for further analysis. We first assume that all directions, measured from a single point, are uniformly distributed with respect to the coordinate axes. In this case $\sum l^{2}=\sum m^{2}=\sum n^{2}=\sum q^{2}$ and, consequently, taking the fact into account that $\sum l^{2}+\sum m^{2}+\sum n^{2}=N$, we obtain $\Sigma q^{2}=N / 3$. Formulas (35.31) will then assume the form

$$
q_{x_{j}}=q_{v_{j}}=q_{z_{i}}=\frac{3}{2} \cdot \frac{p^{2}}{N}\left(1+\frac{2}{N}\right) .
$$

By comparing Expressions (39.2) and (35.31), we may conclude that, if the directions to the AES in the network are distributed uniformly with respect to the coordinate axes, the accuracy of calculating the points in this network is reduced by approximately $\sqrt{3 / 2}$ compared to a network in which three elements (1.e., $\gamma, \delta$, and $\rho)$ are measured. 


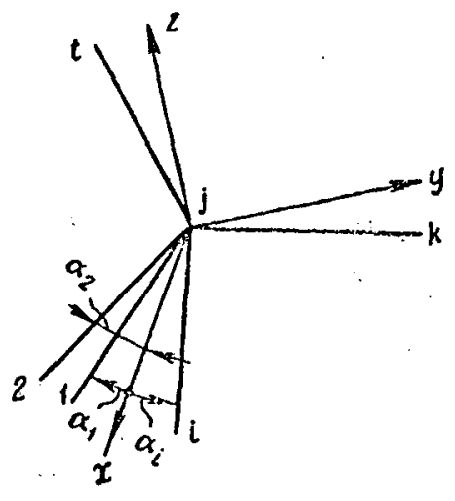

Figure 80 .

Now let some point in the network be calculated such that all directions, measured from a given point, are similar to each other. Let us introduce a coordinate system such that the directions are located close to the $\mathrm{X}$-axis, and the origin of the coordinates colncldes with the calculated point $\mathrm{f}$ (Figure 80 ). Let us denote the angles, composed of directions 1, 2 , $\ldots, 1$ with the $X$-axis, by $\alpha_{1}, \alpha_{2}, \ldots, \alpha_{f}$. We will then have

$$
\begin{gathered}
1-l_{1}^{2}=\sin ^{2} \alpha_{1}, \\
1-l_{2}^{2}=\sin ^{2} \alpha_{2}, \\
\cdot \cdot \cdot \cdot \cdot \\
1-l_{l}^{2}=\sin ^{2} \alpha_{l},
\end{gathered}
$$

$$
N_{t}-\sum l_{i}^{2}=\sum \sin ^{3} \alpha_{l}
$$

$$
\text { Assuming that } \begin{aligned}
\alpha_{1}= & \alpha_{2}=\ldots=\alpha_{1}, \text { we obtain } \\
& N_{l}-\sum l_{i}^{2}=N_{i} \sin ^{2} \alpha .
\end{aligned}
$$

For angles formed by these same directions $1,2, \ldots, 1$, with the $Y$ - and $Z$ - axes, due to the smallness of angles $\alpha_{1}$, we may assume that $m_{1} \cong n_{1} \cong 0$, and, consequently

$$
N_{l}-\sum m_{i} \approx N_{i}-\sum n_{i}^{2}=N_{i} .
$$


Taking these assumptions into account, Formulas (35.1) may be written in the form

$$
q_{x_{j}}=\frac{\rho^{2}}{N \sin ^{2} \alpha}\left\{1+\frac{3 N(1-l)}{2 N^{2} \sin ^{2} \alpha}\right\}
$$

But

$$
l^{4}=\left(1-\sin ^{2} \alpha\right)^{2}=1-2 \sin ^{2} \alpha+\sin ^{4} \alpha,
$$

and therefore,

$$
\begin{gathered}
q_{x_{i}}=\frac{\rho^{2}}{N \sin ^{2} \alpha}\left(1+\frac{3}{N}-\frac{3}{2 N} \sin ^{2} \alpha\right), \\
q_{y_{j}}=q_{z_{j}}=\frac{\rho^{2}}{N}\left(1+\frac{3}{2 N}\right) .
\end{gathered}
$$

The formula for the error in position $\mathrm{j}$, obtained by the weight coefficients (39.7) and (39.8), will have the form

$$
M_{j}^{2}=\mu^{2} \frac{\rho^{2}}{N \sin ^{2} \alpha}\left(1+\frac{3}{N}+\frac{4 N+3}{2 N} \sin ^{2} \alpha\right)
$$

Let us now consider a case when at least one direction, (for example, $j k$ in Figure 80) comprises a "good" angle with each of the remaining directions (i.e., close to $90^{\circ}$ ). Assuming that $\alpha_{j k}=90^{\circ}$, and $\sin \alpha_{j k}=1$, we obtain the following expressions for the weight coefficients:

$$
\begin{gathered}
q_{x_{j}}=\frac{\rho^{2}}{1+(N-1) \sin ^{2} \alpha}\left\{1+\cdot \frac{3}{2} \cdot \frac{1}{\left[1+(N-1) \sin ^{2} \alpha\right] N}+\right. \\
\left.+\frac{3(N-1) \sin ^{2} \alpha}{\left[1+(N-1) \sin ^{2} \alpha\right] N}\right\},
\end{gathered}
$$




$$
\begin{gathered}
q_{y_{j}}=\frac{\rho^{2}}{(N-1)+\sin ^{2} \alpha}\left\{1+\frac{3}{2} \cdot \frac{N-1}{\left[(N-1)+\sin ^{2} \alpha\right] N}+\right. \\
\left.+\frac{3 \sin ^{2} \alpha}{\left[(N-1)+\sin ^{2} \alpha\right] N}\right\} \\
q_{z_{j}}=\frac{\rho^{2}}{N}\left(1+\frac{3}{2 N}\right) .
\end{gathered}
$$

We note than in Formulas $(39.7)$ - (39.11) the terms are discarded which contain a sine of a small angle in the fourth power in the numerator.

Let us further assume that at least two directions (directions $j k$ and $j t$ in Figure 80 ) comprise "good" angles with the remaining directions. This will mean that $\alpha_{j k}=\alpha_{j t} \approx 90^{\circ}, \sin \alpha_{j k} \approx \sin \alpha_{j t}$ $\approx 1$, and then - if terms containing $\sin ^{4} \alpha$ are not retained the formulas for the welght coefficlents will assume the form

$$
\begin{gathered}
q_{x_{l}}=\frac{\rho^{2}}{2+(N-2) \sin ^{2} \alpha}\left\{1+\frac{3}{\left[2+(N-2) \sin ^{2} \alpha\right] N}+\right. \\
\left.\quad+\frac{3(N-2) \sin ^{2} \alpha}{\left[2+(N-2) \sin ^{2} \alpha\right] N}\right\} \\
q_{y_{j}}=q_{z_{l}}=\frac{\rho^{2}}{(N-1)+\sin ^{2} \alpha}\left\{1+\frac{3}{2} \cdot \frac{N-1}{\left[(N-1)+\sin ^{2} \alpha\right] N}+\right. \\
\left.+\frac{\because 3 \sin ^{2} \alpha}{\left[(N-1)+\sin ^{2} \alpha\right] N}\right\} .
\end{gathered}
$$

The values of $\sqrt{1 / p_{j}}$, where the value of $1 / p_{j}$ is calculated from the expression: $1 / p_{j}=q_{x_{j}}+q_{y_{j}}+q_{z_{j}}$, are presented in Table 3. The weight coefficients of $q_{x_{j}}, q_{y_{j}}$, and $q_{z_{j}}$ are 
TABLE 3

\begin{tabular}{c|c|c|c|c}
\hline \multirow{2}{*}{$\alpha$} & \multicolumn{4}{|c}{$\begin{array}{c}\text { Directions are } \\
\text { distributed } \\
\text { uniformly }\end{array}$} \\
\cline { 2 - 5 } & $\mathrm{N}=6$ & $\mathrm{~N}-1=5$ & $\mathrm{~N}-2=4$ & \\
\hline 1 & 2 & 3 & 4 & 5 \\
\hline $15^{\circ}$ & 2.04 & 1.24 & 1.08 & 1.00 \\
$10^{\circ}$ & 2.90 & 1.26 & 1.10 & 1.00
\end{tabular}

calculated as a function of the assumed geometric conditions from Formulas (39.7), (39.8) (the third graph), (39.10)-(39.12) (the third graph), (39.13)-(39.14) (the fourth graph) and (39.2) (the fifth graph).

The data of Table 3 indicate that, if even two directions form "good" angles with the remaining directions, the loss of accuracy is essentlally negligible. In continuous networks, there are no cases when all angles, made by the directions measured from a given point, will be small, but this case (the second graph of Table 3) leads to a great decrease in accuracy. Therefore, if part of the directions, belonging to a given point, form small angles among themselves, the accuracy of calculating the point is not reduced.

Similar calculations may be carried out for networks in which only the distances to the AES are measured. The values of $\sqrt{1 / p}$ for this case are presented in Table 4.

It 1 s obvious from Table 4 that the effect of the shape of the network is more strongly manifested for networks, constructed only by measured distances to the AES. In this case two sides, intersecting at "good" angles, are required as a minimum. 
TABLE 4

\begin{tabular}{|c|c|c|c|c|}
\hline \multirow[t]{2}{*}{$\alpha$} & \multicolumn{3}{|c|}{$\begin{array}{c}\text { Number of directions, forming small } \\
\text { angles at a given point }\end{array}$} & \multirow[t]{2}{*}{$\begin{array}{l}\text { Directions are } \\
\text { distributed } \\
\text { unfformly }\end{array}$} \\
\hline & $N=6$ & $N-1=5$ & $N-2=4$ & \\
\hline $15^{\circ}$ & 3.12 & 2.53 & 1.80 & 1.65 \\
\hline $10^{\circ}$ & 4.70 & 3.60 & 1.95 & 1.65 \\
\hline
\end{tabular}

To increase accuracy, preference should be given to networks in which all three elements are measured. However, this will hardly be the best engineering solution of the problem. Let us illustrate this position by an example. Let $N$ directions be measured at some point, and let the distances also be measured by $t$ directions. Then $(35.30)$ assumes the form

$$
\begin{aligned}
& N_{i}=\mu \rho \sqrt{\frac{1}{p_{J}}}=\mu \rho \sqrt{1+\frac{t+\left[(N-t)-\sum q^{4}\right]}{t+\left[(N-t)-\sum q^{2}\right]} \times} \\
& \times \sqrt{\frac{1}{t+\left[(N-t)-\sum l^{2}\right]}+\frac{1}{t+\left[(N-t)-\sum m^{2}\right]}+\frac{1}{t+\left[(N-t)-\sum n^{2}\right]}} .
\end{aligned}
$$

or with uniform distribution of the lines with respect to the coordinate axes

$$
M_{j}=\mu \rho \sqrt{\frac{3}{t+\frac{2}{3}(N-t)}\left\{1+\frac{\frac{8}{9} N+\frac{1}{9} t}{\left[t+\frac{2}{3}(N-t)^{3}\right]^{2}}\right\}}
$$

The reciprocal weights of the errors in the position, calculated by Formula (39.16) and for different values of $t$ (in this case $N$ is assumed equal to 6 ), are presented in Table 5 .

It is obvious from Table 5 that, if a single distance $(t=1)$ 1s measured from each ground point, the accuracy of calculating the points is increased by a total of $6 \%$, of two points - by $13 \%$, and finally, if the distances at the points are measured by all directions, an increase of accuracy by $24 \%$ may be expected. 


\section{TABLE 5}

\begin{tabular}{c|c|c|c|c}
\hline$t$ & $t=0$ & $t=1$ & $t=2$ & $t=N$ \\
\hline$\sqrt{1 / p_{j}}$ & 1.00 & 0.94 & 0.87 & 0.76
\end{tabular}

Thus, measurement of the differences by all lines of the space geodetic network, by considerably increasing the total number of operations, increases accuracy by only 1.2 times. Therefore, the main role of linear measurements should be not so much in increasing accuracy, as in representing and conserving the scale for the entire network. In connection with this, no attempt should be made to measure all lines, but only part of them, distributing them uniformly over all sections of the network.

The problem of the optimum number of measured sides and their distribution requires additional investigations. But since the errors in the measurements are localized in the section consisting of six to eight series of points, we may assume in the first approximation that the effect of linear measurements on determining the scale of a given section of the network will be approximately within these limits. Therefore, it is recommended that points for which distances are measured be arranged along six to eight sides of space triangulation.

At the same time, it is necessary to note that measurement of the differences is of great importance at those positions where, for some reason or other, the network has an unsatisfactory geometric shape. A radical increase in the accuracy of space triangulation in such sections may be achieved by an optimum combination of angular and linear measurements. 


\section{Calculation of the Optimum Characteristics of Space Triangulation Design}

Space triangulation design should contain a number of characteristics, necessary for its implementation, which may be divided into three groups.

The first group comprises the indicators of accuracy:

$I$ - measurement errors $\left(m_{\beta}, m_{\rho}, m_{\Delta \rho}\right)$,

2 - errors in calculating the triangulation elements $\left(m_{B}, m_{D}\right)$,

3 - errors in the position of triangulation points $\left(M_{f}\right)$ or relative errors in their common position $(v)$;

The second group will contain the geometric indicators:

4 - the distances between ground points (D),

5 - the orbital parameters of the AES ( $i, H)$,

6 - the distances to the AES $(\rho)$,

7 - the angles between the directions, planes, and chords $(\varphi, \lambda, \psi)$,

8 - the elevation of the AES above the level of the observation points $(\alpha)$;

The third group may include quantitative characteristics:

9 - the number of AES positions or directions from the observation point, or the number of synchronization planes required to obtain the result $(n)$, 
10 - the number of observed $\operatorname{AES}(s)$,

11 - the frequency of the AES passing through the zone of common visibility from the observation points ( $f$ ),

12 - the duration of total observation time (T).

Under different conditions for solution of the design problem, some characteristics will be given before, and others will be determined during, the design.

The most common case is a design based on the overall purpose for space triangulation, when only the average distance between the points and the accuracy of their calculation will be given. The design should then be determined from all remaining characteristics, up to selection of the observation equipment, providing the required accuracy of observations.

A design based on representation of the first, third, fourth, fifth, tenth, eleventh and twelfth characteristics will be the most practical. In this case, the agreement of the results of triangulation accuracy, provided by the design, and the time required for its creation should be checked.

The narrowest problem will be a priori calculation of the $\underline{198}$ accuracy of the position of the points in the network, when all characteristics, besides the third one, are given. The calculations here reduce to sequential use of the formulas for estimating the accuracy of the position of the points. Numerical inversion of the matrices of normal equations on an electronic computer may also be used for a more rigorous evaluation of the design.

To illustrate the latter problem, let us analyze one of the designs of a worldwide space triangulation network, proposed by the Soviet scientist, I. D. Zhongolovich [18]. 
The basic idea of this design consists in constructing a closed three-dimensional network of space triangulation triangles around the Earth, whose chords form a rectilinear polygon - an icosahedron. The design has the following technical characteristics: space triangulation is constructed by the method of chords, the number of calculated points is 12 , and the number of chords, convergent at each ground point, is identical and equal to 5, the chord lengths $D=6700 \mathrm{~km}$, the satellite altitude $\mathrm{H}=12,300 \mathrm{~km}$; the average distance between the ground points and the AES is $\rho=14,000 \mathrm{~km}$, which will correspond to the angle of intersection for a satellite of $\varphi=28^{\circ}$.

The author of the design assumes that, with a sufficient number of intersecting planes (synchronization planes), we can achieve an accuracy in calculating the spherical coordinates of the chords in the space network of about $1^{\prime \prime}, 1 . e ., m_{\varphi}^{\prime \prime}=m_{\Lambda} \cos \varphi=$ I". For these conditions, the error in the direction of the chord comprises $m_{B}^{\prime \prime}=1.4 "$.

Let us first calculate which number of synchronization planes should be obtained to achieve such accuracy. From Formula (37.11) we will have

$$
(n--1)=\frac{m_{\rho}^{2} 2 \rho_{\mathrm{c}}^{3}}{m_{B}^{2} D^{2}\left(\sin ^{2} \lambda_{\mathrm{cp}}\right.} .
$$

Assuming that $m_{B}=1 ", m_{B}=1.4 ",\left(\sin ^{2} \lambda_{c p}=0.3, D=6700, \rho=\right.$ $14,000)$ we obtain $n=16$.

Let us now estimate the errors of the points of the first series of the network shown in Figure 54 (points 2, 3, 4, 5, 6). The errors in the position of points in the networks with measured directions to the AES are calculated by Formula (35.31). 
The latter may be simplified, if we assume that the measured directions are uniformly distributed with respect to the coordinate axes. Then $\Sigma l^{2} \approx \Sigma m^{2}=\Sigma n^{2} \approx \Sigma q^{2}=N / 3$, and Formula (35.3I) assumes the form

$$
M_{i}=\mu \rho \sqrt{\frac{9}{2 N}\left(1+\frac{2}{N}\right)}
$$

When the chords are equated in the network, the formula for the error in the position is the following:

$$
M_{i}=m_{B} D \sqrt{\frac{9}{2 N}\left(1+\frac{2}{N}\right)} .
$$

The value of the error, calculated by Formula (40.3) will be identical for all points and will be

$$
M_{j}=\frac{1,4 \cdot 6700000}{206265} \cdot 1,13=52 x
$$

When deriving Formula (40.3), the errors in the coordinates are assumed equal to $\mathrm{M}_{\mathrm{x}_{j}}=\mathrm{M}_{\mathrm{y}_{j}}=\mathrm{M}_{\mathrm{z}_{\mathrm{j}}}=30 \mathrm{~m}$.

Professor I. D. Zhongolovich reduces the errors in the coordinates of the same points, obtained as a result of adjusting

\begin{tabular}{|c|c|c|c|c|}
\hline $\begin{array}{l}\text { No. of } \\
\text { point }\end{array}$ & ${ }^{M}{ }_{x_{j},},{ }^{\prime \prime}$ & ${ }^{M} y_{i},{ }^{\prime \prime}$ & $M_{z_{i}},{ }^{\prime \prime}$ & $M_{j}=\sqrt{M_{x_{j}}^{2}+M_{y_{j}}^{2}+M_{z_{i}}^{2}}, x_{0}$ \\
\hline $\begin{array}{l}2 \\
3 \\
4 \\
5 \\
6\end{array}$ & $\begin{array}{l}30 \\
24 \\
44 \\
44 \\
24\end{array}$ & $\begin{array}{l}25 \\
42 \\
34 \\
34 \\
42\end{array}$ & $\begin{array}{l}26 \\
30 \\
32 \\
32 \\
30\end{array}$ & $\begin{array}{l}47 \\
57 \\
64 \\
64 \\
67\end{array}$ \\
\hline
\end{tabular}
the network model (Table 6), which corresponds to its design.

TABLE 6 
The data given in Table 6 indicate that the assumption about the uniform distribution of chords with respect to the coordinate axes, on the basis of which Formula (40.3) was obtained, is fulfilled only partially in the given case. This circumstance and the calculated approximation of the expressions of the weight coefficients led to the fact that an a priori estimation by this formula and an a posteriori estimation (from the results of adjustment) were somewhat different. However, the difference in the estimates comprises only $17 \%$ - a value which may not be considered high.

The second basic problem of the triangulation design, essentially the reverse of that considered - calculation of the optimum characteristics of the design according to the given accuracy is more complicated. Let us consider the more interesting version of this problem in the practical sense in the example of a space triangulation network, constructed from the chord directions.

1. The density and required accuracy of calculating the points, which are determined by the purpose of the given network, are assumed to be known. The mean square error in calculating the direction to the AES $m_{B}$ is also known. Since density may always be expressed by the average length of the sides between the ground points $D$ and the accuracy of the network may be characterized by the relative error in the common position of two adjacent points $\nu$, we will assume the values $D, \nu$, and $m_{\beta}$ to be known.

The required accuracy of calculating the chords, the number of synchronization planes, the maximum distances to the AES and the value of the semimajor axis of the orbit (in the first approximation - the radius of a circular orbit) should be found from these data. 
2. A second version of this problem is possible whose initial data will be $v, \mathrm{~m}_{B}$, and $\mathrm{H}$ - the satellite altitude, i.e., a rather frequent situation with a satellite already selected for observations, will be considered.

Let us calculate the main characteristics of a design using the first version. Let us first establish the relationship between the value $\nu$ and the error in the position of the point $\mathrm{M}_{j}$ in a space triangulation network, constructed by the method of chords.* The error in the common position of two adjacent points $\mathrm{m}_{j j}$ may be represented as

$$
m_{i i}^{3}=-t_{1}^{2}+t_{2}^{3}
$$

where $t_{j}$ are the projections of errors in the position of points onto the chord connecting these points. With uniform distribution of the position error vector with respect to the coordinate axes, we obtain

$$
t_{j}^{2}=\frac{1}{3} M_{j}^{2}
$$

Thus

$$
v^{2}=\frac{m_{i i}^{2}}{D^{2}}=\frac{1}{3} \cdot \frac{M_{i_{1}}^{2}+M_{i_{2}}^{2}}{D^{2}}
$$

Further assuming for a priori calculations $M_{j_{1}}=M_{j_{2}}=M_{j}$, we will have

$$
v=\sqrt{\frac{\overline{2}}{3}} \cdot \frac{M_{j}}{D}
$$

*Taking into account the equivalency of the various methods of space triangulation, the method of chords was selected only for computational convenience. 
On the other hand, the error in the position of the point is expressed approximately by Formula (40.3).

Let us assume that the average number of chords, convergent at a given point $N=5$. Then from Formulas (40.3) and (40.7), we obtain

$$
\frac{m_{B}^{n}}{\rho^{n}}=1,12 v
$$

Thus, the design will be accomplished with the given accuracy, if the errors in the direction of the chords are subject to Condition (40.8). Having substituted the expression for the error in the direction of the chord in angular measurements from Expressions (40.1) into (40.8), we obtain

$$
v \cong 1,25 \frac{m_{p}^{*} \rho}{D} \frac{}{\sqrt{\left(\sin ^{2} \lambda\right) \operatorname{cp}} \sqrt{(n-1)} \rho^{n}}
$$

The five independent parameters in Formula (40.9) make it possible to achieve the required value of $v$ by combining them in different ways. By definition, we are given the average length of chord $D$ and the error of the topocentric direction to the AES. At the present time, depending on the type of cameras and the errors in astrometric processing of photographs, the latter varies 1201 within the range of $0.7-2.0 "$. The values $\rho$ and $n$ may vary over a wide range, but the variation of their ratio is important. From Expression (40.9), we have

$$
\frac{\rho}{\sqrt{n-1}}=-\frac{v D \sqrt{\left(\sin ^{2} \lambda\right)_{c p}}}{1,25 m_{\beta}^{*}} \rho^{\prime \prime}
$$


The optimum value of both the geometric characteristics $(\rho, \lambda)$ and the quantitative characteristics $(n)$ for the given average distance between the points (D) and the accuracy of their mutual position $(v)$ is established on the basis of the variation of this ratio.

Since the time of conducting the observation session $T$ depends on the number of measurements $n$, required to achieve the given accuracy, it is proper to outline briefly the order of calculating this characteristic, proposed by Lambeck [58].

The total time $T$ is a function of the frequency of AES passage through the zone of observations $f$ and may be expressed as

$$
T=\frac{n}{f\left(1-p_{1}\right)}
$$

where $p_{1}$ is the probability of losses of possible observations due to unfavorable meteorological conditions, fallures of observation equipment, interruption of observations during preliminary processing, and non-simultaneity of observations.

The frequency of the passages every 24 hours may in turn be represented by the expression

$$
f=\frac{1440 p_{2} p_{3}}{\Delta t}
$$

where $\mathrm{p}_{2}$ is the probability of finding the AES in the zone of common visibility of the observation points; $p_{3}$ is the average probability of visibility of the AES; and $\Delta t$ is the time of finding the AES in the zone of common visibility (in minutes). 
In this case $p_{2}$ and $\Delta t$ depend on the area of the zone of common visibility $(S)$, related to the geometric characteristics of the network ( $\rho D$ ) and to the altitude of the AES ( $H$ ) by Expression $(37.10)$.

One version is possible of calculating the optimum characteristics of the design, with the total observation time $T$ being given. In this case, the order of calculations will be reversed and will include determining the maximum value of $\mathrm{n}$ as functions of the given geometric characteristics $D$ or $H$ and the optimum value of the zone of common visibility $S$, and then - calculating the expected accuracy of the results $(\nu)$ with consideration of the given measurement accuracy $\left(m_{\beta}\right)$. 


\section{LITERATURE}

1. Amelin, V. M. Methods of Using the Moon for Geodetic Purpọses. Byulleten' ITA, Vol. 6, No. I(84), 1958.

2. Amelin, V. M. The Possibility of Establishing the Relationship between Various Triangulation Systems by AES Observations. BSON, ISZ, No. 31, 1962.

3. Batrakov, Yu. V. On the Requirements for the Accuracy of Optical Observations of AES for Geodesy. BSON, ISZ, No. 55, 1969.

4. Bakulin, P. I. Fundamental'nyye katalogi zvezd (Fundamental Star Catalogs). GTI, Moscow-Leningrad, 1949.

5. Berrot, A. and W. Hoffmann. Space Geodesy. Foreign Languages Publishing House (IL), 1963.

6. Bugoslavskaya, N. Ya. Fotograficheskaya astrometriya (Photographic Astrometry). "Nauka" Press, MoscowLeningrad, 1947.

7. Boyko, Ye. G. Investigation of Certain Factors which Affect the Accuracy of Calculating Points. Izvestiya vuzov, Geodeziya i aerofotos"yemka, No. 4, 1969.

8. Boyko, Ye. G. Types of Conditions which Occur in Space Triangulation Networks. Geodeziya i kartografiya, No. $12,1969$.

9. Bursha, M. The Theory of Calculating the Non-Parallelism of the Minor Axis of the Reference Ellipsoid of the Polar Axis of the Earth's Inertia and of the Planes of the Initial Astronomic and Geodetic Meridians from AES observations. Studia geoph. et geod., No. 6, 1962.

10. Burxha, M. Osnovy kosmicheskoy geodezil (Fundamentals of Space Geodesy). "Nedra" Press, Moscow, 1971.

11. Veis, G: Geodezicheskoye ispol'zovaniye iskusstvennykh sputnikov zemli (Geodetic Use of Artificial Earth Satellites). "Nedra" Press, 1966. 
12. Ve1s, G. On the Optimum Use of Satellites for Geodesy. Geodeziya i kartografiya, No. 3, 1966.

13. Deych, A. N. On the Problem of the Reduction of Photographic Positions in an Arbitrary Optical Center. Astronomicheskiy zhurnal, Vol. 49, No. 5, 1965.

14. Dobachevska, V. and V. Baran. Adjustment of the Experimental Central European Space Triangulation Network and Analysis of the Results of Adjustment. Geodeziya 1 kartografiya, No. 9, 1967.

15. Zhongolovich,I. D. Earth Satellites and Geodesy. Astronomicheskiy zhurnal, Vol. 38, No. I, 1961.

16. Zhongolovich, I. D. Coordinate Systems Used in Investigations of the Motion of Artificial Earth Satellites. BSON ISZ, No. 31, 1962 .

17. Zhongolovich, I. D. Earth Satellites and Geodesy. Astro- 1203 nomicheskiy zhurnal, Vol. 16, No. I, 1964.

18. Zhongolovich, I. D. Design of a Single Worldwide Space Triangulation Network. Stud. geoph. et geod., No. 9, 1965.

19. Zhongolovich, I. D. Calculation of the Earth's Center of Mass with the Ald of Artificial Satellites. Nablyudeniya ISZ, No. 7, 1967 .

20. Zhongolovich, I. D. Design of the Arctic-Antarctic Geodetic Trajectory. BSON ISZ, No. 57, 1970.

21. Zagrebin, D. V. Vvedeniye $v$ astrometriyu (Introduction to Astrometry). "Nauka" Press, Moscow-Leningrad, 1966.

22. Zelinskiy, Ya. Solution of the Space Triangulation Problem Using the Known Orbital Elements of AES. Nablyudeniya ISZ, No. 3., 1964 .

23. Idel'son, N. I. Redustion Calculations in Astronomy. Astronomicheskiy yezhegodnik na 1941 (Astronomical Yearbook for 1941), Moscow-Leningrad, 1940.

24. Izotov, A. A. On the Theory of Calculating the Figures and Dimensions of the Earth from Observations of Artificial Satellites. Izvestiya vuzov, Geodeziya 1 aerofotos" yemka, No. 3, 1965. 
25. Kaula, V. M. Kosmicheskaya geodeziya (Space Geodesy). "Nedra" Press, Moscow, 1965.

26. Kaula, V. M. Sputnikovaya geodeziya (Satellite Geodesy). "Mir" Press, Moscow, 1970.

27. Kiselev, A. A., B. A. Firago and D. Ye Shchegolev. Instructions on Calculation of AES Coordinates from Photographs Obtained by NAFA-3c/25 Cameras. BSON ISZ, No. 3(13), 1960 .

28. Kosmicheskiye trayektornyye izmereniya (Space Trajectory Measurements). Eds., P. A. Agadzhanov, V. Ye. Dulevich, and A. A. Korostelev. Sovestkoye radio, Moscow, 1969.

29. Krasovskiy, F. N. and V. V. Danilov. Rukovodstvo po vysshey geodezil (Handbook on Higher Geodesy). GUGSK NKVD, Part 1, No. 1, Moscow, 1938.

30. Klenitskiy, B. M. Construction of Space Triangulation from the Directions and Lengths of Chords. BSON ISZ, No. 55, 1969.

31. Klenitskiy, B. M. and G. A. Ustinov. On the Optimum Shapes of Space Triangulation Figures. Geodeziya $i$ kartografiya, No. $1,1968$.

32. Klenitskiy, B. M. Adjustment of Three-Dimensional Space Triangulation in a System of Rectangular Geocentric Coordinates. Geodeziya 1 kartografiya, No. 5, 1964.

33. Klenitskiy, B. M. and G. A. Ustinov. Calculation of the Equatorial Topocentric Coordinates of AES. BSON ISZ, No. $39,1964$.

34. Klenitskiy, B. M. and G. A. Ustinov. On the Accuracy of Elementary Construction of Space Triangulation. Nablyudeniya ISZ, No. 3., 1964.

35. Linnik, Yu. V. Metod naimen'shikh kvadratov 1 osnovy matematiko-statisticheskoy teoril obrabotki nablyudeniy (The Least Squares Method and the Fundamentals of Mathematical-Statistical Theory of Processing Observations). Fizmatgiz, Moscow, 1958.

36. Masevich, A. G. and A. M. Lozinskiy. Photographic Observations of Artificial Earth Satelites. Nauchniyye informatsii Astrosoveta AN SSSR (Scientific Information of the Astronomical Council of the Academy of Sciences, USSR), Moscow, 1970. 
37. Muller, I. Vvedeniye v sputnikovuyu geodeziyu (Introduction to Satellite Geodesy). "Mir" Press, Moscow, 1967.

38. Mikhaylov, A. A. What Observations of the Moon Can Contribute to Geodesy? Geodeziya i kartografiya, No. 9, 1957.

39. Nefed'yev, A. A. Karty rel'yefa krayevoy zony Luny na obshchem nulevom urovne (Relief Maps of the Marginal Zone of the Moon at the Common Datum Level). AOE, Kazan', No. 30, 1958 .

40. Podobed, V. V. Fundamental'naya astrometriya (Fundamental Astrometry). Fizmatgiz, Moscow, 1962.

41. Popovich, K. Use of the Circle of Coincidence in Solution of Certain Problems of Space Geodesy. Nablyudeniya ISZ, No. $3,1964$.

42. Pranis-Pranevich, I. Yu. Rukovodstvo po uravnitel'nym vychisleniyam $v$ triangulyatsii (Handbook on Adjusting Calculations in Triangulation). Geodezizdat, Moscow, 1956.

43. Potter, H. I. On the Problem of Using Observations of the Moon for Geodetic Purposes. Astronomicheskiy zhurnal, Vol. 35, No. 4, 1958.

44. Provorov, K. L. O tochnost1 sploshnykh setey triangulyatsii (On the Accuracy of Continuous Triangulation Networks). Geodezizdat, Moscow, 1956.

45. Razumov, O. S. Error Distribution in an Elementary Space Triangulation System. Izvestiya vuzov, Geodeziya $i$ aerofotos"yemka, No. 6, 1967.

46. Standartnaya zemlya (The Standard Earth). Eds., K. Lunquist and G. Veis. "Mir" Press, Moscow, 1969.

47. Tuzov, G. I. Vydeleniye 1 obrabotka informatsii v dopplerovskikh sistemakh (Data Retrieval and Processing in Doppler Systems). Sovetskoye radio, Moscow, 1967.

48. Ustinov, G. A. Adjustment of Three-Dimensional Space Triangulation. Nablyudeniya ISZ, No. 2, 1963.

49. Ustinov, G. A. The Combined Method of Space Triangulation Adjustment. Geodeziya i kartografiya, No. 7, 1967. 
50. Ustinov, G. A. On the Most Advantageous Construction of the Elementary Figures of Space Triangulation. Geodeziya i kartografiya, No. 11, 1970.

51. Charnyy, V. I. On Isochronous Derivatives. Collection: Iskusstvennyye sputniki Zemli, No. 16, 1963.

52. Khristov, V. D. Rasshireniya uravnivaniya po sposobu naimen'skikh kvadratov (Expansion of Adjustment by the Least Squares Method). Sofia, 1956.

53. Shchegolev, D. Ye., A. G. Masevich and B. G. Afanas'yev. Simultaneous Observations of the Echo-l AES for Geodetic Purposes. Vestnik AN SSSR, No. 7, 1964.

54. Banchereau, A. Exploitation des mesures Doppler, faites sur DI-C - DI-D dans un but de géodésie semi-dynamique (Reduction of Doppler Measurements Performed on DI-C DI-D for Semi-Dynamic Geodesy). Paris, 1969.

55. Brosche, Nowacki and Strobel. Systematic Differences $\mathrm{FK}-4-\mathrm{GC}$ and $\mathrm{FK}-4-\mathrm{N}-30$. Karlsruhe, 1964.

56. Hirose, $\mathrm{H}$. Note on Simultaneous Observations of Artificial Satellites for Geodetic Purposes. Space Research II. Amsterdam, 1961.

57. Lambeck, K. Optimum Station-Satellite Configuration for Simultaneous Observations to Satellites. SAO Special Report, No. 231, 1966.

58. Lambeck, K. Scaling a Satellite Triangulation Network with Laser Range Measurements. Stud. geoph. et geod., Vol. 4, 1968 .

59. Lerch, C. G. Satellite Tracking with a Laser. SAO Speclal Report, No. 236, 1966 .

60. Lerch, C. G. and M. R. Pearlman. Laser Ranging to Satellites. COSPAR XIIth Plenary Meeting, Prague, May, 1969 .

61. Markowitz, w. Use in Geodesy of the Results of Lunar Observations and Eventual Satellites. Bull. géod., No. $49,1958$.

62. Newton, R. R. Caracteristiques du véhicule spatial GEOS A. Réseau Géodésique Européen par observations de satellites (Characteristics of the GEOS A Space Vehicle. European Geodesic Network Using Satellite Observations). Paris, 1965. 
63. O'Keefe, J.A. and J. P. Anderson. The Earth's Equatorial Radius and the Distance of the Moon. Bull. Géod., No. 29, 1953.

64. Ramsayer, N. Satellite Geodesy and Satellite Navigation. Zfv., No. 7, 1966.

65. Reduction of Satellite Photographic Plates. J. Kovalevsky and G. Veis, eds. COSPAR Transactions, No. 7,. Paris, 1970.

66. Rimer, K., K. Killian and P. Meiss. Beltrage zur Theorie der geometrische Netz in Raum (Contribution to the Theory of Spatial Geometric Networks). Munich, 1969.

67. Robbins. Time in Geodetic Astronomy. Surv. Rev., XIX, 1967, p. 143.

68. Schlesinger and Bennet. Trial of a Projection Method for Measuring Photographs. M. N., Vol. 93, 1933, p. 382.

69. Scott, F. R. Estimates of the Accuracy of Positions Taken from Photographic Star Catalogs. The Use of Artificial Satellites for Geodesy, Vol. 1, Amsterdam, 1963.

70. Väısälä and Oterma. Anwendung der astronomischen Trian gulationsmethode (Application of the Astronomical Triangulation Method). Helsinki, 1960.

71. Vonbun, F. O. Satellite Trajectory Determinations and Their Expected Errors. COSPAR XIIth Plenary Meeting. Prague, May, 1969.

72. Veis, G. On the Optimum Use of Satellites for Geodesy. Buil. géod., No. 74, 1964.

73. Veis, G. Geodetic Uses of Artificial Satellites. Smithsonian Contr. to Astrophys., Vol. 3, No. 9, 1960.

74. Veis, G. The Precision Optical Satellite Tracking Net of .SAO. The Use of Artificial Satellites for Geodesy, Vol. I, Amsterdam, 1963.

75. Whitford, Angular Diameters of Stars from Osculations by the Moon. Astr. J., Vol. 52, 1947. 